\title{
Interventions to increase attendance for diabetic retinopathy screening (Review)
}

Lawrenson JG, Graham-Rowe E, Lorencatto F, Burr J, Bunce C, Francis JJ, Aluko P, Rice S, Vale L, Peto T, Presseau J, Ivers N, Grimshaw JM

Lawrenson JG, Graham-Rowe E, Lorencatto F, Burr J, Bunce C, Francis JJ, Aluko P, Rice S, Vale L, Peto T, Presseau J, Ivers N, Grimshaw JM.

Interventions to increase attendance for diabetic retinopathy screening. Cochrane Database of Systematic Reviews 2018, Issue 1. Art. No.: CD012054.

DOI: 10.1002/14651858.CD012054.pub2.

www.cochranelibrary.com 
TABLE OF CONTENTS

HEADER 1

ABSTRACT

PLAIN LANGUAGE SUMMARY

SUMMARY OF FINDINGS

BACKGROUND

OBJECTIVES

METHODS

RESULTS

Figure 1.

Figure 2.

Figure 3.

Figure 4.

Figure 5.

Figure 6.

Figure 7.

Figure 8.

Figure 9.

Figure 10.

DISCUSSION

AUTHORS' CONCLUSIONS

ACKNOWLEDGEMENTS

REFERENCES

CHARACTERISTICS OF STUDIES

DATA AND ANALYSES

Analysis 1.1. Comparison 1 Any quality improvement intervention compared to usual care, Outcome 1 Proportion of participants attending screening.

Analysis 2.1. Comparison 2 Stepped quality improvement intervention compared to intervention alone, Outcome 1 Proportion

of participants attending screening.

ADDITIONAL TABLES

APPENDICES

CONTRIBUTIONS OF AUTHORS

DECLARATIONS OF INTEREST

SOURCES OF SUPPORT

DIFFERENCES BETWEEN PROTOCOL AND REVIEW

INDEX TERMS 
[Intervention Review]

\section{Interventions to increase attendance for diabetic retinopathy screening}

John G Lawrenson ${ }^{1}$, Ella Graham-Rowe ${ }^{2}$, Fabiana Lorencatto 2 , Jennifer Burr 3 , Catey Bunce ${ }^{4}$, Jillian J Francis ${ }^{2}$, Patricia Aluko ${ }^{5}$, Stephen Rice $^{6}$, Luke Vale ${ }^{6}$, Tunde Peto 7 , Justin Presseau ${ }^{8}$, Noah Ivers 9 , Jeremy M Grimshaw 8,10

${ }^{1}$ Centre for Applied Vision Research, School of Health Sciences, City University of London, London, UK. 2School of Health Sciences, Centre for Health Services Research, City University London, London, UK. ${ }^{3}$ School of Medicine, Medical and Biological Sciences Building, University of St Andrews, Fife, UK. ${ }^{4}$ Department of Primary Care \& Public Health Sciences, Kings College London, London, UK. ${ }^{5}$ National Institute for Health Research (NIHR) Innovation Observatory, Newcastle University, Newcastle upon Tyne, UK. ${ }^{6}$ Institute of Health \& Society, Newcastle University, Newcastle upon Tyne, UK. ${ }^{7}$ Centre for Public Health, Queen's University Belfast, Belfast, UK. ${ }^{8}$ Clinical Epidemiology Program, Ottawa Hospital Research Institute, Ottawa, Canada. ${ }^{9}$ Department of Family and Community Medicine, Women's College Hospital, Toronto, Canada. ${ }^{10}$ Department of Medicine, University of Ottawa, Ottawa, Canada

Contact address: John G Lawrenson, Centre for Applied Vision Research, School of Health Sciences, City University of London, Northampton Square, London, EC1V 0HB, UK. j.g.lawrenson@city.ac.uk.

Editorial group: Cochrane Eyes and Vision Group.

Publication status and date: New, published in Issue 1, 2018.

Citation: Lawrenson JG, Graham-Rowe E, Lorencatto F, Burr J, Bunce C, Francis JJ, Aluko P, Rice S, Vale L, Peto T, Presseau J, Ivers N, Grimshaw JM. Interventions to increase attendance for diabetic retinopathy screening. Cochrane Database of Systematic Reviews 2018, Issue 1. Art. No.: CD012054. DOI: 10.1002/14651858.CD012054.pub2.

Copyright $@ 2018$ The Cochrane Collaboration. Published by John Wiley \& Sons, Ltd.

\section{A B S T R A C T}

\section{Background}

Despite evidence supporting the effectiveness of diabetic retinopathy screening (DRS) in reducing the risk of sight loss, attendance for screening is consistently below recommended levels.

\section{Objectives}

The primary objective of the review was to assess the effectiveness of quality improvement (QI) interventions that seek to increase attendance for DRS in people with type 1 and type 2 diabetes.

Secondary objectives were:

To use validated taxonomies of QI intervention strategies and behaviour change techniques (BCTs) to code the description of interventions in the included studies and determine whether interventions that include particular QI strategies or component BCTs are more effective in increasing screening attendance;

To explore heterogeneity in effect size within and between studies to identify potential explanatory factors for variability in effect size; To explore differential effects in subgroups to provide information on how equity of screening attendance could be improved; To critically appraise and summarise current evidence on the resource use, costs and cost effectiveness.

\section{Search methods}

We searched the Cochrane Library, MEDLINE, Embase, PsycINFO, Web of Science, ProQuest Family Health, OpenGrey, the ISRCTN, ClinicalTrials.gov, and the WHO ICTRP to identify randomised controlled trials (RCTs) that were designed to improve attendance for DRS or were evaluating general quality improvement (QI) strategies for diabetes care and reported the effect of the intervention on DRS attendance. We searched the resources on 13 February 2017. We did not use any date or language restrictions in the searches.

\section{Selection criteria}

We included RCTs that compared any QI intervention to usual care or a more intensive (stepped) intervention versus a less intensive intervention. 


\section{Data collection and analysis}

We coded the QI strategy using a modification of the taxonomy developed by Cochrane Effective Practice and Organisation of Care (EPOC) and BCTs using the BCT Taxonomy version 1 (BCTTV1). We used Place of residence, Race/ethnicity/culture/language, Occupation, Gender/ sex, Religion, Education, Socioeconomic status, and Social capital (PROGRESS) elements to describe the characteristics of participants in the included studies that could have an impact on equity of access to health services.

Two review authors independently extracted data. One review author entered the data into Review Manager 5 and a second review author checked them. Two review authors independently assessed risks of bias in the included studies and extracted data. We rated certainty of evidence using GRADE.

\section{Main results}

We included 66 RCTs conducted predominantly (62\%) in the USA. Overall we judged the trials to be at low or unclear risk of bias. QI strategies were multifaceted and targeted patients, healthcare professionals or healthcare systems. Fifty-six studies (329,164 participants) compared intervention versus usual care (median duration of follow-up 12 months). Overall, DRS attendance increased by $12 \%$ (risk difference (RD) $0.12,95 \%$ confidence interval $(\mathrm{Cl}) 0.10$ to 0.14 ; low-certainty evidence) compared with usual care, with substantial heterogeneity in effect size. Both DRS-targeted (RD 0.17, 95\% Cl 0.11 to 0.22 ) and general QI interventions (RD $0.12,95 \% \mathrm{Cl} 0.09$ to 0.15 ) were effective, particularly where baseline DRS attendance was low. All BCT combinations were associated with significant improvements, particularly in those with poor attendance. We found higher effect estimates in subgroup analyses for the BCTs 'goal setting (outcome)' (RD 0.26, 95\% Cl 0.16 to 0.36 ) and 'feedback on outcomes of behaviour' ( $\mathrm{RD} 0.22,95 \% \mathrm{Cl} 0.15$ to 0.29 ) in interventions targeting patients, and 'restructuring the social environment' ( $\mathrm{RD} 0.19,95 \% \mathrm{Cl} 0.12$ to 0.26 ) and 'credible source' ( $\mathrm{RD} 0.16,95 \% \mathrm{Cl} 0.08$ to 0.24 ) in interventions targeting healthcare professionals.

Ten studies (23,715 participants) compared a more intensive (stepped) intervention versus a less intensive intervention. In these studies DRS attendance increased by $5 \%$ (RD $0.05,95 \% \mathrm{Cl} 0.02$ to 0.09 ; moderate-certainty evidence).

Fourteen studies reporting any QI intervention compared to usual care included economic outcomes. However, only five of these were full economic evaluations. Overall, we found that there is insufficient evidence to draw robust conclusions about the relative cost effectiveness of the interventions compared to each other or against usual care.

With the exception of gender and ethnicity, the characteristics of participants were poorly described in terms of PROGRESS elements. Seventeen studies (25.8\%) were conducted in disadvantaged populations. No studies were carried out in low-or middle-income countries.

\section{Authors' conclusions}

The results of this review provide evidence that QI interventions targeting patients, healthcare professionals or the healthcare system are associated with meaningful improvements in DRS attendance compared to usual care. There was no statistically significant difference between interventions specifically aimed at DRS and those which were part of a general QI strategy for improving diabetes care. This is a significant finding, due to the additional benefits of general QI interventions in terms of improving glycaemic control, vascular risk management and screening for other microvascular complications. It is likely that further (but smaller) improvements in DRS attendance can also be achieved by increasing the intensity of a particular QI component or adding further components.

\section{PLAIN LANGUAGE SUMMARY}

\section{Interventions to increase attendance for diabetic retinopathy screening}

\section{What is the aim of this review?}

The aim of this review was to find out if interventions used to improve attendance for diabetic retinopathy screening are effective.

\section{Key messages}

The results of this review found evidence that interventions that target patients, healthcare professionals or the healthcare system are likely to be effective for improving attendance for diabetic retinopathy screening compared to usual care. We found benefits for interventions that were specifically aimed at diabetic retinopathy screening, as well as those which were part of a general strategy to improve diabetes care. This is important, since more general strategies are associated with additional benefits, such as improving blood glucose control and increasing the detection of other diabetes-related complications.

\section{What was studied in the review?}

People with diabetes may lose vision as a result of the damaging effects of the disease on small blood vessels at the back of the eye (diabetic retinopathy). Screening for diabetic retinopathy to detect and treat early signs can prevent sight loss. However, screening attendance is variable and sight-threatening changes may not be detected in good time.

This review looked at a variety of interventions to improve diabetic retinopathy screening.

\section{What are the main results of the review?}


The Cochrane review authors found 66 relevant studies. Forty-one studies were from the USA, 14 from Europe, three from Canada, three from Australia and five from elsewhere. Fifty-six studies compared the intervention to improve screening attendance with usual care and 10 compared a more intensive to a less intensive intervention.

We found that interventions aimed at patients or healthcare professionals or both, or at the healthcare system were effective at improving screening attendance. Interventions aimed at improving the general quality of diabetes care worked as well as those specifically aimed at improving screening for retinopathy. On average, attendance increased by $12 \%$ compared with no intervention.

How up-to-date is this review?

The Cochrane review authors searched for studies that had been published up to 13 February 2017. 


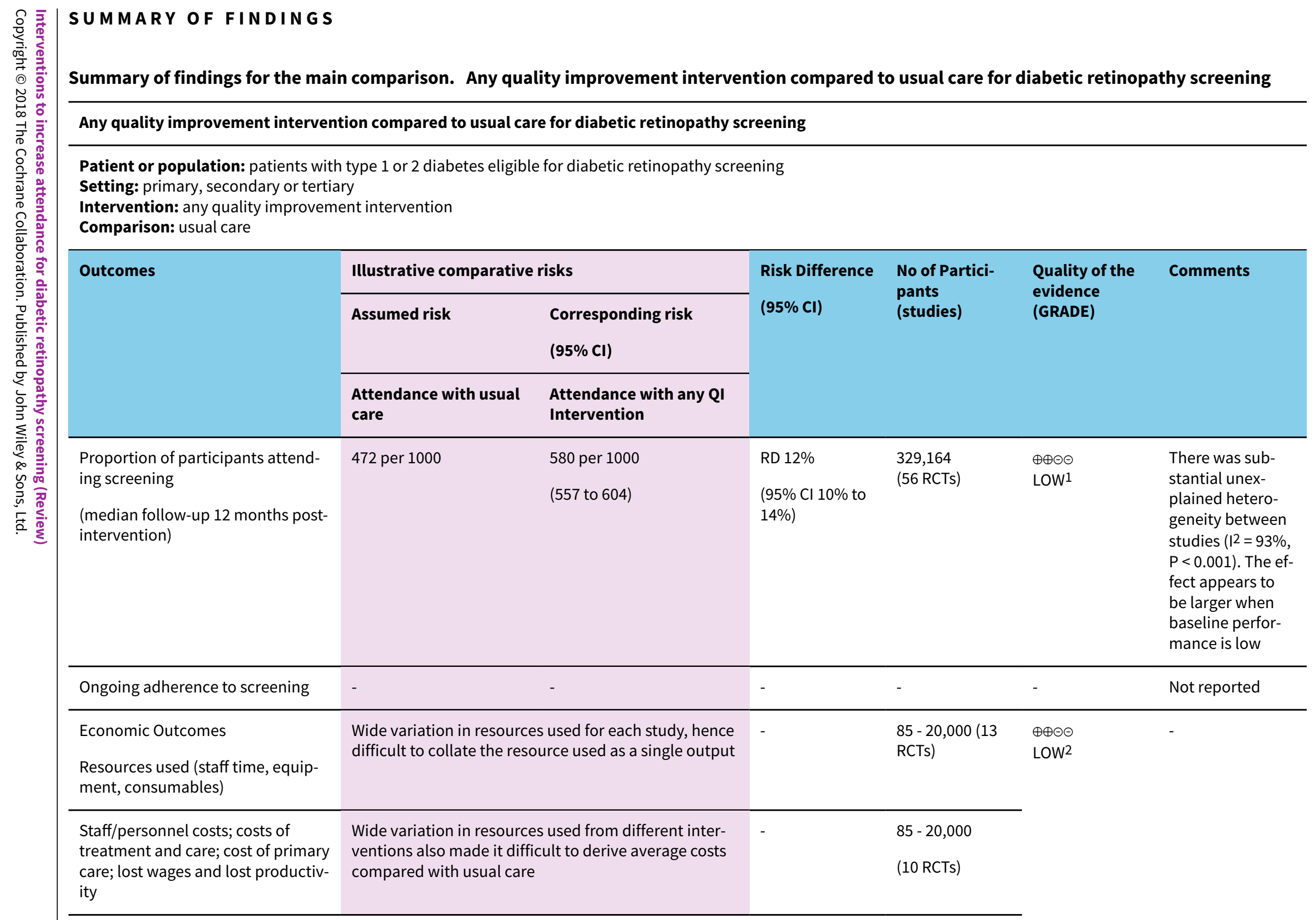


CI: Confidence interval; RD: Risk difference

GRADE Working Group grades of evidence

High-quality: Further research is very unlikely to change our confidence in the estimate of effect.

Moderate-quality: Further research is likely to have an important impact on our confidence in the estimate of effect and may change the estimate.

Low-quality: Further research is very likely to have an important impact on our confidence in the estimate of effect and is likely to change the estimate.

Very low-quality: We are very uncertain about the estimate.

${ }^{1}$ We downgraded the certainty of the evidence by two levels from high to low for inconsistency, due to wide variation in the effect estimates across studies that could not be explained.

2We downgraded the certainty of the evidence for the economic outcomes by two levels from high to low due to inconsistency across different elements of the economic outcomes (see Table 7).

\section{Summary of findings 2 . Stepped quality improvement intervention compared to intervention alone for diabetic retinopathy screening}

Stepped quality improvement intervention compared to intervention alone for diabetic retinopathy screening

Patient or population: patients with type 1 or 2 diabetes eligible for diabetic retinopathy screening

Setting: primary, secondary or tertiary

Intervention: stepped quality improvement intervention compared to intervention alone

Comparison: intervention alone

\begin{tabular}{|c|c|c|c|c|c|c|}
\hline \multirow[t]{3}{*}{ Outcomes } & \multicolumn{2}{|c|}{ Illustrative comparative risks } & \multirow{3}{*}{$\begin{array}{l}\text { Risk Difference } \\
(95 \% \mathrm{Cl})\end{array}$} & \multirow{3}{*}{$\begin{array}{l}\text { No of Partici- } \\
\text { pants } \\
\text { (studies) }\end{array}$} & \multirow{3}{*}{$\begin{array}{l}\text { Quality of the } \\
\text { evidence } \\
\text { (GRADE) }\end{array}$} & \multirow[t]{3}{*}{ Comments } \\
\hline & $\begin{array}{l}\text { Assumed risk } \\
(95 \% \mathrm{CI})\end{array}$ & $\begin{array}{l}\text { Corresponding risk } \\
(95 \% \mathrm{CI})\end{array}$ & & & & \\
\hline & $\begin{array}{l}\text { Attendance with } \\
\text { usual care }\end{array}$ & $\begin{array}{l}\text { Attendance with stepped } \\
\text { QI intervention }\end{array}$ & & & & \\
\hline $\begin{array}{l}\text { Proportion of participants attending } \\
\text { screening } \\
\text { (median follow-up } 12 \text { months post- } \\
\text { intervention) }\end{array}$ & 361 per 1000 & $\begin{array}{l}405 \text { per } 1000 \\
\text { (372 to } 437 \text { ) }\end{array}$ & $\begin{array}{l}\mathrm{RD} 5 \%(95 \% \mathrm{Cl} \\
2 \% \text { to } 9 \%)\end{array}$ & $\begin{array}{l}23,715 \\
\text { (10 RCTs) }\end{array}$ & $\begin{array}{l}\oplus \oplus \oplus \ominus \\
\text { MODERATE } 1\end{array}$ & $\begin{array}{l}\text { There was unex- } \\
\text { plained heterogene- } \\
\text { ity between studies } \\
(12=56 \%, P=0.02)\end{array}$ \\
\hline Ongoing adherence to screening & - & - & - & - & - & - \\
\hline
\end{tabular}




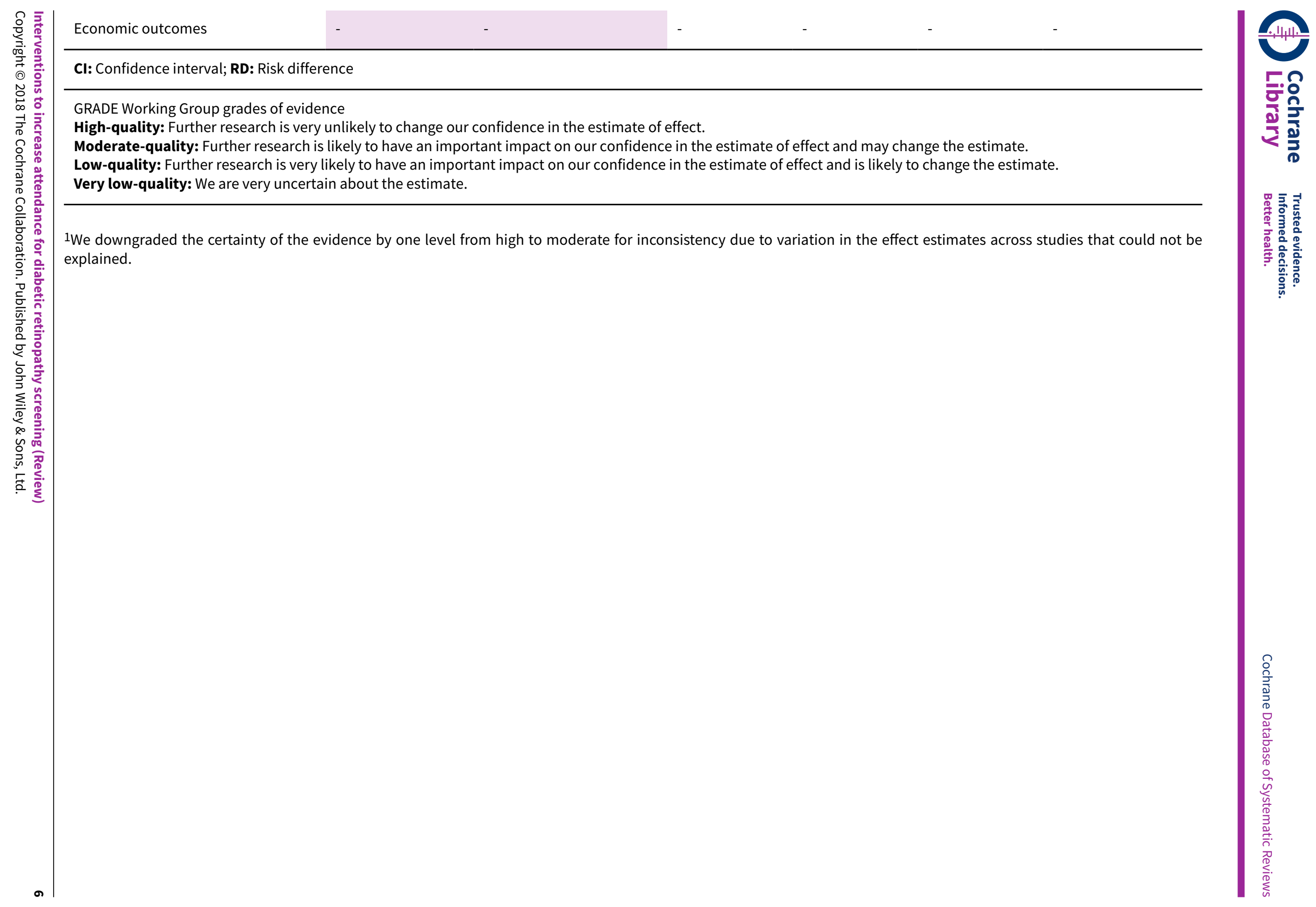




\section{B A C K G R O U N D}

\section{Description of the condition}

Diabetic retinopathy is the most common microvascular complication of diabetes mellitus and a leading cause of blindness amongst the working-age adult population in the Western world (Sivaprasad 2012). The duration of diabetes is the strongest predictor for the development and progression of retinopathy. Within 20 years of diagnosis, nearly all patients with type 1 diabetes and more than $60 \%$ of patients with type 2 have retinopathy (Fong 2004). A higher prevalence of diabetic retinopathy is found in people of South Asian, African and Latin American descent, compared to white populations (Sivaprasad 2012). Further risk factors for the development and progression of diabetic retinopathy include: poor glycaemic control, hypertension and hyperlipidaemia (Yau 2012). It has been estimated that globally approximately 93 million individuals may have some form of diabetic retinopathy, with 28 million suffering from the sightthreatening end points of the disease (Yau 2012). There is limited evidence on the economic burden of diabetic retinopathy. One recent estimate for healthcare costs in Sweden was EUR 106,000 per 100,000 population a year, based upon a prevalence of diabetes of $4.8 \%$ (95\% confidence interval 4.7 to 4.9 ) (Heintz 2010). These costs exclude cost impacts on those with diabetic retinopathy and their families.

Although effective treatments are available for sight-threatening diabetic retinopathy in the form of laser photocoagulation (Evans 2014) and more recently the use of anti-vascular endothelial growth factor inhibitors (Virgili 2014), the success of these interventions is dependent on early detection and timely referral for treatment. Diabetic retinopathy screening (DRS) fulfils the World Health Organization (WHO) criteria for a screening programme (Scanlon 2008): namely, diabetes-associated visual impairment is an important public health problem; potentially sight-threatening retinopathy has a recognisable latent stage; a universally accepted and effective treatment is available; and screening has been shown to be cost-effective in terms of sight years preserved compared with no screening (Jones 2010). Annual or biennial DRS is recommended in many countries using a variety of screening modalities, including: ophthalmoscopy performed by a number of healthcare professionals (including ophthalmologists, optometrists, diabetic physicians) or using standard retinal photography or digital fundus imaging (American Diabetes Association 2015; Kristinsson 1995; Scanlon 2008). Recently, mathematical algorithms have been developed that provide individualised risk assessment for diabetic retinopathy and optimisation of screening intervals based on type and duration of diabetes, $\mathrm{HbAlc}$, systolic blood pressure, gender and the presence and grade of retinopathy (Lund 2016).

Relatively few countries have introduced a national populationbased DRS programme, and in most parts of the world screening remains non-systematic.

The reference standard for the detection of diabetic retinopathy consists of seven standard 35-degree colour photographic fields as described by the Early Treatment Diabetic Retinopathy Study (EDTRS) research group (EDTRS 1991). However this technique is impractical for widespread retinopathy screening. Although ophthalmoscopy through dilated pupils has traditionally been the method of choice for opportunistic screening, the procedure varies in diagnostic accuracy depending on the particular technique used (direct or indirect ophthalmoscopy) or the experience of the healthcare professional performing the test (Hutchinson 2000). Recent developments in digital retinal photography have facilitated the rapid acquisition of high-quality fundus images that can be stored and subsequently graded. Digital imaging combined with trained graders has been shown to be an effective screening tool to identify sight-threatening retinopathy (Williams 2004), and is increasingly gaining acceptance for population screening (Kirkizlar 2013; Sharp 2003; Silva 2009; Taylor 2007).

Despite evidence supporting the effectiveness of DRS in reducing the risk of sight loss, screening coverage is consistently below recommended levels (Millett 2006; Paz 2006; Saadine 2008). The high rates of non-attendance have major financial consequences. For example, the North and East Devon Diabetic Retinal Screening Service in the UK invited 22,651 people to participate in retinal screening between April 2009 and March 2010. Of those invited, 2137 (9.4\%) failed to attend for their appointment after three reminders. With each appointment costing GBP 34 in 2009 and GBP 37 in 2010, the total cost of non-attendance was GBP 78,259 (2009/2010 GBP) (Waqar 2012). Several factors have been shown to affect access and attendance for DRS, including ethnicity, younger age (less than 40 years), a longer duration of diabetes, and living in areas of high social deprivation (Byun 2013; Gulliford 2010; Hwang 2015; Kliner 2012).

\section{Description of the intervention}

Several interventions specifically aimed at improving DRS, including those targeting patients, health professionals or the healthcare system, have been shown to be effective in improving attendance across a range of retinopathy screening models (Zhang 2007). Examples of patient-focused interventions include: (1) educational programmes to increase awareness of diabetic retinopathy and promote self-management, and (2) the use of prompts/reminders. Provider-focused interventions include: (1) clinician education, and (2) audit and performance feedback. System interventions include: (1) team changes; (2) establishing electronic registration and recall, and (3) the use of telemedicine.

In addition to strategies that specifically target DRS, general quality improvement (QI) implementation strategies for diabetes care may also be effective in improving screening coverage. A recent systematic review and meta-analysis of trials assessing a number of predefined QI strategies to improve diabetes care reported that these were associated with a significant increase in DRS compared to usual care (risk ratio $1.22,95 \%$ confidence interval 1.13 to 1.32 ) (Tricco 2012). However, this review did not include studies where interventions were solely targeted at patients, and the authors were unable to distinguish the effectiveness of individual QI components or identify potential effect modifiers. Furthermore, the review did not include an economic perspective.

\section{How the intervention might work}

Most studies assessing the effectiveness of interventions to improve diabetes care (including those delivered specifically to improve DRS) often involve multicomponent interventions that attempt to change the behaviour of healthcare professionals (e.g. advising patients to attend DRS) or patients (e.g. actually attending), or both. As there is no consistent association between the number of intervention components and their effectiveness (Grimshaw 2004), the 'ideal' number of components in such 
programmes is unknown. Furthermore, given the complexity of interventions tested to date, it is not always clear which specific components are the effective elements of these interventions (i.e. the 'active ingredients'). Hence, the content of complex behaviour change interventions has been referred to as a 'black box' (Grimshaw 2014). There is evidence that the more clearly the 'active' components of a complex intervention are described, the more readily the intervention may be delivered in an effective, consistent and cost-effective manner (Michie 2009). Therefore, identification of the effective interventions for increasing attendance for DRS first requires clarity about intervention content and the functional relationship between components of interventions and the intended outcome. Cochrane Effective Practice and Organisation of Care (EPOC) have developed a taxonomy that can be used to classify intervention content in systematic reviews (EPOC 2015). Although the EPOC taxonomy provides a common language and a useful summary description of the intervention, the taxonomy may not be sufficiently detailed to specify the components of the intervention clearly (Presseau 2015). A complementary approach is to provide a comprehensive categorisation of the ingredients of the intervention in terms of the behaviour change techniques (BCTs) used. BCTs are defined as the 'observable, replicable and irreducible components of an intervention that are designed to alter or redirect causal processes regulating behaviour' (Michie 2013). Recently, a reliable taxonomy of 93 BCTs has been published (co-developed by team member $\mathrm{JF}$ ) to provide a common, consistent terminology (BCT Taxonomy version 1 (BCTTV1)), by which the component BCTs in complex interventions may be identified and described. Examples of BCT labels in this taxonomy include: 'goal setting,' 'self monitoring,' 'providing feedback on behaviour' and 'problem solving'. Review team members (JP, NI and JG) have successfully demonstrated the feasibility of using the BCT taxonomy within trials of QI interventions for diabetes care (Presseau 2015).

\section{Why it is important to do this review}

Given the value of screening for reducing the risk of sight loss amongst people with diabetes, it is essential that attendance for DRS is maximised as far as available resources allow. Wide geographical variation in screening coverage has been reported, with associated inequalities in outcomes. Given the incremental costs (resource use) and benefits (effects) associated with interventions to improve attendance for DRS, it is important to consider whether such strategies are worthwhile.

By identifying the active components of interventions that increase attendance for screening, this review will contribute to the identification of implementation strategies for early detection of sight-threatening retinopathy. Furthermore, by exploring the differential effects of interventions in particular subgroups the results may provide clues to help to reduce inequalities in screening attendance and determine the impact of inequity on intervention effectiveness and efficiency. Although there have been a number of systematic reviews on interventions to optimise adult screening programmes (Everett 2011; Holden 2010), it is likely that this evidence is not directly transferable to DRS. Screening for diabetic retinopathy differs from other forms of screening in that the target group already has significant contact with the healthcare system due to their underlying diabetes, and screening has to be life-long (i.e. annual or biennial surveillance is necessary).

\section{O B JECT IVES}

The primary objective of the review was to assess the effectiveness of QI interventions that seek to increase attendance for DRS in people with type 1 and type 2 diabetes.

Secondary objectives:

- To use validated taxonomies of QI intervention strategies and behaviour change techniques (BCTs) to code the description of interventions in the included studies and determine whether interventions that include particular QI strategies or component BCTs are more effective in increasing screening attendance;

- To explore heterogeneity in effect size within and between studies to identify potential explanatory factors for variability in effect size;

- To explore differential effects in subgroups to provide information on how equity of screening attendance could be improved;

- To critically appraise and summarise current evidence on the resource use, costs and cost effectiveness.

\section{METHODS}

\section{Criteria for considering studies for this review \\ Types of studies}

We considered randomised controlled trials (RCTs), both individually randomised and cluster-RCTs, conducted in a primary or secondary care setting, that were either specifically designed to improve attendance for DRS or were evaluating general strategies to improve diabetes care. Most commonly, the latter group of studies referred to 'quality improvement targets' or 'diabetes processes of care measures' as primary or secondary outcomes. We only included these studies if they reported on the effect of the intervention on DRS attendance.

To investigate cost effectiveness we included full economic evaluations (cost-effectiveness analyses, cost-utility analyses and cost-benefit analyses), cost analyses and comparative resourceutilisation studies conducted alongside or as part of an included RCT.

\section{Types of participants}

We included people with type 1 and type 2 diabetes mellitus who were eligible for DRS.

\section{Types of interventions}

We included RCTs that used any planned strategy or combination of strategies to improve attendance for diabetic DRS targeted at individuals with diabetes (e.g. reminders, promotion of selfmanagement), healthcare professionals (e.g. education, audit and feedback) or the healthcare system (e.g. electronic registries, team changes). Interventions included those specifically targeting DRS, as well as those that were part of a general strategy to improve processes of diabetes care. Comparator interventions were as specified in the included studies. 


\section{Types of outcome measures}

\section{Primary outcomes}

The primary outcome was the difference in DRS attendance (one or more visits) within a two-year period following implementation of the intervention. This could be based on self-reports, medical insurance claims databases or health-record audits (hospital, primary care physician or screening administration system record).

\section{Secondary outcomes}

We considered the following secondary outcomes:

- Ongoing adherence to screening based on attendance for screening following the initial screening post-intervention.

- Economic outcomes:

a. Resources (staff time, equipment, consumables) required to deliver interventions to increase attendance for screening

b. Costs of staff used to provide interventions; costs of treatment and care; cost of primary care; lost wages and lost productivity (work output)

c. Cost effectiveness (incremental cost-effectiveness ratios (ICERs); incremental cost per quality-adjusted life year (QALY); incremental cost per disability-adjusted life year (DALY); incremental cost-benefit ratios; net benefits).

\section{Search methods for identification of studies}

\section{Electronic searches}

The Cochrane Eyes and Vision Information Specialist conducted systematic searches in the following databases for RCTs and controlled clinical trials. There were no language or publication year restrictions. The date of the search was 13 February 2017.

- Cochrane Central Register of Controlled Trials (CENTRAL; 2017, Issue 2) (which contains the Cochrane Eyes and Vision Trials Register) in the Cochrane Library (searched 13 February 2017) (Appendix 1);

- MEDLINE Ovid (1946 to 13 February 2017) (Appendix 2);

- Embase Ovid (1980 to 13 February 2017) (Appendix 3);

- PsycINFO (1967 to 13 February 2017) (Appendix 4);

- Web of Science Conference Proceedings Citation Index-Science (CPCI-S) and Emerging Sources Citation Index (ESCI) (1990 to 13 February 2017) (Appendix 5);

- ProQuest Family Health (1990 to 13 February 2017) (Appendix 6);

- OpenGrey (1980 to 13 February 2017) (Appendix 7);

- ISRCTN registry (www.isrctn.com/editAdvancedSearch; searched 13 February 2017) (Appendix 8);

- US National Institutes of Health Ongoing Trials Register ClinicalTrials.gov (www.clinicaltrials.gov; searched 13 February 2017) (Appendix 9);

- World Health Organization International Clinical Trials Registry Platform (www.who.int/ictrp; searched 13 February 2017) (Appendix 10).

\section{Searching other resources}

We searched the reference lists of included studies to identify additional relevant studies. In particular, we used the reference list of included and excluded studies of a 2012 systematic review by members of the current review team ( $\mathrm{NI}$ and JG) (Tricco
2012), which investigated the effectiveness of QI strategies on the management of diabetes. Tricco 2012 identified studies which have multiple interventions to improve the quality of care in diabetes. Some of the studies in this review included attendance for DRS as one of the outcomes being assessed. However, since the information on screening for diabetic retinopathy was not reported in the abstract or coded in the MeSH or thesaurus headings, the electronic search strategy used in the current review did not identify a number of these studies. In addition to searching the reference list of Tricco 2012, we also obtained additional studies reporting retinopathy outcomes from the review team currently updating that review. The protocol for the updated review has been republished (Ivers 2014), as whilst the scope of the review remains the same, the update proposes an exploration of heterogeneity using an innovative meta-analytical approach.

We also contacted experts in the field to request information on any ongoing or unpublished studies that would be relevant for this review.

\section{Data collection and analysis}

\section{Selection of studies}

Two review authors (JGL and JB) independently screened the titles and abstracts of studies identified by the electronic searches. We obtained full-text copies of possibly relevant studies, resolving any differences of opinion regarding inclusion/exclusion by discussion. We documented reasons for exclusion at this stage.

\section{Data extraction and management}

Two review authors (JGL and EGR), working independently, extracted data from the included studies using a modified version of the Cochrane Effective and Organisation of Care (EPOC) data collection form (EPOC 2017), which incorporates information on study design, type and duration of interventions, participants, setting, methods, outcomes, and results. We translated studies in languages other than English and similarly extracted data in duplicate. Where numerical data were presented only in figures and not available from authors, two review authors performed data extraction by using Plot Digitizer open-source software.

For the extraction of data on the sociodemographic characteristics of participants that are known to be important from an equity perspective, we used the Place, Race, Occupation, Gender, Religion, Education, Socioeconomic status, Social status (PROGRESS) framework (O'Neill 2014), and also recorded whether any interventions were aimed at disadvantaged or low- and middleincome country populations, using the World Bank Atlas method.

An economics review author (PA) identified and further assessed studies judged potentially to include economic data. Data from included economic evaluations were extracted by one review author (PA) and checked by a second. We adapted data collection from the format and guidelines used to produce the structured abstracts of full economic evaluations for inclusion in the NHS Economic Evaluation Database, and redesigned them to accommodate the specific data required for our review (CDC 2012). We classified economic evaluations based on their analytical framework and coded them appropriately. 


\section{Coding of intervention content}

We coded extracted intervention descriptions from all of the included studies using a validated taxonomy to characterise the constituent components of each intervention. Cochrane EPOC has developed a comprehensive taxonomy to classify intervention content in systematic reviews (EPOC 2015). We used a subset of the EPOC taxonomy that has been previously used by members of the review team in a review of the effectiveness of general QI implementation strategies for diabetes care (Tricco 2012). This adapted taxonomy incorporates 12 components targeting healthcare systems (case-management, team changes, electronic patient registry, facilitated relay of information to clinicians, continuous quality improvement), clinicians (audit and feedback, clinician education, clinician reminders, financial incentives) or patients (patient education, promotion of self-management and reminder systems). Two review authors (JGL and EGR) independently coded QI components as 'present' or 'absent' for both intervention and control arms, resolving discrepancies in coding by discussion.

To better characterise the detail of the intervention content, we also coded extracted intervention descriptions into component BCTs using the BCT taxonomy (Michie 2013), as a coding framework. Describing an intervention in terms of BCTs (i.e. 'active ingredients') provides a useful level of detail for synthesis and comparison (Presseau 2015). We coded BCTs for each intended recipient as 'present' or 'absent' separately for patient and healthcare professional recipients. We coded each intervention separately, including control arms. We coded system-level interventions as targeting either healthcare provider or patient behaviour, or both, unless an alternative intervention recipient and their behaviour was reported (e.g. administrative staff sending reminder letters) (see Table 1).There is substantial evidence that the content of complex behaviour change interventions is often poorly described in published reports, rendering it more difficult to clearly specify the content of interventions on this basis alone and increasing the risk of misclassification (Lorencatto 2013). We therefore contacted all authors of included studies to ask for further information on the content of the intervention (e.g. a trial protocol, letters sent to patients, written or audio-visual materials) to clarify the BCT coding. We coded these materials using the BCT taxonomy in the same manner as for the corresponding published reports.Two review authors (EGR and FL) independently conducted BCT coding, resolving discrepancies by discussion and if necessary by the involvement of a third review author (JF).

\section{Coding of resource requirement needed to deliver interventions}

We developed an ordered ranking scale to quantify the level of resource needed to deliver each intervention, based on the description of the intervention components in each included study. To determine the feasibility of this approach, we initially piloted the scale on a sample of 10 included studies, using two members of the review team. We graded each intervention initially between one (least resource-intensive) and five (most resource-intensive), or zero (unable to determine), together with a record as to how the review author graded each study.

We incorporated the following resource components into the algorithm:

- Face-to-face minutes

- Phone calls

- Patient home visits

- Printed materials/software

- Training

The resource categories and levels with their corresponding weights were as follows:

\begin{tabular}{lllll}
\hline $\begin{array}{l}\text { Face-to-face or care planning min- } \\
\text { utes/patient/6 months }\end{array}$ & $\begin{array}{l}\text { Phone calls to } \\
\text { patients }\end{array}$ & $\begin{array}{l}\text { Additional out- } \\
\text { reach visits to pa- } \\
\text { tients (travel time) }\end{array}$ & $\begin{array}{l}\text { Use of materials/ let- } \\
\text { ters/software }\end{array}$ & $\begin{array}{l}\text { Training of health pro- } \\
\text { fessionals other than } \\
\text { reading material }\end{array}$ \\
\hline None (0) & No (0) & No (0) & None (0) & None (0) \\
\hline Low $\mathbf{1}-\mathbf{4 0}$ mins (1) & Yes (1) & Yes (2) & Printed materials (1) & Low (1) \\
\hline Moderate $\mathbf{4 0 - 1 0 0 ( 2 )}$ & - & - & Software (2) & High (2)
\end{tabular}

High $>100$ (3)

We defined a priori a criterion of success of the ranking scale as review author scores from nine out of 10 studies being within one grade of each other, following discussion. This criterion was achieved and we used the notes about how we graded each study to generate a reproducible description of the resource input associated with each grade on the ranking scale. We then used the resource components and their intensity levels to extract resource use required to deliver the interventions in all included studies. Two review authors (JGL and EGR) did this independently.

\section{Assessment of risk of bias in included studies}

Two review authors (JGL and EGR) independently assessed study quality using the Cochrane EPOC 'Risk of bias' tool (EPOC 2012). We based the choice of the EPOC 'Risk of bias' tool on the expectation that the included studies would be similar to those included in EPOC reviews, e.g. a large number of cluster trials, complex interventions and routine data used to assess outcomes.

The EPOC criteria for assessing risk of bias uses nine standard criteria: 
- Was the allocation sequence adequately generated?

- Was the allocation adequately concealed?

- Were baseline outcome measurements similar?

- Were baseline characteristics similar?

- Were incomplete outcome data adequately addressed?

- Was knowledge of the allocated interventions adequately prevented during the study?

- Was the study adequately protected against contamination?

- Was the study free from selective outcome reporting?

- Was the study free from other risks of bias?

For cluster-RCTs, we considered particular biases, including: (i) recruitment bias; (ii) baseline imbalance; (iii) loss of clusters, and (iv) incorrect analysis; as described in Chapter 16 of the Cochrane Handbook for Systematic Reviews of Interventions (Higgins 2011). For each domain, two review authors performed the 'Risk of bias' assessment independently and assigned a judgement of 'low risk' 'high risk' or 'unclear risk' of bias. The review authors resolved any discrepancies between them by discussion.

The reliability of data outputs from any full economic evaluation are in part predicated on the reliability of the data for the estimates of the relative treatment effects (for benefits or harms) of the alternative courses of action (i.e. intervention(s) and comparator(s)) under investigation). As the identified economic studies were a subset of the studies included in the review, the risk of bias was already assessed. However, assessment of the overall methodological quality of the economic component was still required and was carried out by one review author (PA) using the Consolidated Health Economic Evaluation Reporting Standard (CHEERS) statement, together with the Consensus on Health Economic Criteria (CHEC) (Evers 2005; Husereau 2013). In assessing the methodological quality of economic evaluations, the main objective is to assess the applicability of the scope of the analysis in terms of costs and outcomes. This helps to highlight the applicability and relevance of each economic evaluation.

\section{Measures of treatment effect}

Attendance at screening post-intervention is a dichotomous outcome and we have reported the intervention effect as the risk difference (RD), i.e. the actual difference in the observed events between experimental and control interventions. Our choice of RD was based on the fact that relative effect sizes (e.g. risk ratios) are highly dependent on the baseline/control compliance, i.e. a similar risk ratio if screening attendance increase from $10 \%$ to $20 \%$ or from $50 \%$ to $100 \%$. During the development of the protocol for the review, we received advice from the Cochrane EPOC group who have found that RDs are much more interpretable, and it is also possible to explore whether baseline compliance is an effect modifier.

\section{Unit of analysis issues}

For individual randomised trials the unit of analysis was the individual participant. For cluster-RCTs, we analysed data after adjustment for clustering. In case of cluster-RCTs, where outcomes were presented at patient level, we used an established method to adjust for clustering (Higgins 2011).This involved dividing the original sample size by the design effect, which was calculated from the average cluster size and the intra-cluster correlation coefficient
(ICC). Where the ICC was not reported, we imputed the most commonly-reported value from studies where it was reported.

\section{Dealing with missing data}

We contacted authors of included studies if important data were not available. Where we were not able to obtain these data, we reported the available results and did not impute missing data.

\section{Assessment of heterogeneity}

We assessed heterogeneity between studies by visual inspection of forest plots and by formal statistical tests of heterogeneity (Chi2 test and the $1^{2}$ statistic), and explored the possible reasons for heterogeneity using subgroup and random-effects meta-regression analyses.

\section{Assessment of reporting biases}

We explored publication bias using a funnel plot for the main comparison of any intervention versus usual care.

\section{Data synthesis}

We conducted meta-analyses in Review Manager 5 (Review Manager 2014), using a random-effects model to estimate the pooled RD across studies. We included data from RCTs randomised by individual and from cluster-adjusted RCTs in the same metaanalysis. In the case of multiple intervention groups, we combined groups to create a single pair-wise comparison as recommended in the Cochrane Handbook for Systematic Reviews of Interventions (Higgins 2011).

A summary of the results of included economic evaluations is available as an additional table (Table 2 ) and is supplemented by a narrative description in the Results and Discussion sections. Costs for each study were adjusted to 2016 British pound value (GBP) using a web-based conversion tool based on implicit price deflators for gross domestic product (GDP, a measure of the wealth of a country) and GDP Purchasing Power Parities. Table 2 presents the original currency and price year used in each included study. Users of this review who might want to adjust costs to another currency and price year suitable for their needs should use costs for each study presented in Table 2 and not the adjusted costs presented in the main text of the review.

\section{Subgroup analysis and investigation of heterogeneity}

We planned to perform the following prespecified subgroup analyses to investigate whether the presence or absence of a particular covariant explained the variability in effect size:

\section{- Ql intervention components/BCTs}

- Resource requirements to deliver the intervention

- Population subgroups: type 1, type 2 diabetes mellitus, participant characteristics across PROGRESS categories

In our analyses, we assessed QI components (coded using the modified EPOC taxonomy) and BCTs of each intervention separately. Where a study used multiple QI components or BCTs or both, we applied the same effect size to each component for the analysis. We compared effect estimates for subsets of studies that used a particular QI component/BCT or resource intensity and calculated a pooled effect size. We included BCTs/QI components 
in the analysis when 10 more studies were available for each BCT/ QI component.

We further investigated associations between DRS attendance and effect size by meta-regression for a number of covariates, including: type of study design (individual/cluster-RCT), baseline DRS attendance and QI component/BCT used in the intervention. For meta-regression we used a prespecified random-effects model and compared the risk difference of studies containing a particular explanatory variable to studies in which the variable was absent. For metaregression we followed the guidance in the Cochrane Handbook for Systematic Reviews of Interventions (Higgins 2011), and only included covariates for which 10 or more studies were available.

We conducted subgroup analyses and meta-regression using Stata 14 , deploying the metan and metareg commands.

\section{Sensitivity analysis}

We performed a sensitivity analysis to determine the impact on the pooled effect estimate of imputing the lower and upper range values for the ICC.

\section{'Summary of findings' Tables}

We prepared 'Summary of findings' tables for the main comparisons (1. effect of any QI intervention versus usual care on
DRS attendance and 2. effect of a more intensive (stepped) invertion versus a less intensive intervention). We assessed certainty of evidence (GRADE) for each outcome using customised software (GRADEpro GTD). One author (JGL) did the initial assessment which was then checked by other review authors. We considered risk of bias, inconsistency, indirectness, imprecision and publication bias when judging the certainty of the evidence.

\section{RES U L T S}

\section{Description of studies}

\section{Results of the search}

The electronic searches yielded 9030 records (Figure 1). The Cochrane Information Specialist removed 1786 duplicate records and we screened the remaining 7244 records plus 33 records identified from additional sources (Tricco 2012). We rejected 7152 records after reading the abstracts and obtained full-text reports of 125 references for further assessment. We identified 81 reports of 66 studies that met the inclusion criteria (see Characteristics of included studies) and excluded 34 reports of 34 studies (see Characteristics of excluded studies). We also identified nine reports of eight ongoing trials (see Characteristics of ongoing studies), and will assess these when results become available. 
Figure 1. Study flow diagram.

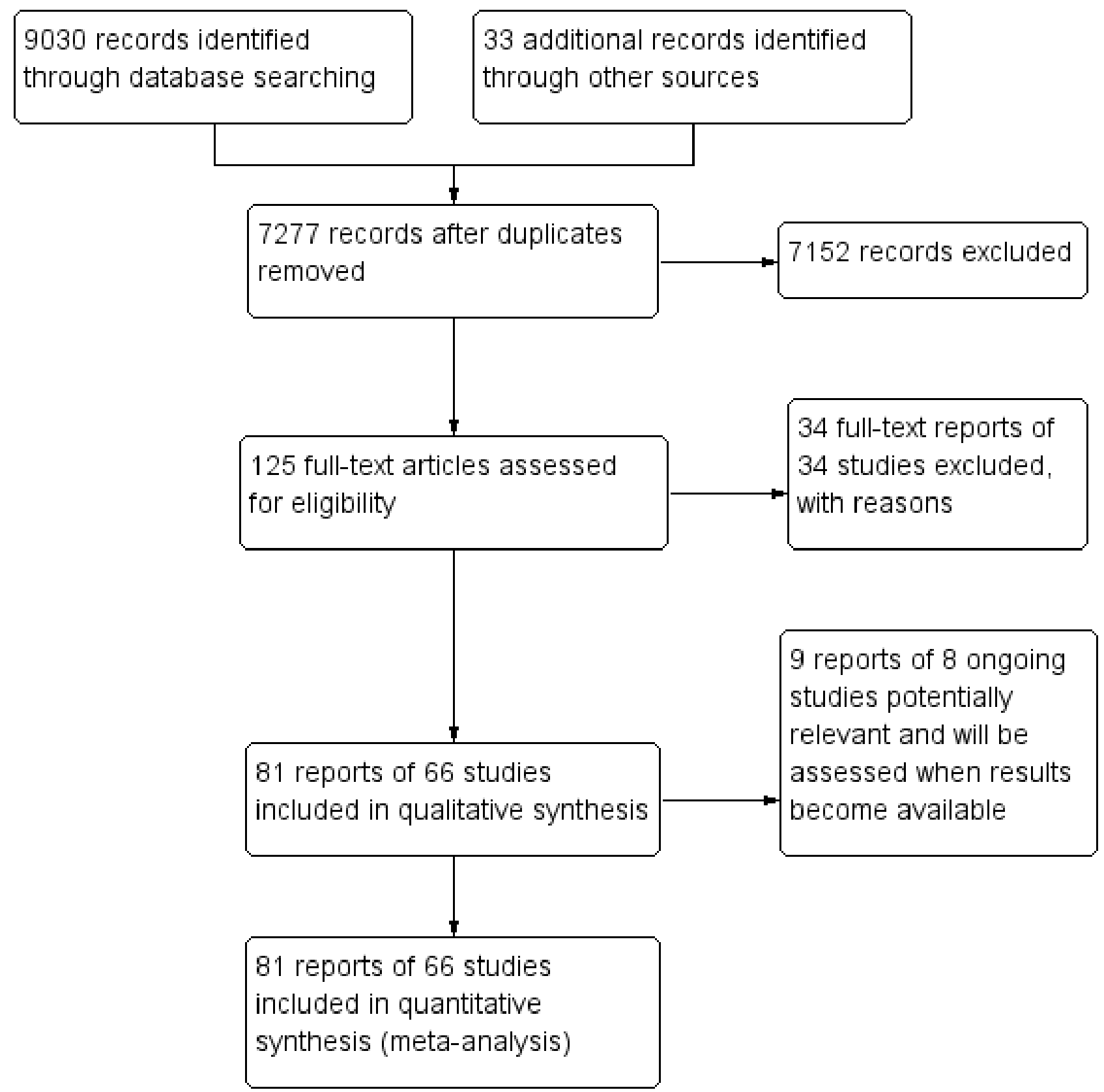

\section{Included studies}

The included studies were conducted between 1988 and 2013. Thirty-five studies (53\%) were parallel-group patient RCTs enrolling 237,025 patients, and $31(47 \%)$ were cluster-RCTs in which the healthcare professional or the healthcare setting was the unit of randomisation. These included 6126 clusters (range 6 to 4125). Fifty-nine studies (89.4\%) had two arms, six studies (9.1\%) had three arms and one study (1.5\%) had more than three arms. For further details see Characteristics of included studies.

\section{Types of participants}

Participant characteristics are reported in Table 3. Most of the studies $(57.6 \%)$ recruited participants with type 2 diabetes, $15.2 \%$ of studies included those with either type 1 or type 2 diabetes, and in $12.1 \%$ of studies the type of diabetes was not reported.

We used PROGRESS elements to describe the characteristics of participants in the included studies that could have an impact on equity of access to health services. With the exception of gender (reported in $93.9 \%$ of studies) and ethnicity (reported in $56.1 \%$ of studies), the characteristics of participants were poorly described, and the relative effectiveness of the interventions for subgroups in terms of PROGRESS elements was never reported. Seventeen studies $(25.8 \%)$ were conducted in disadvantaged populations and none were carried out in low- or middle-income countries. 


\section{Types of setting}

Details of study location and setting are given in Table 3. Most of the studies (62.1\%) were conducted in the USA, $21.2 \%$ in Europe and $16.7 \%$ elsewhere. The setting was primary care in $77.7 \%$, secondary care in $10.6 \%$ and unclear in $12.1 \%$.

Intervention content in terms of $\mathrm{QI}$ components (coded using the modified EPOC taxonomy)

Interventions were either specifically targeted at improving attendance for DRS ( $\mathrm{N}=16)$ or were part of a general QI intervention to improve diabetes care $(\mathrm{N}=50)$. For studies comparing any intervention to usual care, most studies provided no description of usual care, which precluded coding of the comparator arm.

All 12 QI intervention components, as defined by the modified EPOC taxonomy, were used in at least one study (Figure 2). Generally, interventions were multifaceted, with several QI components per intervention arm (median 3, range 1 - 7). For interventions specifically targeting DRS attendance, the most commonly used QI components were 'Patient reminders (56\% of studies)' and 'Patient education (75\%) (Figure 3). For general QI interventions, a greater number and range of strategies were used, including: 'Patient education' ( $48 \%$ of studies), 'Promotion of self-management' (40\%), 'Case management' (40\%), 'Clinician education' (38\%) and 'Team changes' (36\%).

Figure 2. Quality improvement components used in intervention arm of included studies. (DRS=diabetic retinopathy screening, $G Q I=$ general quality improvement).

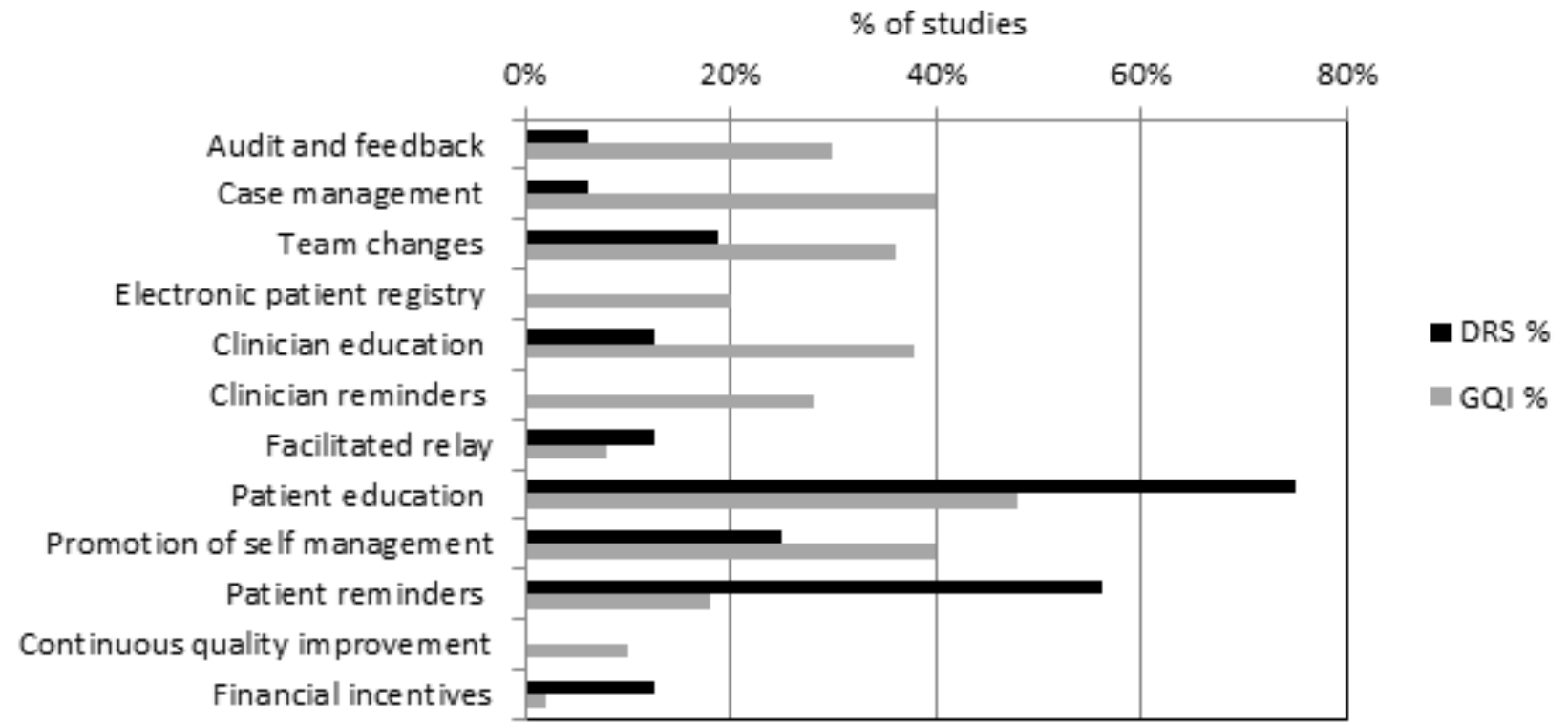


Figure 3. Risk of bias summary: review authors' judgements about each risk of bias item for each included study.

\begin{tabular}{|c|c|c|c|c|c|c|c|c|c|}
\hline & 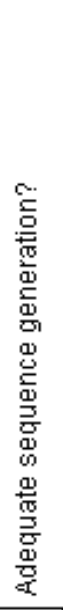 & 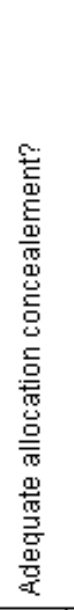 & 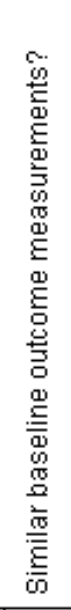 & 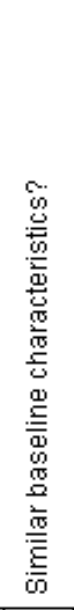 & 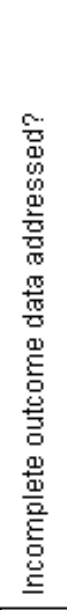 & 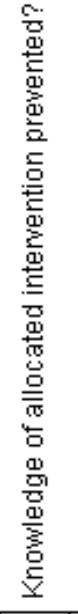 & 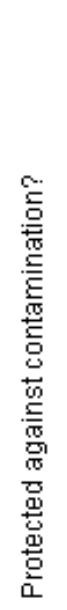 & 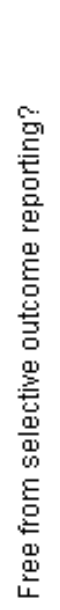 & 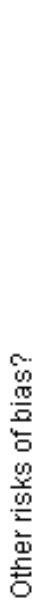 \\
\hline Adair 2013 & $\odot$ & $\odot$ & $\oplus$ & $\odot$ & $\odot$ & $\odot$ & $\odot$ & + & + \\
\hline Anderson 2003 & $?$ & $?$ & $\odot$ & $\odot$ & + & $?$ & + & $?$ & + \\
\hline Barcelo 2010 & $?$ & $\odot$ & + & $\odot$ & $?$ & $?$ & $\Theta$ & $?$ & + \\
\hline Basch 1999 & + & $?$ & $\odot$ & $\odot$ & $?$ & + & + & $?$ & + \\
\hline Bush 2014 & $?$ & $\odot$ & $\odot$ & $?$ & 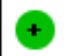 & + & + & $?$ & + \\
\hline Choe 2005 & + & $?$ & $?$ & $\odot$ & + & + & $?$ & $?$ & + \\
\hline Clancy 2007 & $\odot$ & $?$ & $?$ & $\odot$ & $\odot$ & + & $\odot$ & $?$ & + \\
\hline Conlin 2006 & + & $?$ & $?$ & $?$ & $\odot$ & $?$ & + & ? & + \\
\hline Davis 2003 & $?$ & $?$ & $?$ & $?$ & $?$ & $?$ & + & $?$ & $?$ \\
\hline Davis 2010 & $?$ & $?$ & $\odot$ & $\odot$ & + & $?$ & + & + & + \\
\hline Dickinson 2014 & $?$ & + & $\odot$ & $?$ & $?$ & $?$ & + & + & $?$ \\
\hline Dijkstra 2005 & $?$ & + & + & + & $\Theta$ & ? & + & ? & + \\
\hline Dijkstra 2008 & $?$ & $\odot$ & $\odot$ & $?$ & $\odot$ & $?$ & + & $?$ & $?$ \\
\hline Eccles 2007 & + & + & $\odot$ & $\odot$ & + & $\odot$ & + & + & + \\
\hline Ellish 2011 & $?$ & $?$ & $?$ & $\odot$ & $\odot$ & ? & + & $?$ & + \\
\hline Franco 2007 & ? & $\odot$ & + & + & $\theta$ & $\theta$ & + & ? & + \\
\hline Frei 2014 & + & + & $\odot$ & $\odot$ & $\odot$ & $?$ & + & $\oplus$ & + \\
\hline Frijling 2002 & $\odot$ & $\odot$ & $\odot$ & + & $\odot$ & + & + & $?$ & + \\
\hline Gabbay 2006 & - & $?$ & $?$ & + & $?$ & ? & + & $?$ & + \\
\hline Gabbay 2013 & $?$ & $?$ & $?$ & $\odot$ & $\Theta$ & $?$ & + & $\odot$ & \\
\hline Glasgow 2005 & $?$ & $\odot$ & $\odot$ & + & $?$ & $?$ & + & $?$ & + \\
\hline Guldberg 2011 & + & + & $?$ & + & + & + & + & $?$ & \\
\hline
\end{tabular}


Figure 3. (Continued)

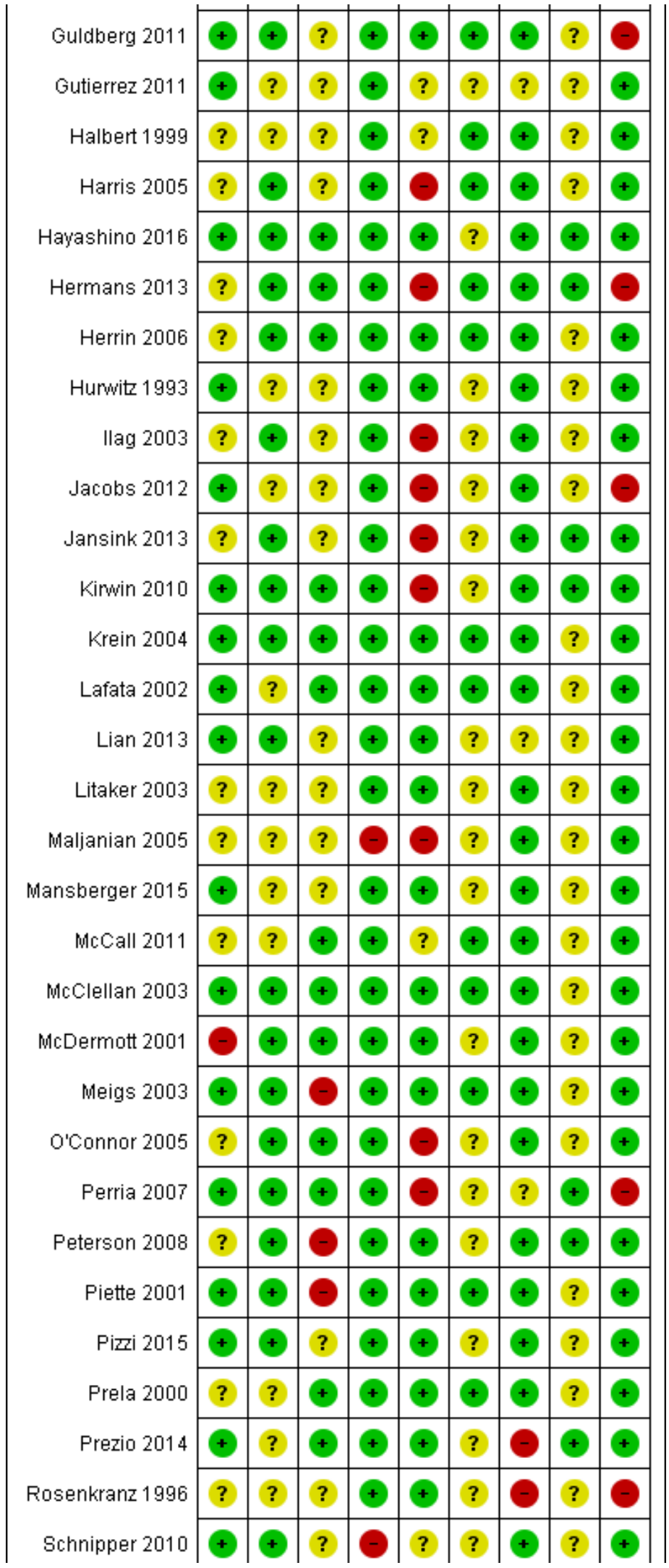


Figure 3. (Continued)

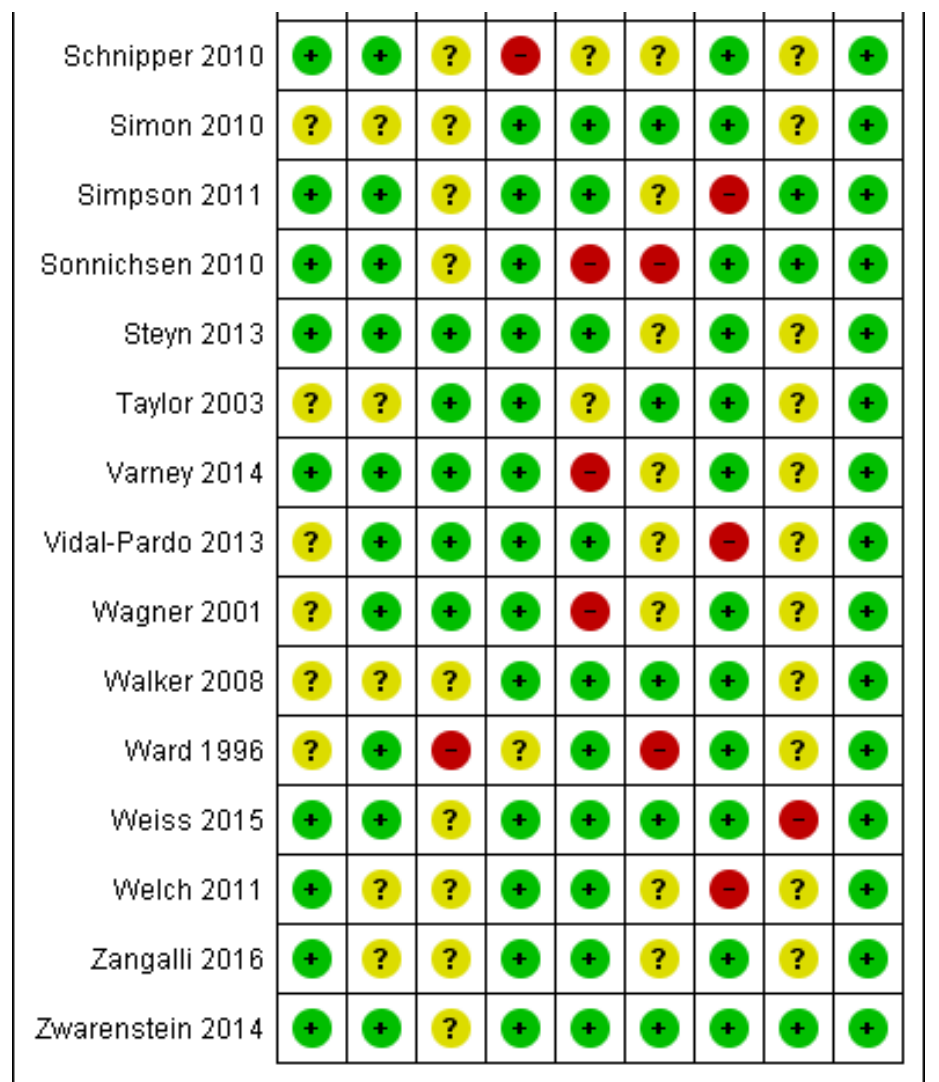

\section{Intervention content in terms of BCTs (coded using the BCT taxonomy)}

Overall, 39 out of the possible 93 BCTs (42\%) were identified as targeting change in patient or healthcare professional behaviour in at least one trial. Interventions specifically targeting DRS primarily used techniques aimed at patients, particularly 'Instruction on how to perform the behaviour' (75\% of studies), 'Prompts/ cues' (69\%) and 'Information about consequences' (56\%) (Figure 4). Relatively few of these studies used BCTs that were aimed at healthcare professionals (Figure 5). By contrast, these healthcare professional-directed strategies were more widely used in general QI interventions, in particular: 'Instruction on how to perform the behaviour' (66\%), 'Restructuring the social environment' (52\%) and 'Feedback on outcomes of behaviour/Biofeedback' (36\%). Table 1 provides illustrative quotations for each BCT. 
Figure 4. Behaviour change techniques (BCTs) targeting patients used in intervention arm. of included studies (DRS=diabetic retinopathy screening, $G Q I=$ general quality improvement).

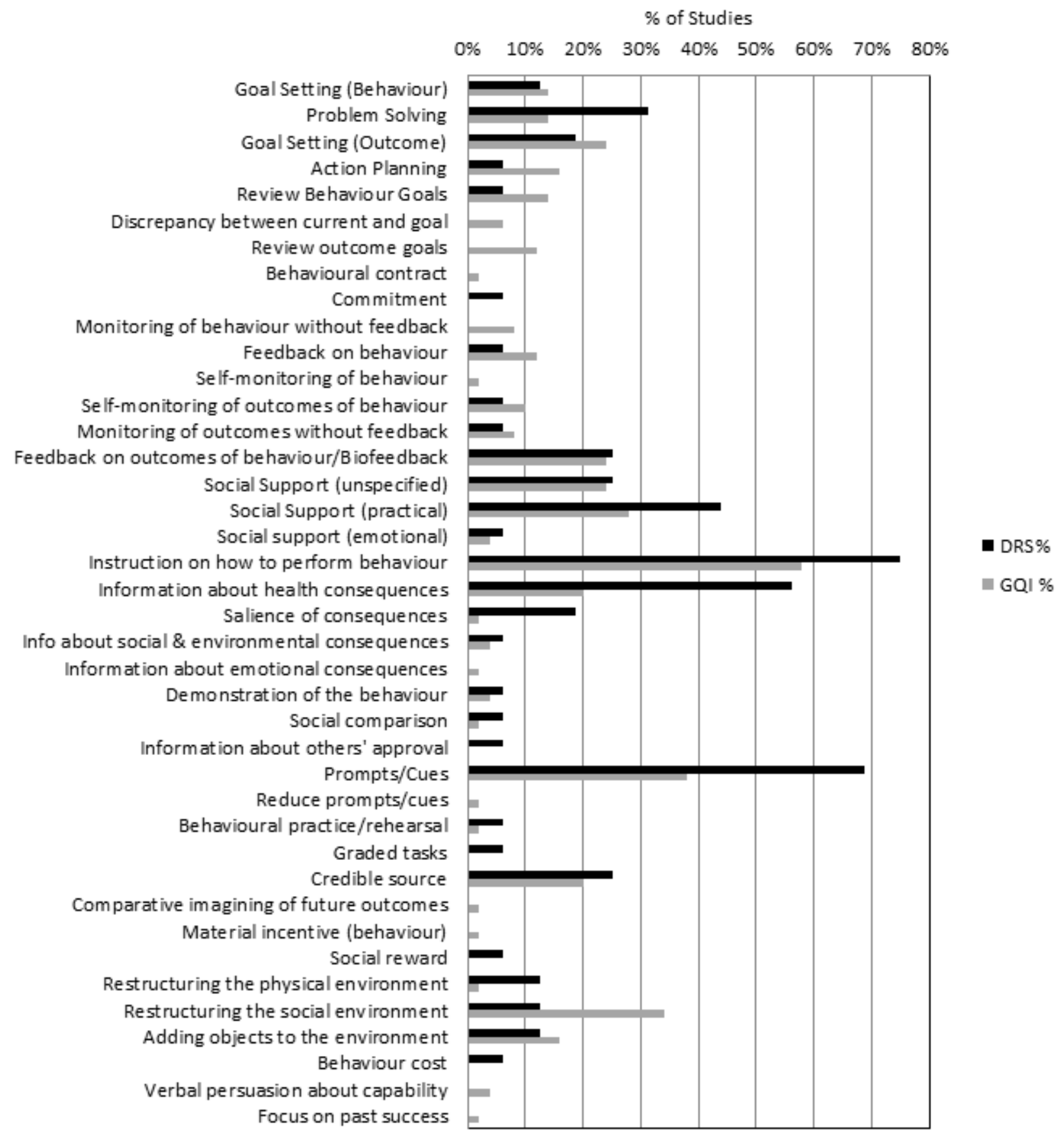


Figure 5. Behaviour change techniques (BCTs) targeting healthcare professionals used in intervention arm of included studies (DRS=diabetic retinopathy screening, $G Q I=$ general quality improvement).

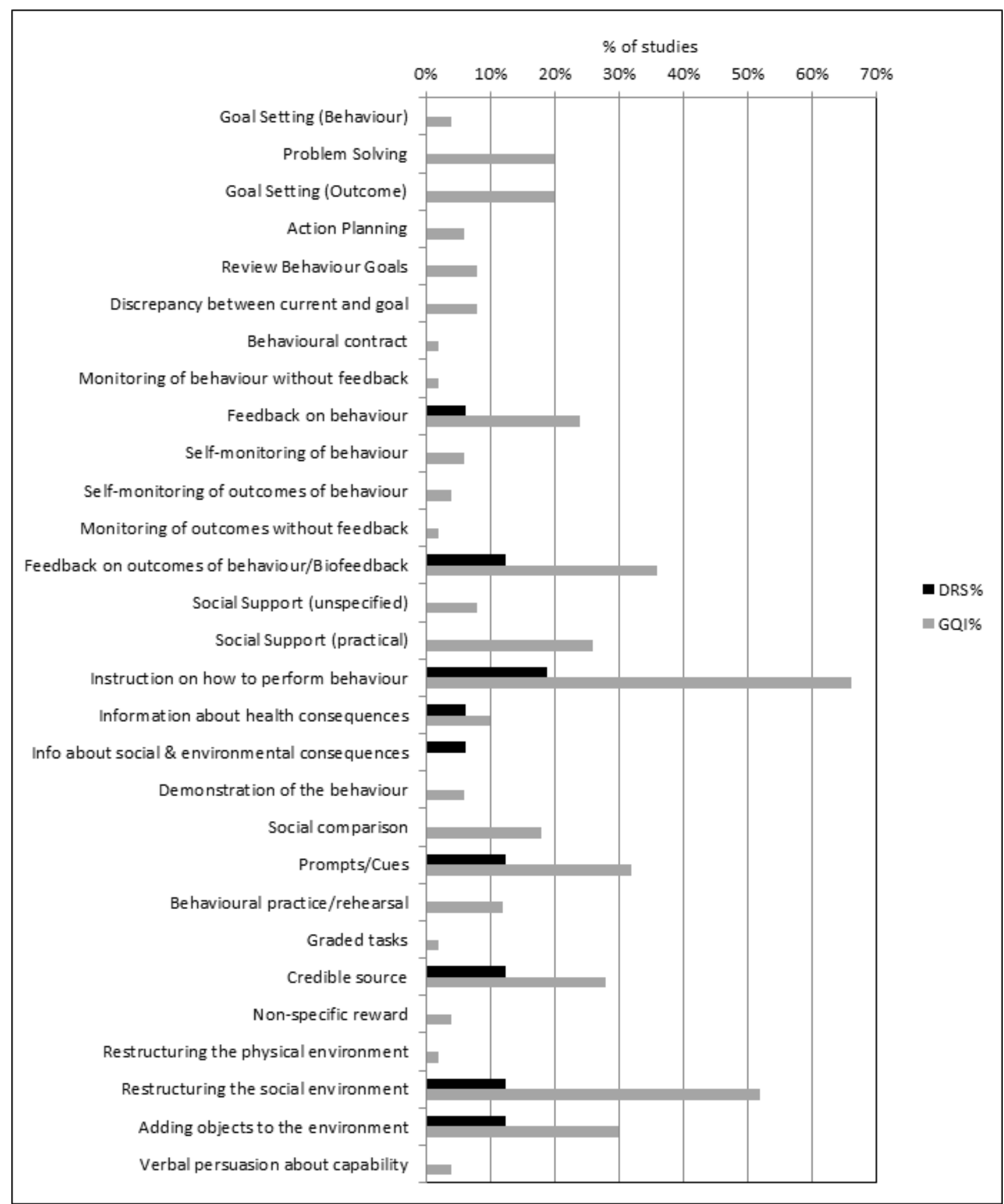

For studies comparing any intervention to usual care, most studies provided no description of usual care, which precluded coding of the comparator arm.

\section{Outcome measures}

In $12(75 \%)$ of the 16 studies where the primary target of the intervention was to improve attendance for DRS, the outcome was a dilated fundus examination conducted by an ophthalmologist or 
optometrist during the follow-up period post-intervention (median follow-up 12 months). The fundus examination was confirmed by a medical record audit, health claims database, or an eye-care professional confirmed examination. In four studies (25\%) DRS consisted of screening of digital retinal images.

Of the 50 studies where DRS attendance was reported as part of a general QI intervention, DRS was usually listed as part of a number of processes of care based on diabetes guideline recommendations. DRS was variously described as a dilated fundus examination/diabetic eye exam/retinal exam/eye exam in 49 studies (98\%) and involved grading of retinal images in one study. DRS was confirmed by medical record audit, from claims databases or patient self-reports (both validated and unvalidated by an eye-care professional). The median duration of follow-up was 12 months (range 1 - 48 months).

In terms of economic outcomes, five studies reported a full economic evaluation (Davis 2010; Eccles 2007; Pizzi 2015; Prezio 2014; Walker 2008).Three of these were cost-effectiveness analyses
(Davis 2010; Prezio 2014; Walker 2008) and two were costconsequence analyses (Eccles 2007; Pizzi 2015). Nine studies were partial economic evaluations; five were resource-utilisation studies, (Clancy 2007; Frei 2014; Krein 2004; McCall 2011; Piette 2001), while four were cost-outcome descriptions (Adair 2013; Frijling 2002; Litaker 2003; Wagner 2001). We could not retrieve the full text of one of the cost-effectiveness studies, but the abstract provided some information required for the review alongside the clinical-effectiveness report (Davis 2010).

\section{Excluded studies}

See Characteristics of excluded studies.

\section{Risk of bias in included studies}

We conducted 'Risk of bias' assessment using the Cochrane EPOC 'Risk of bias' tool. Figure 3 and Figure 6 summarise the risks of bias. Overall, we judged trials to be at low or unclear risk of bias for most of the bias domains. We provide support for each judgement in the Characteristics of included studies tables.

\section{Figure 6. Risk of bias graph: review authors' judgements about each risk of bias item presented as percentages across all included studies.}

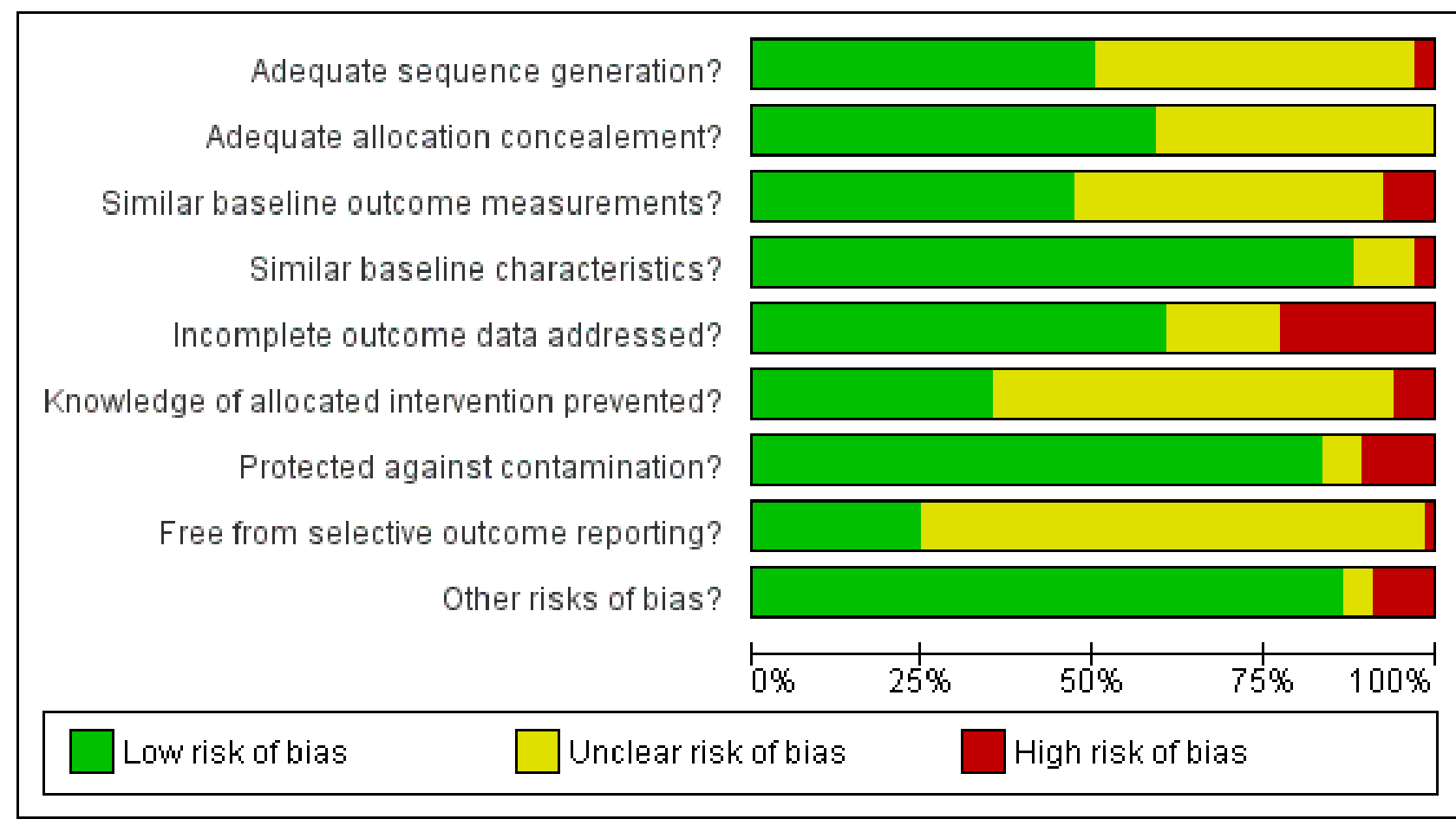

The studies that reported economic outcomes are a subset of the studies included in the review, and the risks of bias of these studies were very similar to the main body of included studies. With respect to the economic methodological quality, only five of the 14 included studies reported full economic evaluations (Davis 2010; Eccles 2007; Pizzi 2015; Prezio 2014; Walker 2008). One of these studies (Davis 2010) was published as an abstract and lacked important methodological details. Only three of the studies with full economic evaluations (Pizzi 2015; Prezio 2014; Walker 2008) reported a sensitivity analysis to explore changes in the costs and outcomes under different scenarios. Discounting in economic evaluations is necessary to adjust future costs and outcomes of an intervention to its present value, but was reported in only one of the full economic outcomes (Prezio 2014). Its use would have been appropriate in those other studies which had a stated followup of longer than 12 months (Eccles 2007; Frijling 2002; Krein 2004; Wagner 2008). We considered the methodological quality of the full economic evaluations to be moderate, while the partial economic evaluations by their nature lacked the methodological characteristics expected of an economic evaluation. Full details of the methodological quality assessment for each of the included economic evaluations are available in Table 4 and Table 5. 


\section{Allocation}

Thirty-three studies (50\%) reported using appropriate methods for random sequence allocation. Two studies (Gabbay 2006; McDermott 2001) described a non-random component in the sequence generation process and we judged them to be at a high risk of bias for this domain. The rest of the studies provided insufficient information about the sequence-generation process to judge risk of bias. We rated allocation concealment as adequate in 39 studies (59\%), either because the unit of allocation was by institution, team or professional and allocation was performed on all units at the start of the study, or a suitable method was used to conceal allocation.

\section{Blinding}

We rated four studies at a high risk of bias; Adair 2013, where retinopathy screening data were extracted from patient records by unmasked extractors, whose knowledge of allocation could have influenced outcome; Franco 2007, in which the general practitioners (GPs) in the intervention group provided the data on retinopathy screening; in Sonnichsen 2010, where masking was not possible and knowledge of being in the intervention or control group may have influenced the outcome; and Ward 1996, where one of the outcome assessors was the research nurse who conducted the interviews to obtain the outcome data in one arm of the trial, and was therefore unmasked.

\section{Incomplete outcome data}

We judged 15 studies (22.7\%) to be at a high risk of attrition bias, with attrition of 20\% or more (Dijkstra 2005; Franco 2007; Gabbay 2013; Harris 2005; Hermans 2013; Ilag 2003; Jacobs 2012; Jansink 2013; Kirwin 2010; Maljanian 2005; O'Connor 2005; Perria 2007; Sonnichsen 2010; Varney 2014; Wagner 2001).The remaining studies were either at low $(\mathrm{N}=40)$ or unclear $(\mathrm{N}=11)$ risk of bias for this domain.

\section{Selective reporting}

It was possible to judge if a study was free from selective outcome reporting in only 17 of the included studies (25.8\%), as the outcomes were consistent with a prospectively-published clinical trials registry entry or trial protocol. We were unable to assess selective reporting in the remainder, due to the lack of a study protocol or trial register entry, or in the case of studies where trial registration was performed retrospectively.

\section{Other potential sources of bias}

In five studies (7.6\%) there was a baseline imbalance in DRS attendance of $10 \%$ or more between intervention and control groups, and in seven studies (10.6\%) it was not possible to control for the possibility that the control group received the intervention.

\section{Effects of interventions}

See: Summary of findings for the main comparison Any quality improvement intervention compared to usual care for diabetic retinopathy screening; Summary of findings 2 Stepped quality improvement intervention compared to intervention alone for diabetic retinopathy screening

For details of the GRADE assessments, see Summary of findings for the main comparison and Summary of findings 2.

\section{Primary outcome}

See Summary of findings for the main comparison and Summary of findings 2 .

\section{One or more visits for diabetic retinopathy screening within a two-year period following implementation of the intervention}

All 66 trials provided data for this outcome. These consisted of two types of comparison: 56 of the 66 studies (85\%) compared an intervention against "current usual care", and $10(15 \%)$ compared a more intensive QI intervention or group of QI interventions against a less intensive intervention. Since these were addressing different questions, we conducted separate meta-analyses on the 56 and the 10 studies.

Thirty-one of the 66 trials (47\%) were cluster-RCTs. Only nine of these reported an ICC and the ICC reported typically did not relate specifically to DRS outcomes. Of the nine reporting an ICC, the most commonly reported value was 0.05 , and so this was the value we imputed for studies with no estimates of ICCs. The smallest value reported was 0.01 and the largest value was 0.2 . We ran a sensitivity analysis to investigate the impact on the computed effect estimates of using the lower and upper range values (see table below). 


\begin{tabular}{|c|c|c|c|c|c|c|c|c|c|}
\hline ICC & 0.05 & & & 0.01 & & & 0.2 & & \\
\hline Model & $\mathrm{RD}$ & $\mathrm{LCL}$ & UCL & $\mathrm{RD}$ & $\mathrm{LCL}$ & UCL & $\mathrm{RD}$ & $\mathrm{LCL}$ & UCL \\
\hline DRS & 0.17 & 0.11 & 0.22 & 0.17 & 0.11 & 0.22 & 0.17 & 0.11 & 0.22 \\
\hline General & 0.12 & 0.09 & 0.15 & 0.12 & 0.09 & 0.16 & 0.11 & 0.08 & 0.15 \\
\hline Combined & 0.12 & 0.10 & 0.14 & 0.13 & 0.11 & 0.15 & 0.12 & 0.10 & 0.14 \\
\hline
\end{tabular}

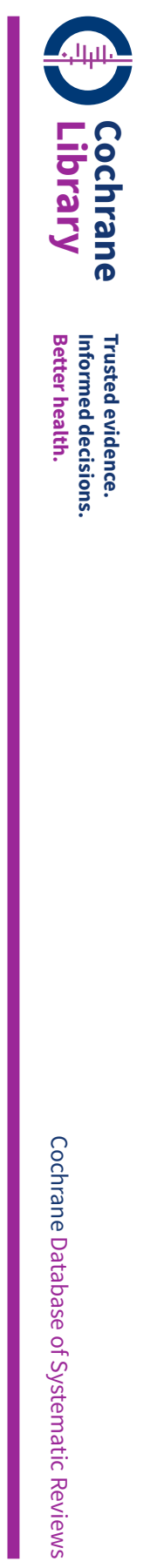


Abbreviations: RD: risk difference; LCL: lower limit; UCL: upper limit

\section{Comparison 1: Any QI intervention versus usual care}

Of the 56 studies which compared any intervention against usual care, $13(23 \%)$ evaluated interventions specifically targeting DRS. The remaining 43 (77\%) evaluated interventions directed towards improving the general quality of diabetes care (including DRS attendance). Although there was substantial heterogeneity in intervention effects $\left(1^{2}=93 \%\right), 48$ out of the 56 studies showed an improvement in DRS attendance. Since it may be argued that it is better to examine clinical differences in a meta-analysis rather than to use them as a reason for not conducting one, we computed pooled estimates for each of these subgroups. We adopted a random-effects model, which can accommodate statistical heterogeneity between studies by assuming that different studies have different true effect sizes, but we acknowledge that use of the random-effects model does not in it itself deal with heterogeneity. We assessed whether there was evidence of a subgroup effect and, since there was not $(P=0.15)$, we conducted all subsequent statistical analyses on the 56 studies. Overall, DRS attendance increased by $12 \%$ (risk difference (RD) $0.12,95 \%$ confidence interval (Cl) 0.10 to 0.14 ; low-certainty evidence) compared with usual care (Analysis 1.1 Figure 7). 
Figure 7. Forest plot of comparison: 1 Any quality improvement intervention compared to usual care, outcome: 1.1 Proportion of participants attending screening.

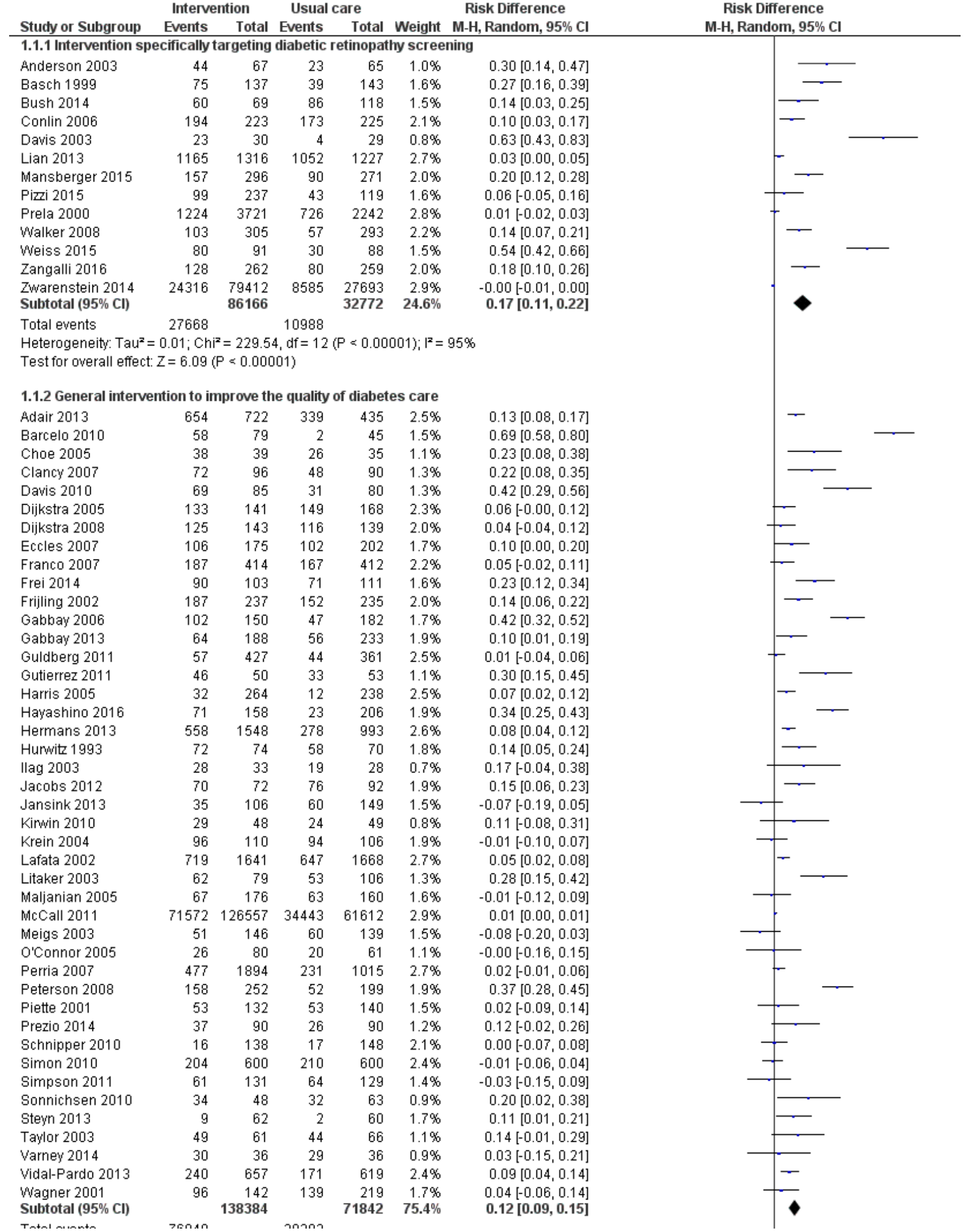


Figure 7. (Continued)

\begin{tabular}{|c|c|c|c|c|c|}
\hline $\begin{array}{l}\text { Wagner } 2001 \\
\text { Subtotal }(95 \% \mathrm{CI})\end{array}$ & 96 & $\begin{array}{r}142 \\
138384\end{array}$ & 139 & $\begin{array}{r}219 \\
71842\end{array}$ & $\begin{array}{r}1.7 \% \\
75.4 \%\end{array}$ \\
\hline \multicolumn{6}{|c|}{$\begin{array}{l}\text { Heterogeneity: } \operatorname{Tau}^{2}=0.01 ; \mathrm{Chi}^{2}=535.63, \mathrm{df}=42(\mathrm{P}<0.00001) ;\left.\right|^{2}=92 \% \\
\text { Test for overall effect: } Z=7.32(\mathrm{P}=0.00001)\end{array}$} \\
\hline Total $(\mathbf{9 5} \% \mathrm{Cl})$ & & 224550 & & 104614 & $100.0 \%$ \\
\hline $\begin{array}{l}\text { Total events } \\
\text { Heterogeneity: Tau } \\
\text { Test for overall effe } \\
\text { Test for subqroup }\end{array}$ & $\begin{array}{l}104608 \\
0.00 ; \mathrm{Chi} \\
Z=11.74 \\
\text { erences: }\end{array}$ & $\begin{array}{l}=776.5 \\
(P \leq 0.00 \\
C h i^{2}=2.0\end{array}$ & $\begin{array}{l}49371 \\
d f=55 \\
01)\end{array}$ & $(P=0.000$ & $001) ;\left.\right|^{2}=93 \%$ \\
\hline
\end{tabular}

There was some evidence of funnel plot asymmetry (Figure 8). Terrin 2003 has suggested, however, that the funnel plot may be

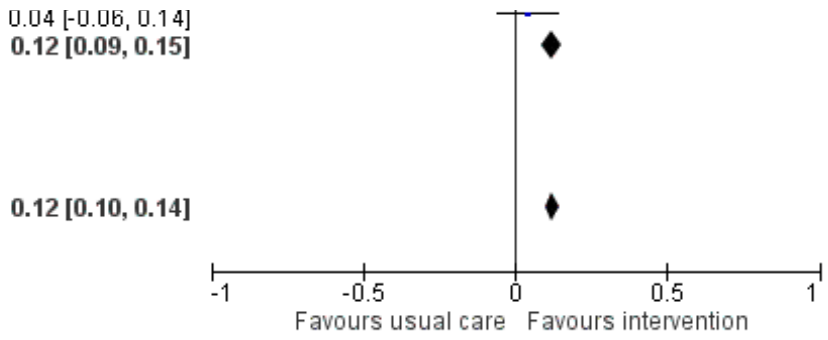

inappropriate for heterogeneous meta-analyses, so we did not downgrade our findings because of this.

Figure 8. Funnel plot of comparison: 1 Any quality improvement intervention compared to usual care, outcome: 1.1 Proportion of patients attending screening.

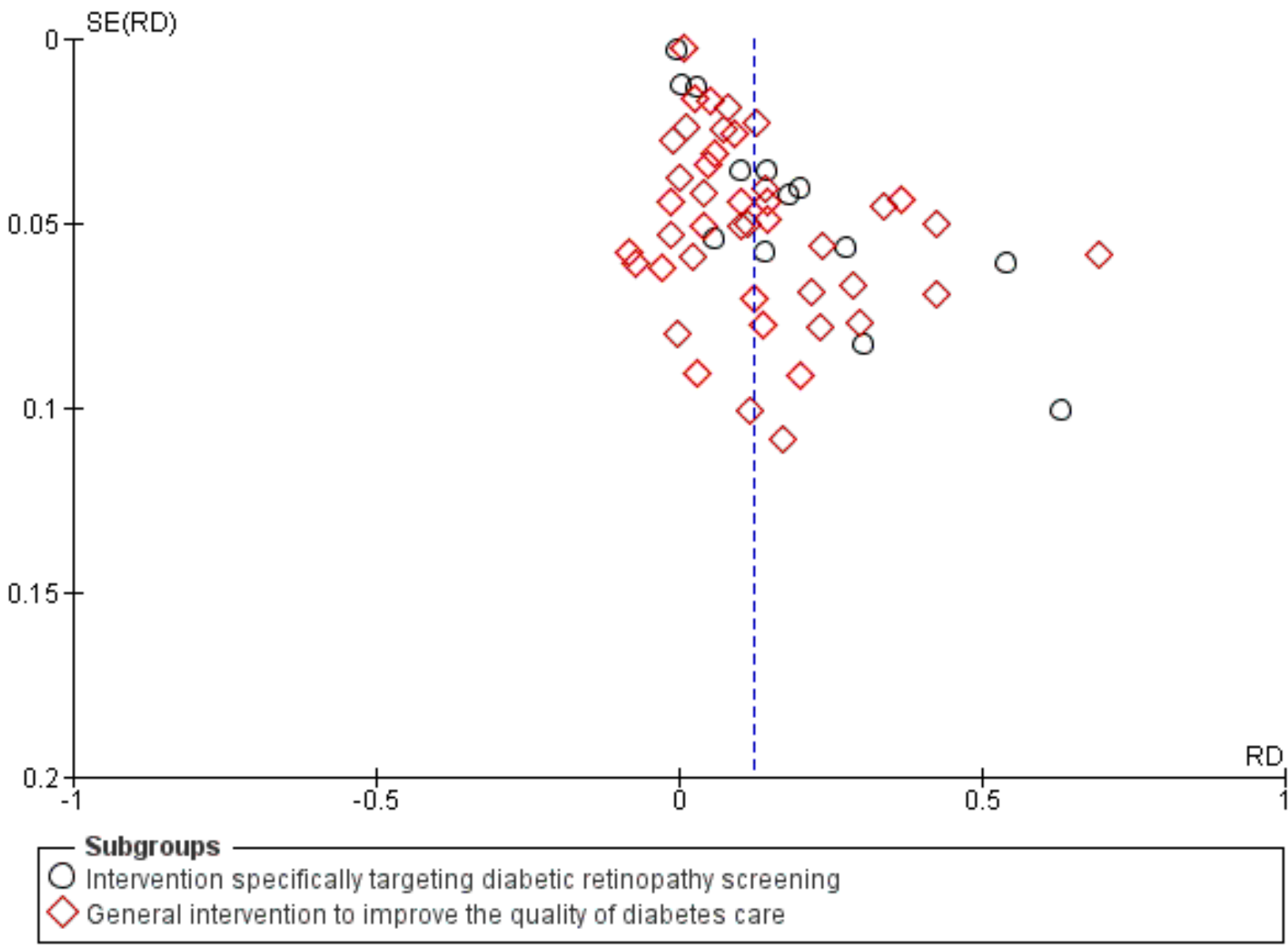

Comparison 2: More intensive (stepped) intervention versus less intensive intervention

Examples of studies in this comparison included: a tailored (individualised) versus a generic patient education newsletter; a comparison of audit and feedback to the healthcare professional compared to audit and feedback combined with a diabetes team outreach service. Ten studies contributed to this analysis (Analysis 2.1; Figure 9). Three (30\%) evaluated interventions specifically targeting DRS, while seven (70\%) evaluated interventions directed towards improving the general quality of diabetes care. In these studies DRS attendance increased by $5 \%$ (RD $0.05,95 \% \mathrm{Cl} 0.02$ to 0.09 ; moderate-certainty evidence) (Analysis 2.1). 
Figure 9. Forest plot of comparison: 2 Stepped quality improvement intervention compared to intervention alone (control), outcome: 2.1 Proportion of participants attending screening.

\begin{tabular}{|c|c|c|c|c|c|}
\hline \multirow[b]{2}{*}{ Study or Subgroup } & \multicolumn{2}{|c|}{ Stepped intervention } & \multicolumn{2}{|c|}{ Control } & \multirow[b]{2}{*}{ Weight } \\
\hline & Events & Total & Events & Total & \\
\hline \multicolumn{6}{|c|}{ 2.1.1 Intervention specifically targeting diabetic retinopathy screening } \\
\hline Ellish 2011 & 15 & 39 & 17 & 33 & $2.2 \%$ \\
\hline Halbert 1999 & 3666 & 9909 & 3403 & 9614 & $24.7 \%$ \\
\hline Rosenkranz 1996 & 49 & 66 & 19 & 37 & $3.0 \%$ \\
\hline Subtotal $(95 \% \mathrm{Cl}$ & & 10014 & & 9684 & \\
\hline Total events & 3730 & & 3439 & & \\
\hline \multicolumn{6}{|c|}{$\begin{array}{l}\text { Heterogeneity: } \text { Tau }^{2}=0.01 ; \mathrm{Chi}^{2}=6.27, \mathrm{df}=2(\mathrm{P}=0.04) ; \mathrm{I}^{2}=68 \% \\
\text { Test for overall effect: } Z=0.52(\mathrm{P}=0.60)\end{array}$} \\
\hline \multicolumn{6}{|c|}{ 2.1.2 General intervention to improve the quality of diabetes care } \\
\hline Dickinson 2014 & 53 & 253 & 20 & 162 & $12.5 \%$ \\
\hline Glasgow 2005 & 144 & 186 & 135 & 186 & $9.9 \%$ \\
\hline Herrin 2006 & 40 & 227 & 10 & 97 & $11.4 \%$ \\
\hline McClellan 2003 & 450 & 1142 & 424 & 1072 & $19.1 \%$ \\
\hline McDermott 2001 & 74 & 124 & 80 & 174 & $7.0 \%$ \\
\hline Ward 1996 & 96 & 231 & 39 & 124 & $8.0 \%$ \\
\hline Welch & 19 & 21 & 14 & 18 & $2.2 \%$ \\
\hline Subtotal $(95 \% \mathrm{Cl})$ & & 2184 & & 1833 & $70.1 \%$ \\
\hline Total events & 876 & & 722 & & \\
\hline \multicolumn{6}{|c|}{$\begin{array}{l}\text { Heterogeneity: } \operatorname{Tau}^{2}=0.00 ; \mathrm{Chi}^{2}=11.13, \mathrm{df}=6(P=0.08) ;\left.\right|^{2}=46 \% \\
\text { Test for overall effect: } Z=2.87(P=0.004)\end{array}$} \\
\hline Total $(95 \% \mathrm{Cl})$ & & 12198 & & 11517 & $100.0 \%$ \\
\hline Total events & 4606 & & 4161 & & \\
\hline \multirow{2}{*}{\multicolumn{6}{|c|}{$\begin{array}{l}\text { Heterogeneity: } \operatorname{Tau}^{2}=0.00 ; \mathrm{Chi}^{2}=20.32, \mathrm{df}=9(P=0.02) ;\left.\right|^{2}=56 \% \\
\text { Test for overall effect: } Z=2.88(P=0.004)\end{array}$}} \\
\hline & & & & & \\
\hline & & & & & \\
\hline
\end{tabular}

\section{Secondary outcomes}

\section{Ongoing adherence to DRS based on attendance for screening following the initial screening post-intervention}

It was not possible to extract data on ongoing adherence to DRS (based on attendance for screening following the initial screening post-intervention), since either it was not possible to identify unique screening episodes from pooled data reported at two time points, or in one study due to the intervention being offered to the comparator arm 18 months post-randomisation (Mansberger 2015).

\section{Economic outcomes}

Resources (staff time, equipment, consumables) required to deliver interventions to increase attendance for DRS

We graded each intervention between one (least resourceintensive) and five (most resource-intensive), or as zero (unable to determine), with a record of how the review author graded each study also provided. We developed an algorithm to derive the ordered rank. This mapped resource components and their intensity to the ordered rank. We incorporated the following resource components into the algorithm: face-to-face minutes; telephone calls; patient home visits; printed materials/software; training.

We then used the resource components and their intensity levels to extract the resource use required to deliver the interventions in all included studies. Two review authors (JL and EGR) conducted this independently. The percentage of studies for each resource grouping for the 56 studies comparing any intervention with usual care was as follows: $1=48.2 \% ; 2=10.7 \% ; 3=8.9 \% ; 4=19.6 \% ; 5=$ $12.6 \%$.
Risk Difference

Risk Difference M-H, Random, $95 \% \mathrm{Cl}$

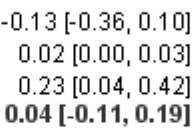

$\mathrm{Cl}$

$0.09[0.01,0.16]$

$0.05[-0.04,0.14]$

$0.07[-0.01,0.15]$

$-0.00[-0.04,0.04]$

$0.14[0.02,0.25]$

$0.10[-0.00,0.20]$

$0.13[-0.10,0.36]$

$0.06[0.02,0.11]$

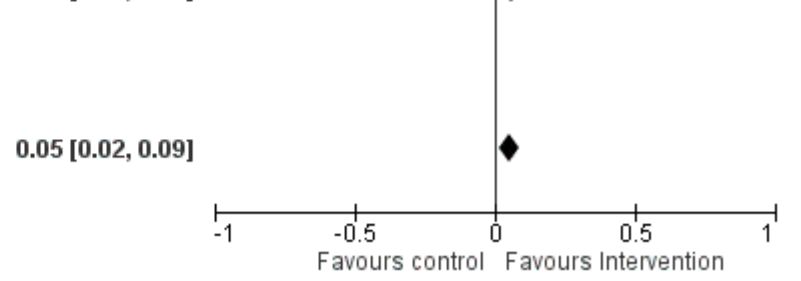

Costs of staff used to provide interventions; costs of treatment and care; cost of primary care; lost wages and lost productivity (work output)

We converted all reported costs to the 2016 British pound, and summarise them for each study in Table 2. Only two studies (Eccles 2007; Prezio 2014) reported both the direct and indirect costs (productivity loss) of the interventions. In all other studies, the costs of the interventions reported covered just the direct costs of providing that intervention. Five studies (Adair 2013; Clancy 2007; Frijling 2002; Prezio 2014; Pizzi 2015) reported the total direct costs of the interventions, but the resources they considered relevant and how they combined them to estimate total cost varied between studies. We report components of the total cost for each intervention in Table 2.

The types of resources included in the cost calculations for each study varied; hence, it is difficult to compare directly across the studies. The estimated training cost differed between the few studies that reported this information. In terms of the costs of treatment and care of diabetes, there was no obvious difference in the healthcare costs between the interventions and comparators in the studies that reported these data, primarily reflecting an absence of evidence. Further details on resources and costs from each included studies can be found in Table 2.

\section{Incremental cost-effectiveness ratio (ICER)}

Only three studies conducted in the USA (Davis 2010; Prezio 2014; Walker 2008) reported this outcome. Davis 2010 reported an incremental cost per QALY of GBP 13,154 over one year for a diabetes telecare intervention compared to no intervention. However, it is unclear what tool they used to estimate QALYs. Prezio 2014 used an established whole-disease model, the Archimedes Model simulator, to estimate the incremental cost per QALY. Using 
a discount rate of $3 \%$ and programme effectiveness at $100 \%$, the incremental cost per QALY was GBP 73,683 over five years, and GBP 261 over 20 years for the intervention (a culturallytailored diabetes education programme delivered by community health worker) compared with usual care. Prezio 2014 and Walker 2008 also reported an incremental cost-effectiveness ratio. In this study, the unit of effectiveness was the number of diabetic fundus examinations gained, which was associated with the number of diabetic retinopathies diagnosed. The incremental cost per dilated fundus examination gained for telephone intervention compared to the mailed/printed intervention was GBP 333. Pizzi 2015 reported a cost-effectiveness analysis with an incremental cost-effectiveness ratio for the telephone intervention of GBP 18.77 per additional patient attending a dilated fundus examination, compared with usual care. We did not calculate the ratio for the mailed intervention because it was dominated by usual care.

\section{Exploration of heterogeneity}

We detected substantial heterogeneity $\left(I^{2}>90 \%\right)$, which we investigated by subgroup analysis and meta-regression.

\section{Subgroup analysis}

Enough studies were available to investigate the effectiveness of nine out of the possible 12 QI components. Insufficient data were available to analyse 'continuous quality improvement', 'financial incentives' and 'facilitated relay' of information to clinicians. Interventions incorporating all nine QI components evaluated in the subgroup analysis were associated with improvements in DRS attendance, with higher pooled effect estimates for interventions directed at patients (promotion of self-management and patient education) or the organisation of the health system (team changes or the establishment of an electronic patient registry) (Table 6). Sufficient studies were available to investigate the effectiveness of interventions containing particular BCTs (including $10 \mathrm{BCTs}$ aimed at patients and seven aimed at healthcare professionals). Interventions incorporating all 17 BCTs included in the subgroup analysis were all shown to be effective in improving DRS attendance. For BCTs aimed at patients, we found higher pooled effect estimates for 'goal setting (outcome)' and 'credible source' and for healthcare professionals 'restructuring the social environment' and 'credible source' (Table 6). There were insufficient data to conduct the planned analysis on the variability of effect size according to population subgroups, and there were too few studies within each resource category to conduct a subgroup analysis of the relationship between effect size and resource intensity.

\section{Metaregressions}

Metaregression revealed some evidence of an association between effect size and baseline DRS attendance, with larger effects in studies with poorer screening attendance (Figure 10). The regression coefficient was $-0.208(-0.419$ to 0.004$)$. The residual 12 was still very high at $94 \%$. Because of regression to the mean, this association might be spurious, so we conducted a permutation test to allow for this (with 1000 permutations, $P=0.055$ ). A comparison between the effect sizes from studies at high risk of bias (defined for this purpose as high risk of bias in one or more domains) was slightly (but not statistically-significantly) higher than those at low risk of bias (regression coefficient 0.008 (-0.136 to 0.094)). Similarly, we found no association between study design (individual or cluster-RCT) and effect size (regression coefficient - 0.049 (-0.136 to $0.039), P=0.268)$, nor between resource intensity and effect size (regression coefficient $0.013(-0.0150 .042), P=0.356)$.

Figure 10. Bubble plot showing the relationship between the risk difference and baseline percentage screened

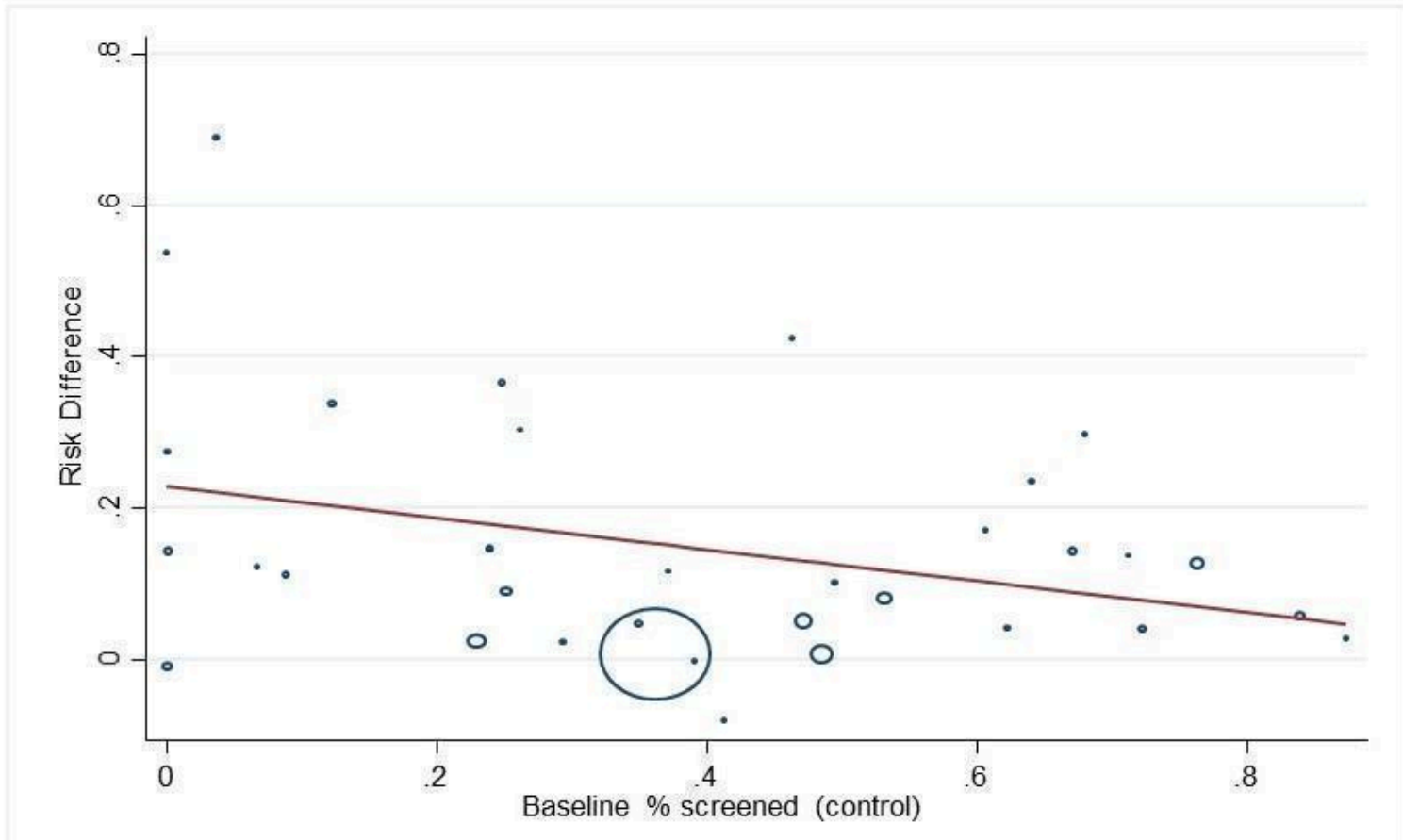


When component QI/BCTs were explored (comparing studies with the intervention to those studies without), there was some evidence of an association between the patient-targeted BCT 'goal setting (outcome)', with greater improvement in DRS attendance observed in studies with compared to those without this BCT (regression coefficient 0.162 (0.07 to 0.254$), P=0.001$ ). It should be noted that we made no adjustments for multiplicity in these investigations, so that results should be observed as hypothesisgenerating rather than confirmatory.

\section{DISCUSSION}

\section{Summary of main results}

This review identified 66 RCTs/cluster-RCTs that investigated the effectiveness of interventions to improve attendance for DRS. Fifty-six studies $(329,164$ participants) compared a variety of QI interventions to usual care. A meta-analysis of these studies found that QI intervention components that were aimed at patients, the healthcare professional or the healthcare system were associated with a $12 \%$ absolute increase in DRS attendance. In 13 of these studies, the QI intervention specifically targeted DRS and in 43 studies the intervention consisted of a general QI intervention to improve diabetes care. Although the pooled effect estimate was larger for DRS-targeted interventions compared to non-targeted interventions ( $17 \%$ increase in DRS attendance compared to $12 \%)$, this difference was not statistically significant.

Ten studies (23,715 participants) compared a less intensive intervention ('active' control) to a more intensive intervention. Three of these studies specifically targeted DRS and seven were general QI interventions. The aim of these studies was to determine whether stepping up the intensity of an intervention component, or introducing further components, would increase DRS. The pooled effect estimate for these studies was smaller, with a 5\% increase in DRS attendance in favour of the more intensive intervention, suggesting that it is possible to further enhance the effect size by using more intense interventions.

The main comparison in this review (any QI intervention versus usual care) was associated with substantial heterogeneity. We explored this by subgroup analysis and meta-regression. There was some evidence for larger effect sizes in populations with lower baseline DRS attendance; however, much of the observed heterogeneity was unexplained. Sufficient studies were available to investigate the impact of particular QI components or BCTs, to identify the active ingredients of the interventions. All 12 QI components, as defined by the modified EPOC taxonomy, were used in at least one study, and interventions were generally multifaceted, with two to three QI components per intervention arm. QI components targeting patients, healthcare professionals or the healthcare system were all effective in a subgroup analysis. A meta-regression comparing studies using particular QI components to those without them showed no statisticallysignificant difference between intervention components.

We were able to further describe interventions in terms of their component BCTs, which provides a level of granularity that is better suited to describing the content of the intervention. In a subgroup analysis, all frequently-used BCTs were effective in improving attendance, with pooled RDs ranging from 0.11 to 0.26 . A meta-regression found that interventions containing certain BCTs were more effective in improving DRS attendance, including: 'goal setting (outcome)' (regression coefficient (RC) $0.162,95 \% \mathrm{Cl} 0.070$ to $0.254, P=0.001$ ). There was some evidence for larger effect sizes in populations with lower baseline DRS attendance, (RC -0.208 , $95 \% \mathrm{Cl}-0.419$ to $0.004, \mathrm{P}=0.054)$. However much of the observed heterogeneity was unexplained.

We found no studies reporting our secondary outcome measure of ongoing adherence to DRS following the initial screening appointment post-intervention, and no data on the relative effectiveness of interventions in particular population subgroups, e.g. socioeconomic characteristics.

Fourteen studies reporting economic outcomes were included in the review. However, only five of these were full economic evaluations. Overall, we found that there is insufficient evidence to draw robust conclusions about the relative cost effectiveness of the interventions compared to each other or against usual care. QI components aimed at patients directly appeared to be more resource-intensive compared with those aimed at healthcare professionals, with the exception of establishing an electronic patient registry, although there would be economies of scale in that there are high set-up costs but the ongoing running costs would be comparatively low.

\section{Overall completeness and applicability of evidence}

To our knowledge only two countries in the world (UK and Iceland) have introduced a nationwide systematic screening programme for diabetic retinopathy. In all other countries screening remains opportunistic. Although an annual or biennial retinal examination is recommended in diabetes clinical practice guidelines in many countries, screening attendance is often suboptimal. Most of the trials included in this review (76\%) involved general QI interventions for diabetes care and enrolled patients not achieving diabetes-relevant quality indicators, including DRS. The pooled analysis for any QI intervention compared to usual care showed that both DRS-targeted and general QI interventions were effective in improving screening attendance, particularly in populations with poor baseline screening attendance. However, the presence of substantial unexplained heterogeneity and the lack of data on the effect of the intervention on particular population subgroups means that there remains some uncertainty about the size of the anticipated increase in screening attendance.

Although potential harms associated with other forms of health screening are well documented, we did not formally include adverse effects/harms as an outcome in this review, since the risk of an adverse outcome associated with retinopathy screening is low. However, none of the included studies reported adverse outcomes.

\section{Quality of the evidence}

Overall we judged the certainty of the evidence to be low, using GRADE. We downgraded the evidence by two levels due to serious inconsistency of findings. We decided a priori to use a randomeffects model to estimate the pooled RDs across studies, which weights studies relatively more equally than in a fixed-effect model. Given there was some evidence for larger effect sizes in smaller studies, our random-effects estimate of the intervention effect is more beneficial than would have been obtained using a fixed-effect model. 
For many domains, it was not possible to judge the risk of bias due to poor reporting. For example, since many of the RCTs did not have a prospectively-published protocol, it was not possible to make a judgement as to whether outcomes were selectively reported. A subgroup analysis found that, although studies at high risk of bias had slightly higher effect estimates compared to those at low risk of bias, this difference was not statistically significant. The consensus of the review team was not to downgrade the certainty of the evidence for risk of bias.

Of the 22 potential 'economic' studies identified by the review team, 14 were eligible for the review as partial or full economic evaluations. We judged the certainty of the economic evidence to be low, using GRADE. We downgraded due to inconsistency across different elements of the economic outcomes. We also identified publication bias in two of the eight excluded studies. These studies failed to report the planned economic evaluations, as they found no evidence of intervention effectiveness. Such an approach could be considered as selective outcome reporting, such that potentially negative economic findings are not reported. This phenomenon of a reporting bias has been recognised previously, where studies with unfavourable effectiveness results are not published or are published later in low-impact journals. Furthermore, analytically such an approach is substandard, as these studies conflate absence of evidence with a finding of evidence of absence (of an effect). We also found evidence of publication bias by inspection of a funnel plot, but this was difficult to assess in the presence of such considerable heterogeneity.

Most of the economic evaluations had limitations in their reporting, with few providing a breakdown of the costs associated with delivering the different components of the intervention. There was also insufficient evidence to show whether part of the direct costs of the intervention and care may be offset by reduced productivity costs. However, it is important to note that an expected finding of an effective intervention would be gains in health and reductions in the costs of treating diabetes. The overall methodological quality of the included economic studies was mixed. The partial economic evaluations identified, by their nature lacked the methodological characteristics expected of an economic evaluation. We rated the methodological quality of the full economic evaluations as moderate.

Many of our studies did not report ICC values. We used the data that were provided to allow an estimation of an "average ICC", which we then applied to the studies not reporting ICCs. Since this was an imputation, we wished to explore the impact that using other values of ICC would have, and thus repeated our analysis using the upper and lower values of ICC that had been observed. Varying in this fashion did not materially impact upon our estimates of RD.

\section{Potential biases in the review process}

We judged many domains as having an 'unclear' risk of bias, due to poor reporting. Although we contacted all authors to request further information on intervention content, we did not formally ask for all of the necessary information to make a more informed judgement across all bias domains.

Coding of intervention content was challenging, given the paucity of primary data sources, although in some cases (approximately $17 \%)$ this was offset by obtaining further information from researchers on intervention content, who also provided materials used in delivering the interventions. We were not able to assess the impact of some QI intervention components due to too few trials being available for our subgroup and meta-regression analyses. Furthermore, we could not control for all potential confounding factors. Given the complexity of the interventions which incorporated multiple QI components, it is likely that other covariates may have interacted synergistically or antagonistically with the intervention under investigation. The short duration of the included RCTs (typically 12 months or less) or the failure to report individual screening episodes meant that we were unable to assess the effect of $Q$ I interventions on ongoing DRS attendance.

\section{Agreements and disagreements with other studies or reviews}

Only one previous systematic review (Zhang 2007) has investigated the effectiveness of interventions to increase the uptake of DRS. Although this review included 48 studies, only 12 of these were RCTs. The authors similarly concluded that a variety of interventions can be effective in improving screening uptake, including; increasing patient and provider awareness of diabetic retinopathy, introducing a computer-based registration/reminder programme, and developing a community-based healthcare system.

Compared to the paucity of systematic reviews of the impact of interventions to improve DRS outcomes, many reviews have evaluated the impact of general QI interventions to improve the overall quality of diabetes care (Worswick 2013). A recent systematic review published by members of the current team (Tricco 2012) included 48 cluster-RCTs and 94 patient RCTs, and found improvements in many important quality outcomes for patients with diabetes. A meta-analysis of a subset of 23 RCTs reported an increased uptake of retinopathy screening (RR 1.22, $95 \% \mathrm{Cl} 1.13$ to 1.32 ).

\section{AUTHORS' CONCLUSIONS}

\section{Implications for practice}

The results of this review provide evidence that quality improvement (QI) interventions targeting patients, healthcare professionals or the healthcare system are associated with meaningful improvements in DRS attendance compared to usual care. There was no statistically-significant difference between interventions specifically aimed at DRS and those which were part of a general QI strategy for improving diabetes care. This is an important finding, because of the additional benefits of general QI interventions in terms of improving glycaemic control, vascular risk management and screening for other microvascular complications. It is likely that further (but smaller) improvements in DRS attendance can also be achieved by increasing the intensity of a particular QI component or adding further components.

One of the main objectives of the review was to identify the 'active' components of successful interventions by using validated taxonomies to describe the content of the interventions. All of the QI components as defined by the modified EPOC taxonomy were associated with improvements in DRS attendance. To better characterise intervention content we coded the interventions in terms of patient and provider behaviour change techniques (BCTs). For BCTs aimed at patients, we found higher effect estimates for interventions incorporating goal setting, and for healthcare 
professionals, interventions involving environmental restructuring. However, only $42 \%$ of the 93 possible BCTs were reported in the included interventions. Although not all BCTs in the BCT taxonomy might be appropriate for DRS, the findings of this review suggest that there may be opportunities to assess the potential of additional BCTs in future trials of novel interventions to improve screening attendance.

\section{Implications for research}

The review highlighted a number of gaps within the evidence base. There was limited evidence on the relative effectiveness of QI interventions in particular population subgroups according to demographic characteristics that could have an impact on health equity, e.g. ethnicity, level of education, or socioeconomic status. Moreover, none of the included studies were carried out in low- or middle-income countries. Further research is also needed on the cost effectiveness of QI interventions to improve DRS attendance.

Most of the included studies, whether targeting DRS or general QI strategies for diabetes care, enrolled patients not achieving diabetes-relevant quality indicators. For example, five studies specifically targeting DRS recruited exclusively patients who were not meeting guideline recommendations for screening. It is not clear whether the interventions would be as effective in populations with higher screening attendance (more than $80 \%$ ). There was some evidence from our meta-regression analysis that the effectiveness of the intervention is negatively correlated with baseline DRS attendance.

Although we have been able to show that interventions containing particular BCTs have a greater likelihood of success, given the multicomponent nature of interventions it is likely that the presence of other BCTs or other effect modifiers in the intervention arm may also be having an impact on effectiveness. The analysis conducted as part of this review did not attempt to fully isolate the impact of individual QI/BCT components. Further research is needed to identify which components of interventions or combinations of components can optimally improve DRS attendance at an acceptable cost.

\section{ACKNOWLEDGEMENTS}

We wish to acknowledge the 'What Works to Increase Attendance for Diabetic Retinopathy Screening? An Evidence sYnthEsiS (WIDeREyeS)' Project Stakeholder Advisory Group for their input to the development of the protocol for this review.

We thank Gianni Virgili, Luciana Ballini, Jemma Hudson, Noemi Lois and Jacqueline Ramke for their comments on the protocol or review. We acknowledge Jennifer Evans and Anupa Shah from Cochrane Eyes and Vision Group (CEV) for assisting with the preparation of this review. We thank Iris Gordon, Information Specialist for CEV, for developing the electronic search strategy. 


\section{RE F E R E N C E S}

\section{References to studies included in this review}

Adair 2013 \{published and unpublished data\}

Adair R, Wholey DR, Christianson J, White KM, Britt H, Lee S. Improving chronic disease care by adding laypersons to the primary care team: a parallel randomized trial. Annals of Internal Medicine 2013;159(3):176-84.

\section{Anderson 2003 \{published data only\}}

Anderson RM, Musch DC, Nwankwo RB, Wolf FM, Gillard ML, Oh MS, et al. Personalized follow-up increases return rate at urban eye disease screening clinics for African Americans with diabetes: Results of a randomized trial. Ethnicity and Disease 2003;13(1):40-6.

\section{Barcelo 2010 \{published data only\}}

Barcelo A, Cafiero E, De Boer M, Mesa AE, Lopez MG, Jimenez RA, et al. Using collaborative learning to improve diabetes care and outcomes: the VIDA project. Primary Care Diabetes 2010;4(3):145-53.

\section{Basch 1999 \{published data only\}}

Basch CE, Walker EA, Howard CJ, Shamoon H, Zybert P. The effect of health education on the rate of ophthalmic examinations among African Americans with diabetes mellitus. American Journal of Publlic Health 1999; Vol. 89, issue 12:1878-82.

\section{Bush 2014 \{published data only\}}

Bush K, Thomas R, Raymond NT, Sankar S, Barker PJ, O'Hare JP. Cluster randomised controlled trial evaluation of a Link Worker-delivered intervention to improve uptake of diabetic retinopathy screening in a South Asian population. Diabetes and Vascular Disease Research 2014;11(4):294-7.

\section{Choe 2005 \{published data only\}}

Choe HM, Mitrovich S, Dubay D, Hayward RA, Krein SL, Vijan S. Proactive case management of high-risk patients with type 2 diabetes mellitus by a clinical pharmacist: a randomized controlled trial. American Journal of Managed Care 2005;11(4):253-60

\section{Clancy 2007 \{published data only\}}

Clancy DE, Huang P, Okonofua E, Yeager D, Magruder KM. Group visits: promoting adherence to diabetes guidelines. Journal of General Internal Medicine 2007;22(5):620-4.

\section{Conlin 2006 \{published data only\}}

Conlin PR, Fisch BM, Cavallerano AA, Cavallerano JD, Bursell SE, Aiello LM. Nonmydriatic teleretinal imaging improves adherence to annual eye examinations in patients with diabetes. Journal of Rehabilitation Research and Development 2006;43(6):733-9.

\section{Davis 2003 \{published data only\}}

Davis RM, Fowler S, Bellis K, Pockl J, Al Pakalnis V, Woldorf A. Telemedicine improves eye examination rates in individuals with diabetes: a model for eye-care delivery in underserved communities. Diabetes Care 2003;26:2476.
Davis 2010 \{published data only\}

Davis R, Mayer-Davis EJ. Cost effectiveness of a telehealthbased diabetes self-management (DSME) intervention in a rural community. Diabetes 2011;60:A325-6.

* Davis RM, Hitch AD, Salaam MM, Herman WH, ZimmerGaller IE, Mayer-Davis EJ. TeleHealth improves diabetes selfmanagement in an underserved community: diabetes TeleCare. Diabetes Care 2010;33:1712-7.

\section{Dickinson 2014 \{published data only\}}

Dickinson WP, Dickinson LM, Nutting PA, Emsermann CB, Tutt B, Crabtree $\mathrm{BF}$, et al. Practice facilitation to improve diabetes care in primary care: a report from the EPIC randomized clinical trial. Annals of Family Medicine 2014;12(1):8-16.

\section{Dijkstra 2005 \{published data only\}}

Dijkstra RF, Braspenning JC, Huijsmans Z, Akkermans RP, Van Ballegooie E, Ten Have P, et al. Introduction of diabetes passports involving both patients and professionals to improve hospital outpatient diabetes care. Diabetes Research and Clinical Practice 2005;68(2):126-34

Dijkstra 2008 \{published data only\}

Dijkstra R, Braspenning J, Grol R. Implementing diabetes passports to focus practice reorganization on improving diabetes care. International Journal for Quality in Health Care 2008;20(1):72-7.

\section{Eccles 2007 \{published data only\}}

Eccles MP, Whitty PM, Speed C, Steen IN, Vanoli A, Hawthorne GC, et al. A pragmatic cluster randomised controlled trial of a Diabetes REcall And Management system: the DREAM trial. Implementation Science 2007;2:6.

Ellish 2011 \{published and unpublished data\} Ellish NJ, Royak-Schaler R, Higginbotham EJ. Tailored and targeted interventions to encourage dilated fundus examinations in older African Americans. Archives of Ophthalmology 2011;129(12):1592-8.

\section{Franco 2007 \{published data only\}}

Franco JM, De Chazournes P, Falcoff $\mathrm{H}$. Impact of peer visits [Impact des visites de pairs]. Revue du Praticien 2007;57:1211-7.

\section{Frei 2014 \{published data only\}}

Frei A, Chmiel C, Schlapfer H, Birnbaum B, Held U, Steurer J, et al. The Chronic CARe for diAbeTes study (CARAT): a cluster randomized controlled trial. Cardiovascular Diabetology 2010;9:23.

* Frei A, Senn O, Chmiel C, Reissner J, Held U, Rosemann T. Implementation of the chronic care model in small medical practices improves cardiovascular risk but not glycemic control. Diabetes Care 2014;37(4):1039-47.

Frijling 2002 \{published data only\}

Frijling BD, Lobo CM, Hulscher ME, Akkermans RP, Braspenning JC, Prins A, et al. Multifaceted support to improve 
clinical decision making in diabetes care: a randomized controlled trial in general practice. Diabetic Medicine 2002;19(10):836-42.

\section{Gabbay 2006 \{published data only\}}

Gabbay RA, Lendel I, Saleem TM, Shaeffer G, Adelman AM, Mauger DT, et al. Nurse case management improves blood pressure, emotional distress and diabetes complication screening. Diabetes Research and Clinical Practice 2006;71(1):28-35.

\section{Gabbay 2013 \{published data only\}}

* Gabbay RA, Añel-Tiangco RM, Dellasega C, Mauger DT, Adelman A, Van Horn DH. Diabetes nurse case management and motivational interviewing for change (DYNAMIC): results of a 2year randomized controlled pragmatic trial. Journal of Diabetes 2013;5(3):349-57.

Stuckey HL, Dellasega C, Graber NJ, Mauger DT, Lendel I, Gabbay RA. Diabetes nurse case management and motivational interviewing for change (DYNAMIC): study design and baseline characteristics in the Chronic Care Model for type 2 diabetes. Contemporary Clinical Trials 2009;30(4):366-74.

\section{Glasgow 2005 \{published data only\}}

Glasgow RE, Nutting PA, King DK, Nelson CC, Cutter G, Gaglio B, et al. A practical randomized trial to improve diabetes care. Journal of General Internal Medicine 2004;19(12):1167-74.

* Glasgow RE, Nutting PA, King DK, Nelson CC, Cutter G, Gaglio B, et al. Randomized effectiveness trial of a computerassisted intervention to improve diabetes care. Diabetes Care 2005;28(1):33-9.

\section{Guldberg 2011 \{published data only\}}

Guldberg TL, Vedsted P, Kristensen JK, Lauritzen T. Improved quality of Type 2 diabetes care following electronic feedback of treatment status to general practitioners: a cluster randomized controlled trial. Diabetic Medicine 2011;28(3):325-32.

\section{Gutierrez 2011 \{published data only\}}

Gutierrez N, Gimple NE, Dallo FJ, Foster BM, Ohagi EJ. Shared medical appointments in a residency clinic: an exploratory study among Hispanics with diabetes. American Journal of Managed Care 2011;17 (6 Spec No):e212-4.

\section{Halbert 1999 \{published data only\}}

Halbert RJ, Leung KM, Nichol JM, Legorreta AP. Effect of multiple patient reminders in improving diabetic retinopathy screening. A randomized trial. Diabetes Care 1999;22(5):752-5.

\section{Harris 2005 \{published data only\}}

* Harris SB, Leiter LA, Webster-Bogaert S, Van DM, O'Neill C. Teleconferenced educational detailing: diabetes education for primary care physicians. Journal of Continuing Education in the Health Professions 2005;25(2):87.

Harris SB, Worrall G, Macaulay A, Norton P, Webster-Bogaert S, Donner A, et al. Diabetes management in Canada: baseline results of the group practice diabetes management study. Canadian Journal of Diabetes 2006;30(2):131-7.

\section{Hayashino 2016 \{published data only\}}

* Hayashino Y, Suzuki H, Yamazaki K, Goto A, Izumi K, Noda M. A cluster randomized trial on the effect of a multifaceted intervention improved the technical quality of diabetes care by primary care physicians: The Japan Diabetes Outcome Intervention Trial-2 (J-DOIT2). Diabetic Medicine 2016;33(5):599-608.

Izumi K, Hayashino Y, Yamazaki K, Suzuki H, Ishizuka N, Kobayashi M, et al. Multifaceted intervention to promote the regular visiting of patients with diabetes to primary care physicians: Rationale, design and conduct of a clusterrandomized controlled trial. The Japan Diabetes Outcome Intervention Trial-2 study protocol. Diabetology International 2010;1(2):83-9.

\section{Hermans 2013 \{published data only\}}

Hermans MP, Elisaf M, Michel G, Muls E, Nobels F, Vandenberghe $\mathrm{H}$, et al. Benchmarking is associated with improved quality of care in type 2 diabetes: the OPTIMISE randomized, controlled trial. Diabetes Care 2013;36(11):3388-95.

\section{Herrin 2006 \{published data only\}}

Herrin J, Nicewander DA, Hollander PA, Couch CE, Winter FD, Haydar ZR, et al. Effectiveness of diabetes resource nurse case management and physician profiling in a fee-for-service setting: a cluster randomized trial. Proceedings 2006;19(2):95-102.

\section{Hurwitz 1993 \{published data only\}}

Hurwitz B, Goodman C, Yudkin J. Prompting the clinical care of non-insulin dependent (type II) diabetic patients in an inner city area: one model of community care. BMJ 1993;306(6878):624-30.

\section{Ilag 2003 \{published data only\}}

Ilag LL, Martin CL, Tabaei BP, Isaman DJ, Burke R, Greene DA, et al. Improving diabetes processes of care in managed care. Diabetes Care 2003;26(10):2722-7.

\section{Jacobs 2012 \{published data only\}}

Jacobs M, Sherry PS, Taylor LM, Amato M, Tataronis GR, Cushing G. Pharmacist Assisted Medication Program Enhancing the Regulation of Diabetes (PAMPERED) study. Journal of the American Pharmacists Association 2012;52(5):613-21.

\section{Jansink 2013 \{published data only\}}

Jansink R, Braspenning J, Keizer E, Van der Weijden T, Elwyn G, Grol R. No identifiable Hb1Ac or lifestyle change after a comprehensive diabetes programme including motivational interviewing: a cluster randomised trial. Scandinavian Journal of Primary Health Care 2013;31(2):119-27.

\section{Kirwin 2010 \{published data only\}}

Kirwin JL, Cunningham RJ, Sequist TD. Pharmacist recommendations to improve the quality of diabetes care: a randomized controlled trial. Journal of Managed Care Pharmacy 2010;16(2):104-13. 
Krein 2004 \{published data only\}

Krein SL, Klamerus ML, Vijan S, Lee JL, Fitzgerald JT, Pawlow A, et al. Case management for patients with poorly controlled diabetes: a randomized trial. American Journal of Medicine 2004;116(11):732-9.

\section{Lafata 2002 \{published data only\}}

Lafata JE, Baker AM, Divine GW, McCarthy BD, Xi H. The use of computerized birthday greeting reminders in the management of diabetes. Journal of General Internal Medicine 2002;17(7):521-30.

\section{Lian 2013 \{published data only\}}

Lian J, McGhee SM, Gangwani RA, Chan CK, Lam CL, Yap MK, et al. The impact of a co-payment on the cost-effectiveness of screening for diabetic retinopathy. Journal of Public Health (Oxford, England) 2016;38(4):782-92.

* Lian JX, McGhee SM, Gangwani RA, Hedley AJ, Lam CL, Yap MK, et al. Screening for diabetic retinopathy with or without a copayment in a randomized controlled trial: influence of the inverse care law. Ophthalmology 2013;120(6):1247-53.

\section{Litaker 2003 \{published data only\}}

Litaker D, Mion L, Planavsky L, Kippes C, Mehta N, Frolkis J. Physician - nurse practitioner teams in chronic disease management: the impact on costs, clinical effectiveness, and patients' perception of care. Journal of Interprofessional Care 2003;17(3):223-37.

\section{Maljanian 2005 \{published data only\}}

Maljanian R, Grey N, Staff I, Conroy L. Intensive telephone follow-up to a hospital-based disease management model for patients with diabetes mellitus. Disease Management 2005;8(1):15-25.

\section{Mansberger 2015 \{published data only\}}

Mansberger SL, Gleitsmann K, Gardiner S, Sheppler C, Demirel S, Wooten K, et al. Comparing the effectiveness of telemedicine and traditional surveillance in providing diabetic retinopathy screening examinations: a randomized controlled trial. Telemedicine Journal and E-Health 2013;19(12):942-8.

* Mansberger SL, Sheppler C, Barker G, Gardiner SK, Demirel S, Wooten $\mathrm{K}$, et al. Long-term Comparative Effectiveness of Telemedicine in Providing Diabetic Retinopathy Screening Examinations: A Randomized Clinical Trial. JAMA Ophthalmology 2015;133(5):518-25

\section{McCall 2011 \{published data only\}}

McCall N, Cromwell J. Results of the Medicare Health Support disease-management pilot program. The New England Journal of Medicine 2011;365(18):1704-12.

\section{McClellan 2003 \{published data only\}}

McClellan WM, Millman L, Presley R, Couzins J, Flanders WD. Improved diabetes care by primary care physicians: results of a group-randomized evaluation of the Medicare Health Care Quality Improvement Program (HCQIP). Journal of Clinical Epidemiology 2003;56(12):1210-7.

\section{McDermott 2001 \{published data only\}}

McDermott RA, Schmidt BA, Sinha A, Mills P. Improving diabetes care in the primary healthcare setting: a randomised cluster trial in remote Indigenous communities. The Medical Journal of Australia 2001;174(10):497-502.

Meigs 2003 \{published data only\}

Meigs JB, Cagliero E, Dubey A, Murphy-Sheehy P, Gildesgame C, Chueh $\mathrm{H}$, et al. A controlled trial of web-based diabetes disease management: the MGH diabetes primary care improvement project. Diabetes Care 2003;26(3):750-7.

O'Connor 2005 \{published data only\}

O'Connor PJ, Desai J, Solberg LI, Reger LA, Crain AL, Asche SE, et al. Randomized trial of quality improvement intervention to improve diabetes care in primary care settings. Diabetes Care 2005;28(8):1890-7.

\section{Perria 2007 \{published data only\}}

Perria C, Mandolini D, Guerrera C, Jefferson T, Billi P, Calzini V, et al. Implementing a guideline for the treatment of type 2 diabetics: results of a cluster-randomized controlled trial (CRCT). BMC Health Services Research 2007;7:79.

\section{Peterson 2008 \{published data only\}}

Peterson KA, Radosevich DM, O'Connor P, Nyman JA, Prineas RJ, Smith SA, et al. Improving diabetes care in practice. Diabetes Care 2008;31(12):2238-43.

\section{Piette 2001 \{published data only\}}

* Piette JD, Weinberger M, Kraemer FB, McPhee SJ. Impact of automated calls with nurse follow-up on diabetes treatment outcomes in a Department of Veterans Affairs Health Care System: a randomized controlled trial. Diabetes Care 2001;24(2):202-8

Piette JD, Weinberger M, McPhee SJ. The effect of automated calls with telephone nurse follow-up on patient-centered outcomes of diabetes care: a randomized, controlled trial. Medical Care 2000;38(2):218-30.

Pizzi 2015 \{published and unpublished data\}

Pizzi LT, Zangalli CS, Murchison AP, Hale N, Hark L, Dai Y, et al. Prospective randomized controlled trial comparing the outcomes and costs of two eyecare adherence interventions in diabetes patients. Applied Health Economics and Health Policy 2015;13(2):253-63.

\section{Prela 2000 \{published data only\}}

Prela CM, Smilie JG, McInerney MJ, Harwell TS, Helgerson SD. Direct mail intervention to increase retinal examination rates in Medicare beneficiaries with diabetes. American Journal of Medical Quality 2000;15(6):257-62.

Prezio 2014 \{published data only\}

* Prezio EA, Balasubramanian BA, Shuval K, Cheng D, Kendzor DE, Culica D. Evaluation of quality improvement performance in the Community Diabetes Education (CoDE) program for uninsured Mexican Americans: results of a randomized controlled trial. American Journal of Medical Quality 2014;29(2):124-34. 
Prezio EA, Cheng D, Balasubramanian BA, Shuval K, Kendzor DE, Culica D. Community Diabetes Education (CODE) for uninsured Mexican Americans: a randomized controlled trial of a culturally tailored diabetes education and management program led by a community health worker. Diabetes Research and Clinical Practice 2013;100(1):19-28.

Prezio EA, Pagan JA, Shuval K, Culica D. The Community Diabetes Education (CoDE) program: cost-effectiveness and health outcomes. American Journal of Preventive Medicine 2014;47(6):771-9.

\section{Rosenkranz 1996 \{published data only\}}

Rosenkranz S. Polaroid-fundus photography enhances patient compliance with screening for diabetic retinopathy. Diabetes und Stoffwechsel 1996;5:69-75.

\section{Schnipper 2010 \{published data only\}}

Schnipper JL, Linder JA, Palchuk MB, Yu DT, McColgan KE, Volk LA, et al. Effects of documentation-based decision support on chronic disease management. American Journal of Managed Care 2010;16(12 Suppl HIT):SP72-81.

\section{Simon 2010 \{published data only\}}

Simon SR, Trinacty CM, Soumerai SB, Piette JD, Meigs JB, Shi $P$, et al. Improving diabetes care among patients overdue for recommended testing: a randomized controlled trial of automated telephone outreach. Diabetes Care 2010;33(7):1452-3.

\section{Simpson 2011 \{published data only\}}

Simpson SH, Majumdar SR, Tsuyuki RT, Lewanczuk RZ, Spooner R, Johnson JA. Effect of adding pharmacists to primary care teams on blood pressure control in patients with type 2 diabetes: a randomized controlled trial. Diabetes Care 2011;34(1):20-6.

\section{Sonnichsen 2010 \{published data only\}}

Sonnichsen AC, Winkler H, Flamm M, Panisch S, Kowatsch P, Klima G, et al. The effectiveness of the Austrian disease management programme for type 2 diabetes: a clusterrandomised controlled trial. BMC Family Practice 2010;11:86.

\section{Steyn 2013 \{published data only\}}

Steyn K, Lombard C, Gwebushe N, Fourie JM, Everett-Murphy K, Zwarenstein $\mathrm{M}$, et al. Implementation of national guidelines, incorporated within structured diabetes and hypertension records at primary level care in Cape Town, South Africa: a randomised controlled trial. Global Health Action 2013;6:20796.

\section{Taylor 2003 \{published data only\}}

Taylor CB, Miller NH, Reilly KR, Greenwald G, Cunning D, Deeter $A$, et al. Evaluation of a nurse-care management system to improve outcomes in patients with complicated diabetes. Diabetes Care 2003;26(4):1058-63.

\section{Varney 2014 \{published and unpublished data\}}

Varney JE, Weiland TJ, Inder WJ, Jelinek GA. Effect of hospitalbased telephone coaching on glycaemic control and adherence to management guidelines in type 2 diabetes, a randomised controlled trial. Internal Medicine Journal 2014;44(9):890-7.
Vidal-Pardo 2013 \{published data only\}

Vidal-Pardo JI, Perez-Castro TR, Lopez-Alvarez XL, SantiagoPerez MI, Garcia-Soidan FJ, Muniz J. Effect of an educational intervention in primary care physicians on the compliance of indicators of good clinical practice in the treatment of type 2 diabetes mellitus [OBTEDIGA project]. International Journal of Clinical Practice 2013;67(8):750-8.

\section{Wagner 2001 \{published data only\}}

Wagner EH, Grothaus LC, Sandhu N, Galvin MS, McGregor M, Artz K, et al. Chronic care clinics for diabetes in primary care: a system-wide randomized trial. Diabetes Care 2001;24(4):695-700.

\section{Walker 2008 \{published data only\}}

Jones HL, Walker EA, Schechter CB, Blanco E. Vision is precious: a successful behavioral intervention to increase the rate of screening for diabetic retinopathy for inner-city adults. Diabetes Educator 2010;36(1):118-26.

Schechter CB, Basch CE, Caban A, Walker EA. Cost effectiveness of a telephone intervention to promote dilated fundus examination in adults with diabetes mellitus. Clinical Ophthalmology 2008;2(4):763-8.

* Walker EA, Schechter CB, Caban A, Basch CE. Telephone intervention to promote diabetic retinopathy screening among the urban poor. American Journal of Preventive Medicine 2008;34(3):185-91.

\section{Ward 1996 \{published data only\}}

Ward A, Kamien M, Mansfield F, Fatovich B. Educational feedback in the management of type 2 diabetes in general practice. Education for General Practice 1996;7:142-50.

\section{Weiss 2015 \{published data only\}}

Casten RJ, Brawer R, Haller JA, Hark LA, Henderer J, Leiby B, et al. Trial of a behavioral intervention to increase dilated fundus examinations in African-Americans aged over 65 years with diabetes. Expert Review of Ophthalmology 2011;6:593-601.

* Weiss DM, Casten RJ, Leiby BE, Hark LA, Murchison AP, Johnson $\mathrm{D}$, et al. Effect of behavioral intervention on dilated fundus examination rates in older African American individuals with diabetes mellitus a randomized clinical trial. JAMA Ophthalmology 2015;133(9):1005-12.

Welch 2011 \{published data only\}

Welch G, Allen NA, Zagarins SE, Stamp KD, Bursell SE, Kedziora RJ. Comprehensive diabetes management program for poorly controlled Hispanic type 2 patients at a community health center. Diabetes Educator 2011;37(5):680-8.

\section{Zangalli 2016 \{published data only\}}

Zangalli CS, Murchison AP, Hale N, Hark LA, Pizzi LT, Dai Y, et al. An education and telephone-based intervention to improve follow-up to vision care in patients with diabetes: a prospective, single-blinded, randomized trial. American Journal of Medical Quality 2016;31(2):156-61. 
Zwarenstein 2014 \{published data only\}

Grimshaw JM, Presseau J, Tetroe J, Eccles MP, Francis JJ, Godin G, et al. Looking inside the black box: results of a theorybased process evaluation exploring the results of a randomized controlled trial of printed educational messages to increase primary care physicians' diabetic retinopathy referrals [Trial registration number ISRCTN72772651]. Implementation Science 2014;9:86.

* Zwarenstein M, Shiller SK, Croxford R, Grimshaw JM, Kelsall D, Paterson JM, et al. Printed educational messages aimed at family practitioners fail to increase retinal screening among their patients with diabetes: a pragmatic cluster randomized controlled trial [ISRCTN72772651]. Implementation Science 2014;9:87.

\section{References to studies excluded from this review}

\section{Abraira 2003 \{published data only\}}

Abraira C, Duckworth W, McCarren M, Emanuele N, Arca D, Reda $D$, et al. Design of the cooperative study on glycemic control and complications in diabetes mellitus type 2: Veterans Affairs Diabetes Trial. Journal of Diabetes and its Complications 2003;17(6):314-22.

\section{Aleo 2015 \{published data only\}}

Aleo CL, Murchison AP, Dai Y, Hark LA, Mayro EL, Collymore B, et al. Improving eye care follow-up adherence in diabetic patients with ocular abnormalities: the effectiveness of patient contracts in a free, pharmacy-based eye screening. Public Health 2015;129(7):996-9.

\section{Alfadda 2011 \{published data only\}}

Alfadda AA, Bin-Abdulrahman KA, Saad HA, Mendoza CD, Angkaya-Bagayawa FF, Yale JF. Effect of an intervention to improve the management of patients with diabetes in primary care practice. Saudi Medical Journal 2011;32(1):36-40.

\section{Anderson 2003a \{published data only\}}

Anderson S, Broadbent DM, Swain JY, Vora JP, Harding SP. Ambulatory photographic screening for diabetic retinopathy in nursing homes. Eye 2003;17(6):711-6.

\section{Anderson 2010 \{published data only\}}

Anderson DR, Christison-Lagay J, Villagra V, Liu H, Dziura J. Managing the space between visits: a randomized trial of disease management for diabetes in a community health center. Journal of General Internal Medicine 2010;25(10):1116-22.

\section{Arora 2014 \{published data only\}}

Arora S, Peters AL, Burner E, Lam CN, Menchine M. Trial to examine text message-based mHealth in emergency department patients with diabetes (TExT-MED): a randomized controlled trial. Annals of Emergency Medicine 2014;63(6):745-54.

\section{Bellazzi 2004 \{published data only\}}

Bellazzi R, Arcelloni M, Ferrari P, Decata P, Hernando ME, Garcia A, et al. Management of patients with diabetes through information technology: tools for monitoring and control of the patients' metabolic behavior. Diabetes Technology and Therapeutics 2004;6(5):567-78.

Denig 2014 \{published data only\}

Denig P, Schuling J, Haaijer-Ruskamp F, Voorham J. Effects of a patient oriented decision aid for prioritising treatment goals in diabetes: pragmatic randomised controlled trial. BMJ 2014;349:g5651.

\section{Gangwar 2014 \{published data only\}}

Gangwar SS, Parimalakrishnan S, Monisha N, Singh SP. Impact of medication and psychological behavior assessment by community pharmacists in type 2 diabetes mellitus patients after hospital stay. International Research Journal of Pharmacy 2014;5:332-9.

\section{Gary 2004 \{published data only\}}

Gary TL, Batts-Turner M, Bone LR, Yeh HC, Wang NY, HillBriggs $F$, et al. A randomized controlled trial of the effects of nurse case manager and community health worker team interventions in urban African-Americans with type 2 diabetes. Controlled Clinical Trials 2004;25(1):53-66.

\section{Harris 2013 \{published data only\}}

Harris S, Paquette-Warren J, Roberts S, Fournie M, Thind A, Ryan BL, et al. Results of a mixed-methods evaluation of partnerships for health: a quality improvement initiative for diabetes care. Journal of the American Board of Family Medicine 2013;26(6):711-9

\section{Hazavehei 2010 \{published data only\}}

Hazavehei SM, Khani Jeihooni A, Hasanzadeh A, Amini S. The effect of educational program based on BASNEF model for eye care in non-insulin dependent diabetic patients. Journal of Research in Health Sciences 2010;10(2):81-90.

\section{Hollander 2005 \{published data only\}}

Hollander P, Nicewander D, Couch C, Winter D, Herrin J, Haydar Z, et al. Quality of care of Medicare patients with diabetes in a metropolitan fee-for-service primary care integrated delivery system. American Journal of Medical Quality 2005;20(6):344-52.

\section{Jones 2006 \{published data only\}}

Jones D, Curry W. Impact of a PDA-based diabetes electronic management system in a primary care office. American Journal of Medical Quality 2006;21(6):401-7.

Kuvaja-Kollner 2013 \{published data only\}

Kuvaja-Kollner V, Steffansson M, Kettunen A. Information and communications technology-supported diabetes prevention and self-care management: experiences from the EMOTIONAAL project in Finland. Journal of Diabetes Science and Technology 2013; Vol. 7, issue 1:287-8.

Lewis 2007 \{published data only\}

Lewis K, Patel D, Yorston D, Charteris D. A qualitative study in the United Kingdom of factors influencing attendance by patients with diabetes at ophthalmic outpatient clinics. Ophthalmic Epidemiology 2007;14(6):375-80. 
Maberley 2003 \{published data only\}

Maberley D, Walker H, Koushik A, Cruess A. Screening for diabetic retinopathy in James Bay, Ontario: a cost-effectiveness analysis. CMAJ 2003;168(2):160-4.

\section{Mangione 2006 \{published data only\}}

Mangione CM, Gerzoff RB, Williamson DF, Steers WN, Kerr EA, Brown AF, et al. The association between quality of care and the intensity of diabetes disease management programs. Annals of Internal Medicine 2006;145(2):107-16.

\section{Mazzuca 1988 \{published data only\}}

Mazzuca SA, Vinicor F, Cohen SJ, Norton JA, Fineberg NS, Fineberg SE, et al. The Diabetes Education Study: a controlled trial of the effects of intensive instruction of internal medicine residents on the management of diabetes mellitus. Journal of General Internal Medicine 1988;3(1):1-8.

\section{McCulloch 1998 \{published data only\}}

McCulloch DK, Price MJ, Hindmarsh M, Wagner EH. A population-based approach to diabetes management in a primary care setting: early results and lessons learned. Effective Clinical Practice 1998;1(1):12-22.

\section{Montori 2002 \{published data only\}}

Montori VM, Dinneen SF, Gorman CA, Zimmerman BR, Rizza RA, Bjornsen SS, et al. The impact of planned care and a diabetes electronic management system on community-based diabetes care: the Mayo Health System Diabetes Translation Project. Diabetes Care 2002;25(11):1952-7.

\section{Montori 2004 \{published data only\}}

Montori VM, Helgemoe PK, Guyatt GH, Dean DS, Leung TW, Smith SA, et al. Telecare for patients with type 1 diabetes and inadequate glycemic control: a randomized controlled trial and meta-analysis. Diabetes Care 2004;27(5):1088-94.

\section{Peters 1998 \{published data only\}}

Peters AL, Davidson MB. Application of a diabetes managed care program. The feasibility of using nurses and a computer system to provide effective care. Diabetes Care 1998;21(7):1037-43.

\section{Polak 2003 \{published data only\}}

Polak BC, Crijns H, Casparie AF, Niessen LW. Cost-effectiveness of glycemic control and ophthalmological care in diabetic retinopathy. Health Policy 2003;64(1):89-97.

\section{Rees 2013 \{published data only\}}

Rees G, Lamoureux EL, Nicolaou TE, Hodgson LA, Weinman J, Speight J. Feedback of personal retinal images appears to have a motivational impact in people with non-proliferative diabetic retinopathy and suboptimal $\mathrm{HbA1c}$ : findings of a pilot study. Diabetic Medicine 2013;30(9):1122-5.

\section{Samoutis 2010 \{published data only\}}

Samoutis GA, Soteriades ES, Stoffers HE, Philalithis A, Delicha EM, Lionis C. A pilot quality improvement intervention in patients with diabetes and hypertension in primary care settings of Cyprus. Family Practice 2010;27(3):263-70.

\section{Schectman 2004 \{published data only\}}

Schectman JM, Schorling JB, Nadkarni MM, Lyman JA, Siadaty MS, Voss JD. The effect of physician feedback and an action checklist on diabetes care measures. American Journal of Medical Quality 2004;19(5):207-13.

Shah 2014 \{published data only\}

Shah BR, Bhattacharyya O, Yu CH, Mamdani MM, Parsons JA, Straus SE, et al. Effect of an educational toolkit on quality of care: a pragmatic cluster randomized trial. PLoS Medicine 2014;11(2):e1001588.

\section{Shea 2006 \{published data only\}}

Shea S, Weinstock RS, Starren J, Teresi J, Palmas W, Field L, et al. A randomized trial comparing telemedicine case management with usual care in older, ethnically diverse, medically underserved patients with diabetes mellitus. Journal of the American Medical Informatics Association 2006;13(1):40-51.

Solorio 2015 \{published data only\}

Solorio R, Bansal A, Comstock B, Ulatowski K, Barker S. Impact of a chronic care coordinator intervention on diabetes quality of care in a community health center. Health Services Research 2015;50(3):730-49.

\section{Thoolen 2008 \{published data only\}}

Thoolen B, De Ridder D, Bensing J, Gorter K, Rutten G. Beyond Good Intentions: the development and evaluation of a proactive self-management course for patients recently diagnosed with type 2 diabetes. Health Education Research 2008;23(1):53-61.

\section{Wagner 2008 \{published data only\}}

Wagner H, Pizzimenti JJ, Daniel K, Pandya N, Hardigan PC. Eye on diabetes: a multidisciplinary patient education intervention. Diabetes Educator 2008;34(1):84-9.

\section{Weston 2008 \{published data only\}}

Weston CM, Sciamanna CN, Nash DB. Evaluating online continuing medical education seminars: evidence for improving clinical practices. American Journal of Medical Quality 2008;23(6):475-83.

\section{Young 2014 \{published data only\}}

Young H, Miyamoto S, Ward D, Dharmar M, Tang-Feldman Y, Berglund L. Sustained effects of a nurse coaching intervention via telehealth to improve health behavior change in diabetes. Telemedicine Journal and E-Health 2014;20(9):828-34.

\section{References to ongoing studies \\ ACTRN12614001110673 \{published data only\}}

ACTRN12614001110673. The Diabetes and Eye Health project: increasing eye examinations for adults newly diagnosed with type 2 diabetes [Development and evaluation of a psychoeducational leaflet to increase the rate of eye examinations for adults newly diagnosed with type 2 diabetes from the following two groups: 1 . early onset type 2 diabetes (aged 18-39 years), or 2. who live in rural and regional Victoria]. anzctr.org.au/Trial/ 
Registration/TrialReview.aspx?id=367127 (registered 20 October 2014).

\section{ISRCTN31439939 \{published data only\}}

ISRCTN31439939. The Kilimanjaro Diabetic Programme: the development of a sustainable regional eye health screening program to prevent blindness among diabetic patients due to diabetic retinopathy [A randomised controlled trial of screening strategies for diabetic retinopathy in Kilimanjaro region: a randomised controlled trial of the effect of a screening camera on uptake of screening for diabetic retinopathy (phase I) and a randomised controlled trial of the effect of mobile telephone text reminders of screening appointments on uptake of screening for diabetic retinopathy (phase II)]. www.isrctn.com/ ISRCTN31439939 (assigned 2 June 2011).

\section{ISRCTN87561257 \{published data only\}}

ISRCTN87561257. Individual risk-based screening for diabetic retinopathy [Introducing personalised risk-based intervals in screening for diabetic retinopathy: development, implementation and assessment of safety, cost-effectiveness and patient experience]. www.isrctn.com/ISRCTN87561257 (assigned 8 May 2014).

\section{NCT01212328 \{published data only\}}

NCT01212328. A multi-site, individually randomized, controlled translation trial of integrated and comprehensive care strategies to reduce cardiovascular disease (CVD) risk among 1,120 type 2 diabetes mellitus(T2DM) patients in south Asia (CARRS) [Developing and testing integrated, multi-factorial cardiovascular disease risk reduction strategies in south Asia (CARRS Translation Trial)]. clinicaltrials.gov/ct2/show/ NCT01212328 (first received 6 September 2010).

\section{NCT01351857 \{published data only\}}

NCT01351857. Diabetes care management compared to standard diabetes care in adolescents and young adults with type 1 diabetes (TransClin) [Multicentre randomized controlled trial of structured transition on diabetes care management compared to standard diabetes care in adolescents and young adults with type 1 diabetes]. clinicaltrials.gov/ct2/show/ NCT01351857 (first submitted 10 May 2011).

* Spaic T, Mahon J L, Hramiak I, Byers N, Evans K, Robinson T, et al. Multicentre randomized controlled trial of structured transition on diabetes care management compared to standard diabetes care in adolescents and young adults with type 1 diabetes (Transition Trial). BMC Pediatrics 2013;13:163.

\section{NCT01837121 \{published data only\}}

NCT01837121. A trial of using SMS reminder among diabetic retinopathy patients in rural China (SMS) [A randomized controlled trail to determine the impact of a SMS reminder among diabetic retinopathy patients in rural China]. clinicaltrials.gov/ct2/show/NCT01837121 (first submitted 14 April 2013).

\section{NCT02339909 \{published data only\}}

NCT02339909. Incentives in Diabetic Eye Assessment by Screening (IDEAS). clinicaltrials.gov/ct2/show/NCT02339909 (first submitted 15 December 2014).
NCT02579837 \{published data only\}

NCT02579837. CLEAR SIGHT: a trial of non-mydriatic ultrawidefield retinal imaging to screen for diabetic eye disease. clinicaltrials.gov/ct2/show/NCT02579837 (first submitted 16 October 2015).

\section{Additional references}

\section{American Diabetes Association 2015}

American Diabetes Association. Standards of medical care in diabetes-2015 abridged for primary care providers. Clinical Diabetes 2015;33(2):97-111.

\section{Byun 2013}

Byun SH, Ma SH, Jun JK, Jung KW, Park B. Screening for diabetic retinopathy and nephropathy in patients with diabetes: a nationwide survey in Korea. PloS One 2013;8(5):e62991.

\section{CDC 2012}

Centre for Reviews and Dissemination. Improving access to cost-effectiveness information for health care decision-making: the NHS Economic Evaluation Database (2nd Edition). CRD Report 6. 2001. www.york.ac.uk/crd/publications/reportsbooks/ (accessed 17 July 2017).

\section{EDTRS 1991}

Anonymous. Grading diabetic retinopathy from stereoscopic color fundus photographs--an extension of the modified Airlie House classification. ETDRS report number 10. Early Treatment Diabetic Retinopathy Study Research Group. Ophthalmology 1991;98(Suppl 5):786-806.

\section{EPOC 2012}

Cochrane Effective Practice and Organisation of Care Group (EPOC). Suggested risk of bias criteria for EPOC reviews. epoc.cochrane.org/sites/epoc.cochrane.org/files/uploads/ Suggested $\% 20$ risk $\% 20$ of $\% 20$ bias $\% 20$ criteria $\% 20$ for $\% 20$ EPOC \%20reviews.pdf (accessed 3 November 2015).

\section{EPOC 2015}

Cochrane Effective Practice and Organisation of Care Group (EPOC). EPOC Taxonomy, 2015. epoc.cochrane.org/epoctaxonomy (accessed 10 December 2015).

\section{EPOC 2017}

Cochrane Effective Practice, Organisation of Care (EPOC). Data collection form. EPOC Resources for review authors, 2017. epoc.cochrane.org/epoc-specific-resources-review-authors (accessed 20 August 2017).

\section{Evans 2014}

Evans JR, Michelessi M, Virgili G. Laser photocoagulation for proliferative diabetic retinopathy. Cochrane Database of Systematic Reviews 2014, Issue 11. [DOI: 10.1002/14651858.CD011234.pub2]

\section{Everett 2011}

Everett T, Bryant A, Griffin MF, Martin-Hirsch PP, Forbes CA, Jepson RG. Interventions targeted at women to encourage the 
uptake of cervical screening. Cochrane Database of Systematic Reviews 2011, Issue 5. [DOI: 10.1002/14651858.CD002834.pub2]

\section{Evers 2005}

Evers S, Goossens M, De Vet H, Van Tulder M, Ament A. Criteria list for assessment of methodological quality of economic evaluations: Consensus on Health Economic Criteria. International Journal of Technology Assessment in Health Care 2005;21(2):240-5.

\section{Fong 2004}

Fong DS, Aiello L, Gardner TW, King GL, Blankenship G, Cavallerano JD, et al. Retinopathy in diabetes. Diabetes Care 2004;27 Suppl 1:S84-7.

\section{Glanville 2006}

Glanville JM, Lefebvre C, Miles JN, Camosso-Stefinovic J. How to identify randomized controlled trials in MEDLINE: ten years on. Journal of the Medical Library Association 2006;94(2):1306.

\section{Grimshaw 2004}

Grimshaw JM, Thomas RE, MacLennan G, Fraser C, Ramsay CR, Vale $L$, et al. Effectiveness and efficiency of guideline dissemination and implementation strategies. Health Technology Assessment 2004;8(6):iii-iv, 1-72.

\section{Grimshaw 2014}

Grimshaw JM, Presseau J, Tetroe J, Eccles MP, Francis JJ, Godin G, et al. Looking inside the black box: results of a theorybased process evaluation exploring the results of a randomized controlled trial of printed educational messages to increase primary care physicians' diabetic retinopathy referrals [Trial registration number ISRCTN72772651]. Implementation Science : IS 2014;9:86.

\section{Gulliford 2010}

Gulliford MC, Dodhia H, Chamley M, McCormick K, Mohamed M, Naithani S, et al. Socio-economic and ethnic inequalities in diabetes retinal screening. Diabetic Medicine 2010;27(3):282-8.

\section{Heintz 2010}

Heintz E, Wirehn AB, Peebo BB, Rosenqvist U, Levin LA. Prevalence and healthcare costs of diabetic retinopathy: a population-based register study in Sweden. Diabetologia 2010;53(10):2147-54.

\section{Higgins 2011}

Higgins JP, Deeks JJ, Altman DG editor(s). Chapter 16: Special topics in statistics. In: Higgins JP, Green S, editor(s). Cochrane Handbook for Systematic Reviews of Interventions Version 5.1.0 (updated March 2011). The Cochrane Collaboration, 2011. Available from handbook.cochrane.org.

\section{Holden 2010}

Holden DJ, Jonas DE, Porterfield DS, Reuland D, Harris R. Systematic review: enhancing the use and quality of colorectal cancer screening. Annals of Internal Medicine 2010;152(10):668-76.

\section{Husereau 2013}

Husereau D, Drummond M, Petrou S, Carswell C, Moher D, Greenberg D, et al. Consolidated Health Economic Evaluation Reporting Standards (CHEERS)--explanation and elaboration: a report of the ISPOR Health Economic Evaluation Publication Guidelines Good Reporting Practices Task Force. Value in Health 2013;16(2):231-50.

\section{Hutchinson 2000}

Hutchinson A, McIntosh A, Peters J, O'Keeffe C, Khunti K, Baker $\mathrm{R}$, et al. Effectiveness of screening and monitoring tests for diabetic retinopathy--a systematic review. Diabetic Medicine 2000;17(7):495-506.

\section{Hwang 2015}

Hwang J, Rudnisky C, Bowen S, Johnson JA. Socioeconomic factors associated with visual impairment and ophthalmic care utilization in patients with type II diabetes. Canadian Journal of Ophthalmology 2015;50(2):119-26.

\section{Ivers 2014}

Ivers N, Tricco AC, Trikalinos TA, Dahabreh IJ, Danko KJ, Moher D, et al. Seeing the forests and the trees-innovative approaches to exploring heterogeneity in systematic reviews of complex interventions to enhance health system decisionmaking: a protocol. Systematic Reviews 2014;3:88.

\section{Jones 2010}

Jones S, Edwards RT. Diabetic retinopathy screening: a systematic review of the economic evidence. Diabetic Medicine 2010;27(3):249-56.

\section{Kirkizlar 2013}

Kirkizlar E, Serban N, Sisson JA, Swann JL, Barnes CS, Williams MD. Evaluation of telemedicine for screening of diabetic retinopathy in the Veterans Health Administration. Ophthalmology 2013;120(12):2604-10.

\section{Kliner 2012}

Kliner M, Fell G, Gibbons C, Dhothar M, Mookhtiar M, CasselsBrown A. Diabetic retinopathy equity profile in a multi-ethnic, deprived population in Northern England. Eye 2012;26(5):671-7.

\section{Kristinsson 1995}

Kristinsson JK, Gudmundsson JR, Stefansson E, Jonasson F, Gislason I, Thorsson AV. Screening for diabetic retinopathy. Initiation and frequency. Acta Ophthalmologica Scandinavica 1995;73(6):525-8.

\section{Lorencatto 2013}

Lorencatto F, West R, Stavri Z, Michie S. How well is intervention content described in published reports of smoking cessation interventions?. Nicotine \& Tobacco Research 2013;15(7):1273-82.

\section{Lund 2016}

Lund SH, Aspelund T, Kirby P, Russell G, Einarsson S, Palsson O, et al. Individualised risk assessment for diabetic retinopathy and optimisation of screening intervals: a scientific approach to reducing healthcare costs. British Journal of Ophthalmology 2016;100(5):683-7. 


\section{Michie 2009}

Michie S, Fixsen D, Grimshaw JM, Eccles MP. Specifying and reporting complex behaviour change interventions: the need for a scientific method. Implementation Science 2009; Vol. 4:40.

\section{Michie 2013}

Michie S, Richardson M, Johnston M, Abraham C, Francis J, Hardeman W, et al. The behavior change technique taxonomy (v1) of 93 hierarchically clustered techniques: building an international consensus for the reporting of behavior change interventions. Annals of Behavioral Medicine 2013;46(1):81-95.

\section{Millett 2006}

Millett C, Dodhia H. Diabetes retinopathy screening: audit of equity in participation and selected outcomes in South East London. Journal of Medical Screening 2006;13(3):152-5.

\section{O'Neill 2014}

O'Neill J, Tabish H, Welch V, Petticrew M, Pottie K, Clarke M, et al. Applying an equity lens to interventions: using PROGRESS ensures consideration of socially stratifying factors to illuminate inequities in health. Journal of Clinical Epidemiology 2014;67(1):56-64.

\section{Paz 2006}

Paz SH, Varma R, Klein R, Wu J, Azen SP. Noncompliance with vision care guidelines in Latinos with type 2 diabetes mellitus: the Los Angeles Latino Eye Study. Ophthalmology 2006;113(8):1372-7.

\section{Presseau 2015}

Presseau J, Ivers NM, Newham JJ, Knittle K, Danko KJ, Grimshaw JM. Using a behaviour change techniques taxonomy to identify active ingredients within trials of implementation interventions for diabetes care. Implementation Science 2015;10:55

\section{Review Manager 2014 [Computer program]}

Nordic Cochrane Centre. The Cochrane Collaboration. Review Manager 5 (RevMan 5). Version 5.3. Copenhagen: Nordic Cochrane Centre. The Cochrane Collaboration, 2014.

\section{Saadine 2008}

Saadine JB, Fong DS, Yao J. Factors associated with followup eye examinations among persons with diabetes. Retina 2008;28(2):195-200.

\section{Scanlon 2008}

Scanlon $\mathrm{PH}$. The English national screening programme for sight-threatening diabetic retinopathy. Journal of Medical Screening 2008;15(1):1-4.

\section{Sharp 2003}

Sharp PF, Olson J, Strachan F, Hipwell J, Ludbrook A, O'Donnell M, et al. The value of digital imaging in diabetic retinopathy. Health Technology Assessment 2003;7(30):1-119.

\section{Silva 2009}

Silva PS, Cavallerano JD, Aiello LM. Ocular telehealth initiatives in diabetic retinopathy. Current Diabetes Reports 2009;9(4):265-71.

\section{Sivaprasad 2012}

Sivaprasad S, Gupta B, Crosby-Nwaobi R, Evans J. Prevalence of diabetic retinopathy in various ethnic groups: a worldwide perspective. Survey of Ophthalmology 2012;57(4):347-70.

\section{Taylor 2007}

Taylor CR, Merin LM, Salunga AM, Hepworth JT, Crutcher TD, O'Day DM, et al. Improving diabetic retinopathy screening ratios using telemedicine-based digital retinal imaging technology: the Vine Hill study. Diabetes Care 2007;30(3):574-8.

\section{Terrin 2003}

Terrin N, Schmid CH, Lau J, Olkin I. Adjusting for publication bias in the presence of heterogeneity. Statistics in Medicine 2003;22(13):2113-26.

\section{Tricco 2012}

Tricco AC, Ivers NM, Grimshaw JM, Moher D, Turner L, Galipeau J, et al. Effectiveness of quality improvement strategies on the management of diabetes: a systematic review and meta-analysis. Lancet 2012;379(9833):2252-61.

\section{Virgili 2014}

Virgili G, Parravano M, Menchini F, Evans JR. Anti-vascular endothelial growth factor for diabetic macular oedema. Cochrane Database of Systematic Reviews 2014, Issue 10. [DOI: 10.1002/14651858.CD007419.pub4]

\section{Waqar 2012}

Waqar S, Bullen G, Chant S, Salman R, Vaidya B, Ling R. Cost implications, deprivation and geodemographic segmentation analysis of non-attenders (DNA) in an established diabetic retinopathy screening programme. Diabetes and Metabolic Syndrome 2012;6(4):199-202.

\section{Williams 2004}

Williams GA, Scott IU, Haller JA, Maguire AM, Marcus D, McDonald HR. Single-field fundus photography for diabetic retinopathy screening: a report by the American Academy of Ophthalmology. Ophthalmology 2004;111(5):1055-62.

\section{Worswick 2013}

Worswick J, Wayne SC, Bennett R, Fiander M, Mayhew A, Weir MC, et al. Improving quality of care for persons with diabetes: an overview of systematic reviews - what does the evidence tell us?. Systematic Reviews 2013;2:26.

\section{Yau 2012}

Yau JW, Rogers SL, Kawasaki R, Lamoureux EL, Kowalski JW, Bek T, et al. Global prevalence and major risk factors of diabetic retinopathy. Diabetes Care 2012;35(3):556-64.

\section{Zhang 2007}

Zhang X, Norris SL, Saadine J, Chowdhury FM, Horsley T, Kanjilal S, et al. Effectiveness of interventions to promote screening for diabetic retinopathy. American Journal of Preventive Medicine 2007;33(4):318-35. 


\section{References to other published versions of this review}

\section{Lawrenson 2016}

Lawrenson JG, Graham-Rowe E, Lorencatto F, Presseau J, Burr J, Ivers N, et al. Interventions to increase attendance for diabetic retinopathy screening. Cochrane

\section{CHARACTERISTICS OF STUDIES}

Characteristics of included studies [ordered by study ID]
Database of Systematic Reviews 2016, Issue 1. [DOI: 10.1002/14651858.CD012054]

* Indicates the major publication for the study

Adair 2013

Methods Study aim: to test whether patients with chronic disease working with lay "care guides" would achieve more evidence-based goals than those receiving usual care

Study design: parallel-group RCT

Country: USA
Setting: Six primary care clinics in Minnesota
Total number of participants: 2135 patients with hypertension, diabetes or congestive heart failure
(1366 with diabetes)

Percentage male: $51 \%$

Diabetes type: type 1 and 2

Average age (SD): 60.5 yrs (11.5)

Inclusion criteria: age 18 - 79 yrs and with a primary care office visit during the 6-month enrolment period

Exclusion criteria: pregnancy

Intervention ( $\mathbf{n}=\mathbf{9 3 0}$ ): participants provided with disease-specific care goals and culturally-matched laypersons acting as 'care guides' helped participants to achieve goals. Care guides met with participants in person and/or were contacted by telephone

Comparator ( $\mathbf{n}=\mathbf{4 3 6}$ ): participants were provided with care goals followed by usual clinical care

Duration: 12 months

Outcomes

Primary outcome: change in the $\%$ of disease-specific care goals met 12 months after enrolment compared to baseline

Secondary outcomes: percentage of goals met by participants with each diagnosis and the achievement of each individual goal determined from electronic patient records (included 'retinal examination within 2yrs'); to determine whether the benefit of working with the care guide could be predicted by participant demographics

Baseline screening attendance (control group): $60.6 \%$

Trial registration number: NCT01156974

Sources of funding: Robina Foundation

Declaration of interest: none declared (Quote "Disclosures can be viewed at https://www.acponline.org/authors/icmje/ConflictOfInterestForms.do?msNum=M12-3106") 


\section{Risk of bias}

\begin{tabular}{|c|c|c|}
\hline Bias & Authors' judgement & Support for judgement \\
\hline $\begin{array}{l}\text { Adequate sequence gener- } \\
\text { ation? }\end{array}$ & Low risk & $\begin{array}{l}\text { Quote "Research supervisors prepared sealed opaque envelopes containing } \\
\text { either a purple card (assignment to a care guide) or gold card (assignment } \\
\text { to usual care). One hundred eighty envelopes ( } 120 \text { with purple cards and } 60 \\
\text { with gold cards) were given to the small clinic, } 360 \text { ( } 240 \text { purple and } 120 \text { gold } \\
\text { cards) were given to the medium-sized clinics, and } 540 \text { ( } 360 \text { purple and } 180 \\
\text { gold cards) were given to the large clinic. Each clinic's envelopes were shuffled } \\
\text { before delivery and daily thereafter." p } 177\end{array}$ \\
\hline
\end{tabular}

\begin{tabular}{ll}
\hline $\begin{array}{l}\text { Adequate allocation con- } \\
\text { cealement? }\end{array}$ & Quote "Research supervisors prepared sealed opaque envelopes...' \\
& $\begin{array}{l}\text { Quote 'Patients who consented to enroll received identical written informa- } \\
\text { tion about the benefits of meeting disease-specific goals. They then selected } \\
\text { and opened an envelope to determine treatment assignment." p } 177\end{array}$
\end{tabular}

\begin{tabular}{|c|c|c|}
\hline $\begin{array}{l}\text { Similar baseline outcome } \\
\text { measurements? }\end{array}$ & Low risk & $\begin{array}{l}\text { Judgement comment: similar baseline retinopathy screening attendance be- } \\
\text { tween arms. Table } 3 \text { p } 179\end{array}$ \\
\hline $\begin{array}{l}\text { Similar baseline character- } \\
\text { istics? }\end{array}$ & Low risk & Judgement comment: similar baseline characteristics. Table 2 p 179 \\
\hline $\begin{array}{l}\text { Incomplete outcome data } \\
\text { addressed? }\end{array}$ & Low risk & $\begin{array}{l}\text { Judgement comment: low attrition and missing data balanced across both } \\
\text { arms of the trial }\end{array}$ \\
\hline \multirow[t]{2}{*}{$\begin{array}{l}\text { Knowledge of allocated in- } \\
\text { tervention prevented? }\end{array}$} & High risk & $\begin{array}{l}\text { Quote "Patients, providers, and persons performing outcome assessments } \\
\text { were not blinded to treatment assignment." p } 176\end{array}$ \\
\hline & & $\begin{array}{l}\text { Judgement comment: retinopathy screening data extracted from electronic } \\
\text { patient record and knowledge of allocation could have influenced outcome }\end{array}$ \\
\hline $\begin{array}{l}\text { Protected against contam- } \\
\text { ination? }\end{array}$ & Low risk & $\begin{array}{l}\text { Quote: "Care guides and the research team did not interact with the usual care } \\
\text { patients after enrollment and randomization." p } 178\end{array}$ \\
\hline $\begin{array}{l}\text { Free from selective out- } \\
\text { come reporting? }\end{array}$ & Low risk & $\begin{array}{l}\text { Judgement comment: reported outcomes consistent with trial registry } \\
\text { NCT01156974 }\end{array}$ \\
\hline Other risks of bias? & Low risk & Judgement comment: no evidence of other sources of bias \\
\hline
\end{tabular}

Anderson 2003

\begin{tabular}{ll}
\hline Study aim: to evaluate the effectiveness of personalised follow-up compared to reminder letters, in \\
increasing return rates at urban eye disease screening clinics for African Americans with diabetes and \\
minimal or no retinopathy
\end{tabular}

Study design: parallel-group RCT

\section{Participants}

\section{Country: USA}

Setting: 9 free culture-specific (urban African American) community-based eye screening clinics

Total number of participants: 132 
Anderson 2003 (Continued)

Percentage male: $38 \%$

Diabetes type: type 2

Average age (SD): 55 yrs (NR)

Inclusion criteria: African-American adults with type 2 diabetes attending community eye clinic

Exclusion criteria: patients who were not African American

Interventions

Intervention ( $\mathbf{n}=\mathbf{6 7}$ ): single reminder letter including information on the day, time and location of the eye clinic appointment 1 month prior to the appointment. Follow-up phone call 10 days after letter sent. Phone call also addressed barriers to attending and message that diabetes can lead to vision loss

Comparator ( $\mathbf{n}=\mathbf{6 5}$ ): single reminder letter including information on the day, time and location of the eye clinic appointment 1 month prior to the appointment

Duration: 12 months

\begin{tabular}{ll} 
Outcomes & Primary outcome: return rate for annual dilated fundus examination \\
& Secondary outcomes: factors predicative of returning for a dilated fundus examination \\
& Baseline screening attendance (control group): $26.2 \%$ \\
\hline Notes & Date conducted: 1995 to 1999 \\
& Trial registration number: NR \\
& $\begin{array}{l}\text { Sources of funding: National Institute of Health/National Institute of Diabetes and Digestive and Kid- } \\
\text { ney Disease }\end{array}$
\end{tabular}

Declaration of interest: NR

\section{Risk of bias}

\begin{tabular}{|c|c|c|}
\hline Bias & Authors' judgement & Support for judgement \\
\hline $\begin{array}{l}\text { Adequate sequence gener- } \\
\text { ation? }\end{array}$ & Unclear risk & Not reported \\
\hline $\begin{array}{l}\text { Adequate allocation con- } \\
\text { cealement? }\end{array}$ & Unclear risk & Not reported \\
\hline $\begin{array}{l}\text { Similar baseline outcome } \\
\text { measurements? }\end{array}$ & Low risk & $\begin{array}{l}\text { Judgement comment: similar numbers of participants in each arm having ever } \\
\text { had an eye examination by an ophthalmologist with similar numbers screened } \\
\text { in last year (see Table } 1 \text { p 43) }\end{array}$ \\
\hline $\begin{array}{l}\text { Similar baseline character- } \\
\text { istics? }\end{array}$ & Low risk & $\begin{array}{l}\text { Quote "There were no statistically significant differences between the } 2 \text { groups } \\
\text { on any of the variables in this table." (Footnote Table } 1 \mathrm{p} \mathrm{43)}\end{array}$ \\
\hline $\begin{array}{l}\text { Incomplete outcome data } \\
\text { addressed? }\end{array}$ & Low risk & Judgement comment: all outcome data reported. See Table 1 p 42 \\
\hline $\begin{array}{l}\text { Knowledge of allocated in- } \\
\text { tervention prevented? }\end{array}$ & Unclear risk & Not reported \\
\hline $\begin{array}{l}\text { Protected against contam- } \\
\text { ination? }\end{array}$ & Low risk & $\begin{array}{l}\text { Judgement comment: it is unlikely that the control group received the tele- } \\
\text { phone reminder }\end{array}$ \\
\hline
\end{tabular}


Anderson 2003 (Continued)

Free from selective out- Unclear risk Judgement comment: no protocol or trial registry entry available and therecome reporting? fore not possible to assess

Other risks of bias?

Low risk

Judgement comment: no evidence of other risks of bias

Barcelo 2010

Methods Study aim: to assess the impact of integrated care, comprising specialist support, collaborative learning and case management, on the quality of diabetes care

Study design: cluster-RCT

Participants Country: Mexico

Setting: 10 urban public health centres

Number of clusters: 10

Number of providers: 43 primary care teams

Total number of patients: 307

Percentage male: NR

Diabetes type: type 1 and 2 (97.4\% type 2)

Average age (SD): NR

Inclusion criteria: participants were selected based on "their capacity to communicate, their advanced knowledge of diabetes, and their willingness to collaborate"

Exclusion criteria: NR

Interventions

Intervention (5 clusters, $\mathbf{n}=\mathbf{1 9 6}$ ): diabetes education programme, in-service training of primary care personnel. specialist support to primary care, case management of participants not achieving care goals

Comparator (5 clusters, $\mathbf{n}=\mathbf{1 1 1}$ ): usual care (not specified)

Duration: 3 learning sessions within 18 months

Outcomes

Primary outcome: change in the proportion of participants achieving quality improvement targets (metabolic control, cholesterol, blood pressure, eye and foot examinations)

\section{Secondary outcomes: NR}

Baseline screening attendance (control group): $3.6 \%$

Notes

Date conducted: November 2002 to May 2004

Trial registration number: NR

Sources of funding: NR

Declaration of interest: none declared

\section{Risk of bias}


Barcelo 2010 (Continued)

Adequate sequence gener- Unclear risk Not reported
ation?

Adequate allocation con- Low risk cealement?

Similar baseline outcome Low risk
measurements?
measurements?
Judgement comment: unit of allocation by community health centre and allocation performed prior to the start of the study

\begin{tabular}{|c|c|c|}
\hline $\begin{array}{l}\text { Similar baseline character- } \\
\text { istics? }\end{array}$ & Low risk & $\begin{array}{l}\text { Judgement comment: baseline characteristics of participants were similar in } \\
\text { each arm (seeTable } 1 \text { and } 2 \text { p } 148 \text {-9) }\end{array}$ \\
\hline $\begin{array}{l}\text { Incomplete outcome data } \\
\text { addressed? }\end{array}$ & Unclear risk & $\begin{array}{l}\text { Judgement comment: cannot tell whether an ITT or per-protocol analysis was } \\
\text { conducted. No flow diagram provided with losses to follow-up, do not know } \\
\text { whether losses to follow-up were similar between both arms }\end{array}$ \\
\hline $\begin{array}{l}\text { Knowledge of allocated in- } \\
\text { tervention prevented? }\end{array}$ & Unclear risk & Not reported \\
\hline $\begin{array}{l}\text { Protected against contam- } \\
\text { ination? }\end{array}$ & High risk & $\begin{array}{l}\text { Quote: "... avoiding the "contamination" of centers that acted as controls } \\
\text { (those centers providing usual diabetes care) was not possible, because of the } \\
\text { visibility and publicity of the intervention at the local level." p } 151\end{array}$ \\
\hline $\begin{array}{l}\text { Free from selective out- } \\
\text { come reporting? }\end{array}$ & Unclear risk & $\begin{array}{l}\text { Judgement comment: no protocol or trial registry entry available and there- } \\
\text { fore not possible to assess }\end{array}$ \\
\hline Other risks of bias? & Low risk & Judgement comment: no evidence of other sources of bias \\
\hline
\end{tabular}

\section{Basch 1999}

Study aim: to evaluate the impact of a multicomponent health education intervention on the rate of
ophthalmic examinations in African Americans with diabetes

Study design: parallel-group RCT

\begin{tabular}{ll}
\hline Participants & Country: USA \\
& Setting: outpatient clinics at 5 sites in the New York metropolitan area with on-site ophthalmology ser- \\
vices (secondary care)
\end{tabular}

\section{Total number of participants: 280}

Percentage male: $34.3 \%$

Diabetes type: NR

Average age (SD): 54.8 yrs (12.9)

Inclusion criteria: African-American patients $>18 \mathrm{yrs}$ with a diagnosis of diabetes with no record of receiving a dilated eye exam in the preceding 14 months

Exclusion criteria: blindness in both eyes, advanced eye disease, progressive medical illness, impaired cognitive ability 
Comparator $(\mathbf{n}=\mathbf{1 4 3})$ : mailed booklet produced by the American Medical Association on meal planning

Duration: 6 months (or until eye exam recorded)

\begin{tabular}{ll}
\hline Outcomes & Primary outcome: documented dilated retinal examination within 6 months of randomisation \\
& Secondary outcomes: predictors of examination status \\
& Baseline screening attendance (control group): $0 \%$ \\
\hline Notes & Date conducted: 1993 to 1995 \\
& Trial registration number: NR \\
& $\begin{array}{l}\text { Sources of funding: National Eye Institute, National Institute of Diabetes and Digestive and Kidney Dis- } \\
\text { ease }\end{array}$ \\
& Declaration of interest: none declared
\end{tabular}

\section{Risk of bias}

\begin{tabular}{lll}
\hline Bias & Authors' judgement & Support for judgement \\
\hline $\begin{array}{ll}\text { Adequate sequence gener- } \\
\text { ation? }\end{array}$ & Low risk & $\begin{array}{l}\text { Quote "After research staff confirmed subjects could be reached by telephone, } \\
\text { they were enrolled and randomised within site and sex groups. We random- } \\
\text { ized subjects in pairs by using tables of random permutations." } \mathrm{p} 1879\end{array}$
\end{tabular}

\begin{tabular}{lll}
\hline $\begin{array}{l}\text { Adequate allocation con- } \\
\text { cealement? }\end{array}$ & Unclear risk & Not reported \\
\hline $\begin{array}{l}\text { Similar baseline outcome } \\
\text { measurements? }\end{array}$ & Low risk & $\begin{array}{l}\text { Quote: "Eligibility criteria based on chart audits included a diagnosis of dia- } \\
\text { betes mellitus, being African American, being } 18 \text { years or older, having no doc- } \\
\text { umentation of a dilated retinal examination in the preceding } 14 \text { months, and } \\
\text { having been seen at the clinic at least } 1 \text { other time in the past year." } \mathrm{p} 1879\end{array}$
\end{tabular}

\begin{tabular}{|c|c|c|}
\hline $\begin{array}{l}\text { Similar baseline character- } \\
\text { istics? }\end{array}$ & Low risk & $\begin{array}{l}\text { Quote "There were no significant differences between groups on any of the } \\
\text { available personal and demographic variables" (see Table } 1 \text { p 1880) }\end{array}$ \\
\hline $\begin{array}{l}\text { Knowledge of allocated in- } \\
\text { tervention prevented? }\end{array}$ & Low risk & $\begin{array}{l}\text { Quote "Research staff, unaware of subjects' group assignment, audited med- } \\
\text { ical records." p } 1879\end{array}$ \\
\hline $\begin{array}{l}\text { Protected against contam- } \\
\text { ination? }\end{array}$ & Low risk & $\begin{array}{l}\text { Judgement comment: it is unlikely that the control group received the multi- } \\
\text { component health education intervention }\end{array}$ \\
\hline $\begin{array}{l}\text { Free from selective out- } \\
\text { come reporting? }\end{array}$ & Unclear risk & $\begin{array}{l}\text { Judgement comment: no protocol or trial registry entry available and there- } \\
\text { fore not possible to assess }\end{array}$ \\
\hline Other risks of bias? & Low risk & Judgement comment: no evidence of other risks of bias \\
\hline
\end{tabular}

Bush 2014

Methods

Study aim: to evaluate the impact of 'Link Workers' on the uptake of diabetic retinopathy screening in a hard-to-reach and high-risk population group 
Bush 2014 (Continued)

Study design: cluster-RCT

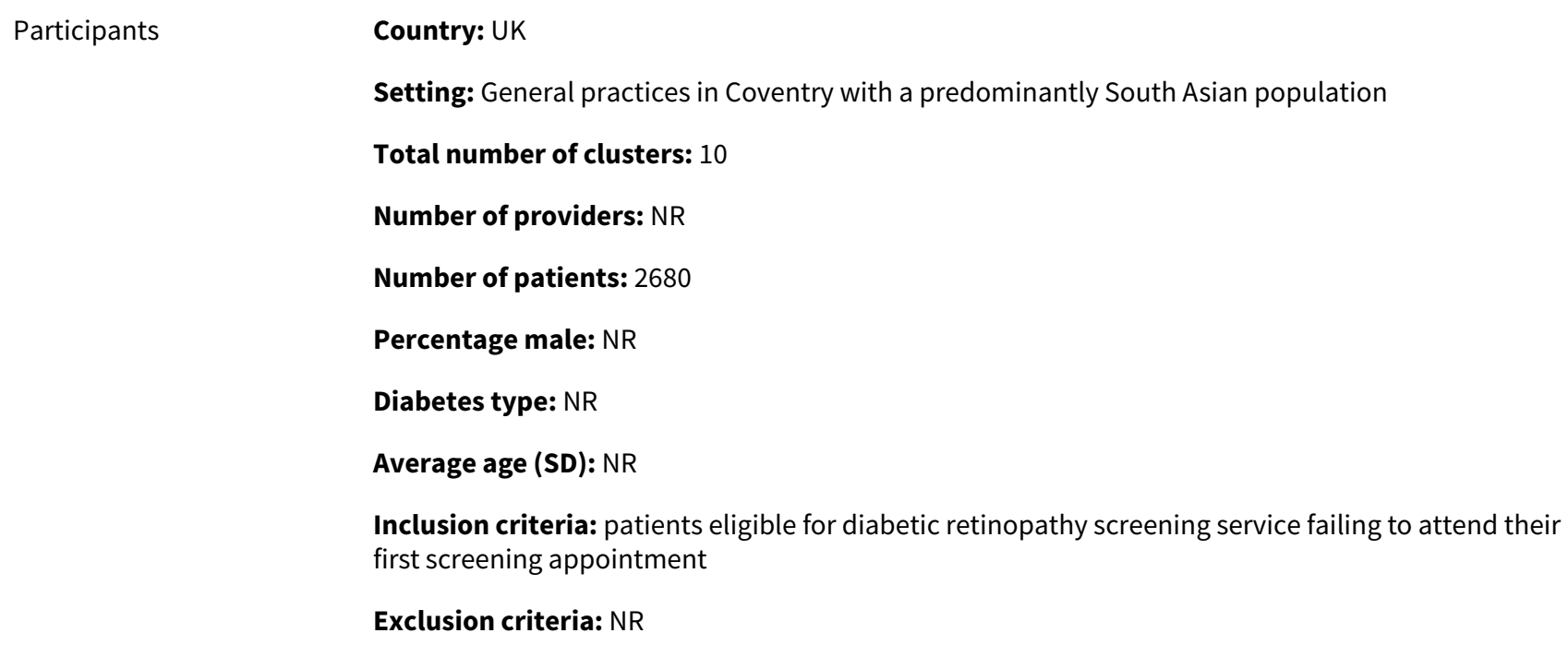

Exclusion criteria: NR ipants failing to attend their first appointment to remind them of the screening appointment and encourage attendance

Comparator (5 clusters, $\mathbf{n}=\mathbf{1 6 9 2}$ participants): usual care (participants who failed to attend their initial screen date were sent a further appointment date by post).

Duration: phone calls continued until an examination was reported or after 6 months, whichever came first

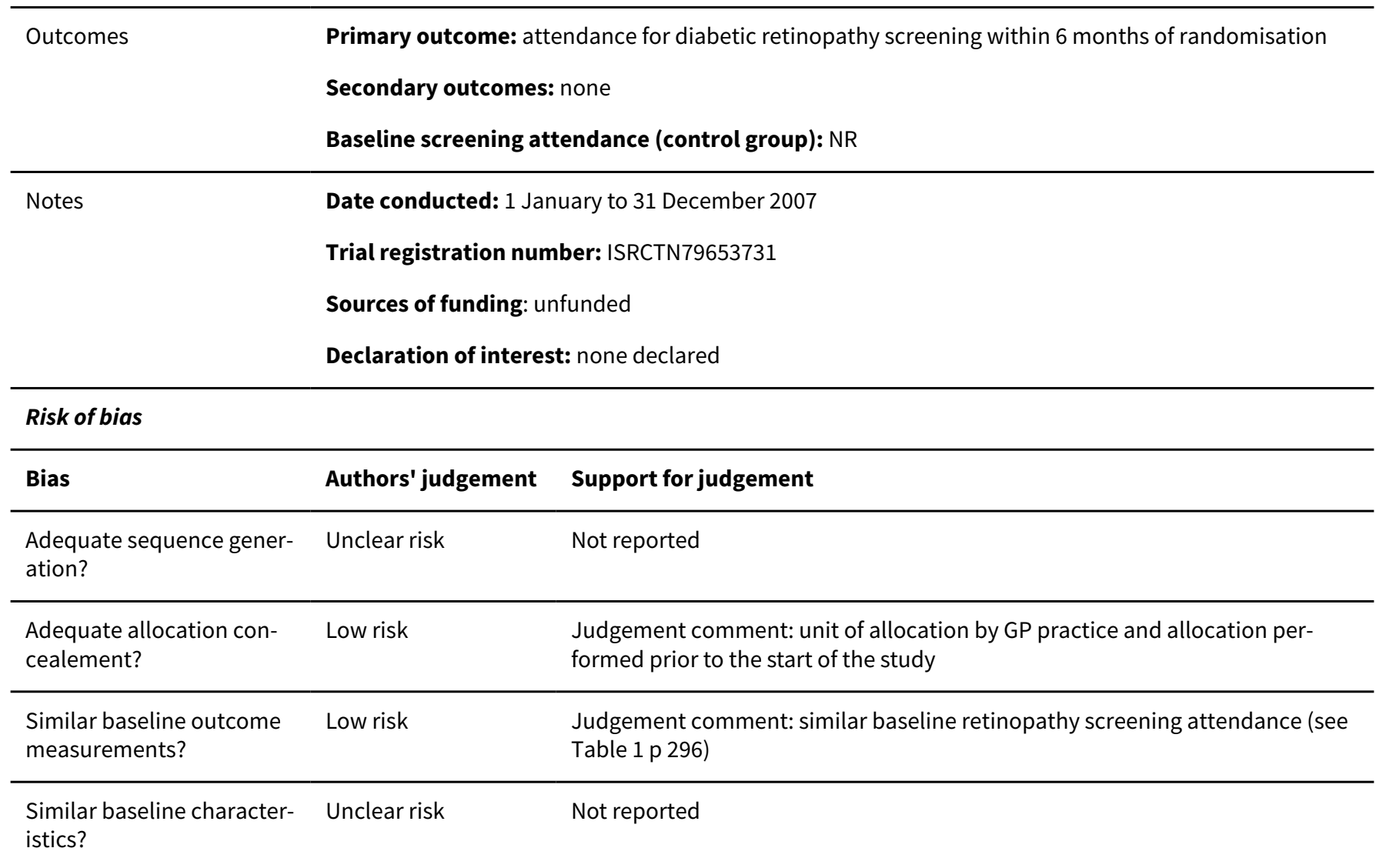


Bush 2014 (Continued)

Incomplete outcome data Low risk Judgement comment: data reported for all participants
addressed?

\begin{tabular}{lll}
$\begin{array}{l}\text { Knowledge of allocated in- } \\
\text { tervention prevented? }\end{array}$ & Low risk & $\begin{array}{l}\text { Quote "Data available for analyses comprised routinely collected and collated } \\
\text { attendance data from the retinopathy screening unit." p 295 }\end{array}$ \\
\hline $\begin{array}{l}\text { Protected against contam- } \\
\text { ination? }\end{array}$ & Low risk & $\begin{array}{l}\text { Quote "Following randomisation and throughout the study, there was no fur- } \\
\text { ther contact with control practices." p } 295\end{array}$ \\
\hline $\begin{array}{l}\text { Free from selective out- } \\
\text { come reporting? }\end{array}$ & Unclear risk & $\begin{array}{l}\text { Judgement comment: trial retrospectively registered and so not possible to } \\
\text { assess }\end{array}$ \\
\hline \begin{tabular}{ll} 
Other risks of bias? \\
\hline
\end{tabular} & Low risk & Judgement comment: no evidence of other risks of bias \\
\hline
\end{tabular}

\section{Choe 2005}

Methods Study aim: to evaluate the effect of case management by a clinical pharmacist on glycaemic control and preventive measures in patients with type 2 diabetes mellitus

Study design: parallel-group RCT

Country: USA
Setting: university-affiliated primary care internal medicine clinic
Total number of participants: 80
Percentage male: $47.5 \%$
Diabetes type: type 2
Average age (SD): 51.6 yrs (10.1)
Inclusion criteria: high-risk individuals whose most recent HbA1c levels $\geq 8.0 \%$
Exclusion criteria: type 1 diabetes mellitus (based on diagnosis before age 30 years), if they were $>70$
years, or if they were diagnosed as having cancer, renal failure, severe cirrhosis, malignant hyperten-
sion, or a severe concurrent illness that would substantially limit life expectancy or require extensive
systemic treatment

Interventions

Intervention ( $\mathbf{n = 4 1 ) : ~ o n - s i t e ~ c l i n i c a l ~ p h a r m a c i s t ~ a c t i n g ~ a s ~ a ~ c a s e ~ m a n a g e r , ~ p r o v i d i n g ~ e v a l u a t i o n ~ a n d ~}$ modification of pharmacotherapy, self-management diabetes education (including an emphasis on the importance of self-care, medications, and screening processes). Generally, the clinical pharmacist contacted the participants by telephone on a monthly basis, unless more frequent assessment or recommendations were needed, and saw the participants in conjunction with routine primary care visits

Comparator $(\mathbf{n}=\mathbf{3 9})$ : usual care (unspecified)

Duration: 12 months

Outcomes Primary outcome: HbA1c level at 12 months

Secondary outcomes: diabetes process measures, including low-density lipoprotein measurement, dilated retinal examination, urine microalbumin screening (or use of angiotensin-converting enzyme inhibitors), and monofilament testing for diabetic neuropathy within the 2-year time frame of the study

Baseline screening attendance (control group): NR

Notes Date conducted: NR


Choe 2005 (Continued)

\title{
Trial registration number: NR
}

Sources of funding: funding for the clinical pharmacist was provided by the University of Michigan College of Pharmacy

Declaration of interest: NR

\section{Risk of bias}

\begin{tabular}{|c|c|c|}
\hline Bias & Authors' judgement & Support for judgement \\
\hline \multirow{3}{*}{$\begin{array}{l}\text { Adequate sequence gener- } \\
\text { ation? }\end{array}$} & Low risk & Quote: "Randomization within each stratum was simple: because \\
\hline & & $\begin{array}{l}\text { the study was small, randomization was done by hand,drawing numbers from } \\
\text { a container that included "0" for the control group or "1" for the intervention } \\
\text { group." }\end{array}$ \\
\hline & & p 255 \\
\hline $\begin{array}{l}\text { Adequate allocation con- } \\
\text { cealement? }\end{array}$ & Unclear risk & Not reported \\
\hline $\begin{array}{l}\text { Similar baseline outcome } \\
\text { measurements? }\end{array}$ & Unclear risk & Not reported \\
\hline $\begin{array}{l}\text { Similar baseline character- } \\
\text { istics? }\end{array}$ & Low risk & $\begin{array}{l}\text { Judgement comment: baseline characteristics of participants were similar in } \\
\text { each arm (see Table } 1 \text { p 256) }\end{array}$ \\
\hline $\begin{array}{l}\text { Incomplete outcome data } \\
\text { addressed? }\end{array}$ & Low risk & $\begin{array}{l}\text { Judgement comment: attrition not balanced across arms ( } 12 \% \text { loss to fol- } \\
\text { low-up in intervention group and } 26 \% \text { in control group). See CONSORT flow di- } \\
\text { agram p } 255\end{array}$ \\
\hline $\begin{array}{l}\text { Knowledge of allocated in- } \\
\text { tervention prevented? }\end{array}$ & Low risk & $\begin{array}{l}\text { Judgement comment: data on eye screening obtained by chart review but not } \\
\text { clear if outcome assessor was masked }\end{array}$ \\
\hline $\begin{array}{l}\text { Protected against contam- } \\
\text { ination? }\end{array}$ & Unclear risk & $\begin{array}{l}\text { Judgement comment: control group not described and not clear if contamina- } \\
\text { tion was prevented }\end{array}$ \\
\hline $\begin{array}{l}\text { Free from selective out- } \\
\text { come reporting? }\end{array}$ & Unclear risk & $\begin{array}{l}\text { Judgement comment: no protocol or trial registry entry available and there- } \\
\text { fore not possible to assess }\end{array}$ \\
\hline Other risks of bias? & Low risk & Judgement comment: no evidence of other sources of bias \\
\hline
\end{tabular}

Clancy 2007

Methods

Study aim: to evaluate the effect of group visits on clinical outcomes concordant with 10 American Diabetes Association (ADA) guideline processes of care

Study design: parallel-group RCT

Participants

\author{
Country: USA \\ Setting: adult primary care centre, Medical University of South Carolina
}

Total number of participants: 186

Percentage male: $28 \%$

Diabetes type: type 2 
Clancy 2007 (Continued)

$$
\text { Average age (SD): } 56 \text { yrs (NR) }
$$

Inclusion criteria: aged $>18$ years with poorly-controlled diabetes mellitus ( $\mathrm{HbA1c}>8.0 \%)$

Exclusion criteria: primary diagnosis of substance abuse or dependence; current pregnancy; dementia; inability to hear, speak English; obtain transportation to the clinic

Interventions

Intervention ( $\mathbf{n}=96)$ : monthly group visits (14 - 17 per group), co-led by an internal medicine physician and a registered nurse. One-on-one visits were available for care as needed between scheduled group visits or for specific medical needs not amenable to group visits. Group visit content consisted of educational topics such as nutrition, exercise, foot care, medications, complications of diabetes, and the emotional aspects of diabetes

Comparator $(\mathbf{n}=\mathbf{9 0})$ : control participants received usual care in the clinic, seeing faculty or resident physicians, physician assistants, nurse practitioners, or medical or physician assistant students with access to a dietician and diabetes educator

Duration: 12 months

\section{Outcomes}

Primary outcome: 10 ADA process-of-care indicators ( > 2 yearly HgAlc, at least yearly cholesterol levels, treatment for LDL cholesterol levels $>100 \mathrm{mg} / \mathrm{dl}$, yearly ophthalmologic referrals, influenza vaccinations, foot exams, and checks for microalbuminuria, ACE-inhibitor or angiotensin receptor blocker use, daily aspirin unless contraindicated, and at least 1 pneumococcal vaccine)

\section{Secondary outcomes: NR}

Baseline screening attendance (control group): NR

Notes Date conducted: September 2002 to February 2003

Trial registration number: NR

Sources of funding: Agency for Healthcare Research and Quality; Robert Wood Johnson Foundation; National Institutes of Health

Declaration of interest: 2 authors reported receiving grants from Pfizer and Elli Lilly

\section{Risk of bias}

\begin{tabular}{|c|c|c|}
\hline Bias & Authors' judgement & Support for judgement \\
\hline $\begin{array}{l}\text { Adequate sequence gener- } \\
\text { ation? }\end{array}$ & Low risk & $\begin{array}{l}\text { Quote: "Subjects meeting criteria for inclusion into the study were randomized } \\
\text { after informed consent and baseline data collection using randlst software } \\
\text { (http://odin.mdacc.tmc.edu/anonftp/) allowing for stratification and blocking. } \\
\text { Subjects were stratified by race and gender using a block size of } 4 . " \text { p } 621\end{array}$ \\
\hline $\begin{array}{l}\text { Adequate allocation con- } \\
\text { cealement? }\end{array}$ & Unclear risk & Not reported \\
\hline $\begin{array}{l}\text { Similar baseline outcome } \\
\text { measurements? }\end{array}$ & Unclear risk & Not reported \\
\hline \multirow[t]{2}{*}{$\begin{array}{l}\text { Similar baseline character- } \\
\text { istics? }\end{array}$} & Low risk & $\begin{array}{l}\text { Quote: "Demographic variables were well balanced between patients random- } \\
\text { ized to group visits or usual care at baseline (Table 1)." p } 622\end{array}$ \\
\hline & & $\begin{array}{l}\text { Quote: "Clinical variables were also well balanced at baseline (Table 1) 'with } \\
\text { a mean HgbA1c level at baseline of } 9.3 \% \text { for group patients and } 8.9 \% \text { for con- } \\
\text { trol patients. The mean total cholesterol level for group patients was } 193.4 \text { and } \\
196.1 \mathrm{mg} / \mathrm{dl} \text { for control patients. Blood pressures, triglycerides, LDL, and HDL } \\
\text { levels showed no significant baseline differences between the } 2 \text { groups." p } 622\end{array}$ \\
\hline
\end{tabular}


Clancy 2007 (Continued)

Incomplete outcome data Low risk Judgement comment: missing data balanced across 2 arms of study (17\% in addressed? the intervention arm and $16 \%$ in the comparator arm). Reasons given for missing data

Knowledge of allocated in- Low risk tervention prevented?

Quote: "Upon study completion, medical records were blindly abstracted for the 10 ADA process-of-care indicators." p 621

Protected against contam- High risk ination?

Quote: "These providers also had patients in the usual care arm as part of the general pool of clinic patients; thus, it is possible through contamination that providers may have adopted some of the group visit strategies (e.g., group visit educational content) for control patients." p 623

\begin{tabular}{lll}
\hline $\begin{array}{l}\text { Free from selective out- } \\
\text { come reporting? }\end{array}$ & Unclear risk & $\begin{array}{l}\text { Judgement comment: no protocol or trial registry entry available and there- } \\
\text { fore not possible to assess }\end{array}$ \\
\hline Other risks of bias? & Low risk & Judgement comment: no evidence of other sources of bias \\
\hline
\end{tabular}

\section{Conlin 2006}

Methods

Study aim: to study whether non-mydriatic digital retinal imaging in an ambulatory care setting affected adherence to annual dilated ophthalmic examinations in patients with diabetes

Study design: parallel-group RCT

\section{Participants}

\section{Country: USA}

Setting: Department of Veterans Affairs (VA) Boston Healthcare System

Total number of participants: 448

Percentage male: $98 \%$

Diabetes type: NR

Average age (SD): 67 yrs (21.2)

Inclusion criteria: adults with diabetes and a VA-based primary care provider

Exclusion criteria: NR

Interventions

Intervention ( $\mathbf{n}=\mathbf{2 2 3}$ ): teleretinal imaging by trained imager who demonstrated to the participant using the retinal images, the basic anatomical structures of the ocular fundus. Acting as a care co-ordinator, the imager later acted on the image reader's report when necessary and communicated with the participant to establish an appropriate eye-exam schedule. The imager also educated the participant about the importance of optimal blood glucose and blood pressure control

Comparator ( $\mathbf{n}=\mathbf{2 2 5})$ : usual care (not specified)

Duration: 12 months

Outcomes

Primary outcome: documented dilated retinal examination within 12 months of randomisation

Secondary outcomes: diabetic retinopathy outcomes and characteristics of participants with ungradable images

Baseline screening attendance (control group): NR 
Conlin 2006 (Continued)

\section{Trial registration number: NR}

Sources of funding: Department of the Army; VA Health Services Research and Development Service; National Institutes of Health

Declaration of interest: none declared

\begin{tabular}{|c|c|c|}
\hline \multicolumn{3}{|l|}{ Risk of bias } \\
\hline Bias & Authors' judgement & Support for judgement \\
\hline $\begin{array}{l}\text { Adequate sequence gener- } \\
\text { ation? }\end{array}$ & Low risk & $\begin{array}{l}\text { Quote: "Randomization was accomplished with a random-variables generator } \\
\text { and a series of sealed envelopes." p } 734\end{array}$ \\
\hline $\begin{array}{l}\text { Adequate allocation con- } \\
\text { cealement? }\end{array}$ & Unclear risk & $\begin{array}{l}\text { Quote: "Randomization was accomplished with a random-variables generator } \\
\text { and a series of sealed envelopes." p } 734\end{array}$ \\
\hline & & $\begin{array}{l}\text { Judgment comment: not clear whether the envelope was assigned to the par- } \\
\text { ticipant before opening }\end{array}$ \\
\hline $\begin{array}{l}\text { Similar baseline outcome } \\
\text { measurements? }\end{array}$ & Unclear risk & Not reported \\
\hline $\begin{array}{l}\text { Similar baseline character- } \\
\text { istics? }\end{array}$ & Unclear risk & Not reported \\
\hline $\begin{array}{l}\text { Incomplete outcome data } \\
\text { addressed? }\end{array}$ & Low risk & Judgement comment: data available for all participants (see Table 2) \\
\hline $\begin{array}{l}\text { Knowledge of allocated in- } \\
\text { tervention prevented? }\end{array}$ & Unclear risk & Not reported \\
\hline $\begin{array}{l}\text { Protected against contam- } \\
\text { ination? }\end{array}$ & Low risk & $\begin{array}{l}\text { Judgement comment: it is unlikely that the control group received teleretinal } \\
\text { imaging }\end{array}$ \\
\hline $\begin{array}{l}\text { Free from selective out- } \\
\text { come reporting? }\end{array}$ & Unclear risk & $\begin{array}{l}\text { Comment: no protocol or trial registry entry available and therefore not possi- } \\
\text { ble to assess }\end{array}$ \\
\hline Other risks of bias? & Low risk & Judgement comment: no evidence of other risks of bias \\
\hline
\end{tabular}

Davis 2003

Methods

Study aim: to determine if telemedicine improves eye examination rates in individuals with diabetes

Study design: parallel-group RCT

Participants

Country: USA

Setting: rural, federally funded, primary care practice in South Carolina

Total number of participants: 59

Percentage male: NR

Diabetes type: NR

Average age (SD): NR 
Davis 2003 (Continued)

Inclusion criteria: > 18 years with physician diagnosis of diabetes of any duration and on any form of treatment

Exclusion criteria: NR

Interventions

Intervention ( $\mathbf{n}=\mathbf{3 0}$ ): telemedicine retinal screening programme. Ophthalmologist at a distant site evaluated retinal photographs and consulted with the participant using real-time videoconferencing

Comparator $(\mathbf{n}=\mathbf{2 9})$ : usual care (reminded to schedule appointments with their usual eye care provider)

Duration: NR

Outcomes

Primary outcome: retinal examination attendance

Secondary outcomes: NR

Baseline screening attendance (control group): NR

\begin{tabular}{|c|c|c|}
\hline Notes & \multicolumn{2}{|c|}{$\begin{array}{l}\text { Date conducted: NR } \\
\text { Trial registration number: NR } \\
\text { Sources of funding: NR } \\
\text { Declaration of interest: NR }\end{array}$} \\
\hline \multicolumn{3}{|l|}{ Risk of bias } \\
\hline Bias & Authors' judgement & Support for judgement \\
\hline $\begin{array}{l}\text { Adequate sequence gener- } \\
\text { ation? }\end{array}$ & Unclear risk & Not reported \\
\hline $\begin{array}{l}\text { Adequate allocation con- } \\
\text { cealement? }\end{array}$ & Unclear risk & Not reported \\
\hline $\begin{array}{l}\text { Similar baseline outcome } \\
\text { measurements? }\end{array}$ & Unclear risk & Not reported \\
\hline $\begin{array}{l}\text { Similar baseline character- } \\
\text { istics? }\end{array}$ & Unclear risk & Not reported \\
\hline $\begin{array}{l}\text { Incomplete outcome data } \\
\text { addressed? }\end{array}$ & Unclear risk & Not reported \\
\hline $\begin{array}{l}\text { Knowledge of allocated in- } \\
\text { tervention prevented? }\end{array}$ & Unclear risk & Not reported \\
\hline $\begin{array}{l}\text { Protected against contam- } \\
\text { ination? }\end{array}$ & Low risk & $\begin{array}{l}\text { Judgement comment: it is unlikely that the control group received the inter- } \\
\text { vention }\end{array}$ \\
\hline $\begin{array}{l}\text { Free from selective out- } \\
\text { come reporting? }\end{array}$ & Unclear risk & $\begin{array}{l}\text { Judgement comment: no protocol or trial registry entry available and there- } \\
\text { fore not possible to assess }\end{array}$ \\
\hline Other risks of bias? & Unclear risk & Judgement comment: not possible to assess \\
\hline
\end{tabular}


Study aim: to evaluate a remote comprehensive diabetes self-management education intervention to improve adherence to American Diabetes Association (ADA) guidelines

Study design: parallel-group RCT

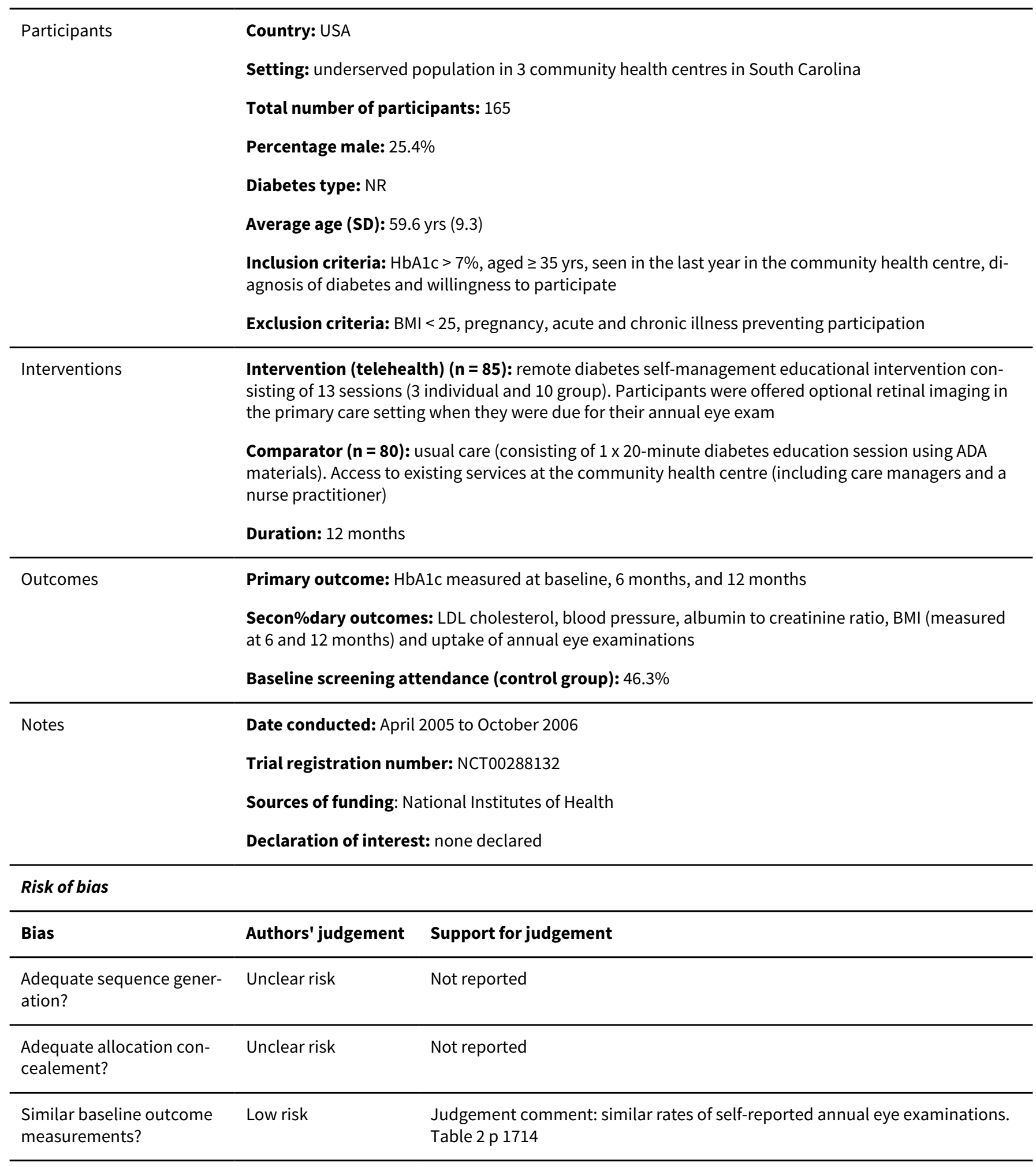


Dickinson 2014 (Continued)

en access to a website regarding quality improvements and received audit and feedback as in the other groups.

Duration: practice facilitation of 6 months (RAP) or 18 months (CQI)

Outcomes

Primary outcome: $\mathrm{HbAlc}$, blood pressure, lipids, process of care measured at baseline, 9 and 18 months (including diabetes-related visits to ophthalmologist)

Secondary outcomes: patient report (by survey) of their primary care experience

Baseline screening attendance (control group): 5.9\%

\begin{tabular}{ll}
\hline Notes & Date conducted: NR \\
& Trial registration number: NCT00414986 \\
& Sources of funding: National Institute of Diabetes and Kidney Diseases and the National Institute of \\
Mental Health & \\
Declaration of interest: none declared
\end{tabular}

\section{Risk of bias}

\begin{tabular}{|c|c|c|}
\hline Bias & Authors' judgement & Support for judgement \\
\hline $\begin{array}{l}\text { Adequate sequence gener- } \\
\text { ation? }\end{array}$ & Unclear risk & Not reported \\
\hline $\begin{array}{l}\text { Adequate allocation con- } \\
\text { cealement? }\end{array}$ & Low risk & $\begin{array}{l}\text { Judgement comment: unit of allocation by community health centre and allo- } \\
\text { cation performed prior to the start of the study }\end{array}$ \\
\hline $\begin{array}{l}\text { Similar baseline outcome } \\
\text { measurements? }\end{array}$ & Low risk & $\begin{array}{l}\text { Judgement comment: rates of dilated eye examinations were not statistically } \\
\text { different between study arms. Table } 2 \mathrm{p} 13\end{array}$ \\
\hline \multirow[t]{2}{*}{$\begin{array}{l}\text { Similar baseline character- } \\
\text { istics? }\end{array}$} & Unclear risk & $\begin{array}{l}\text { Quote: "...baseline HbA1c level, systolic blood pressure, and total cholesterol } \\
\text { level differed significantly across groups (all P<.05), with slightly better base- } \\
\text { line control of each in RAP practices." p } 11\end{array}$ \\
\hline & & $\begin{array}{l}\text { Judgement comment: unclear whether differences in baseline characteristics } \\
\text { would have influenced outcome }\end{array}$ \\
\hline $\begin{array}{l}\text { Incomplete outcome data } \\
\text { addressed? }\end{array}$ & Unclear risk & $\begin{array}{l}\text { Judgement comment: random sample of participants taken from each cluster } \\
\text { but missing data from some practices in chart audit }\end{array}$ \\
\hline $\begin{array}{l}\text { Knowledge of allocated in- } \\
\text { tervention prevented? }\end{array}$ & Unclear risk & Not reported \\
\hline $\begin{array}{l}\text { Protected against contam- } \\
\text { ination? }\end{array}$ & Low risk & $\begin{array}{l}\text { Judgement comment: allocation was by practice and it is unlikely that the } \\
\text { control group received the intervention }\end{array}$ \\
\hline $\begin{array}{l}\text { Free from selective out- } \\
\text { come reporting? }\end{array}$ & Low risk & $\begin{array}{l}\text { Judgement comment: reported outcomes consistent with trial registry } \\
\text { NCT00414986 }\end{array}$ \\
\hline Other risks of bias? & Unclear risk & Judgement comment: no evidence of other sources of bias \\
\hline
\end{tabular}


Dijkstra 2005

Methods

Study aim: to investigate whether a comprehensive strategy, involving both patients and professionals, with the introduction of a diabetes passport as a key component, improves diabetes care

Study design: cluster-RCT

\section{Participants}

Country: The Netherlands

Setting: 9 general hospitals throughout The Netherlands

Number of clusters: 9

Number of providers: 42

Total number of patients: 1350

Percentage male: $48 \%$

Diabetes type: types 1 and 2

Average age (SD): 58 yrs (15.5)

Inclusion criteria: all patients under the care of an internist for diabetic monitoring

Exclusion criteria: pregnancy; patients with low life expectancy

Intervention (4 clusters, $\mathbf{n}=\mathbf{6 0 0}$ patients): feedback on aggregated patient baseline data was given to the healthcare professionals. During an educational meeting with a national diabetes opinion leader, guidelines were issued on the prevention and treatment of diabetes complications as well as guidance on the use and dissemination of diabetes passports. The 'diabetes passport' is a patient-held booklet with important personal information that can be used to track results, record treatment targets and give information. The passport also records the medications used, results of laboratory and physical examinations and patient education. For patients additional educational meeting were organised

Comparator ( $\mathbf{5}$ clusters, $\mathbf{n}=\mathbf{7 5 0}$ patients): usual care (national diabetes guidelines issued to all hospitals during the intervention period)

Duration: 12 months

Outcomes

Primary outcome: measures consisted of process and outcome indicators taken from evidence-based Dutch guidelines on the treatment of diabetes and prevention of complications (including yearly examination of $\mathrm{HbA1c}$, creatinine, total cholesterol or total cholesterol/HDL ratio, urine for microalbuminuria, weight, BMI and blood pressure, as well as advice on smoking and physical exercise). The guidelines advise an eye examination every $1-2$ years (yearly in the case of those at higher risk of retinopathy)

\section{Secondary outcomes: NR}

Baseline screening attendance (control group): $84 \%$

Bias Authors' judgement Support for judgement


Dijkstra 2005 (Continued)

Adequate sequence gener- Unclear risk Not reported
ation?

Adequate allocation con- Low risk cealement?

Quote: "Random allocation was done by a person outside the research group and concealed from the investigators until the start of the intervention." p 128

\begin{tabular}{lll}
\hline $\begin{array}{l}\text { Similar baseline outcome } \\
\text { measurements? }\end{array}$ & Low risk & $\begin{array}{l}\text { Judgement comment: similar baseline eye examinations }<12 \text { months or }<24 \\
\text { months (see Table 2 p 131) }\end{array}$ \\
\hline $\begin{array}{l}\text { Similar baseline character- } \\
\text { istics? }\end{array}$ & Low risk & $\begin{array}{l}\text { Judgement comment: baseline characteristics similar across the 2 arms of the } \\
\text { study (see Tables } 1 \text { and 2 } \mathrm{p} \mathrm{131)}\end{array}$ \\
\hline $\begin{array}{l}\text { Incomplete outcome data } \\
\text { addressed? }\end{array}$ & High risk & $\begin{array}{l}\text { Judgement comment: high attrition (58.5\% and 55.7\% of those randomised to } \\
\text { intervention and control respectively were analysed) }\end{array}$ \\
\hline $\begin{array}{l}\text { Knowledge of allocated in- } \\
\text { tervention prevented? }\end{array}$ & Unclear risk & Not reported \\
\hline $\begin{array}{l}\text { Protected against contam- } \\
\text { ination? }\end{array}$ & Low risk & $\begin{array}{l}\text { Judgement comment: allocation was by hospital and it is unlikely that the } \\
\text { control group received the intervention }\end{array}$ \\
\hline $\begin{array}{l}\text { Free from selective out- } \\
\text { come reporting? }\end{array}$ & Unclear risk & $\begin{array}{l}\text { Judgement comment: no protocol or trial registry entry available and there- } \\
\text { fore not possible to assess }\end{array}$ \\
\hline \begin{tabular}{l} 
Other risks of bias? \\
\hline
\end{tabular} & Low risk & \begin{tabular}{l} 
Judgement comment: no evidence of other sources of bias \\
\hline
\end{tabular}
\end{tabular}

Dijkstra 2008

Methods Study aim: to investigate whether the introduction of a diabetes passport improves diabetes care

Study design: cluster-RCT

Participants

Country: The Netherlands

Setting: primary care practices in the middle and south regions of The Netherlands

Number of clusters: 40

Number of providers: 61

Total number of patients: 2059

Percentage male: $49.8 \%$

Diabetes type: types 2

Average age (SD): 63.4 yrs (9.6)

Inclusion criteria: all patients with type 2 diabetes $<80$ years under the care of a general practitioner

Exclusion criteria: those with a life expectancy < 1 year; patients who received their diabetes treatment in secondary care

\footnotetext{
Interventions

Intervention ( 20 clusters, $\mathbf{n}=\mathbf{1 0 0 4}$ participants): dissemination of diabetes passports. The 'diabetes passport'; is a patient-held booklet with important personal information that can be used to track results, record treatment targets and give information. The passport also records the medications used, results of laboratory and physical examinations and patient education. Additional patient education meetings were organised
} 
Dijkstra 2008 (Continued)

Comparator (20 clusters, $\mathbf{n}=\mathbf{1 0 5 5}$ participants): usual care (not specified)

Duration: 15 months

Primary outcome: self-reported use of the passport by participants
Secondary outcomes: process and outcome diabetes care indicators (including eye examination with-
in the previous 24 months)

Baseline screening attendance (control group): $72.2 \%$

\begin{tabular}{ll}
\hline Notes & Date conducted: NR \\
& Sources of funding: Netherlands Organisation for Health Research and Development \\
& Declaration of interest: NR
\end{tabular}

\section{Risk of bias}

\begin{tabular}{|c|c|c|}
\hline Bias & Authors' judgement & Support for judgement \\
\hline $\begin{array}{l}\text { Adequate sequence gener- } \\
\text { ation? }\end{array}$ & Unclear risk & Not reported \\
\hline $\begin{array}{l}\text { Adequate allocation con- } \\
\text { cealement? }\end{array}$ & Low risk & $\begin{array}{l}\text { Judgement comment: unit of allocation by community health centre and allo- } \\
\text { cation performed prior to the start of the study }\end{array}$ \\
\hline $\begin{array}{l}\text { Similar baseline outcome } \\
\text { measurements? }\end{array}$ & Low risk & $\begin{array}{l}\text { Judgement comment: similar baseline \% of eye examinations within } 24 \\
\text { months (see Table } 3 \text { p 75) }\end{array}$ \\
\hline \multirow[t]{2}{*}{$\begin{array}{l}\text { Similar baseline character- } \\
\text { istics? }\end{array}$} & Unclear risk & $\begin{array}{l}\text { Quote: "Comparison of the baseline data from the intervention and control } \\
\text { groups showed that there were some differences. The patients in the interven- } \\
\text { tion group were more often women and fewer monitored glucose themselves } \\
\text { than in the control group (Table 1)." }\end{array}$ \\
\hline & & $\begin{array}{l}\text { Judgement comment: baseline characteristic differences could have influ- } \\
\text { enced outcome }\end{array}$ \\
\hline $\begin{array}{l}\text { Incomplete outcome data } \\
\text { addressed? }\end{array}$ & Low risk & Judgement comment: eye screening data available for all participants \\
\hline $\begin{array}{l}\text { Knowledge of allocated in- } \\
\text { tervention prevented? }\end{array}$ & Unclear risk & Not reported \\
\hline $\begin{array}{l}\text { Protected against contam- } \\
\text { ination? }\end{array}$ & Low risk & $\begin{array}{l}\text { Judgement comment: allocation was by hospital and it is unlikely that the } \\
\text { control group received the intervention }\end{array}$ \\
\hline $\begin{array}{l}\text { Free from selective out- } \\
\text { come reporting? }\end{array}$ & Unclear risk & $\begin{array}{l}\text { Judgement comment: no protocol or trial registry entry available and there- } \\
\text { fore not possible to assess }\end{array}$ \\
\hline \multirow[t]{2}{*}{ Other risks of bias? } & Unclear risk & $\begin{array}{l}\text { Quote: "Table } 2 \text { shows that, in addition to the research intervention activities, } \\
\text { several control and intervention practices had initiated organizational inter- } \\
\text { ventions and revision of professional roles during the intervention period." p } \\
75\end{array}$ \\
\hline & & Judgement comment: not clear how these changes impacted on the outcome \\
\hline
\end{tabular}




\begin{tabular}{ll}
\hline Methods & Study aim: to evaluate the effectiveness and efficiency of a computerised diabetes register and man- \\
agement system on the quality of diabetes care
\end{tabular}
agement system on the quality of diabetes care

Study design: cluster-RCT

\begin{tabular}{|c|c|c|}
\hline Participants & $\begin{array}{l}\text { Number of providers } \\
\text { Total number of pati } \\
\text { Percentage male: } 53 \\
\text { Diabetes type: type } 2 \\
\text { Average age (SD): } 66 \\
\text { Inclusion criteria: pe } \\
\text { ing diabetes care excl } \\
\text { (GPs) and hospital } \\
\text { Exclusion criteria: NF}\end{array}$ & $\begin{array}{l}58 \\
\text { nts: } 3608 \\
\text { ple (11.5) } \\
\text { sively from stupe } 2 \text { diabetes appearing on the registers, aged }>35 \text { years and receiv- }\end{array}$ \\
\hline Interventions & \multicolumn{2}{|c|}{$\begin{array}{l}\text { Intervention ( } \mathbf{3 0} \text { clusters, } \mathbf{n}=\mathbf{1 6 7 4} \text { participants): computerised diabetes register incorporating a full } \\
\text { structured recall and management system, including individualised patient management prompts to } \\
\text { primary care clinicians based on locally-adapted, evidence-based guidelines } \\
\text { Comparator ( } \mathbf{2 8} \text { clusters, } \mathbf{n}=\mathbf{1 9 3 4} \text { participants): usual care (not specified) } \\
\text { Duration: } 15 \text { months }\end{array}$} \\
\hline Outcomes & \multicolumn{2}{|c|}{$\begin{array}{l}\text { Primary outcomes: clinical process and outcome variables held on the diabetes registers; patient-re- } \\
\text { ported outcomes (SF36 health status profile, the Newcastle Diabetes Symptoms Questionnaire and the } \\
\text { Diabetes Clinic Satisfaction Questionnaire); service and patient costs } \\
\text { Secondary outcomes: NR } \\
\text { Baseline screening attendance (control group): } 49.5 \%\end{array}$} \\
\hline Notes & $\begin{array}{l}\text { Date conducted: } 1 \mathrm{Ap} \\
\text { Trial registration nur } \\
\text { Sources of funding: } \mathrm{D} \\
\text { Declaration of intere } \\
\text { software used in the } \mathrm{s} \\
\text { Study protocol has be }\end{array}$ & $\begin{array}{l}2002 \text { to } 30 \text { June } 2003 \\
\text { ber: ISRCTN32042030 } \\
\text { abetes UK, and Northern and Yorkshire Regional NHS R\&D Office. } \\
\text { t: } 1 \text { of the authors was a partner in a software company that maintained the } \\
\text { Idy. The remaining authors declared no competing interests } \\
\text { n published: www.ncbi.nlm.nih.gov/pubmed/11914161 }\end{array}$ \\
\hline \multicolumn{3}{|l|}{ Risk of bias } \\
\hline Bias & Authors' judgement & Support for judgement \\
\hline $\begin{array}{l}\text { Adequate sequence gener- } \\
\text { ation? }\end{array}$ & Low risk & $\begin{array}{l}\text { Quote: "Randomisation was performed using electronically-generated random } \\
\text { numbers by the study statistician and was stratified by PCT and practice size." } \\
\text { p } 3\end{array}$ \\
\hline
\end{tabular}


Eccles 2007 (Continued)

$\begin{array}{ll}\begin{array}{l}\text { Adequate allocation con- } \\ \text { cealement? }\end{array} & \text { Low risk } \\ \end{array}$

Similar baseline outcome Low risk Judgement comment: similar \% of recorded fundoscopy at baseline
measurements?
measurements?

\section{Similar baseline character- Low risk istics?}

Quote: "Table 1 shows the baseline characteristics of control and intervention practices and patients. None of the differences in these variables between the intervention and control group are statistically significant." p 5

Judgement comment: although there was a high attrition for patient-reported outcomes, the register-derived outcomes were available for all participants

Judgement comment: data on fundoscopy obtained directly from the registry

Incomplete outcome data Low risk
addressed?

address
Knowledge of allocated in- Low risk tervention prevented?

\begin{tabular}{lll}
\hline $\begin{array}{l}\text { Protected against contam- } \\
\text { ination? }\end{array}$ & Low risk & $\begin{array}{l}\text { Judgement comment: allocation was by practice and it is unlikely that the } \\
\text { control group received the intervention }\end{array}$ \\
\hline $\begin{array}{l}\text { Free from selective out- } \\
\text { come reporting? }\end{array}$ & Low risk & $\begin{array}{l}\text { Judgement comment: reported outcomes consistent with trial registry } \\
\text { ISRCTN32042030 }\end{array}$ \\
\hline Other risks of bias? & Low risk & Judgement comment: no evidence of other sources of bias \\
\hline
\end{tabular}

\title{
Ellish 2011
}

Study aim: to compare the effects of a tailored (individualised) and targeted (generic) print interven-
tion in promoting dilated fundus examinations in older African Americans
tion in promoting dilated fundus examinations in older African Americans

Study design: parallel-group RCT

\section{Participants}

\section{Country: USA}

Setting: primary care

Total number of participants: 72 (sub-population with diabetes)

Percentage male: $25 \%$

Diabetes type: NR

Average age (SD): 72.4 yrs (6.3)

Inclusion criteria: African Americans aged $\geq 65$ yrs who had not had a dilated fundus examination in the last 2 years

\section{Exclusion criteria: NR}

Interventions

\begin{abstract}
Intervention ( $\mathbf{n}=39$ ): 'Tailored intervention'. Each participant received a 4-page newsletter including a testimonial designed to model eye examination behaviour and a barrier table to convey specific ideas to overcome barriers. The newsletter was specifically tailored by the addition of specific messages based on his/her responses to selected questions from a baseline questionnaire which identified barriers to screening and preventative health behaviours
\end{abstract}

Comparator $(\mathbf{n}=\mathbf{3 3})$ : 'Targeted intervention'. Participants received a standard newsletter with the same sections as the intervention group but without the tailored messages

Duration: 6 months 
Ellish 2011 (Continued)

Outcomes
Primary outcome: eye doctor confirmed dilated retinal examination at 6 months following randomisation

Secondary outcomes: predictors of retinal examination attendance

Baseline screening attendance (control group): $0 \%$

Notes

Date conducted: June 2007 and September 2008

Trial registration number: NCT00649766

Sources of funding: National Institutes of Health

Declaration of interest: none reported

Data on the sub-population with diabetes obtained from the author

\begin{tabular}{|c|c|c|}
\hline \multicolumn{3}{|l|}{ Risk of bias } \\
\hline Bias & Authors' judgement & Support for judgement \\
\hline $\begin{array}{l}\text { Adequate sequence gener- } \\
\text { ation? }\end{array}$ & Unclear risk & Not reported \\
\hline $\begin{array}{l}\text { Adequate allocation con- } \\
\text { cealement? }\end{array}$ & Unclear risk & Not reported \\
\hline $\begin{array}{l}\text { Similar baseline outcome } \\
\text { measurements? }\end{array}$ & Unclear risk & Not reported \\
\hline $\begin{array}{l}\text { Similar baseline character- } \\
\text { istics? }\end{array}$ & Low risk & $\begin{array}{l}\text { Quote "As reported in Table 2, at baseline the intervention groups were com- } \\
\text { parable for demographic and other variables." p } 1594\end{array}$ \\
\hline $\begin{array}{l}\text { Incomplete outcome data } \\
\text { addressed? }\end{array}$ & Low risk & $\begin{array}{l}\text { Judgement comment: low attrition. All participants accounted for (Figure } 1 \mathrm{p} \\
\text { 1594) }\end{array}$ \\
\hline $\begin{array}{l}\text { Knowledge of allocated in- } \\
\text { tervention prevented? }\end{array}$ & Unclear risk & Not reported \\
\hline $\begin{array}{l}\text { Protected against contam- } \\
\text { ination? }\end{array}$ & Low risk & $\begin{array}{l}\text { Judgement comment: it is unlikely that the control group received the tailored } \\
\text { intervention }\end{array}$ \\
\hline $\begin{array}{l}\text { Free from selective out- } \\
\text { come reporting? }\end{array}$ & Unclear risk & $\begin{array}{l}\text { Judgement comment: trial retrospectively registered and so not possible to } \\
\text { assess }\end{array}$ \\
\hline Other risks of bias? & Low risk & Judgement comment: no evidence of other sources of bias \\
\hline
\end{tabular}

Franco 2007

$\begin{array}{ll}\text { Methods } & \text { Study aim: to study the impact of an outreach visit by a diabetes specialist on general practitioners } \\ \text { management of type } 2 \text { diabetes }\end{array}$

Study design: cluster-RCT

Participants

Country: Réunion (French overseas territory)

Setting: General practices on the island of Réunion 
Franco 2007 (Continued)

Total number of clusters: 82

Number of providers: 82

Number of patients: 1581

Percentage male: $25 \%$

Diabetes type: type 2

Average age (SD): 59.9 (NR)

Inclusion criteria: GPs were selected if they had been working for 2 years or more and were likely to be employed for the duration of the study

Exclusion criteria: see above

\begin{abstract}
Interventions
Intervention ( $\mathbf{4 2}$ clusters, $\mathbf{n}=\mathbf{7 9 2}$ participants): 2 outreach visits by visiting GP with diabetes expertise. First visit consisted of a presentation on guideline recommendations, provision of teaching materials and clinical tools for diabetes assessment, e.g. esthesiometer. Second visit reinforced guideline recommendations and provided feedback on a questionnaire relating to 3 consecutive participants with diabetes seen following the first visit
\end{abstract}

Comparator (40 clusters, $\mathbf{n}=\mathbf{7 8 9}$ participants): usual care (not specified)

Duration: 2 outreach visits and outcomes measured within 6 months of the last visit

Primary outcome: compliance with processes of care recommendations for the management of type
2 diabetes including HbAlc, foot and fundus examination, creatinine clearance and assessment for pro-
teinuria/microalbuminuria which were measured within 6 months following delivery of intervention

Secondary outcomes: none

Baseline screening attendance (control group): $35 \%$

\begin{tabular}{|c|c|c|}
\hline Notes & \multicolumn{2}{|c|}{$\begin{array}{l}\text { Date conducted: NR } \\
\text { Trial registration number: NR } \\
\text { Sources of funding: NR } \\
\text { Declaration of interest: NR }\end{array}$} \\
\hline \multicolumn{3}{|l|}{ Risk of bias } \\
\hline Bias & Authors' judgement & Support for judgement \\
\hline $\begin{array}{l}\text { Adequate sequence gener- } \\
\text { ation? }\end{array}$ & Unclear risk & Not reported \\
\hline $\begin{array}{l}\text { Adequate allocation con- } \\
\text { cealement? }\end{array}$ & Low risk & $\begin{array}{l}\text { Judgement comment: unit of allocation by GP practice and allocation per- } \\
\text { formed prior to the start of the study }\end{array}$ \\
\hline $\begin{array}{l}\text { Similar baseline outcome } \\
\text { measurements? }\end{array}$ & Low risk & $\begin{array}{l}\text { Judgement comment: similar rates of retinopathy screening attendance at } \\
\text { baseline (see Table } 2 \text { p 2) }\end{array}$ \\
\hline $\begin{array}{l}\text { Similar baseline character- } \\
\text { istics? }\end{array}$ & Low risk & $\begin{array}{l}\text { Quote: "Le nombre, l'âge, le sex-ratio et le statut vis-à-vis de l'emploi des pa- } \\
\text { tients étaient semblables dans les deux groupes (tableau I). [The number, age, } \\
\text { sex ratio and employment status of patients were similar in both groups (Table } \\
\text { I)]" p } 2\end{array}$ \\
\hline
\end{tabular}


Franco 2007 (Continued) Incomplete outcome data High risk Judgement comment: high attrition (approx 30\% in both arms) addressed?

Knowledge of allocated in- High risk tervention prevented?
Judgement comment: GPs in the intervention group provided the data on retinopathy screening

\section{Protected against contam- Low risk} ination?
Quote "Dans le groupe témoin,contacté seulement à la fin de l'étude...[In the control group, contacted only at the end of the study],.." p 2

Judgement comment: allocation by cluster and unlikely that the control group received the intervention

\begin{tabular}{lll}
\hline $\begin{array}{l}\text { Free from selective out- } \\
\text { come reporting? }\end{array}$ & Unclear risk & $\begin{array}{l}\text { Judgement comment: no protocol or trial registry entry available and there- } \\
\text { fore not possible to assess. }\end{array}$ \\
\hline Other risks of bias? & Low risk & Judgement comment: no evidence of other risks of bias \\
\hline
\end{tabular}

Frei 2014

Methods Study aim: to test whether the implementation of elements of the 'Chronic Care Model (CCM)' by a specially-trained practice nurse leads to an improved cardiovascular risk profile among type 2 diabetes patients

Study design: cluster-RCT

Participants

Country: Switzerland

Setting: Primary care practices

Total number of clusters: 30

Number of providers: 30

Number of patients: 326

Percentage male: $57 \%$

Diabetes type: type 2

Average age (SD): 67 yrs (10.6)

Inclusion criteria: adults ( $>18$ years) with type 2 diabetes

Exclusion criteria: unable to read and understand the patient information form due to dementia, illiteracy or language skills. Patients with oncological diseases and/or an estimated life expectancy of less than six months due to severe diseases

Interventions

Intervention (15 clusters, $\mathbf{n}=\mathbf{1 6 4}$ participants): implementation of team care using elements of the Chronic Care Model (CCM) by a specially-trained practice nurse and using a computerised monitoring tool and decision support

Comparator (15 clusters, $\mathbf{n}=\mathbf{1 6 2}$ participants): usual care (not specified)

Duration: 12 months

Outcomes Primary outcome: HbAlc level

Secondary outcomes: guideline adherence (recommended treatment goals) including receiving at least 1 eye examination a year. Quality of life 
Frei 2014 (Continued)

Baseline screening attendance (control group): $64 \%$

Notes

Date conducted: 2010 to 2013

Trial registration number: ISRCTN05947538

Sources of funding: Swiss Academy for Medical Sciences; A. Menari AG, Switzerland

Declaration of interest: none declared

Study propocol has been published: www.ncbi.nlm.nih.gov/pubmed/20550650

\section{Risk of bias}

\begin{tabular}{|c|c|c|}
\hline Bias & Authors' judgement & Support for judgement \\
\hline $\begin{array}{l}\text { Adequate sequence gener- } \\
\text { ation? }\end{array}$ & Low risk & $\begin{array}{l}\text { Quote: "The PCPs who agreed to participate in the study were alphabetically } \\
\text { ordered by their family names in a list with numbers from } 1 \text { to } 30 \text {. An indepen- } \\
\text { dent research assistant, who was not involved in the study and was blind to } \\
\text { the identity of the PCPs, randomly allocated by statistical computer software } \\
\text { SPSS (version } 18.0 \text { ) } 15 \text { letters A and } 15 \text { letters B to numbers } 1-30 \text { and to the } \\
\text { corresponding PCPs, respectively. The assignment of the letters A and B to ei- } \\
\text { ther the intervention or control group was randomly conducted by a second } \\
\text { research assistant who drew blinded a ticket with the letters A or B and a tick- } \\
\text { et with the group allocation intervention or control group from an envelope." } \\
\text { p } 1041\end{array}$ \\
\hline
\end{tabular}

Adequate allocation con- Low risk cealement?
Quote: "We informed all PCPs about the group allocation after the inclusion of patients and baseline assessments to minimize selection bias." p 1041

\section{Similar baseline outcome High risk measurements?}

Judgement comment: different rates of retinopathy screening attendance at baseline (control 64\%, intervention 73.5\%) (see supplementary Table 2)

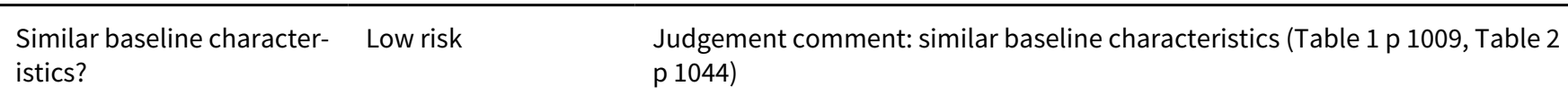

\author{
istics?
}

Incomplete outcome data Low risk

Judgement comment: data available for all providers and low rate of attrition in outcome data (see CONSORT diagram p 1042)
Knowledge of allocated in- Unclear risk tervention prevented?
Quote: "due to the study design, it was not possible to blind PCPs and practice nurses to group allocation, which might have influenced the results or might have led to a more pronounced effect of the intervention." p 1045

Judgement comment: unclear if would have affected diabetic retinopathy screening attendance

$\begin{array}{ll}\begin{array}{l}\text { Protected against contam- Low risk } \\ \text { ination? }\end{array} & \begin{array}{l}\text { Judgement comment: allocation was by practice and it is unlikely that the } \\ \text { control group received the intervention }\end{array}\end{array}$
ination?
Judgement comment: reported outcomes consistent with study protocol and trial registry ISRCTN05947538
Free from selective out- Low risk
come reporting?

Other risks of bias? Low risk
Judgement comment: no evidence of other risks of bias 
Frijling 2002 (Continued)

Study design: cluster-RCT

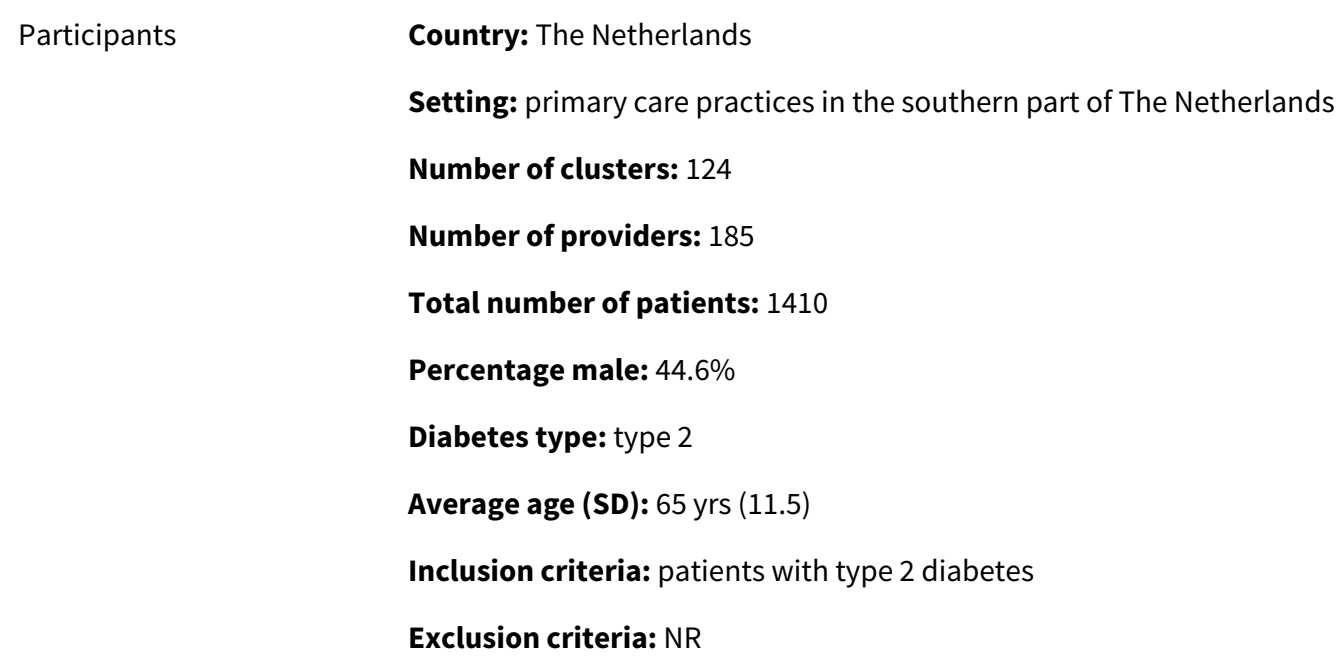
clinical decision-making about the diabetes guidelines issued by the Dutch College of General Practitioners and received outreach visits from facilitators. As part of the visits, the facilitator specifically addressed the clinical decision-making for patients with type 2 diabetes. The facilitator provided guidance, support, and educational materials to facilitate improvement

Comparator (62 clusters, $\mathbf{n}=\mathbf{7 0 7}$ participants): usual care (not specified)

Duration: 21 months

Outcomes Primary outcome: compliance rates for evidence-based indicators for management of patients with
type 2 diabetes (including eye examination in the past 24 months)

Secondary outcomes: NR

Baseline screening attendance (control group): $67 \%$

$\begin{array}{ll}\text { Notes } & \text { Date conducted: } 1996 \text { to } 1999 \\ \text { Trial registration number: NR } \\ \text { Sources of funding: Netherlands Heart Foundation. } \\ \text { Declaration of interest: NR }\end{array}$

\section{Risk of bias}

\begin{tabular}{lll}
\hline Bias & Authors' judgement & Support for judgement \\
\hline $\begin{array}{l}\text { Adequate sequence gener- } \\
\text { ation? }\end{array}$ & Low risk & $\begin{array}{l}\text { Quote: "A random-number generator was used to select permuted blocks with } \\
\text { a block size of four" p } 837\end{array}$ \\
\hline $\begin{array}{l}\text { Adequate allocation con- } \\
\text { cealement? }\end{array}$ & Low risk & $\begin{array}{l}\text { Quote: "The practices were numbered and the person responsible for the ran- } \\
\text { domization process was blind to the practice identities." p } 837\end{array}$ \\
\hline $\begin{array}{l}\text { Similar baseline outcome } \\
\text { measurements? }\end{array}$ & Low risk & Judgement comment: similar \% of eye examinations at baseline \\
\hline $\begin{array}{l}\text { Similar baseline character- } \\
\text { istics? }\end{array}$ & Low risk & $\begin{array}{l}\text { Quote: "The ages of the patients, the proportions of males and the proportions } \\
\text { of patients with uncontrolled blood glucose were found to be equally distrib- }\end{array}$
\end{tabular}


uted across the intervention and control groups at baseline and post-intervention measurement (Table 1)" p 838

Judgement comment: similar baseline clinical characteristics (see Table $2 \mathrm{p}$ 840)

\begin{tabular}{|c|c|c|}
\hline $\begin{array}{l}\text { Incomplete outcome data } \\
\text { addressed? }\end{array}$ & Low risk & $\begin{array}{l}\text { Judgement comment: low cluster attrition. High compliance with completion } \\
\text { of encounter forms }\end{array}$ \\
\hline
\end{tabular}

\begin{tabular}{|c|c|c|}
\hline $\begin{array}{l}\text { Knowledge of allocated in- } \\
\text { tervention prevented? }\end{array}$ & Low risk & $\begin{array}{l}\text { Judgement comment: although GPs completing the encounter forms follow- } \\
\text { ing each consultation were unmasked, the data were entered into a computer } \\
\text { by personnel blind to group allocation. }\end{array}$ \\
\hline
\end{tabular}

\begin{tabular}{|c|c|c|}
\hline $\begin{array}{l}\text { Protected against contam- } \\
\text { ination? }\end{array}$ & Low risk & $\begin{array}{l}\text { Judgement comment: allocation was by practice and it is unlikely that the } \\
\text { control group received the intervention }\end{array}$ \\
\hline
\end{tabular}
ination? control group received the intervention

\begin{tabular}{lll}
\hline $\begin{array}{l}\text { Free from selective out- } \\
\text { come reporting? }\end{array}$ & Unclear risk & $\begin{array}{l}\text { Judgement comment: no protocol or trial registry entry available and there- } \\
\text { fore not possible to assess }\end{array}$ \\
\hline Other risks of bias? & Low risk & Judgement comment: no evidence of other sources of bias \\
\hline
\end{tabular}

Gabbay 2006

$\begin{array}{ll}\text { Methods } & \text { Study aim: to measure the impact of a patient-oriented structured approach to care co-ordination } \\ \text { and patient education and counselling on improvements in BP, glycaemic control, lipids, complication } \\ \text { screening and diabetes-related distress }\end{array}$
screening and diabetes-related distress

Study design: parallel-group RCT

Participants

Country: USA

Setting: 2 primary care clinics of Penn State Hershey Medical Centre

Total number of participants: 332

Percentage male: $54.5 \%$

Diabetes type: type 2

Average age (SD): $64.5 \mathrm{yrs}(16.4)$

Inclusion criteria: patients with diabetes, $\geq 18$ years, identified by ICD 9 codes; 2 or more visits for diabetes within the last year

Exclusion criteria: patients unable to speak English; residents of nursing homes

Intervention ( $\mathbf{n}=\mathbf{1 5 0}$ ): nurse case manager implementing diabetes management using algorithms under the supervision of the participant's primary care physician (PCP) (a family physician or an internist). Goals were based on the ADA recommendations. The nurse case manager used behavioural goal-setting, established individualised care plan, provided participant self-management education and surveillance of participants, including phone calls to participants, referred patrticipants to a certified diabetes nurse educator or a dietitian where appropriate, ordered protocol-driven laboratory tests, tracked the outcomes using the computerised data registry and made therapeutic recommendations based on ADA diabetes guidelines with approval of the PCP

Comparator $(\mathbf{n}=\mathbf{1 8 2})$ : usual care by their PCP, and had no interaction with the nurse case manager

Duration: 12 months 
Gabbay 2006 (Continued)

Outcomes
Primary outcome: changes in BP, $\mathrm{HbAlc}$, lipids and complication screening process measures (including annual retinal screening)

Secondary outcomes: diabetes-related distress, as measured by the PAID questionnaire at 6 and 12 months. The PAID scale is a 20 -item measure of emotional adjustment to life with diabetes, with lower scores indicating better adjustment and coping with diabetes

Baseline screening attendance (control group): NR

\section{Risk of bias}

\begin{tabular}{|c|c|c|}
\hline Bias & Authors' judgement & Support for judgement \\
\hline \multirow[t]{2}{*}{$\begin{array}{l}\text { Adequate sequence gener- } \\
\text { ation? }\end{array}$} & High risk & $\begin{array}{l}\text { Quote: "A total of } 332 \text { patients were randomized (by method of odd and even } \\
\text { numbers) to either NCM intervention (intervention group), or a usual routine } \\
\text { care (control group)." p } 30\end{array}$ \\
\hline & & Judgement comment: inappropriate method of sequence generation \\
\hline $\begin{array}{l}\text { Adequate allocation con- } \\
\text { cealement? }\end{array}$ & Unclear risk & Not reported \\
\hline $\begin{array}{l}\text { Similar baseline outcome } \\
\text { measurements? }\end{array}$ & Unclear risk & Not reported \\
\hline \multirow[t]{2}{*}{$\begin{array}{l}\text { Similar baseline character- } \\
\text { istics? }\end{array}$} & Low risk & $\begin{array}{l}\text { Quote: "The intervention group }(\mathrm{n}=150) \text { and the control/ usual care group ( } \mathrm{n} \\
=182 \text { ) were statistically equivalent on baseline demographic and clinical char- } \\
\text { acteristics." }\end{array}$ \\
\hline & & p 31 \\
\hline $\begin{array}{l}\text { Incomplete outcome data } \\
\text { addressed? }\end{array}$ & Unclear risk & Judgement comment: attrition not reported \\
\hline $\begin{array}{l}\text { Knowledge of allocated in- } \\
\text { tervention prevented? }\end{array}$ & Unclear risk & Not reported \\
\hline $\begin{array}{l}\text { Protected against contam- } \\
\text { ination? }\end{array}$ & Low risk & $\begin{array}{l}\text { Judgement comment: it is unlikely that the control group received the inter- } \\
\text { vention }\end{array}$ \\
\hline $\begin{array}{l}\text { Free from selective out- } \\
\text { come reporting? }\end{array}$ & Unclear risk & $\begin{array}{l}\text { Judgement comment: no protocol or trial registry entry available and there- } \\
\text { fore not possible to assess }\end{array}$ \\
\hline Other risks of bias? & Low risk & $\begin{array}{l}\text { Judgement: although baseline characteristics were balanced across study } \\
\text { arms, only } 60 \% \text { of patients randomised to the intervention group agreed to } \\
\text { participate }\end{array}$ \\
\hline
\end{tabular}


Gabbay 2013

Study aim: to determine whether the addition of nurse case managers trained in motivational inter-
viewing would result in improved outcomes in type 2 diabetes patients at high risk of cardiovascular
complications

Study design: parallel-group RCT

Country: USA
Setting: 12 primary care clinics within 2 health systems in Central Pennsylvania
Total number of participants: 545
Percentage male: $37.8 \%$
Diabetes type: type 2
Average age (SD): 58 yrs (11)
Inclusion criteria: patients $18-75$ years with type 2 diabetes were eligible if they had 1 or more of the
following: (i) $\mathrm{HbAlc}>8.5 \%$; (ii) blood pressure $>140 / 90 \mathrm{mmHg}$; and/or (iii) Low-density lipoprotein
(LDL) $>130 \mathrm{mg} / \mathrm{dL}$

Exclusion criteria: could not communicate in either English or Spanish, or if residents of nursing homes

\begin{abstract}
Interventions
Intervention ( $\mathbf{n}=\mathbf{2 3 2}$ ): bilingual nurse case manager (NCM) met individually with participants at baseline, 2 and 6 weeks, at 3, 6 and 12 months and at least 6-monthly thereafter to review clinical laboratory test results, medication adherence and health-related lifestyle behaviour relating to managing their diabetes. The NCM also checked whether the participant was due for complications screening and reminded them of specialist visits
\end{abstract}

Comparator $(\mathbf{n}=\mathbf{3 1 3})$ : usual care (not specified)

Duration: 24 months

Outcomes $\quad$ Primary outcome: $\%$ of participants reaching the following outcomes 2 years after enrolment: (1) $\mathrm{HbA1C}<7$; (2) BP goal < 130/80; (3) LDL at goal < 100

Secondary outcomes: $\%$ of participants with yearly ophthalmologic exam,$\%$ of participants with yearly foot exam, \% of participants with assessment for nephropathy

Baseline screening attendance (control group): NR

\begin{tabular}{|c|c|c|}
\hline \multirow[t]{5}{*}{ Notes } & \multicolumn{2}{|c|}{ Date conducted: August 2006 to March 2008} \\
\hline & \multicolumn{2}{|c|}{ Trial registration number: $\mathrm{NCT00308386}$} \\
\hline & \multicolumn{2}{|c|}{ Sources of funding: National Institute of Diabetes and Kidney Diseases } \\
\hline & \multicolumn{2}{|c|}{ Declaration of interest: none declared } \\
\hline & \multicolumn{2}{|c|}{ Study protocol has been published: www.ncbi.nlm.nih.gov/pubmed/19328244 } \\
\hline \multicolumn{3}{|l|}{ Risk of bias } \\
\hline Bias & Authors' judgement & Support for judgement \\
\hline $\begin{array}{l}\text { Adequate sequence gener- } \\
\text { ation? }\end{array}$ & Unclear risk & Not reported \\
\hline $\begin{array}{l}\text { Adequate allocation con- } \\
\text { cealement? }\end{array}$ & Unclear risk & Not reported \\
\hline
\end{tabular}


Gabbay 2013 (Continued)

\begin{tabular}{lll}
$\begin{array}{l}\text { Similar baseline outcome } \\
\text { measurements? }\end{array}$ & Unclear risk & Not reported \\
\hline $\begin{array}{l}\text { Similar baseline character- } \\
\text { istics? }\end{array}$ & Low risk & $\begin{array}{l}\text { Quote: "Baseline characteristics of the study population are given in Table } \\
\text { 1. There were no significant differences in study measures between the two } \\
\text { groups." Table } 1 \mathrm{p} \mathrm{353}\end{array}$ \\
\hline $\begin{array}{l}\text { Incomplete outcome data } \\
\text { addressed? }\end{array}$ & High risk & $\begin{array}{l}\text { Judgement comment: high attrition and missing data unbalanced across } 2 \\
\text { arms of study (intervention 19\%, comparator 26\%) }\end{array}$ \\
\hline $\begin{array}{l}\text { Knowledge of allocated in- } \\
\text { tervention prevented? }\end{array}$ & Unclear risk & Not reported \\
\hline $\begin{array}{l}\text { Protected against contam- } \\
\text { ination? }\end{array}$ & Low risk & $\begin{array}{l}\text { Judgement comment: it is unlikely that the control group received the tele- } \\
\text { phone reminder }\end{array}$ \\
\hline $\begin{array}{l}\text { Free from selective out- } \\
\text { come reporting? }\end{array}$ & Low risk & $\begin{array}{l}\text { Judgement comment: reported outcomes consistent with trial registry } \\
\text { NCT00308386 }\end{array}$ \\
\hline $\begin{array}{l}\text { Other risks of bias? } \\
\text { High risk }\end{array}$ & $\begin{array}{l}\text { Judgement comment: per-protocol analysis. } N=42 \text { participants originally ran- } \\
\text { domised to the intervention arm were moved to the control group since they } \\
\text { did not receive the nurse Ml. Analysis and baseline data presented following } \\
\text { the switch }\end{array}$ \\
\hline
\end{tabular}

Glasgow 2005

Methods Study aim: to evaluate the effectiveness of a computer-assisted patient-centred intervention to improve the quality of diabetes care in primary care

Study design: cluster-RCT

\section{Participants}

\section{Country: USA}

Setting: family physicians and general internists insured by Sopic Insurance Co in Colorado

Number of clusters: 52

Number of providers: 52

Total number of patients: 886

Percentage male: $48 \%$

Diabetes type: type 2

Average age (SD): 62.9 yrs (12.7)

Inclusion criteria: adult patients $\geq 25$ years with type 2 diabetes and able to read English

Exclusion criteria: NR

Interventions

Intervention ( $\mathbf{2 4}$ clusters, $\mathbf{n}=\mathbf{4 6 9}$ participants): interactive computer programme recording when participant last received 11 items on the National Committee on Quality Assurance/American Diabetes Association Provider Recognition Program (PRP) measures, followed by a printout of a self-management action plan. This was overseen by a designated 'care manager' who met with the participant and reinforced self-management strategies by telephone

Comparator ( $\mathbf{2 8}$ clusters, $\mathbf{n}=\mathbf{4 1 7}$ participants): interactive computer programme recording when last received 11 items on the National Committee on Quality Assurance/American Diabetes Association 
Provider Recognition Program (PRP) measures, followed by a printout of a self-management action plan. Control participants did not meet or receive calls from the care manager

Duration: 12 months

Outcomes

Primary outcome: participant reports of provision of receiving the 11 items in the PRP measures (included dilated eye examination)

Secondary outcomes: Quality of life assessed using the revised 'Problem Areas in Diabetes Scale (PAID-2) and the Patient Health Questionnaire (PHQ); HbAlc and ratio of total cholesterol to HDL cholesterol levels

Baseline screening attendance (control group): $66.6 \%$

$\begin{array}{ll}\text { Notes } & \text { Date conducted: NR } \\ \text { Trial registration number: NR } \\ \text { Sources of funding: Agency for Health Research and Quality }\end{array}$

Declaration of interest: NR

\section{Risk of bias}

\begin{tabular}{|c|c|c|}
\hline Bias & Authors' judgement & Support for judgement \\
\hline $\begin{array}{l}\text { Adequate sequence gener- } \\
\text { ation? }\end{array}$ & Unclear risk & Not reported \\
\hline $\begin{array}{l}\text { Adequate allocation con- } \\
\text { cealement? }\end{array}$ & Low risk & $\begin{array}{l}\text { Judgement comment: unit of allocation by primary care practice and alloca- } \\
\text { tion performed prior to the start of the study }\end{array}$ \\
\hline $\begin{array}{l}\text { Similar baseline outcome } \\
\text { measurements? }\end{array}$ & Low risk & $\begin{array}{l}\text { Judgement comment: similar compliance with dilated eye examination atten- } \\
\text { dance at baseline (see Table } 2 \text { p 36) }\end{array}$ \\
\hline $\begin{array}{l}\text { Similar baseline character- } \\
\text { istics? }\end{array}$ & Low risk & $\begin{array}{l}\text { Quote "Initial analysis failed to show baseline differences between conditions } \\
\text { in any socioeconomic or baseline measures." p } 36\end{array}$ \\
\hline $\begin{array}{l}\text { Incomplete outcome data } \\
\text { addressed? }\end{array}$ & Unclear risk & $\begin{array}{l}\text { Judgement comment: high attrition ( } 19 \% \text { intervention, } 13 \% \text { control). Reasons } \\
\text { for missing data not given. Unclear if missing data would impact on outcome }\end{array}$ \\
\hline $\begin{array}{l}\text { Knowledge of allocated in- } \\
\text { tervention prevented? }\end{array}$ & Unclear risk & $\begin{array}{l}\text { Judgement comment: eye-screening outcome data based on self-reports and } \\
\text { not clear if outcome assessor was unmasked }\end{array}$ \\
\hline $\begin{array}{l}\text { Protected against contam- } \\
\text { ination? }\end{array}$ & Low risk & $\begin{array}{l}\text { Judgement comment: it is unlikely that the control group received the inter- } \\
\text { vention }\end{array}$ \\
\hline $\begin{array}{l}\text { Free from selective out- } \\
\text { come reporting? }\end{array}$ & Unclear risk & $\begin{array}{l}\text { Judgement comment: no protocol or trial registry entry available and there- } \\
\text { fore not possible to assess }\end{array}$ \\
\hline Other risks of bias? & Low risk & Judgement comment: no evidence of other sources of bias \\
\hline
\end{tabular}

Guldberg 2011

Methods Study aim: to evaluate the effect of an electronically-delivered feedback system on the quality of care for people with type 2 diabetes 
Study design: cluster-RCT

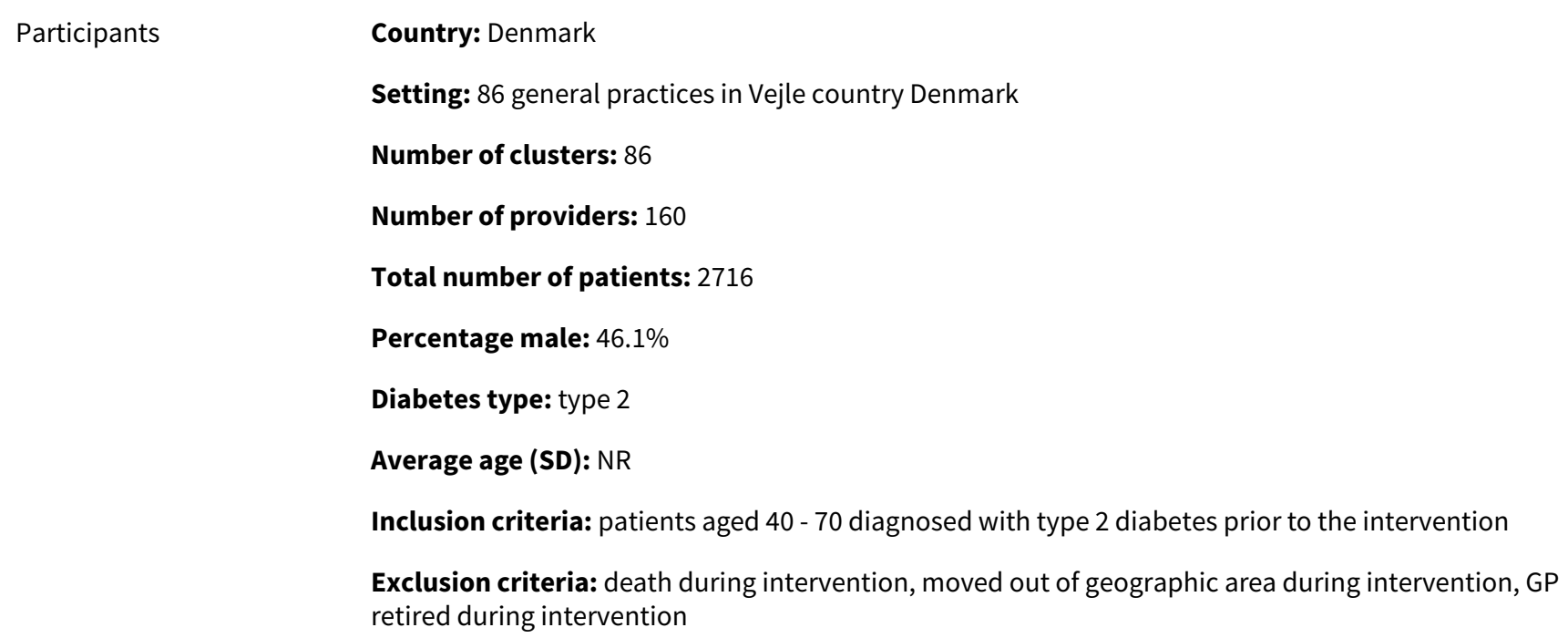

Secondary outcomes: qualitative study of how the intervention was used and received by the GPS

Baseline screening attendance (control group): NR

Trial registration number: NCT01009528

Sources of funding: Vejle County Quality Committee; Central Region Denmark Quality Committee; Danish Council for Independent Research; Tryg Foundation; Vissings Foundation; Danielsens Foundation; A. P.Moellers Foundation Promoting Medical Science

Declaration of interest: none declared

\section{Risk of bias}

\begin{tabular}{lll}
\hline Bias & Authors' judgement & Support for judgement \\
\hline $\begin{array}{l}\text { Adequate sequence gener- } \\
\text { ation? }\end{array}$ & Low risk & $\begin{array}{l}\text { Quote: "Randomization was unrestricted and was done using Stata software.." } \\
\text { p 326 }\end{array}$ \\
\hline $\begin{array}{l}\text { Adequate allocation con- } \\
\text { cealement? }\end{array}$ & Low risk & $\begin{array}{l}\text { Judgement comment: unit of allocation by GP practice and allocation per- } \\
\text { formed prior to the start of the study }\end{array}$ \\
\hline $\begin{array}{l}\text { Similar baseline outcome } \\
\text { measurements? }\end{array}$ & Unclear risk & Not reported \\
\hline
\end{tabular}


Guldberg 2011 (Continued)

Similar baseline character- Low risk Quote: "There were no statistically significantly differences concerning the istics?

quality of treatment between the people with Type 2 diabetes in the control and the intervention groups at baseline" Table 2 p 328

\begin{tabular}{|c|c|c|}
\hline $\begin{array}{l}\text { Incomplete outcome data } \\
\text { addressed? }\end{array}$ & Low risk & $\begin{array}{l}\text { Judgement comment: low attrition and missing data balanced across } 2 \text { arms } \\
\text { of study }\end{array}$ \\
\hline
\end{tabular}

Knowledge of allocated in- Low risk tervention prevented?

Quote:"In this study, most tasks were performed by one researcher. Therefore, and because a very visible tool like the electronic feedback system was tested, both blinding and allocation concealment were impossible in the study design." p 328

Judgement comment: data on annual eye examinations obtained from national registry and therefore unlikely to be influenced by knowledge of allocation

\begin{tabular}{ll}
\hline $\begin{array}{l}\text { Protected against contam- } \\
\text { ination? }\end{array}$ & Low risk \\
\end{tabular}

\begin{tabular}{lll}
\hline $\begin{array}{l}\text { Free from selective out- } \\
\text { come reporting? }\end{array}$ & Unclear risk & $\begin{array}{l}\text { Judgement comment: trial retrospectively registered and therefore not possi- } \\
\text { ble to assess }\end{array}$ \\
\hline Other risks of bias? & High risk & $\begin{array}{l}\text { Judgement comment: selection bias of providers as only 59\% of GPs accept- } \\
\text { ed invitation, and these may have been more willing to change according to } \\
\text { guidelines, or already had a high quality of care }\end{array}$ \\
\hline
\end{tabular}

\section{Gutierrez 2011}

Study aim: to assess the impact of shared medical appointments on the quality of care for Hispanic pa-
tients with type 2 diabetes attending a family medicine residency clinic

Study design: parallel-group RCT

\section{Participants}

\section{Country: USA}

Setting: single family medicine residency clinic

Total number of patients: 103

Percentage male: NR

Diabetes type: type 2

Average age (SD): NR

Inclusion criteria: Hispanic race/ethnicity, aged 18 years and older, diagnosis of type 2 diabetes with $\mathrm{HbA} 1 \mathrm{c} \geq 7 \%$

Exclusion criteria: dementia, current pregnancy or mothers who were breast-feeding

Interventions

Intervention ( $\mathbf{n}=\mathbf{5 0}$ ): shared medical appointments with a mean of 9 participants per group. Clinical team consisted of a resident or fellow researcher, faculty member, pharmacist, lead nurse, medical assistant, registration clerk, and social worker

Comparator ( $\mathbf{n}=\mathbf{5 3})$ : usual care (not specified)

Duration: 17 months 
Secondary outcomes: quality of life (Diabetes Quality of Life Brief Clinical Inventory) and diabetes knowledge (Diabetes Knowledge Questionnaire)

Baseline screening attendance (control group): $67.9 \%$

Notes

Date conducted: September 2006 to August 2007

Trial registration number: NR

Sources of funding: Department of Family and Community Medicine, University of Texas; Community Action Research Experience project funded by grant D58HP08301 from the Department of Health and Human Services Health Resources and Services Administration; foundation grant from the Texas Academy of Family Physicians

Declaration of interest: none declared

\section{Risk of bias}

\begin{tabular}{lll}
\hline Bias & Authors' judgement & Support for judgement \\
\hline $\begin{array}{l}\text { Adequate sequence gener- } \\
\text { ation? }\end{array}$ & Low risk & $\begin{array}{l}\text { Quote: "We assigned participants to an SMA group or a control group using a } \\
\text { table of random numbers." }\end{array}$ \\
\hline $\begin{array}{l}\text { Adequate allocation con- } \\
\text { cealement? }\end{array}$ & Unclear risk & Not reported \\
\hline
\end{tabular}

Similar baseline outcome Unclear risk $\quad$ Not reported
measurements?

\begin{tabular}{lll}
\hline $\begin{array}{l}\text { Similar baseline character- } \\
\text { istics? }\end{array}$ & Low risk & $\begin{array}{l}\text { Quote: "The SMA and control patients did not differ significantly by demo- } \\
\text { graphic, clinical, or other characteristics" p 213 }\end{array}$ \\
\hline $\begin{array}{l}\text { Incomplete outcome data } \\
\text { addressed? }\end{array}$ & Unclear risk & Not reported \\
\hline
\end{tabular}

Knowledge of allocated in- Unclear risk Not reported
tervention prevented?

\section{Protected against contam- Unclear risk} ination?
Quote: "...the possibility of a "halo effect" exists, where providers participating in the SMAs could have gained new knowledge and insight that allowed them to better treat patients in the control group. For example, a patient in the control group could have been advised by the pharmacist to ask his or her physician about switching to a different medication because a patient with similar clinical status in the SMA group was recently switched to that medication." $p$ 214

Judgement comment: unclear if potential for contamination would have influenced retinopathy screening attendance

\begin{tabular}{ll}
\hline $\begin{array}{l}\text { Free from selective out- } \\
\text { come reporting? }\end{array}$ & Unclear risk \\
\end{tabular}

\begin{tabular}{ll}
\hline Other risks of bias? $\quad$ Low risk Judgement comment: no evidence of other sources of bias \\
\hline
\end{tabular}

\section{Halbert 1999}

\begin{tabular}{ll}
\hline Methods & $\begin{array}{l}\text { Study aim: to determine whether multiple mailed patient reminders can produce an increase in atten- } \\
\text { dance for diabetic retinal examinations over that seen with a single reminder }\end{array}$ \\
\hline
\end{tabular}


Halbert 1999 (Continued)

Study design: parallel-group RCT

Country: USA
Setting: large network-based health maintenance organisation in California
Total number of participants: 23,740
Percentage male: $46.6 \%$
Diabetes type: NR
Average age (SD): NR
Inclusion criteria: all diabetic members $\geq 18$ years with no claim for a dilated fundus examination wh
were enrolled in Health Net, a large network-based health maintenance organisation (HMO) in Califor-
nia, during the study period
Exclusion criteria: NR
Intervention ( $\mathbf{n}=\mathbf{1 1 , 9 9 2 ) : ~ a t ~ b a s e l i n e , ~ p a r t i c i p a t i n g ~ m e d i c a l ~ g r o u p s ~ i n ~ t h e ~ H M O ~ n e t w o r k ~ r e c e i v e d ~}$
a letter explaining the programme, the current American Diabetes Association (ADA) guidelines for
retinal examinations, a sample physician letter, and lists of their patients with diabetes and their di-
abetic retinopathy screening exam status. The intervention group received reminders at 3 months, 6
months or 9 months after baseline if they had not had a dilated retinal examination according to the
HMO claims database. Mailing of reminders was verified by postal receipt

Comparator $(\mathbf{n}=\mathbf{1 1}, \mathbf{7 4 8})$ : at baseline, the diabetic members and their medical groups received the material described above. In addition, diabetic members who did not have a record of a diabetic retinopathy exam received educational materials and a report of their current retinopathy screening status directly from the HMO 2 weeks later

Duration: 12 months

Outcomes

Primary outcome: claims from either an ophthalmologist or optometrist using procedural terminology codes

Secondary outcomes: NR

Baseline screening attendance (control group): $0 \%$

Notes Date conducted: August 1996 to July 1997

Trial registration number: NR

Sources of funding: NR

Declaration of interest: NR

\section{Risk of bias}

\begin{tabular}{lll}
\hline Bias & Authors' judgement & Support for judgement \\
\hline $\begin{array}{l}\text { Adequate sequence gener- } \\
\text { ation? }\end{array}$ & Unclear risk & Not reported \\
\hline $\begin{array}{l}\text { Adequate allocation con- } \\
\text { cealement? }\end{array}$ & Unclear risk & Not reported \\
\hline $\begin{array}{l}\text { Similar baseline outcome } \\
\text { measurements? }\end{array}$ & Unclear risk & Not reported \\
\hline
\end{tabular}


Halbert 1999 (Continued)

Similar baseline character- Low risk Quote: "Table 1 describes the demographics of the eligible diabetic members istics? by sex and by age-group. There were no differences in sex and age-group distribution between the single and multiple intervention groups ( $P$ values were 0.225 and 0.063 , respectively)" p 753

\begin{tabular}{ll}
\hline $\begin{array}{l}\text { Incomplete outcome data } \\
\text { addressed? }\end{array}$ & Unclear risk \\
& $\begin{array}{l}\text { Judgement comment: members who disenrolled from the HMO during the } \\
\text { study period were excluded from the analysis. These were balanced across } \\
\text { both arms of the study ( } 18 \% \text { single reminder, } 17 \% \text { multiple reminder group). } \\
\text { Unclear if missing data would impact on outcome }\end{array}$
\end{tabular}

$\begin{array}{ll}\begin{array}{l}\text { Knowledge of allocated in- Low risk } \\ \text { tervention prevented? }\end{array} & \begin{array}{l}\text { Judgement comment: outcome data obtained from procedural codes and } \\ \text { therefore unlikely to be influenced by blinding }\end{array}\end{array}$
tervention prevented? therefore unlikely to be influenced by blinding

Protected against contam- Low risk Comparator group unlikely to receive the intervention
ination?

$\begin{array}{ll}\begin{array}{l}\text { Free from selective out- } \\ \text { come reporting? }\end{array} & \text { Unclear risk } \\ \end{array}$

\begin{tabular}{ll}
\hline Other risks of bias? Low risk Judgement comment: no evidence of other sources of bias \\
\hline
\end{tabular}

\section{Harris 2005}

Methods Study aim: to evaluate the effects of a continuing medical education intervention using teleconferencing on glycaemic control ( $\mathrm{HbA1c}$ ) and family physician adherence to national diabetes guidelines

Study design: cluster-RCT

Participants

Country: Canada

Setting: family physician clinics from 8 geographic regions in Canada

Number of clusters: 90

Number of providers: 90

Total number of patients: 660

Percentage male: $56 \%$

Diabetes type: type 2

Average age (SD): NR

Inclusion criteria: type 2 diabetes of at least 2 years' duration; aged $\geq 18$ years; a physician visit within the past year and competent to consent

Exclusion criteria: participating in the REACT2 study; pregnancy in previous 2 years module related to the management of type 2 diabetes based on national guidelines. Participants received an educational manual with defined learning objectives for each module, guideline recommendations, detailed clinical cases, and pertinent research articles. Flow sheets listing the recommended screening tests and clinical targets, designed to serve as reminders in participants' medical records, were also provided

Comparator (47 clusters, $\mathbf{n}=\mathbf{3 1 3}$ ): usual care (unspecified)

Duration: 3 months 
Harris 2005 (Continued)

Outcomes
Primary outcome: glycaemic control as measured by glycated haemoglobin (Hb A1c)

Secondary outcomes: medication management and physician adherence to clinical practice guideline complication screening recommendations (including eye examinations)

Baseline screening attendance (control group): NR
Date conducted: NR

Trial registration number: NR

Sources of funding: GlaxoSmithKline

Declaration of interest: 2 authors had been consultants and received honoraria for CME-related speaking engagements and research support from GlaxoSmithKline

\title{
Risk of bias
}

\begin{tabular}{|c|c|c|}
\hline Bias & Authors' judgement & Support for judgement \\
\hline $\begin{array}{l}\text { Adequate sequence gener- } \\
\text { ation? }\end{array}$ & Unclear risk & Not reported \\
\hline $\begin{array}{l}\text { Adequate allocation con- } \\
\text { cealement? }\end{array}$ & Low risk & $\begin{array}{l}\text { Judgement comment: unit of allocation by primary care practice and alloca- } \\
\text { tion performed prior to the start of the study }\end{array}$ \\
\hline $\begin{array}{l}\text { Similar baseline outcome } \\
\text { measurements? }\end{array}$ & Unclear risk & Not reported \\
\hline $\begin{array}{l}\text { Similar baseline character- } \\
\text { istics? }\end{array}$ & Low risk & $\begin{array}{l}\text { Judgement comment: gender balance, similar mean age at diagnosis and dis- } \\
\text { ease duration at baseline }\end{array}$ \\
\hline \multirow[t]{2}{*}{$\begin{array}{l}\text { Incomplete outcome data } \\
\text { addressed? }\end{array}$} & High risk & $\begin{array}{l}\text { Quote: "Of the } 90 \text { physicians randomly assigned, } 29(32 \%) \text { withdrew or were } \\
\text { unable to identify patients for audit." p } 90\end{array}$ \\
\hline & & Quote: "Patient consent per physician ranged from 17\% to 100\%" p 90 \\
\hline $\begin{array}{l}\text { Knowledge of allocated in- } \\
\text { tervention prevented? }\end{array}$ & Low risk & Quote: "Medical record auditors were blind to physician randomization." p 89 \\
\hline $\begin{array}{l}\text { Protected against contam- } \\
\text { ination? }\end{array}$ & Low risk & $\begin{array}{l}\text { Judgement comment: allocation was by practice and it is unlikely that the } \\
\text { control group received the intervention }\end{array}$ \\
\hline $\begin{array}{l}\text { Free from selective out- } \\
\text { come reporting? }\end{array}$ & Unclear risk & $\begin{array}{l}\text { Judgement comment: no protocol or trial registry entry available and there- } \\
\text { fore not possible to assess }\end{array}$ \\
\hline Other risks of bias? & Low risk & Judgement comment: no evidence of other sources of bias \\
\hline
\end{tabular}

Hayashino 2016

Methods Study aim: to evaluate the effect of a multifaceted intervention using the 'Achievable Benchmark of Care (ABC)' method for improving the technical quality of diabetes care in primary care settings

Study design: cluster-RCT

\author{
Participants \\ Country: Japan \\ Setting: primary care physicians within District Medical Associations
}


Hayashino 2016 (Continued)

\section{Total number of clusters: 22}

Number of providers: 192

Number of patients: 2236

Percentage male: $63 \%$

Diabetes type: type 2

Average age (SD): 56.5 yrs (5.9)

Inclusion criteria: type 2 diagnosis of diabetes prior to registration, aged 40 - 64 years and care provided by a single medical doctor in charge of the patient's diabetes treatment

Exclusion criteria: history of haemodialysis, hospitalisation, bed confinement, resident in a nursing home, blindness, history of lower limb amputation, history of diagnosis with a malignant tumour within the last 5 years, pregnancy or potential pregnancy

Interventions

Intervention (11 clusters, $\mathbf{n}=\mathbf{9 7 1}$ participants): physicians assigned to the intervention group were able to use a disease management system of monitoring and provided feedback on the quality of diabetes care, which was evaluated in terms of adherence to the 8 clinical indicators. Other intervention components included lifestyle advisors that provide reminders for regular visits and advice on lifestyle modifications by telephone or face-to-face

Comparator (11, $\mathbf{n}=\mathbf{1 2 6 5}$ participants): usual medical care (not specified)

Duration: 12 months

Outcomes

Primary outcome: quality of diabetes care score calculated on the outcomes of 8 quality indicators (including fundoscopy at least every 12 months)

Secondary outcomes: the effect of intervention on participant outcomes comprising HbA1c, systolic and diastolic blood pressure, and BMI

Baseline screening attendance (control group): $12.2 \%$

Date conducted: NR
Trial registration number: umin.ac.jp/ctr UMIN000002186
Sources of funding: Japan Agency for Medical Research and Development; Ministry of Health Labour
and Welfare
Declaration of interest: none declared
Study propocol has been published: Izumi, K., Hayashino, Y., Yamazaki, K. et al. Diabetol Int (2010) $1: 83$.
doi:10.1007/s13340-010-0015-6

\section{Risk of bias}

\begin{tabular}{lll}
\hline Bias & Authors' judgement & Support for judgement \\
\hline $\begin{array}{ll}\text { Adequate sequence gener- } \\
\text { ation? }\end{array}$ & Low risk & $\begin{array}{l}\text { Quote: 'The statistician, blind to the identities of the clusters, randomly allo- } \\
\text { cated } 0 \text { (control) or } 1 \text { (intervention) codes generated by statistical software, to } \\
\end{array}$ \\
& & \\
& &
\end{tabular}

\begin{tabular}{ll}
\hline $\begin{array}{l}\text { Adequate allocation con- Low risk } \\
\text { cealement? }\end{array}$ & $\begin{array}{l}\text { Judgement comment: unit of allocation by cluster and allocation performed } \\
\text { prior to the start of the study }\end{array}$
\end{tabular}

Similar baseline outcome Low risk measurements? Judgement comment: similar rates of retinopathy screening attendance at
baseline (Table 3 p 7 ) 
Hayashino 2016 (Continued)

Similar baseline character- Low risk Quote: "There was no statistical difference in baseline characteristics other istics? than the type of diabetes therapy between the IG and the CG; patients in the IG were more likely to receive diabetes medication $(P=0.049)$." p 5

\begin{tabular}{|c|c|c|}
\hline $\begin{array}{l}\text { Incomplete outcome data } \\
\text { addressed? }\end{array}$ & Low risk & $\begin{array}{l}\text { Judgement comment: data available for } 100 \% \text { providers and low rate of attri- } \\
\text { tion in outcome data (see CONSORT diagram p 5) }\end{array}$ \\
\hline
\end{tabular}

\begin{tabular}{lll}
\hline $\begin{array}{l}\text { Knowledge of allocated in- } \\
\text { tervention prevented? }\end{array}$ & Unclear risk & Not reported \\
\hline $\begin{array}{l}\text { Protected against contam- } \\
\text { ination? }\end{array}$ & Low risk & $\begin{array}{l}\text { Judgement comment: allocation by cluster and it is unlikely that the control } \\
\text { group received the intervention }\end{array}$ \\
\hline $\begin{array}{l}\text { Free from selective out- } \\
\text { come reporting? }\end{array}$ & Low risk & $\begin{array}{l}\text { Judgement comment: reported outcomes consistent with protocol (see lzumi } \\
\text { 2010) }\end{array}$ \\
\hline $\begin{array}{ll}\text { Other risks of bias? } & \text { Low risk }\end{array}$ & Judgement comment: no evidence of other risks of bias \\
\hline
\end{tabular}

Hermans 2013

Study aim: to assess the effect of 'benchmarking' on quality of primary care for patients with type 2 di-
abetes
Study design: cluster-RCT

Participants

Country: Belgium, Greece, Luxembourg, Portugal, Spain and the UK

Setting: general practitioner or hospital-based outpatient clinics to represent country-specific diabetes management practices

\section{Number of clusters: 477}

Number of providers: 477

Total number of patients: 4027

Percentage male: $55 \%$

Diabetes type: type 2

Average age (SD): $65.6 \mathrm{yrs}(10.8)$

Inclusion criteria: outpatients previously diagnosed with type 2 diabetes and $\geq 18$ years of age

Exclusion criteria: patients with gestational diabetes, patients with type 1 diabetes, those who were hospitalised as a result of their diabetes, participants in other clinical trials, and members of the Belgian Diabetes Convention (a quality assurance programme with benchmarked feedback)

Interventions

Intervention (293 clusters, $\mathbf{n}=\mathbf{2 5 0 9}$ participants): usual care consisting of routine monitoring, treatment and counselling of patients with type 2 diabetes with feedback benchmarked against other centres in each country

Comparator (184 clusters, $\mathbf{n}=\mathbf{1 5 1 8}$ participants): usual care (as intervention but without feedback)

Duration: 12 months 
Secondary outcomes: $\%$ of participants achieving targets in comparison with baseline of preventive screening, such as retinopathy, neuropathy; dietary counselling, microalbuminuria; smoking habits; BMI and physical activity

Baseline screening attendance (control group): $53 \%$

Date conducted: 2010
Trial registration number: NCT00681850
Sources of funding: editorial assistance and assistance with manuscript preparation and
Co-ordination was funded by AstraZeneca Belgium
Declaration of interest: HV is a full-time employee of AstraZeneca, all other authors declared that they
had sat on advisory boards or received honoraria from pharmaceutical companies
Study protocol has been published: www.ncbi.nlm.nih.gov/pubmed/21939502

\section{Risk of bias}

\begin{tabular}{|c|c|c|}
\hline Bias & Authors' judgement & Support for judgement \\
\hline $\begin{array}{l}\text { Adequate sequence gener- } \\
\text { ation? }\end{array}$ & Unclear risk & Not reported \\
\hline $\begin{array}{l}\text { Adequate allocation con- } \\
\text { cealement? }\end{array}$ & Low risk & $\begin{array}{l}\text { Quote: "Investigators were randomized by a centralized randomization pro- } \\
\text { cedure (What Health, Brussels, Belgium) to either a benchmarking group or a } \\
\text { control group" } \\
\text { p } 3389\end{array}$ \\
\hline $\begin{array}{l}\text { Similar baseline outcome } \\
\text { measurements? }\end{array}$ & Low risk & $\begin{array}{l}\text { Judgement comment: similar baseline retinopathy screening attendance }(< \\
10 \% \text { difference in baseline rates of annual ophthalmic examinations between } \\
\text { arms. Table } 2 \text { p 3393) }\end{array}$ \\
\hline $\begin{array}{l}\text { Similar baseline character- } \\
\text { istics? }\end{array}$ & Low risk & $\begin{array}{l}\text { Quote: "Baseline demographic and disease characteristics were similar be- } \\
\text { tween groups" p } 3390\end{array}$ \\
\hline $\begin{array}{l}\text { Incomplete outcome data } \\
\text { addressed? }\end{array}$ & High risk & $\begin{array}{l}\text { Judgement comment: } 23 \% \text { of clusters enrolled did not contribute to the final } \\
\text { analysis }\end{array}$ \\
\hline $\begin{array}{l}\text { Knowledge of allocated in- } \\
\text { tervention prevented? }\end{array}$ & Low risk & $\begin{array}{l}\text { Quote: "The sequence was concealed until the intervention was assigned, and } \\
\text { investigators were blinded to group assignment. Because randomization was } \\
\text { at the investigator level, blinding of patients was not applicable." p } 3389\end{array}$ \\
\hline $\begin{array}{l}\text { Protected against contam- } \\
\text { ination? }\end{array}$ & Low risk & $\begin{array}{l}\text { Judgement comment: allocation was by centre and it is unlikely that the con- } \\
\text { trol group received the intervention }\end{array}$ \\
\hline $\begin{array}{l}\text { Free from selective out- } \\
\text { come reporting? }\end{array}$ & Low risk & $\begin{array}{l}\text { Judgement comment: reported outcomes consistent with trial registry } \\
\text { NCT00681850 }\end{array}$ \\
\hline Other risks of bias? & High risk & Judgement comment: all authors had links to pharmaceutical companies \\
\hline
\end{tabular}

Herrin 2006

Methods

Study aim: to assess the effectiveness of diabetes resource nurse case management and physician profiling in improving diabetes care 
Herrin 2006 (Continued)

Study design: cluster-RCT

Participants

Country: USA

Setting: Family Medicine and Internal Medicine practices within the HealthTexas Provider Network (HTPN) - physician component of the Baylor Health Care System- Dallas-Fort Worth, Texas. HTPN- fee for service setting

Number of clusters: 22

Number of providers: 92

Total number of patients: 2155

Percentage male: $49.8 \%$

Diabetes type: NR

Average age (SD): 72.9 yrs (NR)

Inclusion criteria: patients aged $\geq 65$ years on 1 January 2000 , with a physician visit related to diabetes in 2000 and Medicare insurance coverage

Exclusion criteria: Patients who did not fulfil National Diabetes Quality Improvement Alliance criteria for diagnosis of diabetes mellitus; patients whose charts were not available for abstraction

Interventions

Intervention (claims plus MR group) (7 clusters, $\mathbf{n}=\mathbf{8 4 9}$ participants) Medicare claims feedback plus feedback on clinical measures from medical record (MR) abstraction

Intervention (claims plus MR plus DRS group) (8 clusters, $\mathrm{n}=\mathbf{6 5 4}$ participants): both types of feedback plus diabetes resource nurse (DRN)

Comparator (claims-only group) (7 clusters, $\mathbf{n}=652$ participants): Medicare claims feedback only

Duration: 24 months

Outcomes Primary outcome: HbA1c level; LDL level; diastolic and systolic blood pressures as dichotomous outcomes based on the ADA and National Diabetes Quality Improvement Alliance guidelines

Secondary outcomes: $\mathrm{HbA1C}, \mathrm{LDL}$, and diastolic and systolic blood pressures as continuous measures; processes of care measures including annual HbA1c assessment, annual lipid assessment, annual blood pressure measurement, annual eye exam, annual foot exam, and annual renal assessment

Baseline screening attendance (control group): $10.8 \%$

Notes

Date conducted: 2001

Trial registration number: NR

Sources of funding: American Diabetes Association; Pfizer, Inc; and the Baylor Health Care System

Declaration of interest: NR

\section{Risk of bias}

\begin{tabular}{|c|c|c|}
\hline Bias & Authors' judgement & Support for judgement \\
\hline $\begin{array}{l}\text { Adequate sequence gener- } \\
\text { ation? }\end{array}$ & Unclear risk & $\begin{array}{l}\text { Quote: "practices were stratified ... to ensure even distribution across arms.... } \\
\text { Within each stratum practices were sampled and randomized triplets to en- } \\
\text { sure even distribution" p } 97\end{array}$ \\
\hline & & $\begin{array}{l}\text { Judgement comment: not clear if method for sequence generation was appro- } \\
\text { priate }\end{array}$ \\
\hline
\end{tabular}


Herrin 2006 (Continued)

$\begin{array}{ll}\begin{array}{l}\text { Adequate allocation con- } \\ \text { cealement? }\end{array} & \text { Jow risk } \\ \text { prior to the start of the study }\end{array}$

Similar baseline outcome Low risk
measurements?

Judgement comment: similar attendance for annual eye examination based on Medicare claims Table 3 p 99 Similar baseline character- Low risk
istics?
Quote: "There were no differences in baseline clinical measures or in the data missing across study arms. There were no missing values for process measures, as patients were assumed to have failed the criteria if no record was found in the medical record or Medicare data." p 99

\begin{tabular}{lll}
\hline $\begin{array}{l}\text { Incomplete outcome data } \\
\text { addressed? }\end{array}$ & Low risk & $\begin{array}{l}\text { Quote: "There were no missing values for process measures, as patients were } \\
\text { assumed to have failed the criteria if no record was found in the medical } \\
\text { record or Medicare data." } \mathrm{p} 98\end{array}$ \\
\hline $\begin{array}{l}\text { Knowledge of allocated in- } \\
\text { tervention prevented? }\end{array}$ & Low risk & $\begin{array}{l}\text { Quote: "Both medical record and Medicare claims data were, however, collect- } \\
\text { ed by individuals blinded to patients' study arm assignments." p 101 }\end{array}$ \\
\hline $\begin{array}{l}\text { Protected against contam- } \\
\text { ination? }\end{array}$ & Low risk & $\begin{array}{l}\text { Judgement comment: allocation was by cluster and it is unlikely that the con- } \\
\text { trol group received the intervention }\end{array}$ \\
\hline $\begin{array}{l}\text { Free from selective out- } \\
\text { come reporting? }\end{array}$ & Unclear risk & $\begin{array}{l}\text { Judgement comment: no protocol or trial registry entry available and there- } \\
\text { fore not possible to assess }\end{array}$ \\
\hline $\begin{array}{l}\text { Other risks of bias? } \\
\text { Low risk }\end{array}$ & $\begin{array}{l}\text { Judgement comment: part-funded by pharmaceutical company, but states } \\
\text { that the company had no involvement in study design, data collection, data } \\
\text { analysis, or interpretation of data or asked to approve the final version of the } \\
\text { manuscript }\end{array}$
\end{tabular}

Hurwitz 1993

Methods Study aim: to evaluate the effectiveness and acceptability of centrally-organised prompting for co-or-
dinating community care of non-insulin-dependent diabetic patients

Study design: parallel-group RCT

$\begin{array}{ll}\text { Participants } & \text { Country: UK } \\ & \text { Setting: } 2 \text { hospital outpatient clinics, } 38 \text { general practices, and } 11 \text { optometrists in the catchment area } \\ \text { of a District General Hospital in Islington, London, UK }\end{array}$

Total number of participants: 181

Percentage male: $58 \%$

Diabetes type: type 2

Average age (SD): 62.6 yrs (10)

Inclusion criteria: mobile non-insulin-dependent diabetic patients under the age of 80 who had attended the District General Hospital diabetic clinics in the previous 2 years

Exclusion criteria: women of childbearing age; patients with 1 or more of 3 established significant diabetic complications, i.e. nephropathy with creatinine concentration $>150 \mu \mathrm{mol} / \mathrm{l}$; ischaemia severe enough to have resulted in gangrene or amputation, and retinopathy worse than background in 1 eye

Interventions Intervention $(\mathbf{n}=\mathbf{8 9})$ : prompting system using a database which sends requests to participants to provide blood and urine samples for testing at 6-monthly intervals. Results were incorporated within per- 
Hurwitz 1993 (Continued)

sonalised medical records which were sent to participants with a request to take them to their general practitioner within 10 days. General practitioner clinical assessments paralleled those of the hospital clinic. Participants not already under the care of a hospital eye clinic also received an annual eye test prompt and a map identifying local optometrists who performed dilated fundoscopy. Copies of optometry feedback are sent to the participant's general practitioner, who is thereby kept informed of eye assessments

Comparator ( $\mathbf{n}=\mathbf{9 2}$ ): usual care (hospital diabetes clinic review)

Duration: 6 months

Outcomes Primary outcome: number of diabetic reviews; glycaemic control; recording of processes of care (including random plasma glucose, HbAlc, eye screening)

Secondary outcomes: views of participants, participating GPs and optometrists

Baseline screening attendance (control group): $23.9 \%$

Notes Date conducted: April 1988 to October 1990

Trial registration number: NR

Sources of funding: NR

Declaration of interest: NR

\section{Risk of bias}

\begin{tabular}{|c|c|c|}
\hline Bias & Authors' judgement & Support for judgement \\
\hline $\begin{array}{l}\text { Adequate sequence gener- } \\
\text { ation? }\end{array}$ & Low risk & $\begin{array}{l}\text { Quote: "were randomised (by using Cambridge tables of random numbers)." p } \\
624\end{array}$ \\
\hline $\begin{array}{l}\text { Adequate allocation con- } \\
\text { cealement? }\end{array}$ & Unclear risk & Not reported \\
\hline $\begin{array}{l}\text { Similar baseline outcome } \\
\text { measurements? }\end{array}$ & Unclear risk & Not reported \\
\hline \multirow[t]{2}{*}{$\begin{array}{l}\text { Similar baseline character- } \\
\text { istics? }\end{array}$} & Low risk & $\begin{array}{l}\text { Quote: "Comparisons of control and prompted patient groups at the start of } \\
\text { the study are shown in table II. The groups were well matched for demograph- } \\
\text { ic variables and also for most important diabetic attributes, although mean } \\
\text { systolic blood pressure was recorded as } 9 \mathrm{~mm} \mathrm{Hg} \text { greater in the control group } \\
\text { (95\% confidence interval } 2.1 \text { to } 16.0 \mathrm{~mm} \mathrm{Hg} \text {; }=0.011 \text { ) and } 14 \text { patients in the } \\
\text { prompted group were documented as having signs of leg ischaemia compared } \\
\text { with only four controls } \times 2=5.7, \mathrm{df}=1 ; \mathrm{p}=0.017 \text { )." } \mathrm{p} 624\end{array}$ \\
\hline & & $\begin{array}{l}\text { Judgement comment: differences in baseline characteristics unlikely to influ- } \\
\text { ence outcome }\end{array}$ \\
\hline $\begin{array}{l}\text { Incomplete outcome data } \\
\text { addressed? }\end{array}$ & Low risk & $\begin{array}{l}\text { Quote: "At the end of October } 1990,94 \% \text { (170/181) of the general practitioner } \\
\text { notes for the study patients were traced." p } 624\end{array}$ \\
\hline $\begin{array}{l}\text { Knowledge of allocated in- } \\
\text { tervention prevented? }\end{array}$ & Unclear risk & Not reported \\
\hline $\begin{array}{l}\text { Protected against contam- } \\
\text { ination? }\end{array}$ & Low risk & Judgement comment: control participants unlikely to receive the intervention \\
\hline
\end{tabular}


Hurwitz 1993 (Continued)

Free from selective out- Unclear risk Judgement comment: no protocol or trial registry entry available and therecome reporting? fore not possible to assess

Other risks of bias?

Low risk

Judgement comment: no evidence of other sources of bias

Ilag 2003

Methods Study aim: to evaluate the impact of a systematic patient evaluation and patient and provider feedback on the processes and outcomes of diabetes care

Study design: cluster RCT

Participants Country: USA

Setting: university primary care internal medicine practices affiliated with a managed care organisation

Number of clusters: 9

Number of providers: 44

Total number of patients: 284

Percentage male: $47 \%$

Diabetes type: type 1 and 2

Average age (SD): 59 yrs (13.1)

Inclusion criteria: members of the managed care organisation with diabetes aged $\geq 18$ years

Exclusion criteria: NR

Interventions

Intervention (5 clusters, $\mathbf{n}=\mathbf{1 7 3}$ participants): ADAP visits in years 1 and 2 . This consisted of a 1 -hour focused encounter with non-physician providers within the primary care centre assessing key diabetes and cardiovascular health parameters measured (including fundus photography) and discussed with the participant by a certified diabetes educator. A tailored report with guideline-driven recommendations for care was sent to the participant's primary care provider and incorporated into the electronic patient record)

Comparator (4 clusters, $\mathbf{n}=\mathbf{1 1 1}$ participants): usual care in year 1, ADAP programme visits delivered in year 2

Duration: 24 months

Outcomes

Primary outcome: diabetes processes of care measures including: frequency of dilated retinal examinations, urine microalbumin measurements, foot examination, measurement of blood pressure HbAlc and LDL cholesterol

Secondary outcomes: participant and provider views of the ADAP programme

Baseline screening attendance (control group): $60.6 \%$

Date conducted: October 1999 to September 2016

Trial registration number: NR

Sources of funding: National Institutes of Health

Declaration of interest: NR 
Ilag 2003 (Continued)

Risk of bias

\begin{tabular}{|c|c|c|}
\hline Bias & Authors' judgement & Support for judgement \\
\hline $\begin{array}{l}\text { Adequate sequence gener- } \\
\text { ation? }\end{array}$ & Unclear risk & Method for cluster randomisation not reported \\
\hline $\begin{array}{l}\text { Adequate allocation con- } \\
\text { cealement? }\end{array}$ & Low risk & $\begin{array}{l}\text { Judgement comment: unit of allocation by primary care practice and alloca- } \\
\text { tion performed prior to the start of the study }\end{array}$ \\
\hline $\begin{array}{l}\text { Similar baseline outcome } \\
\text { measurements? }\end{array}$ & Unclear risk & Not reported \\
\hline $\begin{array}{l}\text { Similar baseline character- } \\
\text { istics? }\end{array}$ & Low risk & $\begin{array}{l}\text { Judgement comment: baseline characteristics balanced across the two arms } \\
\text { of the study (see Table } 1 \text { p 2724) }\end{array}$ \\
\hline $\begin{array}{l}\text { Incomplete outcome data } \\
\text { addressed? }\end{array}$ & High risk & $\begin{array}{l}\text { Judgement comment: high attrition (results reported for } 47 \% \text { of intervention } \\
\text { participants and } 64 \% \text { of comparison participants) }\end{array}$ \\
\hline $\begin{array}{l}\text { Knowledge of allocated in- } \\
\text { tervention prevented? }\end{array}$ & Unclear risk & Not reported \\
\hline $\begin{array}{l}\text { Protected against contam- } \\
\text { ination? }\end{array}$ & Low risk & $\begin{array}{l}\text { Quote: "We believe it was necessary to randomize by site to avoid within site } \\
\text { contamination." }\end{array}$ \\
\hline $\begin{array}{l}\text { Free from selective out- } \\
\text { come reporting? }\end{array}$ & Unclear risk & $\begin{array}{l}\text { Judgement comment: no protocol or trial registry entry available and there- } \\
\text { fore not possible to assess }\end{array}$ \\
\hline Other risks of bias? & Low risk & Judgement comment: no evidence of other sources of bias \\
\hline
\end{tabular}

Jacobs 2012

Methods Study aim: to assess whether pharmacists working with physicians and other healthcare providers in an ambulatory care setting can improve quality of care for patients with type 2 diabetes

Study design: parallel-group RCT

Participants Country: USA

Setting: single ambulatory general internal medicine setting

Total number of patients: 396

Percentage male: NR

Diabetes type: type 2

Average age (SD): 62.9 yrs (11)

Inclusion criteria: > 18 years with a documented $\mathrm{HbAlc}$ value $>8 \%$ obtained more than 6 months before the data acquisition date

Exclusion criteria: received primary care outside of the Lahey Clinic Burlington campus, were diagnosed with type 1 diabetes, had an HbAlc $<8 \%$ within 6 months of randomisation, were enrolled in any other pharmacist-run or diabetes management study, were receiving diabetes management by an outside endocrinologist, or were unable to adhere to scheduled follow-up 
Jacobs 2012 (Continued)

Interventions

Intervention ( $\mathbf{n}=\mathbf{1 9 5}$ ): pharmacist-participant clinic visits included obtaining a comprehensive medication review; performing targeted physical assessment; ordering laboratory tests; reviewing, modifying, and monitoring participants' medication therapy and providing detailed counselling on all therapies; facilitating self-monitoring of blood glucose; and providing reinforcement of dietary guidelines and exercise

Comparator $(\mathbf{n}=\mathbf{2 0 1})$ : usual care (not specified)

Duration: 12 months

Outcomes

Primary outcome: achieving targets for HbAlc (< $7 \%)$, LDL cholesterol $(<100 \mathrm{mg} / \mathrm{dL})$ and blood pressure $(<130 / 80 \mathrm{mmHg})$

Secondary outcomes: compliance with microvascular screening parameters including retinopathy, neuropathy and nephropathy

Baseline screening attendance (control group): NR

Notes Date conducted: 2003

Trial registration number: NCT00541606

Sources of funding: unrestricted medical grant from Pfizer

Declaration of interest: none declared

\section{Risk of bias}

\begin{tabular}{lll}
\hline Bias & Authors' judgement & Support for judgement \\
\hline $\begin{array}{l}\text { Adequate sequence gener- } \\
\text { ation? }\end{array}$ & Low risk & $\begin{array}{l}\text { Quote: "Eligible patients were randomized to either an intervention or control } \\
\text { group using a computer randomized sequence of ones and zeros" p 615 }\end{array}$ \\
\hline $\begin{array}{l}\text { Adequate allocation con- } \\
\text { cealement? }\end{array}$ & Unclear risk & Not report \\
\hline $\begin{array}{l}\text { Similar baseline outcome } \\
\text { measurements? }\end{array}$ & Unclear risk & Not reported \\
\hline $\begin{array}{l}\text { Similar baseline character- } \\
\text { istics? }\end{array}$ & Low risk & $\begin{array}{l}\text { Quote: "Baseline characteristics were similar between the two groups and re- } \\
\text { flect an obese white population of patients with diabetes, with a large percent- } \\
\text { age having comorbid medical conditions and existing microvascular complica- } \\
\text { tions (Table 1)." p 617 } \\
\text { Judgement comment: differences in baseline characteristics unlikely to affect } \\
\text { outcome }\end{array}$
\end{tabular}

\begin{tabular}{lll}
\hline $\begin{array}{l}\text { Incomplete outcome data } \\
\text { addressed? }\end{array}$ & High risk & $\begin{array}{l}\text { Judgement comment: per-protocol analysis (participants discontinuing inter- } \\
\text { vention were not included in the analysis). High attrition, unbalanced across } \\
\text { study arms }\end{array}$ \\
\hline $\begin{array}{l}\text { Knowledge of allocated in- } \\
\text { tervention prevented? }\end{array}$ & Unclear risk & Not reported \\
\hline $\begin{array}{l}\text { Protected against contam- } \\
\text { ination? }\end{array}$ & Low risk & $\begin{array}{l}\text { Judgement comment: allocation was by cluster and it is unlikely that the con- } \\
\text { trol group received the intervention }\end{array}$ \\
\hline $\begin{array}{l}\text { Free from selective out- } \\
\text { come reporting? }\end{array}$ & Unclear risk & $\begin{array}{l}\text { Judgement comment: trial retrospectively registered and therefore not possi- } \\
\text { ble to assess }\end{array}$ \\
\hline
\end{tabular}


Jacobs 2012 (Continued)

Other risks of bias? High risk Judgement comment: risk of selection bias

Quote: "Patients who agreed to participate in the study were likely more motivated to adhere to a diabetes treatment program. Although the control patients had to have obtained a minimum number of laboratory tests to be included, some patients in this group may not have participated in the study and may have been a less motivated group than the intervention group." p 619 that integrates patient-centred lifestyle counselling into structured diabetes care

Study design: cluster-RCT

\section{Participants}

Country: The Netherlands

Setting: general practices in the south-eastern part of The Netherlands

Number of clusters: 58

Number of providers: 58

Total number of patients: 940

Percentage male: $54.9 \%$

Diabetes type: type 2

Average age (SD): NR

Inclusion criteria: patients aged $<85$ years with a $\mathrm{HbAlc}>7 \%$ and a $\mathrm{BMI}>25 \mathrm{~kg} / \mathrm{m}^{2}$

Exclusion criteria: complex comorbidity and treatment in hospital gramme consisting of (a) training in lifestyle counselling based on motivational interviewing; (b) tools for structuring diabetes care, such as training in agenda setting, a local diabetes protocol based on the national guidelines and a social map for lifestyle support; (c) instruction on record-keeping to integrate lifestyle counselling into general practice; and (d) introduction of tools to sustain improvements including an instruction chart (reminder), regular telephone follow-ups with the target participants, a help desk that also enquired proactively about the progress of diabetes management, and a follow-up meeting for the nurses

Comparator (29 clusters, $\mathbf{n}=\mathbf{5 1 8}$ participants): nurses in the comparator group were advised to administer care consistent with current diabetes guidelines

Duration: 14 months

Outcomes

Primary outcome: $\mathrm{HbAlc}$ and reported changes in lifestyle related to diet and physical activity

Secondary outcomes: other diabetes processes of care recommendations (including eye examination); quality of life (using EQ-5D)

Baseline screening attendance (control group): NR

Notes Date conducted: 2008

Trial registration number: ISRCTN68707773

Sources of funding: ZonMW-the Netherlands Organization for Health Research and Development 
Jansink 2013 (Continued)

Declaration of interest: none declared

\title{
Risk of bias
}

\begin{tabular}{|c|c|c|}
\hline Bias & Authors' judgement & Support for judgement \\
\hline $\begin{array}{l}\text { Adequate sequence gener- } \\
\text { ation? }\end{array}$ & Unclear risk & Not reported \\
\hline $\begin{array}{l}\text { Adequate allocation con- } \\
\text { cealement? }\end{array}$ & Low risk & $\begin{array}{l}\text { Judgement comment: unit of allocation by general practice and allocation } \\
\text { performed prior to the start of the study }\end{array}$ \\
\hline $\begin{array}{l}\text { Similar baseline outcome } \\
\text { measurements? }\end{array}$ & Unclear risk & Not reported \\
\hline $\begin{array}{l}\text { Similar baseline character- } \\
\text { istics? }\end{array}$ & Low risk & Judgement comment: similar baseline characteristics. Table 1 p123 \\
\hline \multirow[t]{2}{*}{$\begin{array}{l}\text { Incomplete outcome data } \\
\text { addressed? }\end{array}$} & High risk & $\begin{array}{l}\text { Quote: "A limitation of the study is the loss to follow-up in the lifestyle mea- } \\
\text { sures from the patient questionnaire" p } 125\end{array}$ \\
\hline & & $\begin{array}{l}\text { Judgement comment: large losses to follow-up, reasons not provided. Out- } \\
\text { comes reported on } 47.8 \% \text { of eligible participants }\end{array}$ \\
\hline $\begin{array}{l}\text { Knowledge of allocated in- } \\
\text { tervention prevented? }\end{array}$ & Unclear risk & Not reported \\
\hline $\begin{array}{l}\text { Protected against contam- } \\
\text { ination? }\end{array}$ & Low risk & $\begin{array}{l}\text { Judgement comment: allocation was by cluster and it is unlikely that the con- } \\
\text { trol group received the intervention }\end{array}$ \\
\hline $\begin{array}{l}\text { Free from selective out- } \\
\text { come reporting? }\end{array}$ & Low risk & $\begin{array}{l}\text { Judgement comment: reported outcomes consistent with trial registry } \\
\text { ISRCTN68707773 }\end{array}$ \\
\hline Other risks of bias? & Low risk & Judgement comment: no evidence of other sources of bias \\
\hline
\end{tabular}

Kirwin 2010

Methods Study aim: to assess whether pharmacists working with primary care physicians can improve the quality of diabetes care

Study design: cluster-RCT

Participants

\author{
Country: USA \\ Number of clusters: 8 \\ Number of providers: 72 \\ Total number of patients: 346 \\ Percentage male: $34.2 \%$ \\ Diabetes type: types 1 and 2 \\ Average age (SD): 63 yrs (NR)
}

Setting: single hospital-based primary care practice 
Kirwin 2010 (Continued)

Inclusion criteria: 18 years or older; diagnosis of diabetes; patient had a primary care physician practising within the study clinic; seen in the practice at least once during the 2 years prior to the start of the study

\section{Exclusion criteria: NR}

Intervention (4 clusters, $\mathbf{n}=\mathbf{1 7 1}$ participants): primary care physicians received a personalised let-
ter from a pharmacist for participants with upcoming clinic visits. The letter contained information ex-
tracted from the electronic patient record on overdue testing and drug therapy to achieve diabetes-re-
lated treatment targets

Comparator (4 clusters, $\mathbf{n}=\mathbf{1 7 5}$ participants): usual care (not specified)

Duration: recommendation letter sent and outcome determined 30 days after the visit to the primary care physician

Primary outcome: process measure of annual HbAlc testing
Secondary outcomes: 4 processes of care measures (including annual eye examination) and 3 bio-
marker measures $(\mathrm{HbAlc}<7 \%, \mathrm{LDL}<100 \mathrm{mg} / \mathrm{dL}, \mathrm{BP}<130 / 80$ )

Baseline screening attendance (control group): $37.1 \%$

\begin{tabular}{ll}
\hline Notes & Date conducted: 2004 \\
& Trial registration number: NCT00122421 \\
& Sources of funding: none \\
& Declaration of interest: none declared
\end{tabular}

Risk of bias

\begin{tabular}{|c|c|c|}
\hline Bias & Authors' judgement & Support for judgement \\
\hline \multirow[t]{2}{*}{$\begin{array}{l}\text { Adequate sequence gener- } \\
\text { ation? }\end{array}$} & Low risk & $\begin{array}{l}\text { Quote: "In July 2003, we identified } 1,349 \text { patients meeting these criteria and } \\
\text { used a random number generator to randomly select } 560 \text { being cared for by } 72 \\
\text { PCPs for inclusion in the study (Figure } 1 \text { )." p } 106\end{array}$ \\
\hline & & $\begin{array}{l}\text { Quote: "We randomized the intervention at the level of clinical suites within } \\
\text { the study practice immediately after patients were identified in July 2003." p } \\
106\end{array}$ \\
\hline $\begin{array}{l}\text { Adequate allocation con- } \\
\text { cealement? }\end{array}$ & Low risk & $\begin{array}{l}\text { Judgement comment: unit of allocation at the level of the cluster and alloca- } \\
\text { tion performed prior to the start of the study }\end{array}$ \\
\hline $\begin{array}{l}\text { Similar baseline outcome } \\
\text { measurements? }\end{array}$ & Low risk & $\begin{array}{l}\text { Judgement comment: similar baseline annual eye examination in intervention } \\
\text { and control ( } 38 \% \text { vs } 37.1 \%)\end{array}$ \\
\hline $\begin{array}{l}\text { Similar baseline character- } \\
\text { istics? }\end{array}$ & Low risk & $\begin{array}{l}\text { Judgement comment: similar baseline characteristics. Baseline imbalance in } \\
\text { annual lipid profile assessment but unlikely to influence outcome. }\end{array}$ \\
\hline $\begin{array}{l}\text { Incomplete outcome data } \\
\text { addressed? }\end{array}$ & High risk & $\begin{array}{l}\text { Judgement comment: per-protocol analysis, baseline based on those } \\
\text { analysed. Reasons for missing data not provided }\end{array}$ \\
\hline $\begin{array}{l}\text { Knowledge of allocated in- } \\
\text { tervention prevented? }\end{array}$ & Unclear risk & Not reported \\
\hline $\begin{array}{l}\text { Protected against contam- } \\
\text { ination? }\end{array}$ & Low risk & $\begin{array}{l}\text { Judgement comment: allocation by cluster and it is unlikely that the control } \\
\text { group received the intervention }\end{array}$ \\
\hline
\end{tabular}


Kirwin 2010 (Continued)

$\begin{array}{ll}\text { Free from selective out- } & \text { Low risk } \\ \text { come reporting? } & \text { NCT00122421 }\end{array}$
come reporting? NCT00122421

Other risks of bias?

$\begin{array}{ll}\text { Methods } & \text { Study aim: to evaluate the effects of a collaborative case management intervention for patients with } \\ \text { poorly-controlled type } 2 \text { diabetes on glycaemic control, intermediate cardiovascular outcomes, satis- } \\ \text { faction with care, and resource utilisation }\end{array}$

Study design: parallel-group RCT

Setting: Department of Veterans Affairs (VA) Medical Centres

Total number of participants: 246

Percentage male: $96.5 \%$

Diabetes type: type 2

Average age (SD): 61 yrs (10.5)

Inclusion criteria: patients with at least 1 prescription for an oral hypoglycemic agent, insulin, or blood glucose monitoring supplies filled in the previous 12 months; most recent (HbA1c) $\geq 8.5 \%$ (within the last year); general medicine clinic visit scheduled between May 1999 and January 2000

Exclusion criteria: < 18 years; type 1 diabetes or were diagnosed before the age of 30 years; had no telephone; did not speak English; were not competent for interview; reported primary source of diabetes care outside the VA; were being treated for cancer (other than non-melanoma skin cancer); had kidney failure, symptomatic heart failure, liver disease, or blindness; spent winter at another residence; or planned to move their primary care providers, monitoring and co-ordinating care through the use of telephone contacts, collaborative goal setting, and treatment algorithms

Comparator $(\mathbf{n}=\mathbf{1 2 3})$ : provision of educational materials and usual care by their primary care physician

Duration: 18 months

Outcomes Primary outcome: glycaemic control, as measured by HbA1c level; control of LDL cholesterol; and blood pressure

Secondary outcomes: health status and participant satisfaction were assessed using a self-administered written survey, which included the Short Form Health Survey for Veterans and the Patient Satisfaction Questionnaire-Form II (general satisfaction subscale); demographic characteristics, receipt of eye screening, aspirin use, and healthcare services received outside the VA

Baseline screening attendance (control group): $67.5 \%$ 
Krein 2004 (Continued)

Sources of funding: Office of Research and Development, Health Services Research and Development Service, Department of Veterans Affairs; Michigan Diabetes Research and Training Center Grant; National Institute of Diabetes and Digestive and Kidney Diseases, National Institutes of Health

Declaration of interest: NR

\section{Risk of bias}

\begin{tabular}{|c|c|c|}
\hline Bias & Authors' judgement & Support for judgement \\
\hline $\begin{array}{l}\text { Adequate sequence gener- } \\
\text { ation? }\end{array}$ & Low risk & $\begin{array}{l}\text { Quote: "One member of a matched pair, within one of four possible blocks/ } \\
\text { cells (site by baseline HbA1C level), was then assigned randomly to the case } \\
\text { management group and the other to the control group by the project manager } \\
\text { who had no knowledge about the patients other than site and baseline HbA1c } \\
\text { level." p } 733\end{array}$ \\
\hline $\begin{array}{l}\text { Adequate allocation con- } \\
\text { cealement? }\end{array}$ & Low risk & $\begin{array}{l}\text { Quote: "One member of a matched pair, within one of four possible blocks/ } \\
\text { cells (site by baseline HbA1C level), was then assigned randomly to the case } \\
\text { management group and the other to the control group by the project manager } \\
\text { who had no knowledge about the patients other than site and baseline HbA1c } \\
\text { level." p } 733\end{array}$ \\
\hline $\begin{array}{l}\text { Similar baseline outcome } \\
\text { measurements? }\end{array}$ & Low risk & $\begin{array}{l}\text { Judgment comment: similar baseline attendance for diabetic retinopathy } \\
\text { screening ( } 9 \% \text { baseline difference, see Table } 1 \text { p 735) }\end{array}$ \\
\hline $\begin{array}{l}\text { Similar baseline character- } \\
\text { istics? }\end{array}$ & Low risk & $\begin{array}{l}\text { Quote: "The baseline attributes of the intervention and control groups were } \\
\text { similar (Table 1). Except for having a higher percentage of non white partici- } \\
\text { pants, study enrollees were demographically representative of VA ambulatory } \\
\text { patients." p } 734\end{array}$ \\
\hline $\begin{array}{l}\text { Incomplete outcome data } \\
\text { addressed? }\end{array}$ & Low risk & $\begin{array}{l}\text { Judgement comment: low attrition, balanced across the arms of the study and } \\
\text { missing data accounted for }\end{array}$ \\
\hline $\begin{array}{l}\text { Knowledge of allocated in- } \\
\text { tervention prevented? }\end{array}$ & Low risk & $\begin{array}{l}\text { Judgement comment: eye-screening data obtained from VA medical informa- } \\
\text { tion system and therefore unlikely to be influenced by lack of masking }\end{array}$ \\
\hline $\begin{array}{l}\text { Protected against contam- } \\
\text { ination? }\end{array}$ & Low risk & Judgement comment: control group unlikely to have received the intervention \\
\hline $\begin{array}{l}\text { Free from selective out- } \\
\text { come reporting? }\end{array}$ & Unclear risk & $\begin{array}{l}\text { Judgement comment: no protocol or trial registry entry available and there- } \\
\text { fore not possible to assess }\end{array}$ \\
\hline Other risks of bias? & Low risk & Judgement comment: no evidence of other sources of bias \\
\hline
\end{tabular}

Lafata 2002

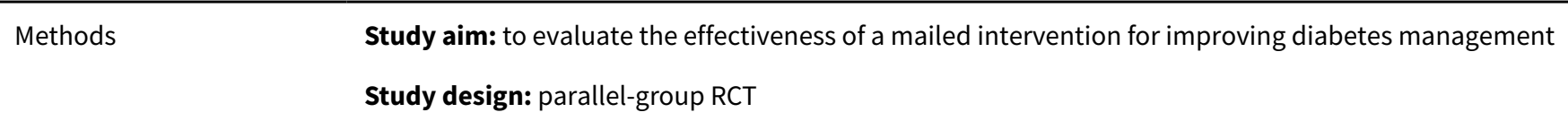

Participants

Country: USA

Setting: multi-specialty primary care group practice

Total number of participants: 3309

Percentage male: $47.8 \%$ 
Lafata 2002 (Continued)

Diabetes type: NR

Average age (SD): $59.8 \mathrm{yrs}$ (NR)

Inclusion criteria: patients aged $\geq 18$ yrs with diabetes, aligned to a primary care physician within a multispeciality practice

Exclusion criteria: none

Interventions

Intervention ( $\mathbf{n}=\mathbf{1 6 4 1}$ ): mailed reminder intervention consisting of a letter from the primary care physician, self-care handbook, preventive care checklist and specific recommendations regarding receipt of routine monitoring and screening

Comparator ( $\mathbf{n}=\mathbf{1 6 6 8})$ : usual care (not specified)

Duration: 12 months

Outcomes Primary outcome: documented receipt of fasting lipid profile, HbAlc measurement, dilated retinal exam during the period 6 - 12 months following randomisation

Secondary outcomes: $\mathrm{HbA} 1 \mathrm{c}$ and cholesterol levels $1 \mathrm{yr}$ after randomisation

Baseline screening attendance (control group): $47.1 \%$

\begin{tabular}{|c|c|c|}
\hline Notes & \multicolumn{2}{|c|}{$\begin{array}{l}\text { Date conducted: } 1999 \\
\text { Trial registration number: NR } \\
\text { Sources of funding: NR } \\
\text { Declaration of interest: NR }\end{array}$} \\
\hline \multicolumn{3}{|l|}{ Risk of bias } \\
\hline Bias & Authors' judgement & Support for judgement \\
\hline $\begin{array}{l}\text { Adequate sequence gener- } \\
\text { ation? }\end{array}$ & Low risk & $\begin{array}{l}\text { Quote: "Using the random number generator In SAS (Version 8.2: SAS Institute, } \\
\text { Inc.,Cary, NC) each month, each eligible patient with a birthday on the month } \\
\text { was assigned to receive either the mailed reminder packet or usual care." p } \\
522\end{array}$ \\
\hline $\begin{array}{l}\text { Adequate allocation con- } \\
\text { cealement? }\end{array}$ & Unclear risk & Not reported \\
\hline $\begin{array}{l}\text { Similar baseline outcome } \\
\text { measurements? }\end{array}$ & Low risk & $\begin{array}{l}\text { Judgement comment: baseline retinal exams reported and balanced across } \\
\text { study arms (Table } 2 \text { p 527) }\end{array}$ \\
\hline $\begin{array}{l}\text { Similar baseline character- } \\
\text { istics? }\end{array}$ & Low risk & $\begin{array}{l}\text { Quote: "Almost } 60 \% \text { of the study population received an } \mathrm{HbA} 1 \mathrm{c} \text { in the } 6 \\
\text { months preceding the mailed reminder program, and approximately half re- } \\
\text { ceived a lipid profile and a retinal exam in the } 12 \text { months preceding the mailed } \\
\text { reminder program, We found no statistically significant differences in these } \\
\text { and other characteristics listed in Table } 2 \text { between patients randomized to re- } \\
\text { ceive the mailed reminder program or usual care." p } 526\end{array}$ \\
\hline $\begin{array}{l}\text { Incomplete outcome data } \\
\text { addressed? }\end{array}$ & Low risk & Judgement comment: no missing outcome data (see Table 3 p 528) \\
\hline $\begin{array}{l}\text { Knowledge of allocated in- } \\
\text { tervention prevented? }\end{array}$ & Low risk & $\begin{array}{l}\text { Judgement comment: outcomes were obtained from automated clinical ad- } \\
\text { ministrative databases }\end{array}$ \\
\hline
\end{tabular}


Lafata 2002 (Continued)

Protected against contam- Low risk_ Judgement comment: it is unlikely that the control group received the mailed ination? intervention

\begin{tabular}{lll}
\hline $\begin{array}{l}\text { Free from selective out- } \\
\text { come reporting? }\end{array}$ & Unclear risk & $\begin{array}{l}\text { Judgement comment: trial retrospectively registered and therefore not possi- } \\
\text { ble to assess }\end{array}$ \\
\hline Other risks of bias? & Low risk & Judgement comment: no evidence of other risks of bias \\
\hline
\end{tabular}

\section{Lian 2013}

Study aim: to assess whether a small co-payment would impact on uptake of diabetic retinopthy
screening compared to free access

Study design: parallel-group RCT

Participants
Setting: 2 public family medicine clinics
Total number of patients: 4644
Percentage male: $45.2 \%$
Diabetes type: type 1 and 2
Average age (SD): 64.1 yrs (11)
Inclusion criteria: patients with type 1 or type 2 diabetes
Exclusion criteria: patients already under the regular care of an ophthalmologist
Intervention ( $\mathbf{n}=\mathbf{2 3 1 9}$ ): participants offered screening with small co-payment. A postal reminder of
the appointment was sent to those who accepted screening. Participants not attending for screening
were called to book a further appointment
Comparator ( $\mathbf{n}=\mathbf{2 3 2 5}$ : participants offered screening with no charge. A postal reminder of the ap-
pointment was sent to those who accepted screening. Participants not attending for screening were
called to book a further appointment
Duration: NR

Outcomes

Primary outcome: uptake of screening and severity of diabetic retinopathy detected

Secondary outcomes: NR

Baseline screening attendance (control group): NR

\begin{tabular}{ll}
\hline Notes & Date conducted: NR \\
& Trial registration number: NR
\end{tabular}

Sources of funding: Health and Health Services Research Fund of the Hong Kong SAR Government and the Azalea Endowment Fund.

Declaration of interest: none declared

\section{Risk of bias}

Bias Authors' judgement Support for judgement


Lian 2013 (Continued)

$\begin{array}{ll}\begin{array}{l}\text { Adequate sequence gener- } \\ \text { ation? }\end{array} & \text { Qow risk } \\ \text { computer.." p } 1248\end{array}$
ation? computer.." p 1248

Adequate allocation con- Low risk

Quote: " ..a research assistant generated the random sequence and assigned cealement? the participants...Two trained and experienced telephone interviewers were each allocated a random half of the subjects allocated to the free and pay groups." p 1248

Similar baseline outcome Unclear risk Not reported
measurements?

\begin{tabular}{|c|c|c|}
\hline $\begin{array}{l}\text { Similar baseline character- } \\
\text { istics? }\end{array}$ & Low risk & $\begin{array}{l}\text { Quote: "There were no differences between the characteristics of participants } \\
\text { allocated to the free and pay groups (Table 1)." p } 1248\end{array}$ \\
\hline
\end{tabular}
istics?

Judgement comment: the majority of exclusions were due to participants alIncomplete outcome data Low risk addressed? ready being under ophthalmologist care. Low attrition with reasons given and balanced across both arms of the study

Knowledge of allocated in- Unclear risk Not reported
tervention prevented?

Protected against contam- Unclear risk ination?
Quote: "Two trained and experienced telephone interviewers were each allocated a random half of the subjects allocated to the free and pay groups." $p$ 1248

Judgement comment: not clear how contamination was prevented

\begin{tabular}{lll}
\hline $\begin{array}{l}\text { Free from selective out- } \\
\text { come reporting? }\end{array}$ & Unclear risk & $\begin{array}{l}\text { Judgement comment: trial retrospectively registered and therefore not possi- } \\
\text { ble to assess }\end{array}$ \\
\hline Other risks of bias? & Low risk & Judgement comment: no evidence of other sources of bias \\
\hline
\end{tabular}

\section{Litaker 2003}

Methods Study aim: to compare a traditional physician-only model of care with a more collaborative, team-
based approach to chronic disease management

Study design: parallel-group RCT

\section{Participants}

\section{Country: USA}

Setting: Department of General Internal Medicine at the Cleveland Clinic Foundation, Ohio

Total number of participants: 157

Percentage male: $41 \%$

Diabetes type: type 2

Average age (SD): $60.5 \mathrm{yrs}(9)$

Inclusion criteria: patients with established diagnoses of mild or moderate hypertension and non-insulin-dependent diabetes mellitus without known end-organ complications

Exclusion criteria: medically complex individuals (Charlson index $>5$ ) or those requiring $3+$ medications for blood pressure control 
Litaker 2003 (Continued)

Interventions
Intervention ( $\mathbf{n}=\mathbf{7 9}$ ): clinical practice algorithms, patient education on disease self-management strategies, and regular monitoring and feedback delivered primarily by a nurse practitioner. The nurse practitioner acted as the first-line contact for care, in treatment decisions and to standardise treatment and for assessing treatment adherence and individual barriers to adherence

Comparator ( $\mathbf{n}=\mathbf{7 8}$ ): physician-only or 'usual' care defined as any form of treatment offered by an individual's primary care physician that reflected the practice style prevalent at the study site prior to the current investigation

Duration: 12 months
Primary outcome: measures to reflect the process and quality of care; documented evidence of annual ophthalmologic and foot examinations; HbA1c assessment at least once during the study year (other than study measures at 0 and 12 months); documentation of influenza and pneumococcal vaccination status and administration when appropriate

\section{Secondary outcomes: NR}

Baseline screening attendance (control group): NR

Trial registration number: NR

Sources of funding: Arison Foundation and the I.H. Page Center for Health Outcomes Research at the Cleveland Clinic Foundation

Declaration of interest: NR

\section{Risk of bias}

\begin{tabular}{|c|c|c|}
\hline Bias & Authors' judgement & Support for judgement \\
\hline $\begin{array}{l}\text { Adequate sequence gener- } \\
\text { ation? }\end{array}$ & Unclear risk & Not reported \\
\hline $\begin{array}{l}\text { Adequate allocation con- } \\
\text { cealement? }\end{array}$ & Unclear risk & Not reported \\
\hline $\begin{array}{l}\text { Similar baseline outcome } \\
\text { measurements? }\end{array}$ & Unclear risk & Not reported \\
\hline $\begin{array}{l}\text { Similar baseline character- } \\
\text { istics? }\end{array}$ & Low risk & $\begin{array}{l}\text { Quote: "Members of the two patient groups did not differ significantly at study } \\
\text { entry with respect to age, gender or racial composition, years of education } \\
\text { completed, number of comorbid conditions, or baseline HbAlc and blood } \\
\text { pressure control, total cholesterol or HDL-c values." p } 229\end{array}$ \\
\hline $\begin{array}{l}\text { Incomplete outcome data } \\
\text { addressed? }\end{array}$ & Low risk & Judgement comment: outcome on all participants randomised were reported \\
\hline $\begin{array}{l}\text { Knowledge of allocated in- } \\
\text { tervention prevented? }\end{array}$ & Unclear risk & Not reported \\
\hline $\begin{array}{l}\text { Protected against contam- } \\
\text { ination? }\end{array}$ & Low risk & $\begin{array}{l}\text { Quote: "Routine use of reminder systems, forms to facilitate documentation of } \\
\text { care, monitored use of clinical guidelines or active collaboration with a nurse } \\
\text { practitioner were not aspects of usual care for physicians in this practice dur- } \\
\text { ing the study period." }\end{array}$ \\
\hline & & p 226 \\
\hline
\end{tabular}


Litaker 2003 (Continued)

$\begin{array}{ll}\begin{array}{l}\text { Free from selective out- Unclear risk } \\ \text { come reporting? }\end{array} & \text { Judgement comment: no protocol or trial registry entry available and there- } \\ \end{array}$
come reporting? fore not possible to assess

Other risks of bias?

Study aim: to evaluate an intensive telephone follow-up as an additional component of a diabetes disease management programme already shown to be effective in improving glycaemic control, adherence with ADA standards of care, and HRQOL

Study design: parallel-group RCT

Setting: acute care teaching hospital

Total number of participants: 336

Percentage male: $46.7 \%$

Diabetes type: type 1 and 2

Average age (SD): 58 yrs (12.7)

Inclusion criteria: adult patients with type 1 or type 2 diabetes mellitus who were referred to the hospital-based disease management programme

\section{Exclusion criteria: NR}

Intervention ( $\mathbf{n}=\mathbf{1 7 6}$ ): both the intervention and control groups received the standard of care provided in the diabetes disease management programme as follows: (1) $3 \times 4$-hour educational classes covering topics such as living with diabetes, introduction to diabetes and the metabolic syndrome, nutrition and exercise, the importance of adherence to the ADA standards of care (e.g. annual eye exams, foot exams, blood glucose monitoring) and strategies to enhance self-management skills; (2) individual visits with a Registered Nurse and a nutritionist; (3) collaborative care management with written evaluations and recommendations provided to the participant's primary care provider, and scheduled follow-up visits. The intervention group also received a series of 12 weekly phone calls to reinforce education and self-management skills. The first call was $15-20 \mathrm{~min}$ in length; subsequent calls were $5-7$ minutes each

Comparator $(\mathbf{n}=\mathbf{1 6 0})$ : usual care consisting of the diabetes disease management programme as defined above, without the intensive telephone intervention

Duration: 12 months

Outcomes

Primary outcome: glycaemic control; general and disease-specific HRQOL; symptoms of depression; adherence to self-management guidelines, and participant satisfaction

\section{Secondary outcomes: NR}

Baseline screening attendance (control group): NR

Trial registration number: NR

Sources of funding: Aetna Quality of Care Research Foundation through the Academic Medicine and Managed Care Forum 
Maljanian 2005 (Continued)

Declaration of interest: NR

\section{Risk of bias}

\begin{tabular}{|c|c|c|}
\hline Bias & Authors' judgement & Support for judgement \\
\hline $\begin{array}{l}\text { Adequate sequence gener- } \\
\text { ation? }\end{array}$ & Unclear risk & Not reported \\
\hline $\begin{array}{l}\text { Adequate allocation con- } \\
\text { cealement? }\end{array}$ & Unclear risk & Not reported \\
\hline $\begin{array}{l}\text { Similar baseline outcome } \\
\text { measurements? }\end{array}$ & Unclear risk & Not reported \\
\hline $\begin{array}{l}\text { Similar baseline character- } \\
\text { istics? }\end{array}$ & High risk & $\begin{array}{l}\text { Quote: "A comparison of demographic and baseline measures indicated that } \\
\text { the two groups differed on age, BMI, when diagnosed, language used in the } \\
\text { DLC class attended, ethnicity (Caucasian, non-Caucasian dichotomy), HbA1c, } \\
\text { PCS, MCS, and symptoms of depression (CES-D)." p } 18 \\
\text { Judgement comment: the reported baseline imbalance could have influenced } \\
\text { retinopathy screening attendance }\end{array}$ \\
\hline $\begin{array}{l}\text { Incomplete outcome data } \\
\text { addressed? }\end{array}$ & High risk & $\begin{array}{l}\text { Quote: "The } 171 \text { participants who did not return for their two follow-up visits } \\
\text { represent a significant attrition rate (34\%)." p } 18 \\
\text { Quote: "The fact that individuals with better glycemic control were more likely } \\
\text { to return may explain some of the floor effect on glycemic control in the total } \\
\text { study population. Further, that those patients with worse glycemic control and } \\
\text { larger BMI at enrollment were the ones more likely to miss later appointments } \\
\text { is concerning because those are the patients who most need their diabetes ed- } \\
\text { ucation reinforced and self-management encouraged." p } 23\end{array}$ \\
\hline
\end{tabular}

Knowledge of allocated in- Unclear risk Not reported
tervention prevented?

tervention prevented?

Judgement comment: unlikely that control group received the intervention

\begin{tabular}{lll}
$\begin{array}{l}\text { Protected against contam- } \\
\text { ination? }\end{array}$ & Low risk & Judgement comment: unlikely that control group received the intervention \\
\hline $\begin{array}{l}\text { Free from selective out- } \\
\text { come reporting? }\end{array}$ & Unclear risk & $\begin{array}{l}\text { Judgement comment: no protocol or trial registry entry available and there- } \\
\text { fore not possible to assess }\end{array}$
\end{tabular}

Other risks of bias? Low risk Judgement comment: no evidence of other sources of bias
examinations compared with traditional surveillance in community health clinics with a high proportion of ethnic minorities

Study design: parallel-group RCT

\section{Participants}

\section{Country: USA}

Setting: 2 community health clinics

Total number of participants: 567 
Mansberger 2015 (Continued)

\section{Percentage male: $48 \%$}

Diabetes type: NR

Average age (SD): 51.1 yrs (11.8)

Inclusion criteria: diabetic patients $\geq 18$ years with diabeted who were scheduled to visit their primary care provider

Exclusion criteria: cognitive impairment preventing informed consent; inability to transfer to a chair to perform non-mydriatic imaging

Interventions

Intervention ( $\mathbf{n}=\mathbf{2 9 6}$ ): participants in this group had digital images of their retina captured with a non-mydriatic camera and were encouraged to see an eye care provider annually for a diabetic eye exam

Comparator $(\mathbf{n}=\mathbf{2 7 1})$ : participants in this group were encouraged to see an eye care provider annually for a diabetic eye exam

Duration: 48 months (intervention offered to comparator group after 18 months)

Outcomes

Primary outcome: proportion of participants that receive an annual eye exam

Secondary outcomes: health belief factors associated with adherence

Baseline screening attendance (control group): NR

Notes

Date conducted: 1 August 2006 to 31 September 2009

Trial registration number: NCT01364129

Sources of funding: National Eye Institute; Centers for Disease Control and Prevention; Good Samaritan Foundation at Legacy Health

Declaration of interest: none declared

\section{Risk of bias}

\begin{tabular}{lll}
\hline Bias & Authors' judgement & Support for judgement \\
\hline $\begin{array}{l}\text { Adequate sequence gener- } \\
\text { ation? }\end{array}$ & Low risk & $\begin{array}{l}\text { Quote: "We used a random number generator to randomly assign participants } \\
\text { to the telemedicine group or the traditional surveillance group." p 519 }\end{array}$ \\
\hline $\begin{array}{l}\text { Adequate allocation con- } \\
\text { cealement? }\end{array}$ & Unclear risk & Not reported \\
\hline $\begin{array}{l}\text { Similar baseline outcome } \\
\text { measurements? }\end{array}$ & Unclear risk & Not reported \\
\hline
\end{tabular}

Similar baseline character- Low risk Quote: "There were no differences in demographic and medical characteristics istics? at enrolment between the telemedicine $(n=296)$ and traditional surveillance $(\mathrm{n}=271)$ groups." p 521

\begin{tabular}{ll}
\hline $\begin{array}{l}\text { Incomplete outcome data } \\
\text { addressed? }\end{array}$ & Low risk \\
& CONSORT flow diagram $\mathrm{p} \mathrm{519)}$
\end{tabular}

Knowledge of allocated in- Unclear risk Not reported
tervention prevented?

$\begin{array}{ll}\begin{array}{l}\text { Protected against contam- Low risk } \\ \text { ination? }\end{array} & \begin{array}{l}\text { Judgement comment: it is unlikely that the control group received the } \\ \text { telemedicine intervention }\end{array}\end{array}$


Mansberger 2015 (Continued)

Free from selective out- Unclear risk Judgement comment: trial retrospectively registered and so not possible to come reporting? assess

Other risks of bias?

Low risk

Judgement comment: no evidence of other risks of bias

McCall 2011

Methods Study aim: to evaluate the impact of commercial programmes for disease management that use nurse-based call centres on the quality of clinical care, acute care utilisation, and Medicare expenditures for Medicare fee-for-service beneficiaries

Study design: parallel-group RCT

Participants

Country: USA

Setting: primary care practices

Total number of participants: 188,169 patients with diabetes

Percentage male: NR

Diabetes type: NR

Average age (SD): NR

Inclusion criteria: Medicare beneficiaries in each of 8 geographic areas who met the selection criteria for heart failure or diabetes and had a HCC risk score of 1.35

Exclusion criteria: NR

Intervention ( $n=126,557$ participants with diabetes alone or diabetes and heart failure): Medicare Health Support Pilot Program consisting of 8 commercial programmes for disease management that used nurse-based call centres to assess the needs of individual beneficiaries and used health coaches to target those beneficiaries at immediate high risk for adverse events. The goals of the intervention were to improve beneficiaries' understanding of their disease or diseases, their ability to manage selfcare, and their ability to communicate with providers. Various educational resources including literature, videos, and Internet resources were provided. A small portion of the intervention population received intensive case-management services.

Comparator ( $n=61,612$ participants with diabetes alone or diabetes and heart failure): usual care (not specified)

Duration: 12 months

Outcomes

Primary outcome: changes from baseline compared between the intervention and control groups for the quality of clinical care provided, the use of acute care, and Medicare expenditures. 4 annual evidence-based processes of care measures were evaluated for patients with diabetes: glycated haemoglobin testing, urinary protein screening, retinal examination and LDL cholesterol testing.

Secondary outcomes: none

Baseline screening attendance (control group): $36.1 \%$

Notes

Date conducted: 2004 to 2007

Trial registration number: NR

Sources of funding: NR

Declaration of interest: none declared 
McCall 2011 (Continued)

Outcome data (based on pooled rates per 100 beneficiaries) calculated from Supplementary Table 1

(supplementary appendix) using the \% of participants with diabetes given in Table 1 (Main report).

\section{Risk of bias}

\begin{tabular}{|c|c|c|}
\hline Bias & Authors' judgement & Support for judgement \\
\hline $\begin{array}{l}\text { Adequate sequence gener- } \\
\text { ation? }\end{array}$ & Unclear risk & Not reported \\
\hline $\begin{array}{l}\text { Adequate allocation con- } \\
\text { cealement? }\end{array}$ & Unclear risk & Not reported \\
\hline $\begin{array}{l}\text { Similar baseline outcome } \\
\text { measurements? }\end{array}$ & Low risk & $\begin{array}{l}\text { Judgement comment: similar baseline screening attendance (see Table 1. On- } \\
\text { line supplement) }\end{array}$ \\
\hline $\begin{array}{l}\text { Similar baseline character- } \\
\text { istics? }\end{array}$ & Low risk & $\begin{array}{l}\text { Quote: "The characteristics of the beneficiaries were well balanced between } \\
\text { the intervention and control groups at baseline (Table 1)." p } 1707\end{array}$ \\
\hline $\begin{array}{l}\text { Incomplete outcome data } \\
\text { addressed? }\end{array}$ & Unclear risk & Not reported \\
\hline $\begin{array}{l}\text { Knowledge of allocated in- } \\
\text { tervention prevented? }\end{array}$ & Low risk & $\begin{array}{l}\text { Judgement comment: data on retinopathy screening obtained from routine- } \\
\text { ly-collected data }\end{array}$ \\
\hline $\begin{array}{l}\text { Protected against contam- } \\
\text { ination? }\end{array}$ & Low risk & $\begin{array}{l}\text { Judgement comment: it is unlikely that the control group received the } \\
\text { Medicare Health Support Programme }\end{array}$ \\
\hline $\begin{array}{l}\text { Free from selective out- } \\
\text { come reporting? }\end{array}$ & Unclear risk & $\begin{array}{l}\text { Judgement comment: no protocol or trial registry entry available and there- } \\
\text { fore not possible to assess }\end{array}$ \\
\hline Other risks of bias? & Low risk & Judgement comment: no evidence of other risks of bias \\
\hline
\end{tabular}

\section{McClellan 2003}

Methods Study aim: to determine if an intervention that includes claims-based feedback about patterns of $\mathrm{HbA1c}$ measurement results in more frequent monitoring of HbAlc in diabetic Medicare beneficiaries

Study design: cluster-RCT

\section{Participants}

Country: USA

Setting: primary care physicians in a southern state treating Medicare beneficiaries

Number of clusters: 123

Number of providers: 477

Total number of patients: 22,971

Percentage male: $43 \%$

Diabetes type: type 1 and type 2

Average age (SD): 74 yrs (NR)

Inclusion criteria: diabetes diagnosis based on 2 outpatient claims 30 days apart or 1 inpatient claim for the care of diabetes mellitus (250.xx, 357.2x, 362.0x, 366.41). Patients had to be aged at least 65, enrolled in Medicare for a minimum of 11 months in 1996 or 1998 
Interventions Intervention (63 clusters, $\mathbf{n}=\mathbf{1 1 , 9 0 4}$ participants): mailing to physicians at baseline, 2 months, 4 months, and 6 months containing clinical practice guidelines, general information about patterns of diabetes care in the state, an educational tape, and practice aids to implement guideline recommendations (chart stickers, pocket guides, wall posters, etc.). Intervention physicians were provided with fliers to remind participants to have regular check-ups of their urine, eyes, feet, and blood; an ADA catalogue containing diabetes-related publications and patient education presentations; and a 'Diabetic Passport' that allowed a patient to record their diabetic test results. The passport displayed the ADA recommendations for $\mathrm{HbAlc}$, eye, urine, and lipid monitoring

Comparator (61 clusters, $\mathbf{n}=\mathbf{1 1 , 0 6 7}$ participants): newsletter sent to intervention and comparator groups containing an article devoted to early detection of microvascular complication and the importance of glycaemic control which opened up to create a poster showing the tests/screenings that patients with diabetes mellitus require on a regular basis

Duration: 6 months

Outcomes Primary outcome: changes in frequency of measurement of HbAlc, quantitative urine protein and di-
lated eye examinations

Secondary outcomes: NR

Baseline screening attendance (control group): $39.3 \%$

\begin{tabular}{ll}
\hline Notes & Date conducted: 1996 to 1998 \\
& Trial registration number: NR \\
& Sources of funding: NR \\
& Declaration of interest: NR
\end{tabular}

\section{Risk of bias}

\begin{tabular}{|c|c|c|}
\hline Bias & Authors' judgement & Support for judgement \\
\hline $\begin{array}{l}\text { Adequate sequence gener- } \\
\text { ation? }\end{array}$ & Low risk & $\begin{array}{l}\text { Quote: "After assigning patients to physicians and physicians to counties, the } \\
\text { counties were ordered alphabetically and a random number table was used to } \\
\text { assign a county to either the intervention or comparison group." p } 1212\end{array}$ \\
\hline $\begin{array}{l}\text { Adequate allocation con- } \\
\text { cealement? }\end{array}$ & Low risk & $\begin{array}{l}\text { Quote: "None of the staff involved with the design and implementation of the } \\
\text { intervention were involved with the randomization of counties or selection of } \\
\text { physicians within counties." p } 1212\end{array}$ \\
\hline $\begin{array}{l}\text { Similar baseline outcome } \\
\text { measurements? }\end{array}$ & Low risk & $\begin{array}{l}\text { Judgement comment: similar proportion of baseline eye exams (see Table } 2 \mathrm{p} \\
\text { 1214) }\end{array}$ \\
\hline \multirow[t]{2}{*}{$\begin{array}{l}\text { Similar baseline character- } \\
\text { istics? }\end{array}$} & Low risk & $\begin{array}{l}\text { Quote: "The two groups were comparable with respect to race, gender, and } \\
\text { the mean age of the diabetic." p } 1213 \text { (see also Table } 1 \text { p 1214) }\end{array}$ \\
\hline & & $\begin{array}{l}\text { Judgement comment: Similar quality indicators at baseline (see Table } 2 \text { p } \\
\text { 1214) }\end{array}$ \\
\hline $\begin{array}{l}\text { Incomplete outcome data } \\
\text { addressed? }\end{array}$ & Low risk & $\begin{array}{l}\text { Quote: "...the dropout rate among practices in the comparison and interven- } \\
\text { tion groups was small, } 3.6 \text { and } 3.0 \% \text {, respectively, and thus was unlikely to bias } \\
\text { our results." p } 1215\end{array}$ \\
\hline $\begin{array}{l}\text { Knowledge of allocated in- } \\
\text { tervention prevented? }\end{array}$ & Low risk & $\begin{array}{l}\text { Judgement comment: eye-screening outcomes obtained from routinely-col- } \\
\text { lected claims data }\end{array}$ \\
\hline
\end{tabular}


McClellan 2003 (Continued)

Protected against contam- Low risk Judgement comment: control group unlikely to have received the intervention ination?

\begin{tabular}{|c|c|c|}
\hline $\begin{array}{l}\text { Free from selective out- } \\
\text { come reporting? }\end{array}$ & Unclear risk & $\begin{array}{l}\text { Judgement comment: no protocol or trial registry entry available and there- } \\
\text { fore not possible to assess }\end{array}$ \\
\hline
\end{tabular}

Other risks of bias? Low risk Judgement comment: no evidence of other sources of bias

\section{McDermott 2001}

Methods Study aim: to evaluate a paper-based recall and reminder system and basic diabetes education of healthcare workers in improving the quality of diabetes care in a remote indigenous community

Study design: cluster-RCT

\section{Participants}

Country: Australia

Setting: 21 primary health care centres in Torres Strait and Northern Peninsula Area in Queensland Australia

\section{Number of clusters: 21}

Number of providers: 3

Total number of patients: 555

Percentage male: $38 \%$

Diabetes type: NR

Average age (SD): $52.3 \mathrm{yrs}(13.5)$

Inclusion criteria: patients with diabetes

Exclusion criteria: patients aged $<15$ years diagnosed $<1$ year before the audit

Intervention (8 clusters, $\mathbf{n}=\mathbf{2 5 0}$ participants)): intervention and comparator sites received audit and feedback on patients with diabetes benchmarked against guidelines. Evidence-based guidelines were issued and a new diabetes outreach service was established (comprising a diabetologist, nutritionist, podiatrist, and diabetes healthcare worker). Intervention and comparator sites were visited by the outreach team who saw individual patients on a referral basis. A recall system was established in intervention sites and healthcare workers in these sites received clinical training on the basics of diabetes care

Comparator (13 clusters, $\mathbf{n}=\mathbf{3 0 5}$ participants): see above

Duration: 12 months

Outcomes

Primary outcome: proportion of participants fulfilling diabetes care indicators (including 'eye check' or 'ophthalmologist check') in the last 12 months

Secondary outcomes: diabetes-related hospital admissions and hospitalisations

Baseline screening attendance (control group): $29.8 \%$

Notes

Date conducted: March 1999 to February 2000

Trial registration number: NR

Sources of funding: National Health and Medical Research Council 
McDermott 2001 (Continued)

Declaration of interest: NR

\title{
Risk of bias
}

\begin{tabular}{|c|c|c|}
\hline Bias & Authors' judgement & Support for judgement \\
\hline \multirow[t]{2}{*}{$\begin{array}{l}\text { Adequate sequence gener- } \\
\text { ation? }\end{array}$} & High risk & $\begin{array}{l}\text { Quote: "..eight intervention sites were chosen randomly by being picked from a } \\
\text { hat containing the names of all } 21 \text { clinics" } \mathrm{p} 498\end{array}$ \\
\hline & & Judgement comment: inappropriate method of sequence generation \\
\hline $\begin{array}{l}\text { Adequate allocation con- } \\
\text { cealement? }\end{array}$ & Low risk & $\begin{array}{l}\text { Judgement comment: unit of allocation by primary care practice and alloca- } \\
\text { tion performed prior to the start of the study }\end{array}$ \\
\hline $\begin{array}{l}\text { Similar baseline outcome } \\
\text { measurements? }\end{array}$ & Low risk & $\begin{array}{l}\text { Judgement comment: similar rates of eye checks and ophthalmology visits at } \\
\text { baseline }\end{array}$ \\
\hline \multirow[t]{2}{*}{$\begin{array}{l}\text { Similar baseline character- } \\
\text { istics? }\end{array}$} & Low risk & $\begin{array}{l}\text { Quote: "There were no significant differences in age, sex ratio and duration of } \\
\text { diabetes at baseline..." p } 498\end{array}$ \\
\hline & & $\begin{array}{l}\text { Judgement comment; baseline differences between arms in diabetes process- } \\
\text { es of care (Table } 2 \text { p 499) but unlikely to influence outcome }\end{array}$ \\
\hline
\end{tabular}

Incomplete outcome data Low risk Judgement comment: low attrition and balanced across arms

addressed?

Knowledge of allocated in- Unclear risk Not reported

tervention prevented?

\begin{tabular}{lll}
$\begin{array}{l}\text { Protected against contam- } \\
\text { ination? }\end{array}$ & Low risk & Judgement comment: control group unlikely to have received the intervention \\
\hline $\begin{array}{l}\text { Free from selective out- } \\
\text { come reporting? }\end{array}$ & Unclear risk & $\begin{array}{l}\text { Judgement comment: no protocol or trial registry entry available and there- } \\
\text { fore not possible to assess }\end{array}$
\end{tabular}

Other risks of bias? Low risk Judgement comment: no evidence of other sources of bias

\section{Meigs 2003}

Methods Study aim: to evaluate effects of a web-based decision-support tool, the diabetes 'Disease Management Application (DMA)', to improve evidence-based management of type 2 diabetes

Study design: cluster-RCT

Participants

\author{
Country: USA \\ Number of clusters: 26 \\ Number of providers: 26 \\ Total number of patients: 598 \\ Percentage male: $48.1 \%$ \\ Diabetes type: type 2 \\ Average age (SD): 67.5 yrs (12)
}

Setting: Adult Medicine Clinic (AMC) in Harvard Medical School in Boston Massachusetts USA 
Meigs 2003 (Continued)

Inclusion criteria: patients with at least 1 visit to the AMC during the pre-intervention year (May 1997 to April 1998) were identified by billing claims, and patients with type 2 diabetes were identified by ICD-9 codes $250.00-250.90$

Exclusion criteria: type 1 diabetes

$\begin{array}{ll}\text { Interventions } & \text { Intervention (12 clusters, } \mathbf{n}=\mathbf{3 0 7} \text { participants): web-based information management/clinical deci- } \\ \text { sion-support tool providing a single-screen view of patient-specific information, enabling decision sup- } \\ \text { port at the time of patient contact. The decision-support tool generated patient-specific recommenda- } \\ \text { tions based on evidence-based guidelines }\end{array}$

Comparator (14 clusters, $\mathbf{n}$ = 291 participants): usual care (not specified)

Duration: 12 months

Outcomes

Primary outcome: change in rates of annual $\mathrm{HbA1c}, \mathrm{LDL}$ cholesterol, BP, and eye and foot screening and change in the absolute values of $\mathrm{HbA1c}$, LDL cholesterol, and blood pressure

Secondary outcomes: NR

Baseline screening attendance (control group): $41.2 \%$

\begin{tabular}{ll}
\hline Notes & Date conducted: May 1998 to April 1999 \\
& Trial registration number: NR \\
& Sources of funding: National Pharmaceutical Council; MGH Primary Care Operations Improvement \\
& and Clinical Research Programs
\end{tabular}

Declaration of interest: NR

\begin{tabular}{|c|c|c|}
\hline \multicolumn{3}{|l|}{ Risk of bias } \\
\hline Bias & Authors' judgement & Support for judgement \\
\hline $\begin{array}{l}\text { Adequate sequence gener- } \\
\text { ation? }\end{array}$ & Low risk & $\begin{array}{l}\text { Quote: "A coin was tossed to select an intervention group and a control group." } \\
\text { p } 751\end{array}$ \\
\hline $\begin{array}{l}\text { Adequate allocation con- } \\
\text { cealement? }\end{array}$ & Low risk & $\begin{array}{l}\text { Judgement comment: unit of allocation by primary care practice and alloca- } \\
\text { tion performed prior to the start of the study }\end{array}$ \\
\hline $\begin{array}{l}\text { Similar baseline outcome } \\
\text { measurements? }\end{array}$ & High risk & $\begin{array}{l}\text { Quote: "..rates of eye and foot screening were lower in the intervention group." } \\
\text { p } 793\end{array}$ \\
\hline & & Judgement comment: baseline imbalance in diabetic retinopathy screening \\
\hline $\begin{array}{l}\text { Similar baseline character- } \\
\text { istics? }\end{array}$ & Low risk & $\begin{array}{l}\text { Quote: "Baseline staff provider and patient characteristics were similar com- } \\
\text { paring the intervention group with the control group (Table 1)." p } 793\end{array}$ \\
\hline $\begin{array}{l}\text { Incomplete outcome data } \\
\text { addressed? }\end{array}$ & Low risk & Judgement comment: data from all participants reported \\
\hline $\begin{array}{l}\text { Knowledge of allocated in- } \\
\text { tervention prevented? }\end{array}$ & Low risk & $\begin{array}{l}\text { Quote: "Clinical data from paper and electronic charts were abstracted by } \\
\text { three nurses blinded to group status of providers and patients." p } 752\end{array}$ \\
\hline $\begin{array}{l}\text { Protected against contam- } \\
\text { ination? }\end{array}$ & Low risk & Judgement comment: control group unlikely to have received the intervention \\
\hline $\begin{array}{l}\text { Free from selective out- } \\
\text { come reporting? }\end{array}$ & Unclear risk & $\begin{array}{l}\text { Judgement comment: no protocol or trial registry entry available and there- } \\
\text { fore not possible to assess }\end{array}$ \\
\hline
\end{tabular}


Meigs 2003 (Continued)

Other risks of bias? Low risk Judgement comment: no evidence of other sources of bias

O'Connor 2005

Methods Study aim: to evaluate the impact of a QI intervention on the quality of diabetes care

Study design: cluster-RCT

\title{
Participants
}

\author{
Country: USA \\ Setting: primary care medical practices in Minnesota \\ Number of clusters: 12
}

Number of providers: 329

Total number of patients: 754

Percentage male: $54.3 \%$

Diabetes type: NR

Average age (SD): $57.8 \mathrm{yrs}(\mathrm{NR})$

Inclusion criteria: aged > 19 years who had 2+ ICD-9 diagnostic codes for diabetes in a defined 12month period

\section{Exclusion criteria: NR}

Intervention ( $\mathbf{6}$ clusters, $\mathbf{n}=\mathbf{4 2 8}$ participants): IDEAL (Improving Care for Diabetes Through Empowerment Active Collaboration and Leadership) model consisting of facilitation of leadership actions in support of change, training for the leader and facilitator of an intra-clinic multidisciplinary continuous quality improvement (CQI) team, and consultative and networking support of the change process

Comparator ( 6 clusters, $\mathbf{n}=\mathbf{3 2 6}$ participants): usual care (not specified)

Duration: 18 months

Outcomes

Primary outcome: $\%$ of participants with annual tests of HbA1c, LDL and BP; \% of participants with annual screening for foot, eye or kidney complications

Secondary outcomes: NR

Baseline screening attendance (control group): $39 \%$

\begin{tabular}{|c|c|c|}
\hline Notes & \multicolumn{2}{|c|}{$\begin{array}{l}\text { Sources of funding: Centres for Disease Control and Prevention; HealthPartners Research Foundation } \\
\text { Declaration of interest: } 1 \text { author reported being a member of advisory boards and receiving honoraria } \\
\text { from LifeScan, NovoNordisk and AmerisourceBergen }\end{array}$} \\
\hline \multicolumn{3}{|l|}{ Risk of bias } \\
\hline Bias & Authors' judgement & Support for judgement \\
\hline $\begin{array}{l}\text { Adequate sequence gener- } \\
\text { ation? }\end{array}$ & Unclear risk & Not reported \\
\hline
\end{tabular}


O'Connor 2005 (Continued) $\begin{array}{ll}\begin{array}{l}\text { Adequate allocation con- } \\ \text { cealement? }\end{array} & \text { Low risk } \\ \end{array}$

Similar baseline outcome Low risk

Judgement comment: similar attendance for annual eye exams at baseline measurements?

\section{Similar baseline character- Low risk istics?}

Quote: "Table 1 shows that the clinics and patients in the intervention and control group were similar in size and in patient mix..." p 1892

\section{Incomplete outcome data High risk} addressed?
Judgement comment: reported data was based on those 754 participants who completed the pre- and post-intervention surveys and consented to have their medical record reviewed. Response rates to the survey averaged 55\% - 65\% across study sites

Knowledge of allocated in- Unclear risk Not reported

tervention prevented?

\begin{tabular}{lll}
\hline $\begin{array}{l}\text { Protected against contam- } \\
\text { ination? }\end{array}$ & Low risk & Judgement comment: control group unlikely to have received the intervention \\
\hline $\begin{array}{l}\text { Free from selective out- } \\
\text { come reporting? }\end{array}$ & Unclear risk & $\begin{array}{l}\text { Judgement comment: no protocol or trial registry entry available and there- } \\
\text { fore not possible to assess }\end{array}$ \\
\hline
\end{tabular}

\begin{tabular}{ll}
\hline Other risks of bias? $\quad$ Low risk Judgement comment: no evidence of other sources of bias \\
\hline
\end{tabular}

\section{Perria 2007}

Study aim: to assess the effectiveness of different strategies for the implementation of an evi-
dence-based guideline for the management of non-complicated type 2 diabetes mellitus

Study design: cluster-RCT

Participants

Country: Italy

Setting: primary care setting of Italian National Health Service in Lazio region of Central Italy

Number of clusters: 252

Number of providers: 252

Total number of patients: 6290

Percentage male: $52 \%$

Diabetes type: type 2

Average age (SD): $65 \mathrm{yrs}(10)$

Inclusion criteria: patients with uncomplicated type 2 diabetes

Exclusion criteria: NR

Interventions

Intervention (active implementation)(84 clusters, $\mathbf{n}=1952$ participants): 2 -day training

module and consequent administration of a diabetes guideline

Intervention (passive implementation) (85 clusters, $\mathbf{n}=\mathbf{2 1 0 6}$ participants): GPs received the guideline without any training but with a written request to implement the guideline 
Perria 2007 (Continued)

Comparator (83 clusters, $\mathbf{n}=\mathbf{2 2 3 2}$ participants): usual care (not specified)

Duration: 1 month
Primary outcome: GPs' adherence to guideline recommendations for diabetes management (including proportion of participants who were prescribed all microvascular complications assessment tests: eye examination or fundus and blood creatinine or creatinine clearance and microalbuminuria) per year

Secondary outcomes: GPs' drug-prescribing behaviour

Baseline screening attendance (control group): $22.9 \%$

Trial registration number: ISRCTN80116232

Sources of funding: Italian Ministry of Health

Declaration of interest: None declared

Study protocol has been published: www.ncbi.nlm.nih.gov/pubmed/15196307

\section{Risk of bias}

\begin{tabular}{|c|c|c|}
\hline Bias & Authors' judgement & Support for judgement \\
\hline $\begin{array}{l}\text { Adequate sequence gener- } \\
\text { ation? }\end{array}$ & Low risk & $\begin{array}{l}\text { Quote: "Our randomisation sequences was computer-generated. GPs who ac- } \\
\text { cepted to take part in the study, were assigned by simple random allocation by } \\
\text { the REXSCO software..." p } 4\end{array}$ \\
\hline $\begin{array}{l}\text { Adequate allocation con- } \\
\text { cealement? }\end{array}$ & Low risk & $\begin{array}{l}\text { Quote: "Randomisation was performed by a researcher not involved in the } \\
\text { study and who was blind to the identity of the practices." p } 4\end{array}$ \\
\hline $\begin{array}{l}\text { Similar baseline outcome } \\
\text { measurements? }\end{array}$ & Low risk & $\begin{array}{l}\text { Judgement comment: similar retinal screening attendance at baseline (see Ta- } \\
\text { ble } 3 \text { p } 6 \text { ) }\end{array}$ \\
\hline $\begin{array}{l}\text { Similar baseline character- } \\
\text { istics? }\end{array}$ & Low risk & $\begin{array}{l}\text { Judgement comment: similar baseline demographic and clinical characteris- } \\
\text { tics }\end{array}$ \\
\hline $\begin{array}{l}\text { Incomplete outcome data } \\
\text { addressed? }\end{array}$ & High risk & $\begin{array}{l}\text { Judgement comment: high attrition and missing data not balanced across } \\
\text { study arms }\end{array}$ \\
\hline $\begin{array}{l}\text { Knowledge of allocated in- } \\
\text { tervention prevented? }\end{array}$ & Unclear risk & Not reported \\
\hline $\begin{array}{l}\text { Protected against contam- } \\
\text { ination? }\end{array}$ & Unclear risk & $\begin{array}{l}\text { Quote: "Our randomisation sequences was computer-generated. GPs who ac- } \\
\text { cepted to take part in the study, were assigned by simple random allocation by } \\
\text { the REXSCO software, which assigns to same-practice partners a nil probabil- } \\
\text { ity of being randomised, thus minimising the chances of participant contami- } \\
\text { nation." p } 4\end{array}$ \\
\hline $\begin{array}{l}\text { Free from selective out- } \\
\text { come reporting? }\end{array}$ & Low risk & $\begin{array}{l}\text { Judgement comment: reported outcomes consistent with trial registry } \\
\text { ISRCTN80116232 }\end{array}$ \\
\hline Other risks of bias? & High risk & Judgement comment: only $25 \%$ of eligible GPs agreed to take part \\
\hline
\end{tabular}


Peterson 2008

Methods Study aim: to determine whether implementation of a multicomponent organisational intervention can produce significant change in diabetes care and outcomes in community primary care practices

Study design: cluster-RCT

\section{Participants}

Country: USA

Setting: 24 community care practices in Minnesota

Number of clusters: 24

Number of providers: 238

Total number of patients: 7101

Percentage male: $50.3 \%$

Diabetes type: type 2

Average age (SD): $62.8 \mathrm{yrs}(0.9)$

Inclusion criteria: all type 2 diabetic patients in each practice aged 18 - 89 years

Exclusion criteria: documented as not receiving diabetes care at the practice (referred care); deceased; no longer in the practice (documented transfer or no contact or 24 months); permanently residing in a long-term care facility sisting of implementation of an electronic diabetes registry, visit reminders, and patient-specific physician alerts. A site co-ordinator facilitated pre-visit planning and a monthly review of performance with a local physician champion

Comparator (12 clusters, $\mathbf{n}=\mathbf{3 1 3 1}$ participants): usual care (practices were provided with a report of their process and outcome measures at baseline and were encouraged to continue usual quality improvement)

Duration: 12 months

Outcomes

Primary outcome: \% of participants achieving target values for the composite of SBP $<130 \mathrm{mmHg}$, $\mathrm{LDL}$ cholesterol $<100 \mathrm{mg} / \mathrm{dl}$, and $\mathrm{HbAlc}<7.0 \%$ at baseline and 12 months

Secondary outcomes: 6 diabetes care process measures (including annual eye examination)

Baseline screening attendance (control group): $24.8 \%$

Notes Date conducted: NR

Trial registration number: NCT00108927

Sources of funding: National Institute of Diabetes, Digestive, and Kidney Disorders, National Institutes of Health

\section{Declaration of interest: NR}

\section{Risk of bias}

\begin{tabular}{lll}
\hline Bias & Authors' judgement & Support for judgement \\
\hline $\begin{array}{l}\text { Adequate sequence gener- } \\
\text { ation? }\end{array}$ & Unclear risk & Not reported \\
\hline $\begin{array}{l}\text { Adequate allocation con- } \\
\text { cealement? }\end{array}$ & Low risk & $\begin{array}{l}\text { Quote: "Practices were randomized in blocks of four using six sets of opaque } \\
\text { envelopes to ensure that equal numbers of control and intervention clinics }\end{array}$ \\
\hline
\end{tabular}


Peterson 2008 (Continued)

were abstracted simultaneously. Envelopes were prepared by the statistician, assigned in order of postmark, and opened under observation." p 2239

Similar baseline outcome High risk
measurements?
measurements?
Judgement comment: higher attendance for eye examination in intervention clinics at baseline (35.5\% versus $24.8 \%$, Table 3 p 2241) and baseline imbalance in diabetic retinopathy (Table 2 p 2240)

Quote: "No statistically significant differences existed between intervention and control practices in patient demographics, total number of diabetes complications, or relevant clinical measures." p 2240

Judgement comment: with the exception diabetic retinopathy, all other baseline clinical characteristics were similar (Table 2 p 2240)

\begin{tabular}{lll}
\hline $\begin{array}{l}\text { Incomplete outcome data } \\
\text { addressed? }\end{array}$ & Low risk & Judgement comment: data from all participants included in the analysis \\
\hline $\begin{array}{l}\text { Knowledge of allocated in- } \\
\text { tervention prevented? }\end{array}$ & Unclear risk & Not reported \\
\hline $\begin{array}{l}\text { Protected against contam- } \\
\text { ination? }\end{array}$ & Low risk & Judgement comment: control group unlikely to have received the intervention \\
\hline $\begin{array}{l}\text { Free from selective out- } \\
\text { come reporting? }\end{array}$ & Low risk & $\begin{array}{l}\text { Judgement comment: reported outcomes consistent with trial registry } \\
\text { NCTO0108927 }\end{array}$ \\
\hline \begin{tabular}{l} 
Other risks of bias? \\
\hline
\end{tabular}
\end{tabular}

Piette 2001

Methods Study aim: to evaluated automated telephone disease management (ATDM) with telephone nurse follow-up as a strategy for improving diabetes treatment processes and outcomes in Department of Veterans Affairs (VA) clinics

Study design: parallel-group RCT

\section{Participants}

\section{Country: USA}

Setting: 4 university-affiliated VA clinics in northern California

Total number of participants: 292

Percentage male: $97 \%$

Diabetes type: NR

Average age (SD): 60.5 yrs (10)

Inclusion criteria: adults with a diagnosis of diabetes and an active prescription for a hypoglycaemic agent

Exclusion criteria: > 75 years of age; mentally ill; a life expectancy of $<12$ months; were newly diagnosed; planned to discontinue receiving services from the clinic within the 12-month follow-up period; did not have a touch-tone telephone

Interventions

Intervention ( $\mathbf{n}=\mathbf{1 4 6}$ ): bi-weekly automated telephone disease management (ATDM) health assessment and self-care education calls, and a nurse educator follow-up with participants based on their ATDM assessment reports 
Piette 2001 (Continued)

Comparator $(\mathbf{n}=\mathbf{1 4 6})$ : usual care (not specified)

Duration: 12 months

\begin{tabular}{ll}
\hline Outcomes & Primary outcome: impact on processes of care (including use of ophthalmology services); glycaemic \\
control
\end{tabular}

control

Secondary outcomes: participants' self-care activities and satisfaction with care

Baseline screening attendance (control group): $29.3 \%$

$\begin{array}{ll}\text { Notes } & \text { Date conducted: NR } \\ & \text { Trial registration number: NR } \\ & \text { Sources of funding: Health Services Research and Development Service, Mental Health Strategic } \\ & \text { Health Care Group, Quality Enhancement Research Initiative, Department of Veterans Affairs; American } \\ & \text { Diabetes Association }\end{array}$

Declaration of interest: NR

\section{Risk of bias}

\begin{tabular}{|c|c|c|}
\hline Bias & Authors' judgement & Support for judgement \\
\hline $\begin{array}{l}\text { Adequate sequence gener- } \\
\text { ation? }\end{array}$ & Low risk & $\begin{array}{l}\text { Quote: "Patients were randomized using sealed envelopes containing group } \\
\text { assignments and a sequence generated using a table of random numbers." p } \\
203\end{array}$ \\
\hline $\begin{array}{l}\text { Adequate allocation con- } \\
\text { cealement? }\end{array}$ & Low risk & $\begin{array}{l}\text { Quote: "Patients, their clinicians, and research staff were not aware of pa- } \\
\text { tients' group assignment until after they consented to participate and the en- } \\
\text { velope was opened." p } 203\end{array}$ \\
\hline $\begin{array}{l}\text { Similar baseline outcome } \\
\text { measurements? }\end{array}$ & High risk & $\begin{array}{l}\text { Judgement comment: large baseline imbalance in the use of ophthalmology } \\
\text { services (intervention 69\%, comparator 41\%). See Table } 2 \text { p } 205\end{array}$ \\
\hline $\begin{array}{l}\text { Similar baseline character- } \\
\text { istics? }\end{array}$ & Low risk & $\begin{array}{l}\text { Quote: "Intervention and control groups had similar characteristics at base- } \\
\text { line." p } 204\end{array}$ \\
\hline $\begin{array}{l}\text { Incomplete outcome data } \\
\text { addressed? }\end{array}$ & Low risk & $\begin{array}{l}\text { Judgement comment: approx. } 90 \% \text { follow-up and missing data balanced } \\
\text { across study arms }\end{array}$ \\
\hline $\begin{array}{l}\text { Knowledge of allocated in- } \\
\text { tervention prevented? }\end{array}$ & Low risk & $\begin{array}{l}\text { Quote: "Data on patients' use of specialty outpatient services were obtained } \\
\text { from electronic utilization databases and survey self-reports." p } 204\end{array}$ \\
\hline & & $\begin{array}{l}\text { Judgement comment: although blinding of outcome assessor not reported, } \\
\text { unlikely to influence outcome }\end{array}$ \\
\hline $\begin{array}{l}\text { Protected against contam- } \\
\text { ination? }\end{array}$ & Low risk & Judgement comment: control group unlikely to have received the intervention \\
\hline $\begin{array}{l}\text { Free from selective out- } \\
\text { come reporting? }\end{array}$ & Unclear risk & $\begin{array}{l}\text { Judgement comment: no protocol or trial registry entry available and there- } \\
\text { fore not possible to assess }\end{array}$ \\
\hline Other risks of bias? & Low risk & Judgement comment: no evidence of other sources of bias \\
\hline
\end{tabular}


Study aim: to investigates the outcomes and costs of an educational and telephone intervention on dilated fundus examination follow-up adherence in patients with diabetes

Study design: parallel-group RCT

\section{Participants}

Country: USA

Setting: tertiary eye-care centre

Total number of participants: 356

Percentage male: $42 \%$

Diabetes type: NR

Average age (SD): $60.7 \mathrm{yrs}(12.6)$

Inclusion criteria: adults ( $\geq 18$ years old) with diabetes who had been previously evaluated in the eye clinic, and had been recommended for a follow-up dilated fundus examination

\section{Exclusion criteria: NR}

Interventions

Intervention arm 1 (mailed intervention) $(\mathbf{n}=\mathbf{1 1 7})$ : personalised letter encouraging scheduling a dilated fundus examination and a brochure about diabetic eye disease and reminder card and automatic reminder call the day before the scheduled appointment

Intervention arm 2 (telephone intervention) ( $\mathbf{n}=\mathbf{1 2 0}$ ): standard reminder letter 1 month prior to exam due date followed by a personal telephone call offering assistance in scheduling an appointment and a reminder letter 3 weeks prior to appointment and automatic reminder call the day before the scheduled appointment

Comparator $(\mathbf{n}=\mathbf{1 1 9})$ : usual care (standard reminder letter 1 month prior to exam due date and automatic reminder call the day before the scheduled appointment)

Duration: 3 months

Outcomes

Primary outcome: obtaining a dilated fundus examination within 90 days of the recommended follow-up date

Secondary outcomes: costs of delivering the intervention

Baseline screening attendance (control group): NR

Notes

Date conducted: November 2012 to February 2013

Trial registration number: NR

Sources of funding: US Centers for Disease Control and Prevention

Declaration of interest: NR

\begin{tabular}{lll}
\hline Risk of bias & Authors' judgement & Support for judgement \\
\hline Bias & Low risk & $\begin{array}{l}\text { Quote: "...randomized within age strata ( }<65 \text { and }>65 \text {-years) using the method } \\
\text { of random permuted block" } \mathrm{p} 254\end{array}$ \\
\hline $\begin{array}{l}\text { Adequate sequence gener- } \\
\text { ation? }\end{array}$ & $\begin{array}{l}\text { Quote: "The study personnel in charge of randomization did not participate in } \\
\text { the interventions." } \mathrm{p} 254\end{array}$ \\
\hline $\begin{array}{l}\text { Adequate allocation con- } \\
\text { cealement? }\end{array}$ & Low risk & \\
\hline
\end{tabular}


Pizzi 2015 (Continued)

\begin{tabular}{lll}
$\begin{array}{l}\text { Similar baseline outcome } \\
\text { measurements? }\end{array}$ & Unclear risk & Not reported \\
\hline $\begin{array}{l}\text { Similar baseline character- } \\
\text { istics? }\end{array}$ & Low risk & $\begin{array}{l}\text { Quote: "There were no statistically significant differences in demographics } \\
\text { among the three study groups (Table 1)" p 257 }\end{array}$ \\
\hline $\begin{array}{l}\text { Incomplete outcome data } \\
\text { addressed? }\end{array}$ & Low risk & Judgement comment: all outcome data reported (see Table 2 p 258) \\
\hline $\begin{array}{l}\text { Knowledge of allocated in- } \\
\text { tervention prevented? }\end{array}$ & Unclear risk & Not reported \\
\hline $\begin{array}{l}\text { Protected against contam- } \\
\text { ination? }\end{array}$ & Low risk & $\begin{array}{l}\text { Judgement comment: it is unlikely that the control group received the active } \\
\text { interventions }\end{array}$ \\
\hline $\begin{array}{l}\text { Free from selective out- } \\
\text { come reporting? }\end{array}$ & Unclear risk & $\begin{array}{l}\text { Judgement comment: no protocol or trial registry entry available and there- } \\
\text { fore not possible to assess }\end{array}$ \\
\hline \begin{tabular}{l} 
Other risks of bias? \\
\hline
\end{tabular} & Low risk & \begin{tabular}{l} 
Judgement comment: no evidence of other risks of bias \\
\hline
\end{tabular}
\end{tabular}

Prela 2000

Methods Study aim: to evaluate the use of a single direct mailed reminder on rate of annual eye examinations in people with diabetes

Study design: parallel-group RCT

\begin{tabular}{ll}
\hline Participants & Country: USA \\
Setting: Medicare beneficiaries & Total number of participants: 6546 \\
& Percentage male: NR \\
& Diabetes type: NR \\
& Average age (SD): NR \\
& Inclusion criteria: Medicare beneficiaries with diabetes (defined by International Classification of Dis- \\
& eases 9th revision. Clinical Modification ICD-9-CM codes of $250 . X X)$ \\
& Exclusion criteria: NR \\
Intervention ( $\mathbf{n}=\mathbf{4 0 9 2}$ ): mailed intervention reinforcing the importance of annual eye examinations \\
Comparator ( $\mathbf{n}=\mathbf{2 4 5 4 ) : ~ u s u a l ~ c a r e ~ ( n o t ~ s p e c i f i e d ) ~}$ \\
Duration: 6 months
\end{tabular}

Outcomes Primary outcome: claims for eye examinations; defined by Physicians Current Procedural Terminology, 4th Edition (CPT-4) codes 99201 - 99205

Secondary outcomes: none

Baseline screening attendance (control group): $48.4 \%$

Notes Date conducted: 1994 to 1995


Prela 2000 (Continued)

\section{Trial registration number: NR}

Sources of funding: US Centers for Disease Control and Prevention

Declaration of interest: NR

\section{Risk of bias}

\begin{tabular}{|c|c|c|}
\hline Bias & Authors' judgement & Support for judgement \\
\hline $\begin{array}{l}\text { Adequate sequence gener- } \\
\text { ation? }\end{array}$ & Unclear risk & Not reported \\
\hline $\begin{array}{l}\text { Adequate allocation con- } \\
\text { cealement? }\end{array}$ & Unclear risk & Not reported \\
\hline $\begin{array}{l}\text { Similar baseline outcome } \\
\text { measurements? }\end{array}$ & Low risk & $\begin{array}{l}\text { Judgement comment: baseline retinal exams reported and balanced across } \\
\text { study arms (see Table } 2 \text { p259) }\end{array}$ \\
\hline $\begin{array}{l}\text { Similar baseline character- } \\
\text { istics? }\end{array}$ & Low risk & $\begin{array}{l}\text { Quote: "The groups were comparable with regard to age, gender and use of } \\
\text { preventative health services" p } 259 \text { (see Table 2) }\end{array}$ \\
\hline $\begin{array}{l}\text { Incomplete outcome data } \\
\text { addressed? }\end{array}$ & Low risk & $\begin{array}{l}\text { Judgement comment: low attrition, outcome data reported on }>90 \% \text { (see Ta- } \\
\text { ble } 4 \text { p 260) }\end{array}$ \\
\hline $\begin{array}{l}\text { Knowledge of allocated in- } \\
\text { tervention prevented? }\end{array}$ & Low risk & $\begin{array}{l}\text { Judgement comment: outcome data were obtained from Medicare claims } \\
\text { databases }\end{array}$ \\
\hline $\begin{array}{l}\text { Protected against contam- } \\
\text { ination? }\end{array}$ & Low risk & $\begin{array}{l}\text { Judgement comment: it is unlikely that the control group received the mailed } \\
\text { intervention }\end{array}$ \\
\hline $\begin{array}{l}\text { Free from selective out- } \\
\text { come reporting? }\end{array}$ & Unclear risk & $\begin{array}{l}\text { Judgement comment: no protocol or trial registry entry available and there- } \\
\text { fore not possible to assess }\end{array}$ \\
\hline Other risks of bias? & Low risk & Judgement comment: no evidence of other risks of bias \\
\hline
\end{tabular}

Prezio 2014

Methods

Methods

\begin{abstract}
Study aim: to determine the impact of a culturally-tailored diabetes education programme led by a community health worker (CHW) on the HbAlc, blood pressure, BMI and lipid status of uninsured Mexican Americans with diabetes
\end{abstract}

Study design: parallel-group RCT

\section{Country: USA}

Setting: primary care (faith-based urban health services clinic serving exclusively uninsured patients of largely Mexican American origin)

\section{Total number of participants: 180}

Percentage male: $39.5 \%$

Diabetes type: type 2

Average age (SD): $46.8 \mathrm{yrs}(10.9)$ 
Inclusion criteria: eligible patients were uninsured, had no previous exposure to the Community Diabetes Education (CODE) programme, were 18 to 75 years of age, had type 2 diabetes either treated with anti-diabetic medications or diet-controlled.

Exclusion criteria: advanced complications from diabetes; pregnancy

\begin{abstract}
Interventions
Intervention ( $\mathbf{n}=\mathbf{9 0}$ ): community diabetes educational programme delivered by CHW. 3 educational modules were delivered during individual 1-hour sessions over the first 8 weeks. These sessions covered areas recommended by the ADA. The CHW facilitated immediate physician contact to address acute problems, assisted with pharmacy refills, and arranged specialty visits such as dental care and dilated retinal exams. Participants were provided with a blood glucose monitor and testing strips free of charge and instructed in correct use of the device by medical assistants
\end{abstract}

Comparator $(\mathbf{n}=\mathbf{9 0})$ : usual medical care at the discretion of the clinic physicians. Participants in this group were provided with a blood glucose monitor and testing strips free of charge and instructed in correct use of the device by medical assistants. Culturally-tailored printed diabetes education materials were provided by physicians and clinic staff

Duration: 6 months

Outcomes Primary outcome: impact of the intervention on HbA1c, lipid status, blood pressure and BMI

Secondary outcomes: participants' attitudes and knowledge about diabetes self-management, ADA standards of care (including annual dilated fundus examination)

Baseline screening attendance (control group): $6.7 \%$

Dotes conducted: 2006
Trial registration number: NCT00151190
Sources of funding: University of Texas School of Public Health, Institute for Faith-Health Research,
Dallas

Declaration of interest: none declared

Study protocol has been published: www.ncbi.nlm.nih.gov/pubmed/17431443

\title{
Risk of bias
}

Bias Authors' judgement Support for judgement

Adequate sequence gener- Low risk ation?
Quote: "All patients were given informed consent in the preferred language of the study subject followed by (1:1) assignment to either the intervention or control groups using a computer generated randomization schedule." see Prezio 2013 p 20

Adequate allocation con- Unclear risk Not reported
cealement?

Similar baseline outcome Low risk measurements?

Judgement comment: baseline retinal exams reported and similar across study arms (see Table 3 p 129)

Similar baseline character- Low risk istics?
Quote: "No significant differences in baseline clinical, demographic, and behavioral characteristics were found between the intervention and control groups, with the exception that significantly more control group participants were employed at study entry $(P=.02$; Table 2)." Table 2 p 127

Judgement comment: employment status may have influenced attendance for retinopathy screening 
Prezio 2014 (Continued)

\begin{tabular}{|c|c|c|}
\hline $\begin{array}{l}\text { ncomplete outcome data } \\
\text { ddressed? }\end{array}$ & Low risk & $\begin{array}{l}\text { Judgement comment: intention-to-treat analysis. All participants accounted } \\
\text { for. See CONSORT flow diagram p } 21 \text { Prezio } 2013\end{array}$ \\
\hline
\end{tabular}
addressed? for. See CONSORT flow diagram p 21 Prezio 2013

Knowledge of allocated in- Unclear risk

Not reported

tervention prevented?

Protected against contam- High risk ination?

Judgement comment: all participants were from the same faith-based community services clinic and no evidence that the study was protected from contamination

$\begin{array}{ll}\begin{array}{l}\text { Free from selective out- } \\ \text { come reporting? }\end{array} & \text { Low risk } \\ \end{array}$

Other risks of bias? Low risk Judgment comment: no evidence of other risks of bias

Rosenkranz 1996

$\begin{array}{ll}\text { Methods } & \text { Study aim: to study whether polaroid fundus photography during a patient consultation would influ- } \\ \text { ence future screening behaviour for diabetic retinopathy }\end{array}$

Study design: parallel-group RCT

Participants

Country: Germany

Setting: Diabetes clinic within the University of Düsseldorf

Total number of participants: 103

Percentage male: $61.1 \%$

Diabetes type: type 1 and 2 (87\% type 2)

Average age (SD): NR

Inclusion criteria: patients with diabetes living within a $100 \mathrm{Km}$ radius of the clinic

Exclusion criteria: diabetic retinopathy or treatment for diabetic retinopathy; patients with glaucoma or cataract

Interventions

Intervention arm $\mathbf{1}(\mathbf{n}=\mathbf{3 5})$ : Group B. Polaroid photograph taken, shown and explained to the participant. The photograph was then given to the participant to take home. Results of all clinical investigations explained to participant and also included in a subsequent letter which contained a recommendation for an eye exam performed by an ophthalmologist and the time frame for this exam.

Intervention arm $2(\mathbf{n}=\mathbf{3 1})$ : Group C. Polaroid photograph taken, shown and explained to the participant. The photograph was then retained in the participant's file. Results of all clinical investigations explained to participant and also included in a subsequent letter which contained a recommendation for an eye exam performed by an ophthalmologist and the time frame for this exam.

Comparator ( $\mathbf{n}=\mathbf{3 7}$ ): Group A. Polaroid photograph of fundus taken but not shown to participant. Results of all clinical investigations explained to participant and also included in a subsequent letter which contained a recommendation for an eye exam performed by an ophthalmologist and the time frame for this exam

Duration: 12 months

Outcomes

Primary outcome: attendance for diabetic retinopathy screening

Secondary outcomes: factors affecting screening attendance 
Rosenkranz 1996 (Continued)

Baseline screening attendance (control group): NR

Notes

Date conducted: NR

Trial registration number: NR

Sources of funding: NR

Declaration of interest: NR

\begin{tabular}{|c|c|c|}
\hline \multicolumn{3}{|l|}{ Risk of bias } \\
\hline Bias & Authors' judgement & Support for judgement \\
\hline $\begin{array}{l}\text { Adequate sequence gener- } \\
\text { ation? }\end{array}$ & Unclear risk & Not reported \\
\hline $\begin{array}{l}\text { Adequate allocation con- } \\
\text { cealement? }\end{array}$ & Unclear risk & Not reported \\
\hline $\begin{array}{l}\text { Similar baseline outcome } \\
\text { measurements? }\end{array}$ & Unclear risk & Not reported \\
\hline $\begin{array}{l}\text { Similar baseline character- } \\
\text { istics? }\end{array}$ & Low risk & $\begin{array}{l}\text { Judgement comment: similar demographic characteristics across the } 3 \text { arms } \\
\text { of the study for age, gender and socioeconomic status (see Table } 1 \text { p 70) }\end{array}$ \\
\hline $\begin{array}{l}\text { Incomplete outcome data } \\
\text { addressed? }\end{array}$ & Low risk & $\begin{array}{l}\text { Judgement comment: all participants were followed up and reported (see Ta- } \\
\text { ble } 2 \text { p 71) }\end{array}$ \\
\hline $\begin{array}{l}\text { Knowledge of allocated in- } \\
\text { tervention prevented? }\end{array}$ & Unclear risk & Not reported \\
\hline $\begin{array}{l}\text { Protected against contam- } \\
\text { ination? }\end{array}$ & High risk & $\begin{array}{l}\text { Judgement comment: given the nature of the intervention it is possible that } \\
\text { the control group received the intervention }\end{array}$ \\
\hline $\begin{array}{l}\text { Free from selective out- } \\
\text { come reporting? }\end{array}$ & Unclear risk & $\begin{array}{l}\text { Judgement comment: no protocol or trial registry entry available and there- } \\
\text { fore not possible to assess }\end{array}$ \\
\hline Other risks of bias? & High risk & $\begin{array}{l}\text { Judgement comment: patients with existing diabetic retinopathy or previously } \\
\text { treated for diabetic retinopathy were excluded }\end{array}$ \\
\hline
\end{tabular}

Schnipper 2010

Methods Study aim: to evaluate whether a new document-based clinical decision-support system is effective in improving the quality of care in coronary artery disease and diabetes

Study design: cluster-RCT

Participants Country: USA

Setting: Primary care practices at Brigham and Women's Hospital and Massachusetts General Hospital

Number of clusters: 10

Number of providers: 239

Total number of patients: 7009 ( $71.5 \%$ with diabetes) 
Schnipper 2010 (Continued)

\section{Percentage male: NR}

Diabetes type: type 1 and 2

Average age (SD): NR

Inclusion criteria: patients with type 1 or type 2 diabetes

Exclusion criteria: patients already under the regular care of an ophthalmologist

Interventions

Intervention ( $\mathbf{5}$ clusters, $\mathbf{n}=\mathbf{3 4 3 1}$ ): 'smart form' with reminders. Document-based clinical support system built into an electronic heath record. The system highlights missing and 'requests' missing data

Comparator ( $\mathbf{5}$ clusters, $\mathbf{n}=\mathbf{3 5 7 8})$ : usual care (not specified)

Duration: 9 months

Outcomes

Primary outcome: mean \% of deficiencies in disease management within 1 month of a clinic visit (including eye examination documentation-diabetes patients only)

Secondary outcomes: NR

Baseline screening attendance (control group): NR

Notes

Date conducted: 2008

Trial registration number: NR

Sources of funding: Agency for Healthcare and Quality

Declaration of interest: none declared

\section{Risk of bias}

\begin{tabular}{|c|c|c|}
\hline Bias & Authors' judgement & Support for judgement \\
\hline $\begin{array}{l}\text { Adequate sequence gener- } \\
\text { ation? }\end{array}$ & Low risk & $\begin{array}{l}\text { Quote: "Primary care physicians were assigned to receive the Smart Form or } \\
\text { usual care on the basis of random number generation in Microsoft Excel (Red- } \\
\text { mond, WA)." } \\
\text { p SP73 }\end{array}$ \\
\hline $\begin{array}{l}\text { Adequate allocation con- } \\
\text { cealement? }\end{array}$ & Low risk & $\begin{array}{l}\text { Judgement comment: unit of allocation at the level of the primary care prac- } \\
\text { tice and allocation performed prior to the start of the study }\end{array}$ \\
\hline $\begin{array}{l}\text { Similar baseline outcome } \\
\text { measurements? }\end{array}$ & Unclear risk & Not reported \\
\hline $\begin{array}{l}\text { Similar baseline character- } \\
\text { istics? }\end{array}$ & High risk & $\begin{array}{l}\text { Judgement comment: a number of baseline differences in characteristics in- } \\
\text { cluding: female }(P<0.001) \text {, number of problems on problem list }(P<0.001) \text {, } \\
\text { race }(P<0.001) \text {, primary insurance }(P=0.002) \text {, median household income }(P= \\
0.01)\end{array}$ \\
\hline $\begin{array}{l}\text { Incomplete outcome data } \\
\text { addressed? }\end{array}$ & Unclear risk & Not reported \\
\hline $\begin{array}{l}\text { Knowledge of allocated in- } \\
\text { tervention prevented? }\end{array}$ & Unclear risk & Not reported \\
\hline $\begin{array}{l}\text { Protected against contam- } \\
\text { ination? }\end{array}$ & Low risk & $\begin{array}{l}\text { Judgement comment: allocation by primary care practice; it is unlikely that } \\
\text { the control group received the intervention }\end{array}$ \\
\hline
\end{tabular}


Schnipper 2010 (Continued)

Free from selective out- Unclear risk Judgement comment: no protocol or trial registry entry available and therecome reporting? fore not possible to assess

Other risks of bias?

Simon 2010

Methods Study aim: to assess the effects of automated telephone outreach with speech recognition on diabetes-related testing.

Study design: parallel-group RCT

$\begin{array}{ll}\text { Participants } & \text { Country: USA } \\ & \text { Setting: Harvard Pilgrim Healthcare Institute }\end{array}$

Total number of participants: 1200

Percentage male: $61.6 \%$

Diabetes type: $95 \%$ type 2

Average age (SD): 51.1 yrs (10.9)

Inclusion criteria: adult health plan members with diabetes overdue for routine testing (sample limited to individuals with no insurance claim for a dilated eye examination in the prior year and no claim for 1 or more of the following tests: HbA1c, LDL cholesterol, or microalbumin)

\section{Exclusion criteria: NR}

Interventions

Intervention ( $\mathbf{n = 6 0 0 ) : ~ a ~ c o m p u t e r i s e d ~ t e l e p h o n e ~ s y s t e m ~ p l a c e d ~} 3$ calls to the participant's home, encouraging the participant to fulfil recommended testing. The automated system offered a live telephone call back to assist in scheduling tests and also offered to send participants the following items: 1) a voucher that would allow the provider to waive the co-payment for a dilated eye examination; 2) an educational nutrition video; 3) a cookbook; or 4) a pill box.

Comparator $(\mathbf{n}=\mathbf{6 0 0})$ : usual care (not specified)

Duration: 12 months

Outcomes

Primary outcome: attendance for a dilated fundus examination

Secondary outcomes: tests for glycaemia, hyperlipidaemia, and nephropathy

Baseline screening attendance (control group): $0 \%$

Notes Date conducted: 2006

Trial registration number: NCT00790530

Sources of funding: ADA, Harvard Pilgrim Health Care Institute

Declaration of interest: none declared

Outcome data obtained from Supplementary Figure 2 (online supplementary appendix)

\section{Risk of bias}

\section{Bias}

Authors' judgement Support for judgement 
Simon 2010 (Continued)
Adequate sequence gener-
Unclear risk
Not reported ation?

Adequate allocation con- Unclear risk $\quad$ Not reported
cealement?

\begin{tabular}{|c|c|c|}
\hline $\begin{array}{l}\text { Similar baseline outcome } \\
\text { measurements? }\end{array}$ & Unclear risk & Not reported \\
\hline $\begin{array}{l}\text { Similar baseline character- } \\
\text { istics? }\end{array}$ & Low risk & $\begin{array}{l}\text { Quote: "Compared with the usual care group, the intervention group was } \\
\text { younger ( } 50 \text { vs. } 52 \text { years, } P=0.02 \text { ) and had a greater proportion of men ( } 64 \\
\text { vs. } 41 \%, P=0.04) \text {; the groups were comparable on other socio-demographic } \\
\text { measures and clinical indicators as shown in supplementary Table 2." p } 1453 \\
\text { Judgement comment: baseline differences unlikely to influence outcome }\end{array}$ \\
\hline $\begin{array}{l}\text { Incomplete outcome data } \\
\text { addressed? }\end{array}$ & Low risk & Judgement comment: no missing data \\
\hline $\begin{array}{l}\text { Knowledge of allocated in- } \\
\text { tervention prevented? }\end{array}$ & Low risk & $\begin{array}{l}\text { Judgement comment: outcomes were obtained from automated clinical ad- } \\
\text { ministrative databases }\end{array}$ \\
\hline $\begin{array}{l}\text { Protected against contam- } \\
\text { ination? }\end{array}$ & Low risk & $\begin{array}{l}\text { Judgement comment: it is unlikely that the control group received telephone } \\
\text { intervention }\end{array}$ \\
\hline $\begin{array}{l}\text { Free from selective out- } \\
\text { come reporting? }\end{array}$ & Unclear risk & $\begin{array}{l}\text { Judgement comment: trial retrospectively registered and not possible to as- } \\
\text { sess }\end{array}$ \\
\hline Other risks of bias? & Low risk & Judgement comment: no evidence of other risks of bias \\
\hline
\end{tabular}

\title{
Simpson 2011
}

Methods $\begin{aligned} & \text { Study aim: to evaluate the effect of adding pharmacists to the primary care team on the management } \\ & \text { of patients with type } 2 \text { diabetes }\end{aligned}$

Study design: parallel-group RCT

Participants

\author{
Country: Canada \\ Setting: 2 public family medicine clinics (primary care)
}

Total number of patients: 260

Percentage male: $42.7 \%$

Diabetes type: type 2

Average age (SD): 59.1 yrs (11.6)

Inclusion criteria: patients were eligible if they had type 2 diabetes, were regularly seen by the primary care team, and did not qualify for urgent specialist referral and assessment

Exclusion criteria: patients who were followed in specialty clinics for diabetes, hypertension, or dyslipidaemia; who were cognitively impaired; who were not responsible for their own medication administration; or who were unable to communicate in English 
Simpson 2011 (Continued)

Interventions
Intervention ( $\mathbf{n}=\mathbf{1 3 1}$ ): pharmacists performed medication assessments and limited history and physical examinations and provided guideline-concordant recommendations to optimise medication management.

Comparator $(\mathbf{n}=\mathbf{1 2 9})$ : usual care (not specified)

Duration: 12 months

Primary outcome: achievement of a clinically-important reduction in blood pressure, defined as a $10 \%$ decrease in systolic blood pressure at 1 year

Secondary outcomes: absolute change in SBP from baseline to 1 year, achievement of recommended blood pressure targets $(<130 / 80 \mathrm{mmHg}$ ), and antihypertensive medication changes. Healthcare-related contacts during the study period (including visits to an ophthalmologist or optometrist)

Baseline screening attendance (control group): NR

Notes Date conducted: 2009

Trial registration number: ISRCTN97121854

Sources of funding: Canadian Diabetes Association, the Institute of Health Economics, and the Alberta Heritage Foundation for Medical Research

Declaration of interest: none declared

\section{Risk of bias}

\begin{tabular}{|c|c|c|}
\hline Bias & Authors' judgement & Support for judgement \\
\hline $\begin{array}{l}\text { Adequate sequence gener- } \\
\text { ation? }\end{array}$ & Low risk & $\begin{array}{l}\text { Quote: "A central randomization service (www.epicore.ualberta.ca) provided } \\
\text { computer generated random sequences stratified by the primary care clinic } \\
\text { for treatment allocation." p } 21\end{array}$ \\
\hline $\begin{array}{l}\text { Adequate allocation con- } \\
\text { cealement? }\end{array}$ & Low risk & $\begin{array}{l}\text { Quote: "Pharmacists, analysts, and investigators were unaware of the block } \\
\text { size and allocation sequence to preserve allocation concealment." p } 21\end{array}$ \\
\hline $\begin{array}{l}\text { Similar baseline outcome } \\
\text { measurements? }\end{array}$ & Unclear risk & Not reported \\
\hline $\begin{array}{l}\text { Similar baseline character- } \\
\text { istics? }\end{array}$ & Low risk & $\begin{array}{l}\text { Quote: "Baseline characteristics were well balanced between the groups (Ta- } \\
\text { ble 1)." p } 23\end{array}$ \\
\hline \multirow[t]{2}{*}{$\begin{array}{l}\text { Incomplete outcome data } \\
\text { addressed? }\end{array}$} & Low risk & $\begin{array}{l}\text { Quote: "There were no differences in age, sex, diabetes duration, or baseline } \\
\text { blood pressure between the patients who did or did not complete the study." } p \\
22\end{array}$ \\
\hline & & $\begin{array}{l}\text { Judgement comment: intention-to-treat analysis analysis and reasons for loss- } \\
\text { es to follow-up provided and balanced across study arms }\end{array}$ \\
\hline $\begin{array}{l}\text { Knowledge of allocated in- } \\
\text { tervention prevented? }\end{array}$ & Unclear risk & $\begin{array}{l}\text { Judgement comment: not clear whether eye-screening outcome assessors } \\
\text { were masked }\end{array}$ \\
\hline \multirow[t]{2}{*}{$\begin{array}{l}\text { Protected against contam- } \\
\text { ination? }\end{array}$} & High risk & $\begin{array}{l}\text { Quote : ".. there was the possibility of "contamination" or "cointervention" be- } \\
\text { cause both intervention and control patients were drawn from the same pri- } \\
\text { mary care team." }\end{array}$ \\
\hline & & p 25 \\
\hline
\end{tabular}


Simpson 2011 (Continued)

Free from selective out- Low risk_ Judgement comment: reported outcomes consistent with trial registry come reporting? ISRCTN97121854

Other risks of bias?

Low risk

Judgement comment: no evidence of other sources of bias

Sonnichsen 2010

\begin{tabular}{|c|c|}
\hline Methods & $\begin{array}{l}\text { Study aim: to evaluate whether a disease management programme consisting of physician and pa- } \\
\text { tient education, standardised documentation and therapeutic goals improves metabolic control } \\
\text { (HbA1c) and quality of care for adults with type } 2 \text { diabetes managed in primary care } \\
\text { Study design: cluster-RCT }\end{array}$ \\
\hline Participants & $\begin{array}{l}\text { Country: Austria } \\
\text { Setting: primary care practices with a contract with the public health insurance in Austria (province of } \\
\text { Salzburg) } \\
\text { Number of clusters: } 6 \\
\text { Number of providers: } 92 \\
\text { Total number of patients: } 1494 \\
\text { Percentage male: } 52.2 \% \\
\text { Diabetes type: type } 2 \\
\text { Average age (SD): } 65.5 \text { yrs (10.4) } \\
\text { Inclusion criteria: all patients with type } 2 \text { diabetes willing to participate in the study } \\
\text { Exclusion criteria: dementia/psychiatric illness with inability to participate or to give informed con- } \\
\text { sent }\end{array}$ \\
\hline Interventions & $\begin{array}{l}\text { Intervention ( } \mathbf{3} \text { clusters, } \mathbf{n}=\mathbf{6 5 4} \text { ): Disease Management Programme (DMP) containing the following } \\
\text { modules: } \\
\text { - standardised documentation of physical examination, laboratory findings, and diabetes complica- } \\
\text { tions in a DMP-form once a year } \\
\text { - structured interdisciplinary care according to the guidelines of the Austrian Diabetes Association } \\
\text { - agreement on therapeutic goals in a shared patient-physician decision-making process at 3-monthly } \\
\text { intervals } \\
\text { Comparator ( } \mathbf{3} \text { clusters, } \mathbf{n}=\mathbf{8 4 0} \text { ): usual care (not specified) } \\
\text { Duration: } 12 \text { months }\end{array}$ \\
\hline
\end{tabular}

Outcomes

Primary outcome: change in HbA1c from baseline to 12 months

Secondary outcomes: improvement in systolic or diastolic blood pressure, lipids, and BMI; measures of process quality including the frequency of $\mathrm{HbAlc}$ measurements, eye and foot examinations; participation in patient education

Baseline screening attendance (control group): NR 
Sonnichsen 2010 (Continued)

Sources of funding: Paracelsus Medical University, Public Health Insurance of Salzburg, Salzburg Sav-

ings Bank, Roche Diagnostics

Declaration of interest: none declared

\section{Risk of bias}

\begin{tabular}{lll}
\hline Bias & Authors' judgement & Support for judgement \\
\hline $\begin{array}{l}\text { Adequate sequence gener- } \\
\text { ation? }\end{array}$ & Low risk & $\begin{array}{l}\text { Quote: "...cluster-randomisation at the level of the districts was performed } \\
\text { with computerised sequence generation." } \mathrm{p} 4\end{array}$ \\
\hline $\begin{array}{l}\text { Adequate allocation con- } \\
\text { cealement? }\end{array}$ & Low risk & $\begin{array}{l}\text { Quote: "To assure concealment of allocation at the physician level, GPs and in- } \\
\text { ternists were not told whether they would be in the intervention or the control } \\
\text { group until after obtaining their consent to participate." p } 4\end{array}$
\end{tabular}

\begin{tabular}{|c|c|c|}
\hline $\begin{array}{l}\text { Similar baseline outcome } \\
\text { measurements? }\end{array}$ & Unclear risk & Not reported \\
\hline
\end{tabular}

Similar baseline character- Low risk istics?
Quote: "Baseline data are shown in table 2. There were no significant differences between the intervention and the control group except for BMI and cholesterol, with intervention patients being slightly heavier and having higher cholesterol levels than controls." p 4

Judgement comment: baseline differences unlikely to influence outcome

Incomplete outcome data High risk

addressed?

Judgement comment: intention-to-treat (ITT) and per-protocol analysis. For ITT, after randomisation, 6 GP practices withdrew before recruiting participants, and 5 in intervention group were excluded since they withdrew consent and did not provide baseline values. The trialists excluded these values and considered it an ITT

\section{Knowledge of allocated in- High risk} tervention prevented?

Quote: "As typical for pragmatic trials, blinding was not possible and the knowledge of being in the intervention or control group may have influenced the result." p 8

\begin{tabular}{lll}
\hline $\begin{array}{l}\text { Protected against contam- } \\
\text { ination? }\end{array}$ & Low risk & $\begin{array}{l}\text { Judgement comment: allocation by primary care practice and it is unlikely } \\
\text { that the control group received the intervention }\end{array}$ \\
\hline $\begin{array}{l}\text { Free from selective out- } \\
\text { come reporting? }\end{array}$ & Low risk & $\begin{array}{l}\text { Judgement comment: reported outcomes consistent with trial registry } \\
\text { ISCTN27414162 }\end{array}$
\end{tabular}

Other risks of bias? Low risk Judgement comment: no evidence of other sources of bias

Steyn 2013

$\begin{array}{ll}\text { Methods } & \begin{array}{l}\text { Study aim: to evaluate the effect introducing a structured clinical record (with embedded national } \\ \text { guideline recommendations) and training of healthcare providers in its use, on the quality of care for } \\ \text { diabetes and hypertension }\end{array}\end{array}$

Study design: cluster-RCT

Participants Country: South Africa

Setting: public sector primary healthcare clinics (Community Health Centres) in working class residential area in Cape Town

\section{Number of clusters: 18}


Steyn 2013 (Continued)

\section{Number of providers: NR \\ Total number of patients: 456}

Percentage male: $26.1 \%$

Diabetes type: types 1 and 2 (92\% type 2)

Average age (SD): 58.3 yrs (10.9)

Inclusion criteria: $\geq 15$ years; a documented attendance at the particular community health clinic with at least 4 visits during the previous year for hypertension or diabetes; and having received treatment for these conditions at each visit

Exclusion criteria: unable to provide answers to a questionnaire

- structured record, which incorporated the National Guidelines for the management of patients with diabetes or hypertension

- physician educational package consisted of an outreach visit by a recognised local diabetes and hypertension expert

Comparator (9 clusters, $\mathbf{n}=\mathbf{2 1 7}$ participants): usual care (guidelines passively disseminated by the National Department of Health)

Duration: 12 months

Outcomes Primary outcome: mean level of HbAlc

Secondary outcomes: proportion of participants with diabetes BP $<130 / 85 \mathrm{mmHg}$ ); proportion with uncontrolled glycaemia (\% with $\mathrm{HbAlc}>7 \%$ ); proportions of participants with recorded examinations for complications (retinopathy, nephropathy, foot problems)

Baseline screening attendance (control group): $8.8 \%$

Date conducted: 2000
Trial registration number: Pan African Clinical Trial Registry (www.pactr.org) PACTR201303000493351
Sources of funding: South African Medical Research Council; unrestricted grant from Hoechst, Marion,
Roussel
Declaration of interest: 1 author (NL) received honoraria from Novartis and travel support from Novo
Nordisk, Eli Lilly Laboratories and Sanofi Aventis; all other authors reported no conflict of interest

Risk of bias

\begin{tabular}{lll}
\hline Bias & Authors' judgement & Support for judgement \\
\hline $\begin{array}{l}\text { Adequate sequence gener- } \\
\text { ation? }\end{array}$ & Low risk & $\begin{array}{l}\text { Quote: "Study clinics were randomly allocated, by stratum, to intervention or } \\
\text { control using a computer-generated list of random numbers." p } 3\end{array}$ \\
\hline $\begin{array}{l}\text { Adequate allocation con- } \\
\text { cealement? }\end{array}$ & Low risk & $\begin{array}{l}\text { Judgement comment: unit of allocation at the level of the primary care prac- } \\
\text { tice and allocation performed prior to the start of the study }\end{array}$ \\
\hline $\begin{array}{l}\text { Similar baseline outcome } \\
\text { measurements? }\end{array}$ & Low risk & $\begin{array}{l}\text { Judgement comment: similar rates of eye examinations between arms at } \\
\text { baseline (intervention 18\%, control 9\%) }\end{array}$ \\
\hline $\begin{array}{l}\text { Similar baseline character- } \\
\text { istics? }\end{array}$ & Low risk & Judgement comment: similar baseline characteristics (Table 1 p 5) \\
\hline
\end{tabular}


Steyn 2013 (Continued)

Incomplete outcome data Low risk Judgement comment: low attrition and reasons for missing data provided
addressed?

\begin{tabular}{lll}
$\begin{array}{l}\text { Knowledge of allocated in- } \\
\text { tervention prevented? }\end{array}$ & Unclear risk & Not reported \\
\hline $\begin{array}{l}\text { Protected against contam- } \\
\text { ination? }\end{array}$ & Low risk & $\begin{array}{l}\text { Judgement comment: allocation by primary care practice and it is unlikely } \\
\text { that the control group received the intervention }\end{array}$ \\
\hline $\begin{array}{l}\text { Free from selective out- } \\
\text { come reporting? }\end{array}$ & Unclear risk & $\begin{array}{l}\text { Judgement comment: trial retrospectively registered and therefore not possi- } \\
\text { ble to assess }\end{array}$ \\
\hline \begin{tabular}{lll} 
Other risks of bias? & Low risk & Judgement comment: no evidence of other sources of bias \\
\hline
\end{tabular}
\end{tabular}

Taylor 2003

Methods Study aim: to evaluate the efficacy of a nurse-care management system designed to improve outcomes in patients with complicated diabetes

Study design: parallel-group RCT

Participants

\section{Country: USA}

Setting: a medical centre in Santa Clara, California

Total number of participants: 169

Percentage male: $53 \%$

Diabetes type: type 1 and type 2

Average age (SD): 55.1 yrs (10.2)

Inclusion criteria: patients with an $\mathrm{HbA1C}>10.0 \%$ and an ICD-9-based diagnosis of diabetes and hypertension, dyslipidaemia, or CVD

Exclusion criteria: did not speak English; not willing or able to participate in the group sessions once a week for 4 weeks; had congestive heart failure as their primary diagnosis; were < 18 years of age; were pregnant; were enrolled in a diabetes management clinic; or fell into the "other" category (e.g. living too far away/moving, deceased, or no-show to baseline appointment)

Interventions

Intervention ( $\mathbf{n}=\mathbf{8 4}$ ): participants met with a nurse-care manager to establish individual outcome goals, attended group sessions once a week for up to 4 weeks, and received telephone calls to manage medications and self-care activities

Comparator $(\mathbf{n}=\mathbf{8 5})$ : usual care (under the treatment of their primary care physician. Each participant received a folder containing diabetes pamphlets and sheet of instructions encouraging them to maintain contact with their personal physician and to attend general diabetes education classes at their medical centre)

Duration: 12 months

Outcomes

Primary outcome: $\%$ of participants meeting process outcome goals at 12 months (including self-reported dilated eye exam); number of physician visits during the study period

Secondary outcomes: participant and physician views regarding the intervention

Baseline screening attendance (control group): $71.2 \%$ 
Taylor 2003 (Continued)

Notes

Date conducted: 2000 to 2001

Trial registration number: NR

Sources of funding: Robert Wood Johnson Foundation

Declaration of interest: NR

\begin{tabular}{|c|c|c|}
\hline \multicolumn{3}{|l|}{ Risk of bias } \\
\hline Bias & Authors' judgement & Support for judgement \\
\hline $\begin{array}{l}\text { Adequate sequence gener- } \\
\text { ation? }\end{array}$ & Unclear risk & Not reported \\
\hline $\begin{array}{l}\text { Adequate allocation con- } \\
\text { cealement? }\end{array}$ & Unclear risk & Note reported \\
\hline $\begin{array}{l}\text { Similar baseline outcome } \\
\text { measurements? }\end{array}$ & Low risk & Judgement comment: similar \% of reported dilated eye exams across arms \\
\hline $\begin{array}{l}\text { Similar baseline character- } \\
\text { istics? }\end{array}$ & Low risk & $\begin{array}{l}\text { Quote: "The demographics of the } 169 \text { patients enrolled in the study can be } \\
\text { seen in Table 1.There were no differences between usual care and intervention } \\
\text { subjects for any of these variables." p } 1060\end{array}$ \\
\hline $\begin{array}{l}\text { Incomplete outcome data } \\
\text { addressed? }\end{array}$ & Unclear risk & $\begin{array}{l}\text { Judgement comment: missing data approx. } 20 \% \text { in intervention group and } \\
17 \% \text { for comparator group (due to dropping out or being lost to follow-up). } \\
\text { Unclear if missing data would influence outcome }\end{array}$ \\
\hline $\begin{array}{l}\text { Knowledge of allocated in- } \\
\text { tervention prevented? }\end{array}$ & Low risk & $\begin{array}{l}\text { Quote: "All eligible patients met with a research assistant blinded to the sub- } \\
\text { ject's random assignment for baseline and follow-up assessments at } 1 \text { year." } p \\
1059\end{array}$ \\
\hline $\begin{array}{l}\text { Protected against contam- } \\
\text { ination? }\end{array}$ & Low risk & Judgement comment: control group unlikely to have received the intervention \\
\hline $\begin{array}{l}\text { Free from selective out- } \\
\text { come reporting? }\end{array}$ & Unclear risk & $\begin{array}{l}\text { Judgement comment: no protocol or trial registry entry available and there- } \\
\text { fore not possible to assess }\end{array}$ \\
\hline Other risks of bias? & Low risk & Judgement comment: no evidence of other sources of bias \\
\hline
\end{tabular}

\title{
Varney 2014
}

Methods

Study aim: to measure the effect of a 6-month telephone coaching intervention on glycaemic control, risk factor status and adherence to diabetes management practices

Study design: parallel-group RCT

Participants

\author{
Country: Australia \\ Setting: hospital diabetes clinic
}

Total number of participants: 94

Percentage male: $68 \%$

Diabetes type: type 2 
Varney 2014 (Continued)

Average age (SD): 61.5 yrs (NR)

Inclusion criteria: adults with type 2 diabetes with $\mathrm{HbA} 1 \mathrm{c}>7 \%$

Exclusion criteria: patients who were unable to provide informed consent, non-English speaking, cognitively impaired, receiving palliative care, severely hearing impaired or without telephone access

Interventions

Intervention ( $\mathbf{n}=\mathbf{4 7}$ ): usual care plus intensive telephone coaching 6 months duration by a dietician experienced in type 2 diabetes management. Participants received an average of 6 sessions

Comparator ( $\mathbf{n}=\mathbf{4 7}$ ): usual care (consisting of attendance at the diabetes clinic 3 - 6 -monthly with GP visits as required)

Duration: 6 months

Primary outcome: HbAlc at 6 months, adjusted for baseline value
Secondary outcomes: adjusted mean HbA1c at 12 months, as well as 6 - and 12 -month adjusted mean
fasting glucose, lipids, BP, weight, waist circumference, BMI, physical activity and Kessler Psychological
Distress Scale score. Participants were asked researcher-generated questions to determine adherence
to guidelines recommending annual foot examinations, biennial eye examinations, annual influenza
vaccinations, pneumococcal vaccination every 5 or 10 years and smoking cessation

Baseline screening attendance (control group): $87.2 \%$

Date conducted: NR
Trial registration number: ACTRN12609000075280 (www.anzctr.org.au)
Sources of funding: St Vincent's Hospital Research Endowment Fund
Declaration of interest: none declared
Additional outcome data obtained from the author

Risk of bias

\begin{tabular}{|c|c|c|}
\hline Bias & Authors' judgement & Support for judgement \\
\hline $\begin{array}{l}\text { Adequate sequence gener- } \\
\text { ation? }\end{array}$ & Low risk & $\begin{array}{l}\text { Quote: "A researcher, not involved in recruitment, randomised participants in- } \\
\text { to intervention and control groups. Computer-generated block randomisation } \\
\text { was undertaken to obtain a one-to-one balanced design." p } 891\end{array}$ \\
\hline $\begin{array}{l}\text { Adequate allocation con- } \\
\text { cealement? }\end{array}$ & Low risk & $\begin{array}{l}\text { Quote: "Allocation blinding was maintained until randomisation, after which } \\
\text { participants and the principal researcher were informed of randomisation out- } \\
\text { come." p } 891\end{array}$ \\
\hline $\begin{array}{l}\text { Similar baseline outcome } \\
\text { measurements? }\end{array}$ & Low risk & $\begin{array}{l}\text { Judgement comment: no differences in baseline eye examinations (see Table } \\
1 \text { p 893) }\end{array}$ \\
\hline $\begin{array}{l}\text { Similar baseline character- } \\
\text { istics? }\end{array}$ & Low risk & $\begin{array}{l}\text { Quote: "Study participants differed from the population attending the dia- } \\
\text { betes clinic in the recruitment period, being younger } 61.4(59.2-63.5) \text { versus } \\
64.1 \text { years }(63.2-65.0, P=0.02) \text {, and being less likely to require an interpreter, } \\
0 \% \text { versus } 29 \%, P<0.001 \text {, reflecting the study's inclusion criteria." p } 892 \\
\text { Judgement comment: baseline difference unlikely to influence outcome }\end{array}$ \\
\hline $\begin{array}{l}\text { Incomplete outcome data } \\
\text { addressed? }\end{array}$ & High risk & $\begin{array}{l}\text { Judgement comment: approximately } 25 \% \text { attrition at } 12 \text { months which may } \\
\text { have biased the results }\end{array}$ \\
\hline
\end{tabular}


Varney 2014 (Continued)

Knowledge of allocated in- Unclear risk Not reported tervention prevented?

\begin{tabular}{lll}
\hline $\begin{array}{l}\text { Protected against contam- } \\
\text { ination? }\end{array}$ & Low risk & $\begin{array}{l}\text { Judgement comment: it is unlikely that the control group received the tele- } \\
\text { phone coaching intervention }\end{array}$ \\
\hline $\begin{array}{l}\text { Free from selective out- } \\
\text { come reporting? }\end{array}$ & Unclear risk & $\begin{array}{l}\text { Judgement comment: trial retrospectively registered and so not possible to } \\
\text { assess }\end{array}$ \\
\hline Other risks of bias? & Low risk & Judgement comment: no evidence of other risks of bias \\
\hline
\end{tabular}

Vidal-Pardo 2013

\begin{tabular}{ll}
\hline Methods & Study aim: to evaluate the effect of an educational intervention among primary care physicians on \\
several indicators of good clinical practice in diabetes care
\end{tabular}

Study design: cluster-RCT

\section{Participants}

Country: Spain

Setting: primary care physicians in Galicia (north-west Spain)

Number of clusters: 108

Number of providers: 108

Total number of patients: 2938

Percentage male: $52.4 \%$

Diabetes type: type 2

Average age (SD): NR

Inclusion criteria: patients aged $\geq 40$ years with more than 1 year of diagnosis of type 2 diabetes

Exclusion criteria: women with gestational diabetes

Interventions

Intervention (58 clusters, $\mathbf{n}=1437$ participants): educational intervention comprising (a) distribution of educational materials; (b) physicians' specific bench-marking information (audit and feedback); (c) an on-line course and 3 on-site educational workshops on diabetes.

Comparator (50 clusters, $\mathbf{n}=\mathbf{1 5 0 1}$ participants): usual care (not specified)

Duration: 6 months

Outcomes Primary outcome: measurement of risk factors (HbA1c; BP; LDL cholesterol); processes of care including annual eye examination

\section{Secondary outcomes: NR}

Baseline screening attendance (control group): $25.1 \%$

Sources of funding: unrestricted grant from Merck Sharp \& Dohme (MSD) and the Fundacion Escola Galega de Administracion Sanitaria (FEGAS). 
Vidal-Pardo 2013 (Continued)

Declaration of interest: none declared

\title{
Risk of bias
}

\begin{tabular}{|c|c|c|}
\hline Bias & Authors' judgement & Support for judgement \\
\hline $\begin{array}{l}\text { Adequate sequence gener- } \\
\text { ation? }\end{array}$ & Unclear risk & Not reported \\
\hline $\begin{array}{l}\text { Adequate allocation con- } \\
\text { cealement? }\end{array}$ & Low risk & $\begin{array}{l}\text { Judgement comment: unit of allocation at the level of the primary care physi- } \\
\text { cian and allocation performed prior to the start of the study }\end{array}$ \\
\hline $\begin{array}{l}\text { Similar baseline outcome } \\
\text { measurements? }\end{array}$ & Low risk & $\begin{array}{l}\text { Judgement comment: similar rates of eye examinations between arms at } \\
\text { baseline (Table } 3 \text { p 755) }\end{array}$ \\
\hline \multirow[t]{2}{*}{$\begin{array}{l}\text { Similar baseline character- } \\
\text { istics? }\end{array}$} & Low risk & $\begin{array}{l}\text { Quote: "Table } 2 \text { compares the groups of patients. Differences between the in- } \\
\text { tervention and control groups are slight and not statistically significant, ex- } \\
\text { cept for some variables at baseline such as family history of ischaemic heart } \\
\text { disease, personal history of prior coronary revascularisation, presence of neu- } \\
\text { ropathy and insulin use." p } 753\end{array}$ \\
\hline & & $\begin{array}{l}\text { Judgement comment: small baseline differences unlikely to influence out- } \\
\text { come }\end{array}$ \\
\hline $\begin{array}{l}\text { Incomplete outcome data } \\
\text { addressed? }\end{array}$ & Low risk & Judgement comment: low attrition and balanced between study arms \\
\hline $\begin{array}{l}\text { Knowledge of allocated in- } \\
\text { tervention prevented? }\end{array}$ & Unclear risk & Not reported \\
\hline $\begin{array}{l}\text { Protected against contam- } \\
\text { ination? }\end{array}$ & High risk & $\begin{array}{l}\text { Judgement comment: possibility of contamination as control and intervention } \\
\text { physicians worked in the same healthcare system. }\end{array}$ \\
\hline $\begin{array}{l}\text { Free from selective out- } \\
\text { come reporting? }\end{array}$ & Unclear risk & $\begin{array}{l}\text { Judgement comment: no protocol or trial registry entry available and there- } \\
\text { fore not possible to assess }\end{array}$ \\
\hline Other risks of bias? & Low risk & Judgement comment: no evidence of other sources of bias \\
\hline
\end{tabular}

Wagner 2001

Methods

Study aim: to evaluate the impact of primary care group visits (chronic care clinics) on the process and outcome of care for diabetic patients

Study design: cluster-RCT

Participants

\author{
Country: USA \\ Number of clusters: 35 \\ Number of providers: NR \\ Total number of patients: 707 \\ Percentage male: $53.4 \%$ \\ Diabetes type: NR
}

Setting: primary care clinics in the Group Health Cooperative in western Washington 
Wagner 2001 (Continued)

Average age (SD): 60.7 yrs (NR)

Inclusion criteria: all diabetic patients $\geq 30$ yrs of age

Exclusion criteria: patients who were terminally ill, demented or psychotic, or otherwise not able to participate in the study

Interventions

Intervention (14 clusters, $\mathbf{n}=\mathbf{2 7 8}$ participants): participants invited to attend a half-day chronic care clinic at their primary care clinic in groups of approx. 8 diabetic patients at intervals of $3-6$ months.

Each chronic care clinic group visit consisted of: individual visits with the primary care physician, nurse, and clinical pharmacist; and a group educational/ peer support session. Self-management support was also provided through one-on-one counselling with the practice nurse

Comparator ( 21 clusters, $\mathbf{n}=\mathbf{4 2 9}$ participants): usual care (not specified)

Duration: 24 months

Outcomes

Primary outcome: processes of diabetes care and satisfaction of intervention and control patients at baseline and at 24 months

Secondary outcomes: HRQOL using the SF36

Baseline screening attendance (control group): $62.2 \%$

\begin{tabular}{ll}
\hline Notes & Date conducted: NR \\
& Trial registration number: NR \\
& Sources of funding: Robert Wood Johnson Foundation \\
& Declaration of interest: NR
\end{tabular}

\section{Risk of bias}

\begin{tabular}{|c|c|c|}
\hline Bias & Authors' judgement & Support for judgement \\
\hline $\begin{array}{l}\text { Adequate sequence gener- } \\
\text { ation? }\end{array}$ & Unclear risk & Not reported \\
\hline $\begin{array}{l}\text { Adequate allocation con- } \\
\text { cealement? }\end{array}$ & Low risk & $\begin{array}{l}\text { Judgement comment: unit of allocation by primary care practice and alloca- } \\
\text { tion performed prior to the start of the study }\end{array}$ \\
\hline $\begin{array}{l}\text { Similar baseline outcome } \\
\text { measurements? }\end{array}$ & Low risk & Judgement comment: similar \% of baseline retinal exams across arms \\
\hline $\begin{array}{l}\text { Similar baseline character- } \\
\text { istics? }\end{array}$ & Low risk & $\begin{array}{l}\text { Quote: "Table } 1 \text { shows that there were no significant demographic, treatment, } \\
\text { or health status differences between groups." p } 697\end{array}$ \\
\hline & & $\begin{array}{l}\text { Judgement comment: imbalance in missing data could have influenced out- } \\
\text { come }\end{array}$ \\
\hline
\end{tabular}

Knowledge of allocated in- Unclear risk Not reported

tervention prevented?

Protected against contam- Low risk ination?

Judgement comment: control group unlikely to have received the intervention 
Wagner 2001 (Continued)

Free from selective out- Unclear risk Judgement comment: no protocol or trial registry entry available and therecome reporting? fore not possible to assess

Other risks of bias?

Walker 2008

Methods Study aim: to study the impact of a tailored telephone intervention compared to a standard print intervention on screening for diabetic retinopathy in an urban minority population

Study design: parallel-group RCT

Participants Country: USA

Setting: 3 inner city health centres

Total number of participants: 635

Percentage male: $39.5 \%$

Diabetes type: NR

Average age (SD): 56.6 yrs (12.5)

Inclusion criteria: aged > 18 years, diagnosed with diabetes, able to speak and read (or be read to in) English or Spanish, capable of providing informed consent, have access to a telephone, and report not having had a dilated fundus examination in the previous 12 months

Exclusion criteria: no access to a telephone; unable to speak English or Spanish; fundus examination in the previous 12 months

Interventions

Intervention ( $\mathbf{n}=\mathbf{3 2 6}$ ): tailored telephone intervention to promote retinopathy screening (up to 7 calls over a 6-month period). Participants were interviewed to identify issues and barriers that might either motivate them or prevent them from going for a dilated fundus examination. Attempts were made to engage all participants with targeted self-management strategies and dilated fundus examination education, and they were encouraged to make a screening appointment if they indicated they were ready to change.

Comparator $(\mathbf{n}=\mathbf{3 0 9})$ : participants were sent a printed booklet on preventing diabetic eye problems

Duration: 6 months

Outcomes Primary outcome: documentation of a dilated fundus examination within 6 months of randomisation

Secondary outcomes: factors that contribute to receiving a dilated fundus examination within 6 months for participants in the tailored telephone intervention. HbAlc results, from a 1-year period encompassing the participant's 6 -month intervention period

Baseline screening attendance (control group): $0 \%$

Notes

Date conducted: 2001 to 2005

Trial registration number: NR

Sources of funding: National Institute of Health, Rockerfeller Foundation

Declaration of interest: none declared

\section{Risk of bias}


Walker 2008 (Continued)

\begin{tabular}{|c|c|c|}
\hline Bias & Authors' judgement & Support for judgement \\
\hline $\begin{array}{l}\text { Adequate sequence gener- } \\
\text { ation? }\end{array}$ & Unclear risk & Not reported \\
\hline $\begin{array}{l}\text { Adequate allocation con- } \\
\text { cealement? }\end{array}$ & Unclear risk & Not reported \\
\hline $\begin{array}{l}\text { Similar baseline outcome } \\
\text { measurements? }\end{array}$ & Unclear risk & Not reported \\
\hline $\begin{array}{l}\text { Similar baseline character- } \\
\text { istics? }\end{array}$ & Low risk & $\begin{array}{l}\text { Quote: "There were no significant differences between the two study groups } \\
\text { on any characteristics." p } 188\end{array}$ \\
\hline $\begin{array}{l}\text { Incomplete outcome data } \\
\text { addressed? }\end{array}$ & Low risk & $\begin{array}{l}\text { Judgement comment: proportion of missing data low and balanced between } \\
\text { intervention and control groups }\end{array}$ \\
\hline $\begin{array}{l}\text { Knowledge of allocated in- } \\
\text { tervention prevented? }\end{array}$ & Low risk & $\begin{array}{l}\text { Quote: "The trained chart auditor was masked to the subjects' group assign- } \\
\text { ment." p } 186\end{array}$ \\
\hline $\begin{array}{l}\text { Protected against contam- } \\
\text { ination? }\end{array}$ & Low risk & $\begin{array}{l}\text { Judgement comment: it is unlikely that the control group received the tailored } \\
\text { telephone intervention }\end{array}$ \\
\hline $\begin{array}{l}\text { Free from selective out- } \\
\text { come reporting? }\end{array}$ & Unclear risk & $\begin{array}{l}\text { Judgement comment: no protocol or trial registry entry available and there- } \\
\text { fore not possible to assess }\end{array}$ \\
\hline Other risks of bias? & Low risk & Judgement comment: no evidence of other risks of bias \\
\hline
\end{tabular}

Ward 1996

Methods Study aim: to evaluate the impact of audit and feedback to general practitioners on the quality of their management of type 2 diabetes

Study design: cluster-RCT

\section{Participants}

Country: Australia

Setting: Western Australia metropolitan general practices

Number of clusters: 139

Number of providers: 139

Total number of patients: 386

Percentage male: NR

Diabetes type: type 2

Average age (SD): NR

Inclusion criteria: NR

Exclusion criteria: NR

Interventions

Intervention (doctor interview) (clusters NR, $\mathbf{n}=\mathbf{1 3 0}$ participants): each doctor was sent data by post on their management of patients compared to those of all doctors on the project along with a rec- 
Ward 1996 (Continued)

ommended standard. This was followed by an interview with an academic general practitioner to discuss their results using an interview proforma

Intervention (nurse interview) (clusters NR, $\mathbf{n}=\mathbf{1 2 1}$ participants): in addition to receiving their postal data, the doctor was interviewed by a state registered nurse to discuss their results using the same interview proforma

Comparator (no interview)(clusters NR, $\mathbf{n}=135$ participants): each doctor was sent their data by post only

Duration: 12 months

Outcomes

Primary outcome: 21 process outcomes on the Diabetic Healthcare Checklist (DHC), including eye examination (or referral to an ophthalmologist)

Secondary outcomes: NR

Baseline screening attendance (control group): $29.6 \%$

\begin{tabular}{|c|c|c|}
\hline Notes & \multicolumn{2}{|c|}{$\begin{array}{l}\text { Date conducted: NR } \\
\text { Trial registration number: NR } \\
\text { Sources of funding: NR } \\
\text { Declaration of interest: NR }\end{array}$} \\
\hline \multicolumn{3}{|l|}{ Risk of bias } \\
\hline Bias & Authors' judgement & Support for judgement \\
\hline $\begin{array}{l}\text { Adequate sequence gener- } \\
\text { ation? }\end{array}$ & Unclear risk & Not reported \\
\hline $\begin{array}{l}\text { Adequate allocation con- } \\
\text { cealement? }\end{array}$ & Low risk & $\begin{array}{l}\text { Judgement comment: unit of allocation by general practice and allocation } \\
\text { performed prior to the start of the study }\end{array}$ \\
\hline $\begin{array}{l}\text { Similar baseline outcome } \\
\text { measurements? }\end{array}$ & High risk & $\begin{array}{l}\text { Judgement comment: baseline differences in annual eye exams ( } 29.6 \% \text { com- } \\
\text { parator group, } 23.1 \% \text { doctor interview group, } 19.8 \% \text {, nurse interview group). } \\
\text { See Table } 1 \text { p } 145\end{array}$ \\
\hline $\begin{array}{l}\text { Similar baseline character- } \\
\text { istics? }\end{array}$ & Unclear risk & $\begin{array}{l}\text { Judgement comment: unclear if baseline differences in process of care influ- } \\
\text { ence outcome }\end{array}$ \\
\hline $\begin{array}{l}\text { Incomplete outcome data } \\
\text { addressed? }\end{array}$ & Low risk & Judgement comment: data from all participants available for analysis \\
\hline $\begin{array}{l}\text { Knowledge of allocated in- } \\
\text { tervention prevented? }\end{array}$ & High risk & $\begin{array}{l}\text { Judgement comment: } 1 \text { of the outcome assessors was the research nurse who } \\
\text { conducted the nurse interviews in } 1 \text { arm of the trial and was therefore un- } \\
\text { masked }\end{array}$ \\
\hline $\begin{array}{l}\text { Protected against contam- } \\
\text { ination? }\end{array}$ & Low risk & Judgement comment: control group unlikely to have received the intervention \\
\hline $\begin{array}{l}\text { Free from selective out- } \\
\text { come reporting? }\end{array}$ & Unclear risk & $\begin{array}{l}\text { Judgement comment: no protocol or trial registry entry available and there- } \\
\text { fore not possible to assess }\end{array}$ \\
\hline Other risks of bias? & Low risk & Judgement comment: no evidence of other sources of bias \\
\hline
\end{tabular}


Weiss 2015

$\begin{array}{ll}\text { Methods } & \begin{array}{l}\text { Study aim: to test the impact of a home-based behavioural activation programme to improve rates of } \\ \text { dilated fundus examinations in older African Americans with diabetes }\end{array} \\ \text { Study design: parallel-group RCT } \\ \text { Country: USA } \\ \text { Setting: } 2 \text { urban medical centres } \\ \text { Total number of participants: } 206 \\ \text { Percentage male: } 39.5 \% \\ \text { Diabetes type: type } 2 \\ \text { Average age (SD): } 72.7 \text { yrs (6.2) } \\ \text { Inclusion criteria: aged } \geq 65 \text { years, self-identification as an African-American individual, diagnosis of } \\ \text { type } 2 \text { diabetes mellitus, no self-report or medical documentation of a dilated fundus examination in } \\ \text { the past } 12 \text { months, and access to a telephone } \\ \text { Exclusion criteria: cognitive impairment (based on an abbreviated version of the Mini-Mental State Ex- } \\ \text { amination), current significant psychiatric disorder, current medical disorder limiting life expectancy, } \\ \text { need for dialysis, and hearing impairment that precluded research participation }\end{array}$

Interventions

Intervention ( $\mathbf{n}=\mathbf{1 0 3}$ ): behavioural intervention delivered by specially-trained community health worker. Intervention consisted of education, identifying barriers to a dilated fundus examination and action-planning

Comparator $(\mathbf{n}=\mathbf{1 0 3})$ : supportive therapy only without educational materials or behavioural strategies or goal-setting

Duration: 6 months

Outcomes Primary outcome: medical documentation of a dilated fundus examination by the 6-month follow-up visit

Secondary outcomes: risk perceptions of diabetes, diabetes self-care behaviours, depressive symptoms

Baseline screening attendance (control group): $0 \%$

Notes Date conducted: October 2010 to May 2013

Trial registration number: NCT01179555

Sources of funding: Pennsylvania Department of Health

Declaration of interest: none declared

\section{Risk of bias}

\begin{tabular}{lll}
\hline Bias & Authors' judgement & Support for judgement \\
\hline $\begin{array}{l}\text { Adequate sequence gener- } \\
\text { ation? }\end{array}$ & Low risk & $\begin{array}{l}\text { Quote: "..participants who completed the baseline assessment were random- } \\
\text { ized using random permuted blocks with a } 1 \text { to } 1 \text { allocation ratio to BADRP or } \\
\text { supportive therapy (ST)." p } 1006\end{array}$ \\
\hline $\begin{array}{l}\text { Adequate allocation con- } \\
\text { cealement? }\end{array}$ & Low risk & $\begin{array}{l}\text { Quote: "Randomization sheets were stored in sequentially numbered sealed } \\
\text { envelopes that were opened by the project director after each participant } \\
\text { completed baseline assessment." p 1006 }\end{array}$ \\
\hline \hline
\end{tabular}


Weiss 2015 (Continued)

Similar baseline outcome Unclear risk Not reported
measurements?

Similar baseline character- Low risk istics?

Quote: "The 2 arms were balanced with respect to age, education, sex, recruitment site, and marital status. Differences on the Risk Perceptions and Risk Knowledge Survey of Diabetes Mellitus, Diabetes Self-Care Inventory, Patient Health Questionnaire, Literacy Assessment for Diabetes, and the NEI-VFQ 25 composite scores that may have influenced the primary outcome were not identified. Participants in the BADRP group had lower HbAlc levels and chronic disease scores at baseline."

p 1008

Incomplete outcome data Low risk
addressed?

Judgement comment: attrition (approx. 10\%) balanced across groups and reasons for exclusion given (see CONSORT diagram $\mathrm{p}$ 1008)

Knowledge of allocated in- Low risk tervention prevented?

Quote: "Follow-up assessments were conducted in participants' homes at 6 months' follow-up by community health workers masked to treatment assignment." p 1007

\begin{tabular}{lll}
\hline $\begin{array}{l}\text { Protected against contam- } \\
\text { ination? }\end{array}$ & Low risk & $\begin{array}{l}\text { Judgement comment: it is unlikely that the control group received the behav- } \\
\text { ioural intervention }\end{array}$ \\
\hline $\begin{array}{l}\text { Free from selective out- } \\
\text { come reporting? }\end{array}$ & High risk & $\begin{array}{l}\text { Judgement comment: per-protocol analysis. Participants who had not re- } \\
\text { ceived the intervention were excluded from the analysis }\end{array}$ \\
\hline $\begin{array}{ll}\text { Other risks of bias? } & \text { Low risk }\end{array}$ & Judgement comment: no evidence of other risks of bias \\
\hline
\end{tabular}

Welch 2011

\begin{tabular}{ll}
\hline Methods & Study aim: to evaluate the clinical usefulness of a nurse-led diabetes care programme for poorly-con- \\
trolled Hispanic type 2 diabetes patients
\end{tabular}
trolled Hispanic type 2 diabetes patients

Study design: parallel-group RCT

Participants

\section{Country: USA}

Setting: a single urban community healthcare centre in Springfield, Massachusetts.

Total number of patients: 46

Percentage male: $33 \%$

Diabetes type: type 2

Average age (SD): 55.8 yrs (10)

Inclusion criteria: duration of type 2 diabetes of at least 1 year based on medical record review and treatment history; age $30-85$ years; $\mathrm{HbAlc}>7.5 \%$ within the past 3 months but not $>14 \%$; Hispanic ethnicity; independently living and ambulatory

Exclusion criteria: severe diabetes complications, severe psychiatric illness, or severe visual restrictions, or would not be available for the study period (e.g. leaving the area, pregnant or planning to become pregnant)

Interventions

Intervention ( $\mathbf{n}=\mathbf{2 5}$ ): 7 x 1-hour diabetes care visits over a 12-month period conducted by a bicultural/bilingual diabetes nurse and dietician team (both certified diabetes educators). Use of CDMP diabetes care management software that provides tools for continuous care and contact between patients and their providers. Participants in the intervention group also received diabetes eye screening using 
Welch 2011 (Continued)

the Diabetes Eye Care and Treatment (DECAT) programme using the clinically-validated Joslin Vision Network (JVN) protocol

Comparator ('attention control') $(\mathbf{n}=\mathbf{2 1})$ : diabetes education interventionconsisting of $7 \times 1$-hour visits over a 12-month period conducted by bicultural/bilingual clinic support staff who also encouraged participants to formulate diabetes-related questions for discussion with their primary care provider at the next scheduled primary care visit

Duration: 12 months

Outcomes

Primary outcome: adherence to national clinical practice guidelines (blood glucose, blood pressure, foot exam, eye exam), and levels of diabetes distress, depression, and treatment satisfaction

Secondary outcomes: NR

Baseline screening attendance (control group): NR

\begin{tabular}{ll}
\hline Notes & Date conducted: NR \\
& Trial registration number: NR \\
& Sources of funding: Baystate Medical Center Academic Affairs Internal Research Grant \\
& Declaration of interest: NR
\end{tabular}

\section{Risk of bias}

\begin{tabular}{|c|c|c|}
\hline Bias & Authors' judgement & Support for judgement \\
\hline $\begin{array}{l}\text { Adequate sequence gener- } \\
\text { ation? }\end{array}$ & Low risk & $\begin{array}{l}\text { Quote: "Participants were randomly assigned to the CDMP intervention group } \\
\text { (IC) or the attention control group (AC) by a fair coin toss." p } 682\end{array}$ \\
\hline $\begin{array}{l}\text { Adequate allocation con- } \\
\text { cealement? }\end{array}$ & Unclear risk & Not reported \\
\hline $\begin{array}{l}\text { Similar baseline outcome } \\
\text { measurements? }\end{array}$ & Unclear risk & Not reported \\
\hline $\begin{array}{l}\text { Similar baseline character- } \\
\text { istics? }\end{array}$ & Low risk & $\begin{array}{l}\text { Quote: "There were no differences between groups at baseline except for mari- } \\
\text { tal status }(P=.04) \text { (Table } 1) . " \text { p } 684\end{array}$ \\
\hline $\begin{array}{l}\text { Incomplete outcome data } \\
\text { addressed? }\end{array}$ & Low risk & Judgement comment: low attrition and balanced between study arms \\
\hline $\begin{array}{l}\text { Knowledge of allocated in- } \\
\text { tervention prevented? }\end{array}$ & Unclear risk & $\begin{array}{l}\text { Judgement comment: not clear whether eye-screening outcome assessors } \\
\text { were masked }\end{array}$ \\
\hline $\begin{array}{l}\text { Protected against contam- } \\
\text { ination? }\end{array}$ & High risk & $\begin{array}{l}\text { Quote : "the diabetes educators in the intervention condition trained and su- } \\
\text { pervised the attention control clinical staff." p } 687\end{array}$ \\
\hline $\begin{array}{l}\text { Free from selective out- } \\
\text { come reporting? }\end{array}$ & Unclear risk & $\begin{array}{l}\text { Judgement comment: no protocol or trial registry entry available and there- } \\
\text { fore not possible to assess }\end{array}$ \\
\hline Other risks of bias? & Low risk & Judgement comment: no evidence of other sources of bias \\
\hline
\end{tabular}


Zangalli 2016

Methods

Study aim: to evaluate the effectiveness of a multifaceted intervention with personal communication to improve dilated fundus examination follow-up adherence among those who are less likely to adhere

Study design: parallel-group RCT
Country: USA

Setting: tertiary eye clinic

Total number of participants: 522

Percentage male: $34 \%$

Diabetes type: NR

Average age (SD): 61 yrs (13.0)

Inclusion criteria: eligible participants were > 18 years of age; had no, mild, or moderate DR; were recommended for a follow-up dilated fundus examination; and had not previously scheduled a follow-up visit

\section{Exclusion criteria: NR}

Intervention ( $\mathbf{n} \mathbf{2 6 2}$ ): intervention group received a personalised reminder letter with a 1-page brochure about diabetic retinopathy 1 month prior to the recommended visit. 2 weeks later, a research assistant called participants to offer personal assistance with scheduling an appointment. For participants who made an appointment, a reminder letter was mailed 3 weeks prior to the scheduled appointment. Participants also received automated reminder calls the day before the scheduled appointment

Comparator $(\mathbf{n}=\mathbf{2 6 0}$ ): usual care (consisting of participants receiving a reminder letter 1 month prior to the recommended follow-up date. Participants received no active assistance with scheduling appointments. Participants who made appointments received automated reminder calls the day before scheduled appointments)

Duration: 6 months
Primary outcome: attendance at a follow-up appointment within 3 months of suggested return date

Secondary outcomes: barriers to care use

Baseline screening attendance (control group): NR

Notes Date conducted: April to October 2012

Trial registration number: NR

Sources of funding: Centers for Disease Control and Prevention

Declaration of interest: none declared

\section{Risk of bias}

\begin{tabular}{|c|c|c|}
\hline Bias & Authors' judgement & Support for judgement \\
\hline $\begin{array}{l}\text { Adequate sequence gener- } \\
\text { ation? }\end{array}$ & Low risk & $\begin{array}{l}\text { Quote: "Participants were randomized to usual care or intervention within age } \\
\text { strata ( } \geq 65 \text { and }<65 \text { years) using the method of random permuted blocks with } \\
\text { block sizes of } 2,4 \text {, and } 6 \text {." p } 2\end{array}$ \\
\hline $\begin{array}{l}\text { Adequate allocation con- } \\
\text { cealement? }\end{array}$ & Unclear risk & Not reported \\
\hline
\end{tabular}


Zangalli 2016 (Continued)

Similar baseline outcome Unclear risk Not reported measurements?

Similar baseline character- Low risk istics?
Quote: "Participants in the intervention and control groups had similar baseline characteristics with regard to sex, ethnicity, and age." p 3

Incomplete outcome data Low risk Judgement comment: low attrition and balanced across groups
addressed?

addressed?

Knowledge of allocated in- Unclear risk Not reported
tervention prevented?

tervention prevented?

\begin{tabular}{ll}
\hline $\begin{array}{l}\text { Protected against contam- Low risk } \\
\text { ination? }\end{array}$ & $\begin{array}{l}\text { Judgement comment: it is unlikely that the control group received the inter- } \\
\text { vention }\end{array}$
\end{tabular}

Protected against contam- Low risk
ination?

\begin{tabular}{ll}
\hline $\begin{array}{l}\text { Free from selective out- } \\
\text { come reporting? }\end{array}$ & Unclear risk \\
\end{tabular}

\begin{tabular}{ll}
\hline Other risks of bias? $\quad$ Low risk Judgement comment: no evidence of other risks of bias \\
\hline
\end{tabular}

Zwarenstein 2014

Methods Study aim: to evaluate the effectiveness of printed educational messages aimed at family doctors on
rates of retinal screening attendance amongst patients with diabetes

Study design: cluster-RCT

\begin{tabular}{ll}
\hline Participants & Country: Canada \\
Setting: Primary care (family physicians)
\end{tabular}

Total number of clusters: 4282

Number of providers: 5048

Total number of patients: 179,833

Percentage male: $51.2 \%$

Diabetes type: NR

Average age (SD): $61.7 \mathrm{yrs}(13.1)$

Inclusion criteria: patients diagnosed with diabetes who were at least 30 years old and visited 1 of the target family practitioners within 1 year of the intervention mail-out

Exclusion criteria: patients who had already had an eye examination in the 9 months immediately prior to the office visit

Interventions Alternative printed educational messages (PEM) containing prompts to encourage diabetic retinopathy
screening were mailed to each family physician in conjunction with a widely-read professional newslet-
ter (Informed)

Intervention arm 1 (1066 clusters): PEM consisting of a 2-page insert, indistinguishable from the rest of Informed in size and style (the 'insert'). The insert contained a concise summary of an evidence-based guideline and references

Intervention arm 2 (535 clusters): (PEM) consisting of a short directive message on a postcard-sized card ('outsert') stapled to the front page of Informed 
Zwarenstein 2014 (Continued)

Intervention arm 3 (536 clusters): PEM 'outsert' and supplied with a pad of sticky take-home reminders (aimed at patients, to remind them to make an appointment for an eye exam), to be given to participants

Intervention arm 4 (535 clusters): PEM 'insert' and 'outsert'

Intervention arm 5 (533 clusters): PEM 'insert' and 'outsert' and take-home reminders

Comparator (1077 clusters): newsletter without the PEM

Duration: 3 months

Outcomes Primary outcome: whether or not an eligible trial patient received an eye exam within 90 days of their first family practitioner visit.

Secondary outcomes: the impact of patient age on the uptake of eye exams

Baseline screening attendance (control group): NR

Notes Date conducted: 2005 to 2006

Trial registration number: NCT00210275

Sources of funding: Canadian Institutes for Health Research, Institute for Clinical Evaluation Sciences

Declaration of interest: none declared

Study protocol has been published: www.ncbi.nlm.nih.gov/pubmed/18039361

\section{Risk of bias}

\begin{tabular}{|c|c|c|}
\hline Bias & Authors' judgement & Support for judgement \\
\hline $\begin{array}{l}\text { Adequate sequence gener- } \\
\text { ation? }\end{array}$ & Low risk & $\begin{array}{l}\text { Quote: "Practices were randomly assigned to an intervention group by the } \\
\text { study statistician, using computer generated random numbers." p } 2\end{array}$ \\
\hline $\begin{array}{l}\text { Adequate allocation con- } \\
\text { cealement? }\end{array}$ & Low risk & $\begin{array}{l}\text { Judgement comment:unit of allocation by GP practice and allocation per- } \\
\text { formed prior to the start of the study }\end{array}$ \\
\hline $\begin{array}{l}\text { Similar baseline outcome } \\
\text { measurements? }\end{array}$ & Unclear risk & Not reported \\
\hline $\begin{array}{l}\text { Similar baseline character- } \\
\text { istics? }\end{array}$ & Low risk & $\begin{array}{l}\text { Quote: "There were small, clinically unimportant, differences between the de- } \\
\text { mographics of patients with diabetes who paid a visit to a study physician and } \\
\text { those who did not, and between those who were and were not included in the } \\
\text { analysis (Table 2)." p } 5\end{array}$ \\
\hline $\begin{array}{l}\text { Incomplete outcome data } \\
\text { addressed? }\end{array}$ & Low risk & Judgement comment: data from all clusters reported \\
\hline $\begin{array}{l}\text { Knowledge of allocated in- } \\
\text { tervention prevented? }\end{array}$ & Low risk & Judgement comment: outcomes were obtained from routinely-collected data \\
\hline $\begin{array}{l}\text { Protected against contam- } \\
\text { ination? }\end{array}$ & Low risk & $\begin{array}{l}\text { Judgment comment: allocation by cluster and unlikely that the control group } \\
\text { received the intervention }\end{array}$ \\
\hline $\begin{array}{l}\text { Free from selective out- } \\
\text { come reporting? }\end{array}$ & Low risk & $\begin{array}{l}\text { Judgement comment: reported outcomes consistent with trial registry } \\
\text { NCT00210275 }\end{array}$ \\
\hline Other risks of bias? & Low risk & Judgement comment: no evidence of other risks of bias \\
\hline
\end{tabular}


ADA: American Diabetes Association

ADAP: Annual Diabetes Assessment Program

BMI: body mass index

BP: blood pressure

$\mathrm{CHW}$ : community health worker

DR: diabetic retinopathy

HbA1c: glycaemic haemoglobin

HCC: hierarchical condition category

HMO: Health Maintenance Organisation

HRQOL: health-related quality of life

LDL: low-density lipoprotein

QI: quality improvement

SBP: systolic blood pressure

Characteristics of excluded studies [ordered by study ID]

\begin{tabular}{|c|c|}
\hline Study & Reason for exclusion \\
\hline Abraira 2003 & No data on retinopathy screening attendance \\
\hline Aleo 2015 & No data on retinopathy screening attendance \\
\hline Alfadda 2011 & Not RCT \\
\hline Anderson 2003a & Not RCT \\
\hline Anderson 2010 & No data on retinopathy screening attendance \\
\hline Arora 2014 & No data on retinopathy screening attendance \\
\hline Bellazzi 2004 & No data on retinopathy screening attendance \\
\hline Denig 2014 & No data on retinopathy screening attendance \\
\hline Gangwar 2014 & No data available on control group (contacted author) \\
\hline Gary 2004 & No data on retinopathy screening attendance \\
\hline Harris 2013 & Not RCT \\
\hline Hazavehei 2010 & Evaluated intentions to attend for retinopathy screening rather than attendance \\
\hline Hollander 2005 & Not RCT \\
\hline Jones 2006 & Not RCT \\
\hline Kuvaja-Kollner 2013 & Not RCT \\
\hline Lewis 2007 & Qualitative study. No data on retinopathy screening attendance \\
\hline Maberley 2003 & Health economic paper. No data on retinopathy screening attendance \\
\hline Mangione 2006 & Not RCT \\
\hline Mazzuca 1988 & No data on retinopathy screening attendance \\
\hline McCulloch 1998 & Not RCT \\
\hline
\end{tabular}




\begin{tabular}{|c|c|}
\hline Study & Reason for exclusion \\
\hline Montori 2002 & Not RCT \\
\hline Montori 2004 & Not RCT \\
\hline Peters 1998 & Not RCT \\
\hline Polak 2003 & Health economic paper. No data on retinopathy screening attendance \\
\hline Rees 2013 & No data on retinopathy screening attendance \\
\hline Samoutis 2010 & Not RCT \\
\hline Schectman 2004 & Not RCT \\
\hline Shah 2014 & No data on retinopathy screening attendance \\
\hline Shea 2006 & No data on retinopathy screening attendance \\
\hline Solorio 2015 & Not RCT \\
\hline Thoolen 2008 & No data on retinopathy screening attendance \\
\hline Wagner 2008 & Knowledge of diabetic retinopathy rather than attendance \\
\hline Weston 2008 & Used vignettes rather than real patients \\
\hline Young 2014 & No data on retinopathy screening attendance \\
\hline
\end{tabular}

Characteristics of ongoing studies [ordered by study ID]

\section{ACTRN12614001110673}

\begin{tabular}{ll}
\hline Trial name or title & The diabetes and eye health project: increasing eye examinations for adults newly diagnosed with \\
type 2 diabetes.
\end{tabular}

\begin{tabular}{ll}
\hline Methods & Parallel-group RCT (Solomon four group design) \\
\hline Participants & $\begin{array}{l}\text { Inclusion criteria: diagnosed with type } 2 \text { diabetes in the past } 3 \text { years; Australian residents; able } \\
\text { to read English; registered with the National Diabetes Services Scheme (NDSS); } 1 \text { of either: young } \\
\text { adult (aged } 18 \text { - } 39 \text { years), or live in rural/regional locations of Victoria, Australia }\end{array}$
\end{tabular}

Interventions

Intervention: printed materials (leaflet) containing persuasive behaviour change messages designed to raise awareness of the importance of maintaining optimal blood glucose and blood pressure levels to minimise the risk of diabetic retinopathy, increase intentions to engage in regular eye examinations and increase self-reported eye examinations. The leaflet will be mailed on a single occasion to study participants.

Comparator: participants randomised to the usual screening group will be advised by their endocrinologist during their diabetes clinic visit to arrange an eye examination with their usual eye care professional (as in current standard of care)

Outcomes $\quad$ From www.anzctr.org.au/

Primary outcome: self-reported eye health examinations assessed by response to a single questionnaire item ("Since you were diagnosed with diabetes, have you had your eye health checked?"). 
ACTRN12614001110673 (Continued)

In order to minimise social desirability bias and any potential confounding influence of question-behaviour effect, the question will be embedded within a suite of standard self-management questions based on information already provided to all new National Diabetes Service Scheme registrants

Secondary outcomes: intention to seek eye health examinations assessed by summed response to 3 intention items designed specifically for this purpose

\begin{tabular}{ll}
\hline Starting date & September 2014 \\
\hline Contact information & $\begin{array}{l}\text { Prof Jane Speight, The Australian Centre for Behavioural Research in Diabetes, } 206 \text { Queensberry } \\
\text { Street, Melbourne, VIC 3000, Australia. +61 (0)38648 1844, jspeight@acbrd.org.au }\end{array}$ \\
\hline
\end{tabular}

Notes

\section{ISRCTN31439939}

Trial name or title

The Kilimanjaro Diabetic Programme: the development of a sustainable regional eye health screening programme to prevent blindness among diabetic patients due to diabetic retinopathy

\begin{tabular}{ll}
\hline Methods & Parallel-group RCT \\
\hline Participants & $\begin{array}{l}\text { Inclusion criteria: all known adult diabetic patients resident in Kilimanjaro region and attending a } \\
\text { diabetic clinic at Kilimanjaro Christian Medical Centre (KCMC) or at } 1 \text { of the district diabetic clinics } \\
\text { in the } 6 \text { rural districts of Kilimanjaro region }\end{array}$ \\
\hline
\end{tabular}

Interventions

Phase l:

Intervention group: a digital diabetic retinopathy screening camera will be placed in the diabetic clinic at KCMC

Control group: patients will be advised to go to the eye clinic at KCMC for a dilated screening examination by an ophthalmologist

All participants will receive 3 information leaflets on diabetic retinopathy and be counselled by the health workers in the diabetic clinic that they should have screening for diabetic retinopathy. Visual acuity measurement will be performed and dilating drops installed by the screening team

Phase II:

The retinopathy screening camera will go to all district diabetic clinics twice in the 6-month intervention period. Patients registered at these clinics will all be advised by clinic staff to attend for retinopathy screening. The intervention group will receive a text message by mobile phone advising them of the date of the screening and inviting them to come

From www.isrctn.com/
Primary outcome: uptake of screening for diabetic retinopathy
Secondary outcomes: prevalence of diabetic retinopathy in urban and rural diabetic patients in
Kilimanjaro region; prevalence of cataract in urban and rural diabetic patients in Kilimanjaro re-
gion


ISRCTN31439939 (Continued)

Notes

ISRCTN87561257

\begin{tabular}{ll}
\hline Trial name or title & Individual risk-based screening for diabetic retinopathy (ISDR) \\
\hline Methods & Parallel-group RCT \\
\hline Participants & Inclusion criteria: patients aged 12 or above who attend the community clinic for retinal screening
\end{tabular}

\begin{tabular}{ll}
\hline Interventions & Intervention: : personalised risk-based screening intervals \\
Comparator: annual screening intervals (usual care)
\end{tabular}

Outcomes From www.isrctn.com/

Primary outcome: comparison of attendance rates for follow-up screening in the 2 arms of the study (non-attendance will be defined as failure to attend 2 appointments for screening (usually within 6 weeks of each other))

Secondary outcomes: number of cases of STDR detected; retinopathy level at screening (Liverpool and NDESP grading); maculopathy level at screening (Liverpool and NDESP grading); number of false positive screening episodes; number of screening appointments; number of dedicated diabetes assessment clinic appointments; number of other eye appointments for diabetic eye disease; visual acuity (logMAR); new visual impairment ( $\geq+0.50 \log M A R)$; new visual impairment due to diabetic retinopathy ( $\geq+0.50 \log M A R)$; number of missed appointments to screening; patient acceptability measures (using a questionnaire designed for the trial); QALYs estimated using EQ-5D-5L and Health Utilities Index Mark 3 (HUI3); cost per QALY gained

Starting date November 2014 to January 2018
ment, Royal Liverpool University Hospital, Prescot Street, Liverpool, L7 8XP, UK

Notes

\section{NCT01212328}

$\begin{array}{ll}\text { Trial name or title } & \text { Improving diabetes care: multicomponent cardiovascular disease risk reduction strategies for peo- } \\ \text { ple with diabetes in South Asia - The CARRS Multi-center Translation Trial }\end{array}$

\begin{tabular}{ll}
\hline Methods & Parallel-group RCT \\
\hline Participants & Inclusion criteria: aged 35 years and older with a confirmed diagnosis of diabetes and poor gly- \\
& caemic control (as evidenced by HbAlc $\geq 8.0 \%$ ) and 1 or both of: dyslipidemia (LDL $\geq 130 \mathrm{mg} / \mathrm{dl}$ ) or \\
& SBP $\geq 140 \mathrm{mmHg}$, irrespective of lipid- or BP-lowering medication use \\
\hline
\end{tabular}

Interventions

Intervention: the participants will receive integrated diabetes care management consisting of current diabetes management guidelines and non-physician care co-ordinator assistance and electronic health records- decision-support software (EHR-DSS) (The software will generate diabetes management prompts for the treating physician and reminders for clinic visits for the intervention arm participants) 
Comparator: participants will continue with the usual diabetes care with no care co-ordinator assistance and no decision-support software management prompt

From clinicaltrials.gov/
Primary outcome: multiple CVD risk factor control targets (blood glucose and either blood pres-
sure or cholesterol, or all 3)
Secondary outcomes: single risk factor control of at least 1 target, either HbA1c or blood pressure
or LDL-cholesterol; process and patient-centered measures; cost-effectiveness analysis of the in-
tervention compared to usual care; prescriber and patient acceptability of the Digital Support soft-
ware and care cordinator with management guidelines

\begin{tabular}{ll}
\hline Starting date & October 2010 to June 2014 \\
\hline Contact information & Kavita Singh, MSc Tel: $+91-11-26850118$ ext 39 email;kavita@ccdcindia.org \\
\hline Notes & Trial protocol has been published: www.ncbi.nlm.nih.gov/pubmed/23084280 \\
\hline
\end{tabular}

\section{NCT01351857}

$\begin{array}{ll}\text { Trial name or title } & \begin{array}{l}\text { Diabetes care management compared to standard diabetes care in adolescents and young adults } \\ \text { with type } 1 \text { diabetes (TransClin) }\end{array}\end{array}$

\begin{tabular}{ll}
\hline Methods & Parallel-group RCT \\
\hline Participants & $\begin{array}{l}\text { Inclusion criteria: patients between the ages of } 17 \text { and } 20 \text { years with an established type } 1 \text { dia- } \\
\text { betes diagnosis for a minimum of } 1 \text { year }\end{array}$ \\
\hline
\end{tabular}

Interventions $\quad$ From clinicaltrials.gov/

Intervention: a certified diabetes educator will act as a 'Transition Co-ordinator' to provide transition support and the link between paediatric and adult diabetes care. The Transition Co-ordinator is central to the intervention and will provide ongoing contact with the medical system as well as education and clinical support where appropriate

Comparator: current standard of care (participants in the control group will transition to adult care equal to the intervention group and will differ only by exclusion of Transition Co-ordinator)

Outcomes Primary outcome: proportion of participants who fail to attend at least 1 outpatient adult endocrinology visit during the second year after transition to adult diabetes care

Secondary Outcomes: frequency of HbA1C measurement (in the 2-year transfer to adult care); frequency of retinal exam, microalbumin to creatinine ratio, fasting lipid profile and foot exam testing; rate of hospitalisation/ER visits for acute complications of diabetes

\begin{tabular}{ll}
\hline Starting date & April 2012 to April 2017 \\
\hline Contact information & Cheril Clarson, MD, London Health Sciences Centre Children's Hospital \\
\hline Notes & Trial protocol has been published: www.ncbi.nlm.nih.gov/pubmed/24106787 \\
\hline
\end{tabular}


NCT01837121 (Continued)

Methods $\quad$ Parallel group RCT

\begin{tabular}{ll}
\hline Participants & Inclusion criteria: patients with diabetes with access to a cell phone \\
\hline Interventions & $\begin{array}{l}\text { Intervention: patient will receive a SMS reminder message about the revisit time and venue } 1 \\
\text { week and } 1 \text { day before the appointment } \\
\text { Comparator: usual care }\end{array}$ \\
\hline
\end{tabular}

From clinicaltrials.gov/
Primary outcome: non-attendance rate
Secondary outcomes: knowledge about diabetic retinopathy; presenting vision in the better-see-
ing and worse-seeing eyes; vision Loss of 2+ lines of presenting vision in better-seeing eye thought
due to diabetic retinopathy; satisfaction with care; number of treatments received for diabetic
retinopathy

\begin{tabular}{ll}
\hline Starting date & April 2013 to June 2015 \\
\hline Contact information & $\begin{array}{l}\text { Nathan G Congdon MD MPH. Blindness Prevention and Treatment Department, Zhongshan Oph- } \\
\text { thalmic Center }\end{array}$ \\
\hline Notes & \\
\hline
\end{tabular}

\begin{tabular}{ll}
\hline Trial name or title & Incentives in diabetic eye assessment by screening (IDEAS) \\
\hline Methods & Parallel-group RCT \\
\hline
\end{tabular}

\begin{tabular}{ll}
\hline Participants & $\begin{array}{l}\text { Inclusion criteria: diabetic patients (> 16 years) who were invited to screening in the last } 24 \\
\text { months on a yearly basis and failed to attend or contact the screening service to rearrange an ap- } \\
\text { pointment }\end{array}$
\end{tabular}

Interventions Intervention ('Fixed Incentive'): Standard invitation letter from the screening service, with additional text offering a fixed financial incentive (GBP 10) if they attend screening

Intervention 'Probabilistic incentive': invitation letter from the screening service, with additional text offering a probabilistic financial incentive (entry into a lottery offering at least a 1 in 100 chance to win GBP 1000) if they attend screening

Comparator: standard intervention from the screening service

$\begin{array}{ll}\text { Outcomes } & \text { From clinicaltrials.gov/ } \\ & \text { Primary outcome: attendance at screening appointment at designated appointment date (be- } \\ \text { tween } 3 \text { months and } 1 \text { year) }\end{array}$

Secondary outcome: outcome from diabetic retinopathy screening

\begin{tabular}{ll}
\hline Starting date & March 2015 to January 2016 \\
\hline Contact information & Colin Bicknell, Clinical Senior Lecturer and Consultant Vascular Surgeon, Imperial College London \\
\hline Notes & $\begin{array}{l}\text { Trial protocol has been published: bmcophthalmol.biomedcentral.com/arti- } \\
\text { cles/10.1186/s12886-016-0206-4 }\end{array}$ \\
\hline
\end{tabular}




\begin{tabular}{ll}
\hline Methods & Parallel-group RCT \\
\hline Participants & $\begin{array}{l}\text { Inclusion criteria: patients with a known diagnosis of Type } 1 \text { diabetes for } \geq 5 \text { years or Type } 2 \text { dia- } \\
\text { betes of any duration with at least a 12-month interval since the last screening for diabetic eye dis- } \\
\text { ease by an eye-care professional }\end{array}$ \\
\hline
\end{tabular}

Interventions

Intervention: on-site screening. Participants randomised to the on-site screening group will be advised by their Endocrinologist during their diabetes clinic visit to arrange an eye examination with their usual eye-care professional (as in current standard of care). In addition they will also undergo:

- non-mydriatic ultra-widefield (UWF) retinal imaging on the same day as their diabetes clinic visit

- half of this group will by random allocation undergo optical coherence tomography (OCT) using the Zeiss Cirrus OCT, which may or may not be done on the same day (for practical reasons regarding availability of OCT at the hospital)

Comparator: usual screening. Participants randomised to the usual screening group will be advised by their endocrinologist during their diabetes clinic visit to arrange an eye examination with their usual eye-care professional (as in current standard of care)

Futcomes
Prom clinicaltrials.gov/
Secondary outcomes: screening adherence, determined by (i) the proportions of participants who
have screening completed within 12 months of randomisation by the primary screening
method, i.e. non-mydriatic UWF images (On-site Screening group) or an eye examination by an eye-
care professional (Usual Screening group); (ii) for participants in the Onsite Screening group, the
proportion who have also had a screening eye examination by an eye-care professional within 1
year of randomisation; proportion of participants with Diabetic Maculopathy (DME)

Starting date February 2016 to January 2019

\section{DATA AND ANALYSES}


Comparison 1. Any quality improvement intervention compared to usual care

\begin{tabular}{lllll}
\hline Outcome or subgroup title & No. of studies & $\begin{array}{l}\text { No. of partici- } \\
\text { pants }\end{array}$ & Statistical method & Effect size \\
\hline $\begin{array}{l}1 \text { Proportion of participants attending } \\
\text { screening }\end{array}$ & 56 & 329164 & $\begin{array}{l}\text { Risk Difference (M-H, Ran- } \\
\text { dom, 95\% Cl) }\end{array}$ & $0.12[0.10,0.14]$ \\
\hline $\begin{array}{l}1.1 \text { Intervention specifically targeting dia- } \\
\text { betic retinopathy screening }\end{array}$ & 13 & 118938 & $\begin{array}{l}\text { Risk Difference (M-H, Ran- } \\
\text { dom, 95\% Cl) }\end{array}$ & $0.17[0.11,0.22]$ \\
\hline $\begin{array}{l}1.2 \text { General intervention to improve the } \\
\text { quality of diabetes care }\end{array}$ & 43 & 210226 & $\begin{array}{l}\text { Risk Difference (M-H, Ran- } \\
\text { dom, 95\% Cl) }\end{array}$ & $0.12[0.09,0.15]$ \\
\hline
\end{tabular}

Analysis 1.1. Comparison 1 Any quality improvement intervention compared to usual care, Outcome 1 Proportion of participants attending screening.

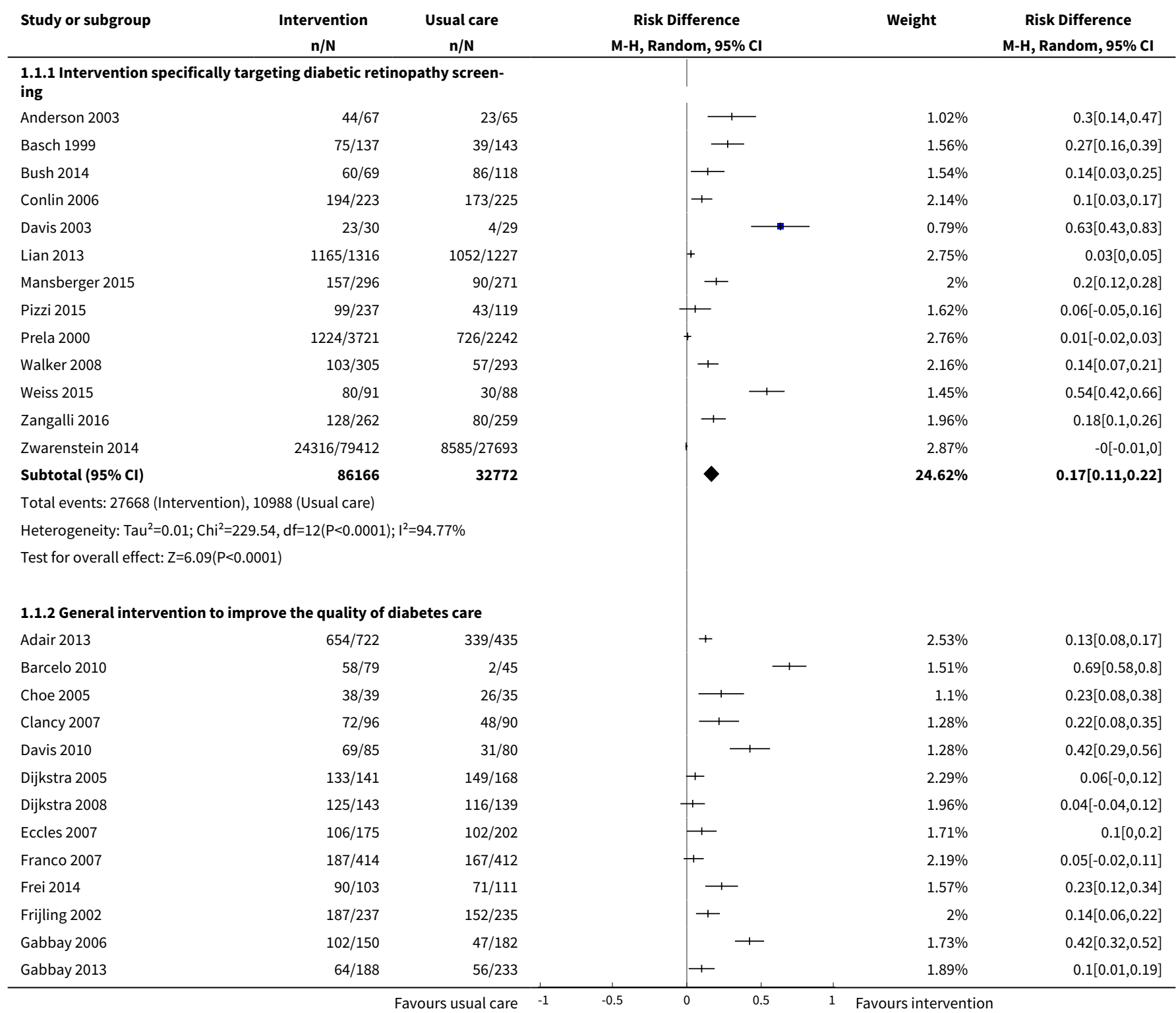




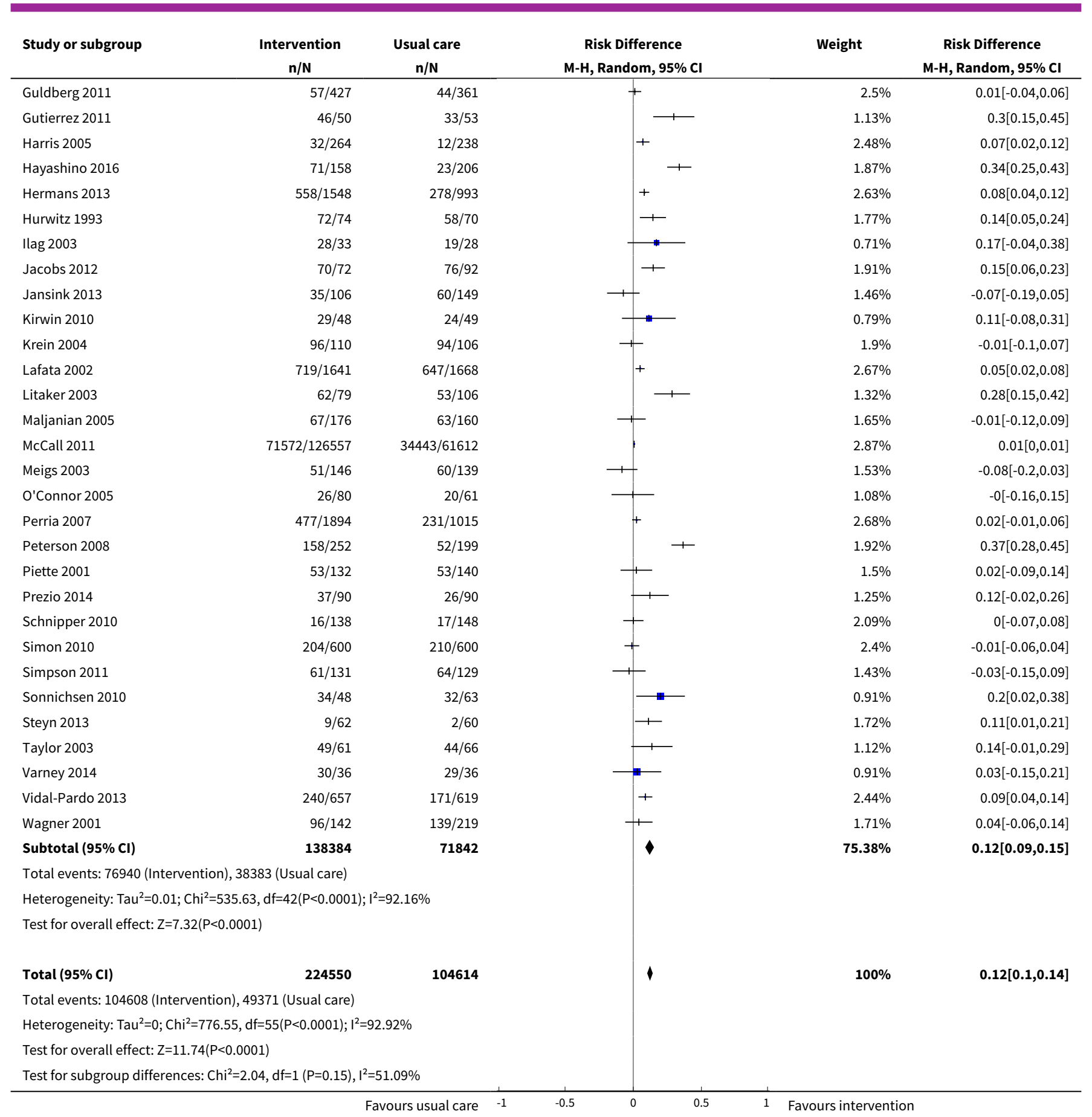

\section{Comparison 2. Stepped quality improvement intervention compared to intervention alone}

\begin{tabular}{lllll}
\hline Outcome or subgroup title & No. of studies & $\begin{array}{l}\text { No. of partici- } \\
\text { pants }\end{array}$ & Statistical method & Effect size \\
\hline $\begin{array}{l}1 \text { Proportion of participants attending } \\
\text { screening }\end{array}$ & 10 & 23715 & $\begin{array}{l}\text { Risk Difference (M-H, Ran- } \\
\text { dom, 95\% Cl) }\end{array}$ & $0.05[0.02,0.09]$ \\
\hline
\end{tabular}




\begin{tabular}{lllll}
\hline Outcome or subgroup title & No. of studies & $\begin{array}{l}\text { No. of partici- } \\
\text { pants }\end{array}$ & Statistical method & Effect size \\
\hline $\begin{array}{l}1.1 \text { Intervention specifically targeting dia- } \\
\text { betic retinopathy screening }\end{array}$ & 3 & 19698 & $\begin{array}{l}\text { Risk Difference }(\mathrm{M}-\mathrm{H}, \text { Ran- } \\
\text { dom, 95\% Cl) }\end{array}$ & $0.04[-0.11,0.19]$ \\
\hline $\begin{array}{l}1.2 \text { General intervention to improve the } \\
\text { quality of diabetes care }\end{array}$ & 7 & 4017 & $\begin{array}{l}\text { Risk Difference (M-H, Ran- } \\
\text { dom, 95\% Cl) }\end{array}$ & $0.06[0.02,0.11]$ \\
\hline
\end{tabular}

Analysis 2.1. Comparison 2 Stepped quality improvement intervention compared to intervention alone, Outcome 1 Proportion of participants attending screening.

\begin{tabular}{|c|c|c|c|c|c|}
\hline Study or subgroup & $\begin{array}{c}\text { Stepped in- } \\
\text { tervention } \\
\mathbf{n} / \mathbf{N}\end{array}$ & Control & $\begin{array}{c}\text { Risk Difference } \\
\text { M-H, Random, } 95 \% \mathrm{Cl}\end{array}$ & Weight & $\begin{array}{c}\text { Risk Difference } \\
\text { M-H, Random, } 95 \% \mathrm{CI}\end{array}$ \\
\hline \multicolumn{6}{|c|}{$\begin{array}{l}\text { 2.1.1 Intervention specifically targeting diabetic retinopathy screen- } \\
\text { ing }\end{array}$} \\
\hline Ellish 2011 & $15 / 39$ & $17 / 33$ & \begin{tabular}{l|l}
$1+$ \\
+
\end{tabular} & $2.18 \%$ & $-0.13[-0.36,0.1]$ \\
\hline Halbert 1999 & $3666 / 9909$ & $3403 / 9614$ & 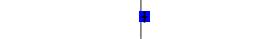 & $24.74 \%$ & $0.02[0,0.03]$ \\
\hline Rosenkranz 1996 & $49 / 66$ & $19 / 37$ & $\longrightarrow$ & $2.97 \%$ & $0.23[0.04,0.42]$ \\
\hline Subtotal $(95 \% \mathrm{Cl})$ & 10014 & 9684 & & $29.89 \%$ & $0.04[-0.11,0.19]$ \\
\hline \multicolumn{6}{|c|}{ Total events: 3730 (Stepped intervention), 3439 (Control) } \\
\hline \multicolumn{6}{|c|}{ Heterogeneity: $\mathrm{Tau}^{2}=0.01 ; \mathrm{Chi}^{2}=6.27, \mathrm{df}=2(\mathrm{P}=0.04) ; \mathrm{I}^{2}=68.08 \%$} \\
\hline \multicolumn{6}{|c|}{ Test for overall effect: $Z=0.52(P=0.6)$} \\
\hline \multicolumn{6}{|c|}{ 2.1.2 General intervention to improve the quality of diabetes care } \\
\hline Dickinson 2014 & $53 / 253$ & $20 / 162$ & $\rightarrow$ & $12.54 \%$ & $0.09[0.01,0.16]$ \\
\hline Herrin 2006 & $40 / 227$ & $10 / 97$ & $\longrightarrow$ & $11.36 \%$ & $0.07[-0.01,0.15]$ \\
\hline McClellan 2003 & $450 / 1142$ & $424 / 1072$ & + & $19.13 \%$ & $-0[-0.04,0.04]$ \\
\hline McDermott 2001 & $74 / 124$ & $80 / 174$ & $\rightarrow$ & $7 \%$ & $0.14[0.02,0.25]$ \\
\hline Ward 1996 & $96 / 231$ & $39 / 124$ & 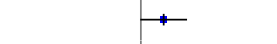 & $8 \%$ & $0.1[-0,0.2]$ \\
\hline Welch 2011 & $19 / 21$ & $14 / 18$ & $-1,1$ & $2.17 \%$ & $0.13[-0.1,0.36]$ \\
\hline Subtotal $(95 \% \mathrm{CI})$ & 2184 & 1833 & 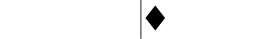 & $70.11 \%$ & $0.06[0.02,0.11]$ \\
\hline \multicolumn{6}{|c|}{ Total events: 876 (Stepped intervention), 722 (Control) } \\
\hline \multicolumn{6}{|c|}{ Heterogeneity: $\mathrm{Tau}^{2}=0 ; \mathrm{Chi}^{2}=11.13, \mathrm{df}=6(\mathrm{P}=0.08) ; \mathrm{I}^{2}=46.11 \%$} \\
\hline \multicolumn{6}{|c|}{ Test for overall effect: $Z=2.87(P=0)$} \\
\hline Total $(95 \% \mathrm{Cl})$ & 12198 & 11517 & $\bullet$ & $100 \%$ & $0.05[0.02,0.09]$ \\
\hline \multicolumn{6}{|c|}{ Total events: 4606 (Stepped intervention), 4161 (Control) } \\
\hline \multicolumn{6}{|c|}{ Heterogeneity: $\mathrm{Tau}^{2}=0 ; \mathrm{Chi}^{2}=20.32, \mathrm{df}=9(\mathrm{P}=0.02) ; \mathrm{I}^{2}=55.71 \%$} \\
\hline Test for subgroup diff & $8, d f=1(P=0.77)$ & & & & \\
\hline
\end{tabular}




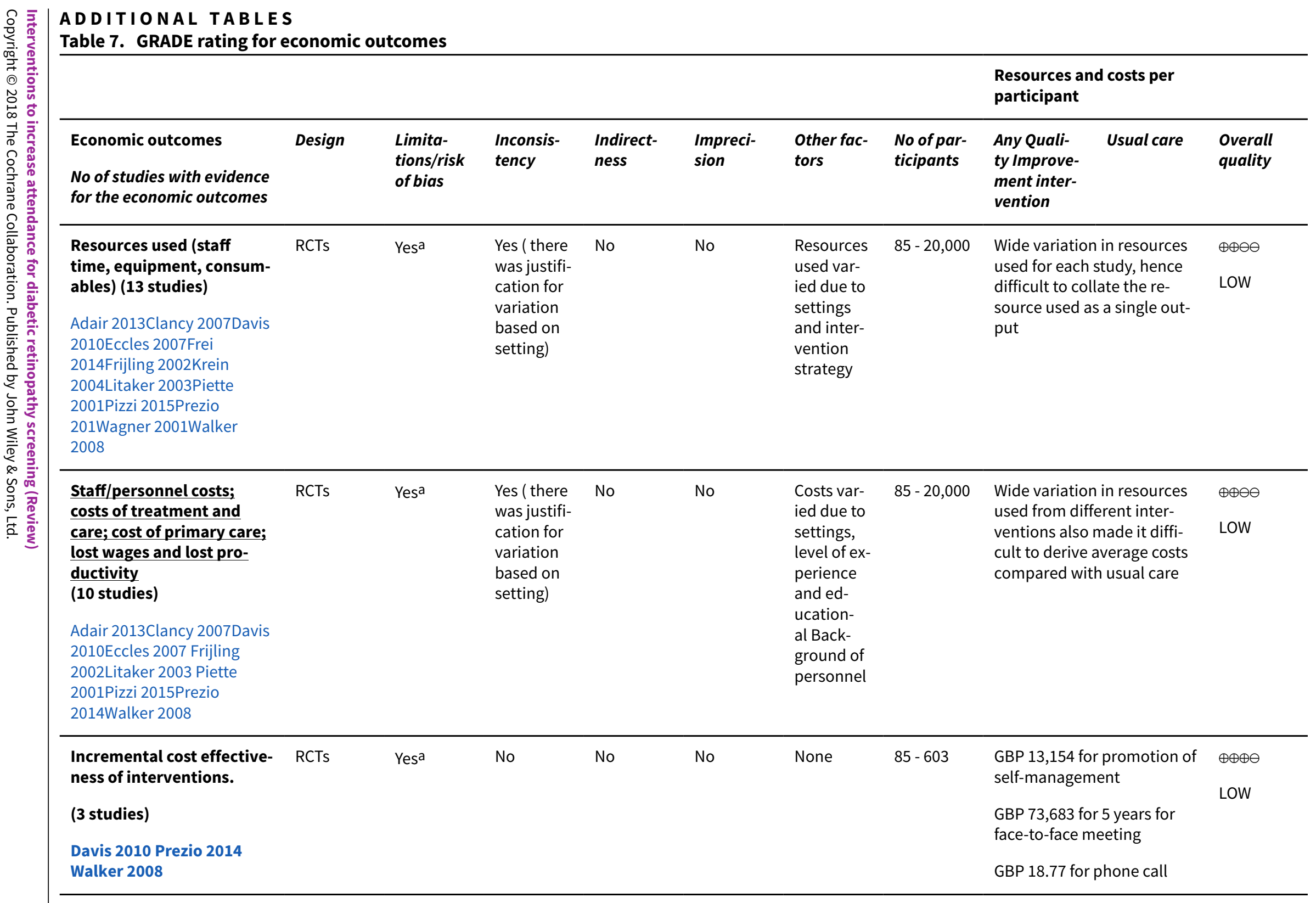


Table 1. Illustrative quotations for BCTs used in the included studies

\begin{tabular}{l} 
Behaviour change technique (BCT) and abbreviated definitions \\
\hline Goals and planning \\
Goal setting (behaviour) \\
Set or agree a goal defined in terms of the behaviour to be achieved \\
e.g. Set targets for how often patients should attend DRS, or general di- \\
abetes self-management, such as frequency of blood glucose testing, \\
amount of carbohydrates to consume at each meal
\end{tabular}

\section{Illustrative quotation}

\begin{abstract}
"Practice nurses planned independent consultations with patients. The monitoring tool guided them through the consultations, and provided the opportunity to help the patient in selecting appropriate, concrete, behavioural goals .... The monitoring tool addressed clinical parameters (e.g., $\mathrm{HbA}_{1 \mathrm{C}}$, BP and LDL cholesterol levels), examinations (e.g. food control, neurological tests, and eye examinations), adherence to prescribed drugs, selfcare goals, and other recommendations" (Frei 2014 p 1040-1)
\end{abstract}

\section{Problem solving}

Analyse, or prompt the person to analyse, factors influencing the behaviour and generate or select strategies that include overcoming barriers and/or increasing facilitators

e.g. Support patients to identify reasons for wanting or not wanting to attend DRS, and helping them select potential strategies for overcoming these barriers to screening attendance

\section{Goal setting (outcome)}

Set or agree a goal defined in terms of a positive outcome of wanted behaviour

e.g. Agree with the patient target $\mathrm{HbA1c}$, blood pressure, or cholesterol level, or target range for blood glucose

\section{Action planning}

Prompt detailed planning of performance of the behaviour

e.g. Support the patient to develop a plan for how often they will attend DRS, where the DRS will occur, and how they will get to their appointment
"Using a semi structured protocol, the health educator (C.J. H.) offered one-on-one, interactive education and counselling. Having established rapport, she worked to identify and understand each subject's reasons for and /or barriers to having a dilated retinal examination. Focused problem-solving then guided the subject toward making an informed choice about receiving an ophthalmic examination." (Basch 1999, p 1879)
"During the case management sessions, patients and providers set management goals that were reasonable to achieve." (Barcelo 2010, p 147) betes mellitus, behavioural therapy, and the health be-

\section{Review behaviour goals}

Review behaviour goal(s) jointly with the person and consider modifying goal(s) or behaviour change strategy in light of achievement

e.g. During scheduled diabetic review consultations, discuss with patients how they are progressing with their agreed self-management behavioural goals (e.g. frequency of blood glucose testing, attendance for DRS). Where patients are not meeting agreed goals, either discuss how to adjust goals if needed to increase feasibility, or engage in problem-solving to overcome any barriers to goal attainment
"Behavioural activation for diabetic retinopathy prevention combined the principles of education about dialief model to assist participants in identifying barriers to obtaining dilated fundus examinations, problems-solving solutions to surmounting barriers, formulating action plans to facilitate dilated retinal examinations, and gauging the success of action plans." (Weiss 2015, p 1007)

"Care managers were trained to use a patient-centred self-management approach that included review of the medical care needs and self-care goals that the patient identified and brainstorming additional strategies that patients could use to overcome barriers to their goals." (Glasgow 2005, p 35)

\section{Discrepancy between current and goal}

"Physicians in the IG [intervention group] received a monthly report of their care quality with the top $10 \%$ quality of diabetes care score for all physicians being the achievable benchmark."(Hayashino 2016, p 1) 
Table 1. Illustrative quotations for BCTs used in the included studies (Continued)

Draw attention to discrepancies between a person's current behaviour and the person's previously set outcome goals, behaviour goals or action plans

e.g. Provide feedback to healthcare professionals on the proportion of patients who have received DRS in the previous 12 months, and compare this against a gold standard for clinical practice based on clinical guidelines

\section{Review outcome goal(s)}

Review outcome goal(s) jointly with the person and consider modifying goal(s) in light of achievement

e.g. Review or alter target blood glucose levels towards a more feasible/achievable intermediate target
"The telephone call was structured to first review the patient's goals, followed by medication use, symptoms, glucose monitoring, blood pressure monitoring and selfmanagement /care activities" (Taylor 2003, p 1059)

\section{Behavioural contract}

Create a written specification of the behaviour to be performed, agreed by the person, and witnesses by another

Care guides asked patients to sign a contract (which was scanned into the HHR) agreeing to work toward their disease-specific goals. (Adair 2013, p 176)

e.g. Ask the person with diabetes to sign a contract in their self-management plan or diary, undertaking to attend DRS once

\section{Commitment}

Ask the person to affirm or reaffirm statement indicating commitment to

"The initial goal was toelicit a verbal commitment to schedule an eye examination." (Basch 1999, p 1879) change the behaviour

e.g. Ask the person with diabetes to verbally affirm or reaffirm that they are committed to attending DRS at the agreed frequency and location

\section{Feedback and monitoring}

\section{Monitoring of behaviour by others without feedback}

Observe or record behaviour with the person's knowledge as part of a behaviour change strategy

e.g. Record the proportion of patients who attend for a DRS exam as part of clinical audit, but the results are not fed back to the healthcare professionals whose practice has been audited

\section{Feedback on behaviour}

Monitor and provide information or evaluative feedback on performance of the behaviour (e.g. form, frequency, duration, intensity)

e.g. Provide a feedback report to healthcare professionals, stating the proportion of their patients who have attended a DRS exam, had their blood pressure taken, and had a foot examination

\begin{abstract}
"Foot examinations, blood pressure, and eye examinations were recorded on the reminder by clinic staff, collected after the patient visit and entered manually." (Peterson 2008, p 2239)
\end{abstract}

\section{Self-monitoring of behaviour}

Establish a method for the person to monitor and record their behaviour(s) as part of a behaviour change strategy

e.g. A person with diabetes maintains a self-management diary in which they record their daily food intake and exercise, and tick off a checklist when they have attended their annual DRS exam
"In addition, diabetic members who did not have a record of a diabetic retinopathy exam received educational materials and a report of their current DRE status directly from the HMO 2 weeks later." (Halbert 1999, p 753)
"We prepared feedback sheets for adherence to these eight indicators using data from thephysicians' selfreport forms, as the physicians monitored and promoted these indicators to improve adherence." (Hayashino 2015, p 601) 
Table 1. Illustrative quotations for BCTs used in the included studies (Continued)

\section{Self-monitoring of outcomes of behaviour}

Establish a method for the person to monitor and record the outcome(s) of their behaviour as part of a behaviour change strategy

e.g. A person with diabetes records in their self-management diary the results of their latest $\mathrm{HbA1C}$ result and DRS exam

\section{Monitoring of outcomes of behaviour by others without feedback \\ Observe or record outcomes of behaviour with the person's knowledge as part of a behaviour change strategy \\ e.g. A person attends a DRS exam, but is not provided with the results of the examination}

"In general, case managers were directed to encourage patient self-management, including diet and exercise, provide reminders for recommended screening/tests,help with appointment scheduling;monitoring home glucose and blood pressure levels..." (Krein 2004, p 734)

"The nurse case manager used behavioural goals setting, established individualized care plan, provide patient self-management education and surveillance of patients...ordered protocol-driven laboratory tests, tracked the outcomes using the computerized data registry..." (Gabbay 2006, p 30)

\section{Feedback on outcomes of behaviour}

Monitor and provide feedback on the outcome of performance of the behaviour

e.g. Informing the person with diabetes of the results of DRS exam [i.e. presence/absence of retinopathy] "...all persons who attended the screening clinics received a dilated eye exam by a volunteer community-based ophthalmologist. The eye exam included visual acuity, intraocular pressure, and a fundus examination through a dilated pupil...immediately after receiving the dilated eye exam, the patient was told the results by the examination ophthalmologist." (Anderson 2003, p 41)

"... immediately after receiving the dilated eye exam, the patient was told the results by the examination ophthalmologist." (Anderson 2003, p 41)

Provide feedback about the body (e.g. physiological or biochemical state) using an external monitoring device as part of a behaviour change strategy

\section{Social Support}

\section{Social Support (unspecified)}

Advise on, arrange or provide social support (e.g. from friends, relatives, colleagues, 'buddies' or staff) or non-contingent praise or reward for performance of the behaviour. In includes encouragement and counselling

e.g. Provide general encouragement or reassurance to a person with diabetes to attend their DRS appointment

\section{Social Support (practical)}

Advise on, arrange, or provide practical help (e.g. from friends, relatives, colleagues, 'buddies' or staff) for performance of the behaviour

e.g. Provide practical help for a patient with diabetes to attend DRS. This can include, for example: arranging a referral to DRS, arranging or providing transport to the clinic
"Overall, the intervention included ....and self-management support (provided by the practice nurse)." (Frei 2014, p 1041)

\section{Shaping knowledge}

\section{Instruction on how to perform behaviour}

Advise or agree on how to perform the behaviour (includes 'skills training')

e.g. Provide advice to a person with diabetes on how often guidelines recommend attending DRS, where they can obtain a DRS, and how to schedule an eye exam
"Referrals were facilitated to other clinicians when indicated, including ophthalmology, podiatry, nutrition and primary care for follow-up of acute or other chronic issues or when requested by patients." (Jacobs 2012, p 616)
"A direct mail reminder was sent to patients to reinforce the importance of annual eye exams and included the following text:

If you don't have an eye doctor, ask you regular doctor to refer you to one." (Prela 2000, p 258) 
Table 1. Illustrative quotations for BCTs used in the included studies (Continued)

\section{Natural consequences}

\section{Information about health consequences}

Provide information (e.g. written, verbal, visual) about health consequences of performing the behaviour

e.g. Provide advice to the person with diabetes, on the negative health consequences of retinopathy, and the benefits of early detection
"A tailored telephone intervention was delivered by bilingual interventionists and included: Risk communications, such as the frequency lack of symptoms of retinopathy and that early treatment for retinopathy decreases the risk of blindness, were included." (Walker 2008, p 187)

\section{Salience of consequences}

Use methods specifically designed to emphasise the consequences of performing the behaviour with the aim of making them more memorable

e.g. Give a person with diabetes a leaflet containing testimonials from other persons with diabetes who suffer from retinopathy to emphasise the benefits of attending DRS and early detection

\section{Information about social \& environmental consequences}

Provide information (e.g. written, verbal, visual) about social and environmental consequences of performing the behaviour

e.g. Provide information on the costs of having a DRS exam
"The videotape used emotional appeals through storytelling to increase motivation to have a yearly dilated retinal examination." (Basch 1999, p 1879)

\section{Information about emotional consequences}

Provide information (e.g. written, verbal, visual) about emotional consequences of performing the behaviour

e.g. Provide a leaflet recognising the potential negative effects on emotional and mental health of managing a chronic illness, such as diabetes
"A take-home reminder (aimed at patients, to remind them to make an appointment for an eye exam), to be given to patients by their Family Practitioner, included the following text:

OKIP covers annual eye checks for patients with diabetes so you will not have to pay" (Zwarenstein 2014, p 90)

"Group visit content, though patient-guided, was physician-directed to cover educational topics...and the emotional aspects of diabetes." (Clancy 2007, p 621)

\section{Comparison of behaviour}

\section{Demonstration of the behaviour}

Provide an observable sample of the performance of the behaviour, directly in person or indirectly (e.g. by film, picture, for the person to aspire to or imitate)

e.g. Play a video demonstrating the DRS procedure

\section{Social comparison}

Draw attention to others' performance to allow comparison with the person's own performance

e.g. Provide healthcare professionals with feedback on the proportion of their patients who have had a DRS exam, and benchmark this in comparison to other hospitals or healthcare professionals
"The newsletter consisted of six sections, including a testimonial designed to model eye examination behaviour" (Ellish 2011, p 1593)

\section{Information about others' approval}

Provide information about what other people think about their behaviour. The information clarifies whether others will like, approve or disapprove of what the person is doing or will do

\begin{abstract}
"The system presented register data on their' Type 2 diabetes population, giving them the option either to use the data during individual diabetes consultations or to gain an overview of the quality of their diabetes care and compare it with the corresponding quality in their colleagues' practices." (Guldberg 2011, p 326)
\end{abstract}

"One of the message in the targeted newsletter read:

Even though you've been thinking about getting a dilated eye exam, we hope you'll make the call now"(Ellish 2011, Additional information provided by the author) 
Table 1. Illustrative quotations for BCTs used in the included studies (Continued)

e.g. Tell the person with diabetes that their family members would likely be keen for them to attend their DRS appointment

\section{Associations}

\section{Prompts/Cues}

Introduce or define environmental or social stimulus with the purpose of prompting or cueing the behaviour

e.g. Phone the person with diabetes to remind them of their upcoming DRS appointment

\section{Reduce prompts/cues}

Withdraw gradually prompts to perform the behaviour

e.g. Decrease the frequency with which a person with diabetes is sent a reminder of their DRS attendance (i.e. from weekly, to fornightly, to monthly, to quarterly reminders)
"For those who made an appointment, a reminder letter was mailed 3 weeks prior to the scheduled appointment. Additionally, there was an automated reminder call the day before the scheduled appointment" (Pizzi 2015, p 255)

\section{Repetition and substitution}

\section{Behavioural practice/rehearsal}

Prompt practice or rehearsal of the performance of the behaviour one or more times in a context or at a time when the performance may not be necessary, in order to increase habit and skill

e.g. Provide an opportunity for trainee healthcare professionals to practise delivering a DRS exam to an actor role-playing a patient with diabetes
"During a 2-day training session, case managers received instruction on collaborative goal setting, with case examples and role-playing used to familiarize them with the treatment algorithms"( Krein 2004, p 734)

\section{Graded tasks}

Set easy-to-perform tasks, making them increasingly difficult, but achievable, until the behaviour is performed

e.g. Initially allocate a healthcare professional responsibility for one component of DRS exam and progressively increase their responsibility
"Recommendations for regular telephone follow-ups for diabetes patients, which will be monthly in the $1^{\text {st }}$ half year and then will probably decrease" (Jansink 2013 (coded from protocol 2009)

\section{Comparison of outcomes}

\section{Credible source}

Present verbal or visual communication from a credible source in favour of or against the behaviour

e.g. Include the logos for national health institutes, or cite published clinical guidelines, to endorse information provided in leaflets regarding DRS
"Theoretically, this form of facilitation should be necessary for only a relatively short period of time, with the practice improvement team progressively assuming responsibility for the ongoing improvement efforts after the initial facilitation." (Dickinson 2014, p 10)

\section{Reward and threat}

\section{Material incentive (behaviour)}

Inform that money, vouchers or other valued objects will be delivered if and only if there has been effort and/or progress in performing the behaviour

e.g. Advise the person with diabetes that they will receive a shopping voucher if they attend their upcoming DRS appointment
"Participants in the print-intervention group received a mailing of a colourful, 14-page booklet on preventing diabetes eye problems called Keep Your Eyes Healthy, in English or Spanish, developed b y the National Institutes of Health." (Walker 2008, p 187)
"The automated system offered a live telephone call back to assist in scheduling test and alsooffered to send participants the following items: 1 ) a voucher that would allow the provider to waive the co-payment for a dilated eye examination..." (Simon 2010, p 1452) 


\section{Table 1. Illustrative quotations for BCTs used in the included studies (Continued)}

\section{Social reward \\ Arrange verbal or non-verbal reward if and only if there has been effort and/or progress in performing the behaviour \\ e.g. Verbally praise the person with diabetes if they attend their DRS ap- pointment}

"When a subject reported having a dilated retinal examination a congratulatory letter was sent." (Basch 1999, p 1879)

\section{Non-specific reward}

Inform that a reward will be delivered if and only if there has been effort "CME credits were given to the participating physicians in the workshops" (Vidal-Pardo 2013, p 752)

e.g. Inform the healthcare professional that they will be rewarded for conducting a DRS exam with a target proportion of their patients

\section{Antecedents}

\section{Restructuring the physical environment}

Change or advise to change the physical environment in order to facilitate performance of the wanted behaviour or create barriers to the unwanted behaviour

e.g. Introduce mobile DRS vans in geographically remote areas to increase access to screening facilities

\section{Restructuring the social environment}

Change or advise to change the social environment in order to facilitate performance of the wanted behaviour or create barriers to the unwanted behaviour

e.g. Change a healthcare team and team working, such as introducing a new specialist diabetes nurse role responsible for monitoring screening rates and phoning people with diabetes to remind them to attend their DRS appointment

\section{Adding objects to the environment}

Add objects to the environment in order to facilitate performance of the behaviour

e.g. Introduce new computerised software to a general practice to help monitor and remind healthcare professionals as to which patients need to be prompted to attend DRS
"Care guide workstations were located in the clinic waiting rooms, to facilitate face-to-face interactions with patients, providers, and nurses." (Adair 2013, p 177)

\section{Scheduled consequences}

\section{Behaviour cost}

Arrange for withdrawal of something valued if and only if an unwanted behaviour is performed

e.g. Charging people with diabetes a fee for failing to attend a DRS exam
"Three multi-lingual Link Workers already employed by Coventry Primary Care Trust (PCT) were trained in diabetes management and care and assigned to work with specific intervention GP surgeries" (Bush 2014, p 295)
"In addition 4500 diabetes passports were made available at the four hospitals..." (Dijkstra 2005, p 128)

\section{Self-belief}

Verbal persuasion about capability

"We were interested to find out whether a small copayment would be an important deterrent to the uptake of screening for diabetic retinopathy (DR)...We conducted a randomized trial in which one group was charged a small fee for DR screening and the other was provided with free access." (Lian 2013, p 1247) 
Table 1. Illustrative quotations for BCTs used in the included studies (Continued)

Tell the person that they can successfully perform the wanted behaviour, charge of your health, not only for today, but also for the arguing against self-doubts and asserting that they can and will succeed years to come" (Lafata 2002, p 523)

e.g. Encourage or reassure the patient to attend a DRS exam, providing information as needed to address any concerns or self-doubts they may have about attending for a DRS exam

\section{Focus on past success}

Advise to think about or list previous successes in performing the behaviour (or parts of it)

e.g. Help the person with diabetes to remember the last time they attended a DRS exam, and use this as an opportunity to reassure them of the benefits of attending
A comprehensive programme that integrated lifestyle: counselling based on motivational interviewing principles was integrated into structured diabetes care.

[In description of motivational interviewing] "Self-efficacy can be strengthened by affirming past success (i.e. reinforcement)..." (Jansink 2013 , additional information from protocol)

DRS: diabetic retinopathy screening

Table 2. Summary of reported costs and resources to deliver interventions

\begin{tabular}{|c|c|c|c|c|}
\hline QI Component & Study & DRS or GQI & Estimated costs of resources used & Resources used \\
\hline \multirow[t]{2}{*}{$\begin{array}{l}\text { Promotion of } \\
\text { self-manage- } \\
\text { ment }\end{array}$} & $\begin{array}{l}\text { Davis } 2010 \\
\mathrm{~N}=85 \text { partici- } \\
\text { pants }\end{array}$ & GQI & $\begin{array}{l}\text { Staff cost per person = GBP } 625.25 ; \\
\text { costs of the other resources used = GBP } \\
476.35 \text { over } 12 \text { months } \\
\text { Direct cost per person = GBP } 1101\end{array}$ & $\begin{array}{l}13 \times 15 \text {-minute sessions ( } 3 \\
\text { individuals and } 10 \text { group } \\
\text { session) with nurses and } 4 \\
\text { hours with health educator } \\
\text { per person }\end{array}$ \\
\hline & $\begin{array}{l}\text { Wagner } 2001 \\
\mathrm{~N}=14 \text { clinics, } \\
278 \text { participants }\end{array}$ & GQI & Not reported & $\begin{array}{l}\text { 1-hour group session with } \\
\text { relevant health professional } \\
\text { every } 3 \text { - } 6 \text { months }\end{array}$ \\
\hline \multirow[t]{3}{*}{ Team changes } & $\begin{array}{l}\text { Frei } 2014 \\
\mathrm{~N}=15 \text { practices, } \\
164 \text { participants }\end{array}$ & GQI & Not reported & $\begin{array}{l}\text { 6-day training for nurses, } 2 \mathrm{x} \\
\text { 4-hour workshops for physi- } \\
\text { cians and nurses }\end{array}$ \\
\hline & $\begin{array}{l}\text { Wagner } 2001 \\
N=14 \text { clinics, } \\
278 \text { participants }\end{array}$ & GQI & Not reported & $\begin{array}{l}\text { 1-hour group session with } \\
\text { relevant health professional } \\
\text { every } 3 \text { - } 6 \text { months }\end{array}$ \\
\hline & $\begin{array}{l}\text { Litaker } 2003 \\
\mathrm{~N}=79 \text { partici- } \\
\text { pants }\end{array}$ & GQI & $\begin{array}{l}\text { Mean personnel costs for the interven- } \\
\text { tion per month per patient = GBP } 130.15 \\
\text { Total additional personnel costs = GBP } \\
10281.97\end{array}$ & $\begin{array}{l}\text { An average of } 180 \text { minutes } \\
\text { with participants }\end{array}$ \\
\hline $\begin{array}{l}\text { Case manage- } \\
\text { ment }\end{array}$ & $\begin{array}{l}\text { Krein } 2004 \\
\mathrm{~N}=123 \text { partici- } \\
\text { pants }\end{array}$ & GQI & Not reported & $\begin{array}{l}2 \text { days training for case } \\
\text { managers, } 20 \text { hours/week } \\
\text { time spent with partici- } \\
\text { pants. Quarterly profiling } \\
\text { and subsequently every } 6 \\
\text { months }\end{array}$ \\
\hline $\begin{array}{l}\text { Patient educa- } \\
\text { tion }\end{array}$ & Prezio 2014 & GQI & Physician cost $=$ GBP 48.76/hour & $\begin{array}{l}7 \text { sessions per participants, } \\
1 \text { hour physician supervi- } \\
\text { sion for health workers }\end{array}$ \\
\hline
\end{tabular}


Table 2. Summary of reported costs and resources to deliver interventions (Continued) $\mathrm{N}=90$ particiCommunity health worker $=$ GBP 12.91/ pants hour

Cost of intervention over 20 years $=\mathrm{GBP}$ 3646.10 per patient

Pizzi 2015
$\mathrm{~N}=117$ partici-
pants for mailed
intervention, 120
for telephone in-
tervention

DRS

Staff time for 120 participants $=\mathrm{GBP}$ 501.13 for telephone over 1 month

Staff time for 117 participants $=$ GBP 173.17 for mailed intervention over 1 month

GBP $85.24 /$ hour for the physician, GBP 29.32/hr for health services manager, GBP 16.72/hour for medical assistant

Cost of materials for telephone $=\mathrm{GBP}$ 30.25 , cost of materials for mailed intervention

Total cost of intervention = GBP 577.64 for 120 participants in telephone group, GBP 335.48 for 117 participants in mailed group over a month

Total cost when appointment is made and kept per participant;

Telephone intervention $=$ GBP 9.47

Mailed intervention $=$ GBP 8.83

\begin{tabular}{|c|c|c|c|}
\hline $\begin{array}{l}\text { Adair } 2013 \\
\mathrm{~N}=930 \text { partici- } \\
\text { pants }\end{array}$ & GQI & $\begin{array}{l}\text { Care guide cost for } 120 \text { participants = } \\
\text { GBP } 375,917 \text { at the rate of GBP } 11.77 / \\
\text { hour over a year } \\
2 \text { supervisory nurses = GBP } 85,847.24 \\
\text { Training cost = GBP } 2228.99 \\
\text { modular furniture and equipment for } 12 \\
\text { stations = GBP } 79,422.81 \\
\text { Total cost = GBP } 463,993 \\
\text { Total cost of intervention per partici- } \\
\text { pant = GBP } 326\end{array}$ & $\begin{array}{l}12 \text { care guides, } 2 \text { weeks } \\
\text { training, } 2 \text { supervisory nurs- } \\
\text { es, } 5 \text { visits on average to } \\
\text { clinics, } 4 \text { contacts with par- } \\
\text { ticipants, furniture and } \\
\text { modular equipment }\end{array}$ \\
\hline $\begin{array}{l}\text { McCall } 2011 \\
\text { N = approximate- } \\
\text { ly } 20,000 \text { partici- } \\
\text { pants }\end{array}$ & GQI & Not reported & Not reported \\
\hline $\begin{array}{l}\text { Clancy } 2007 \\
\text { N=96 partici- } \\
\text { pants }\end{array}$ & GQI & $\begin{array}{l}\text { Deposit fee for group visit = GBP 13.4/ } \\
\text { visit, for } 12 \text { visits = GBP } 160.60\end{array}$ & $\begin{array}{l}\text { Monthly meeting for a year } \\
\text { for } 2 \text { hours which includes } 1 \\
\text { primary care internal medi- } \\
\text { cine physician, } 1 \text { registered } \\
\text { nurse per visit } \\
\text { Training for physicians and } \\
\text { nurses }\end{array}$ \\
\hline
\end{tabular}

1-hour supervision for every 20-hour intervention delivered

$2 \times 1$-hour meetings with services manage and ophthalmologist medical assistants, health nurses 
Table 2. Summary of reported costs and resources to deliver interventions (Continued)

3- hour training for clinic staff

12 group visits for 1 year

\begin{tabular}{lll}
\hline $\begin{array}{l}\text { Schechter 2008 } \\
\text { (Walker 2008) }\end{array}$ & DRS & $\begin{array}{l}\text { Costs of health educator }=\text { GBP } \\
14,890.83\end{array}$
\end{tabular}

$\mathrm{N}=305$ partic-

Training and supervision = GBP 2756.44

ipants for tele-

phone interven-

tion, 298 for print

intervention
Average of 3.2 calls for about 20 minutes +5 minutes call preparation per participant over 6 months

20 hours training, 1 hour supervision by diabetes nurse educator, telephone calls
Telephone charges $=$ GBP 679.67 for 305 participants

Costs of printing and mailing $=\mathrm{GBP}$ 465.99 for 298 participants

\begin{tabular}{|c|c|c|c|c|}
\hline $\begin{array}{l}\text { Electronic pa- } \\
\text { tient register }\end{array}$ & $\begin{array}{l}\text { Eccles } 2007 \\
N=30 \text { pract }\end{array}$ & GQI & $\begin{array}{l}\text { Cost of developing the guidelines }=\text { GBP } \\
10,208\end{array}$ & $\begin{array}{l}\text { Cost of guidelines and soft- } \\
\text { ware development. Average } \\
\text { of } 2 \text { follow-up contacts }\end{array}$ \\
\hline
\end{tabular}

12519.36

Cost of educational activities $=\mathrm{GBP}$

2148.11

Additional cost of running the system = GBP 9964.46

Annual cost per participant $=$ GBP 68.21

\begin{tabular}{|c|c|c|c|c|}
\hline & $\begin{array}{l}\text { Schechter } 2008 \\
\text { (Walker 2008) } \\
\mathrm{N}=305 \text { partic- } \\
\text { ipants for tele- } \\
\text { phone interven- } \\
\text { tion, } 298 \text { for print } \\
\text { intervention }\end{array}$ & DRS & $\begin{array}{l}\text { Costs of health educator = GBP } \\
14,890.83 \\
\text { Training and supervision = GBP } 2756.44 \\
\text { Telephone charges = GBP } 679.67 \text { for } 305 \\
\text { participants } \\
\text { Costs of printing and mailing = GBP } \\
465.99 \text { for } 298 \text { participants }\end{array}$ & $\begin{array}{l}\text { Average of } 3.2 \text { calls for } \\
\text { about } 20 \text { minutes }+5 \text { min- } \\
\text { utes call preparation per } \\
\text { participant over } 6 \text { months } \\
20 \text { hours training, } 1 \text { hour su- } \\
\text { pervision by diabetes nurse } \\
\text { educator, telephone calls }\end{array}$ \\
\hline & $\begin{array}{l}\text { Pizzi } 2015 \\
\mathrm{~N}=117 \text { partici- } \\
\text { pants for mailed } \\
\text { intervention, } 120 \\
\text { for telephone in- } \\
\text { tervention }\end{array}$ & DRS & $\begin{array}{l}\text { Staff time for } 120 \text { participants = GBP } \\
501.13 \text { for telephone over } 1 \text { month } \\
\text { Staff time for } 117 \text { participants = GBP } \\
173.17 \text { for mailed intervention over } 1 \\
\text { month } \\
\text { GBP } 85.24 \text { /hour for the physician, GBP } \\
29.32 / \text { hour for health services manager, } \\
\text { GBP } 16.72 \text { /hour for medical assistant } \\
\text { Cost of materials for telephone = GBP } \\
30.25 \text {, cost of materials for mailed inter- } \\
\text { vention } \\
\text { Total cost of intervention = GBP } 577.64 \\
\text { for } 120 \text { participants in telephone group, } \\
\text { GBP } 335.48 \text { for } 117 \text { participants in } \\
\text { mailed group over a month }\end{array}$ & $\begin{array}{l}1 \text { hour supervision for every } \\
20 \text {-hour intervention deliv- } \\
\text { ered } \\
2 \times 1 \text {-hour meetings with } \\
\text { medical assistants, health } \\
\text { services manager and oph- } \\
\text { thalmologist }\end{array}$ \\
\hline $\begin{array}{l}\text { Audit and feed- } \\
\text { back }\end{array}$ & Frijling 2002 & GQI & $\begin{array}{l}\text { Cost of clinical decision-making per } \\
\text { practice = GBP } 341.51\end{array}$ & $\begin{array}{l}80 \text { hours training for facil- } \\
\text { itator, } 15 \times 1 \text {-hour visits to }\end{array}$ \\
\hline
\end{tabular}


Table 2. Summary of reported costs and resources to deliver interventions (Continued)

$\mathrm{N}=62$ clusters,

703 participants practice clinic, 3 hours GP time for implementation of feedback

An average of 180 minutes with participants over 12 months $10,281.97$

Approximately GBP 14 - GBP 24 per year for automated calls.
13 nurses spending an average of 3.8 hours per participant, 15 automated calls quality im-

provements

Piette $2001 \quad$ GQI
$N=146$ partici-
pants

\section{GQI} pants
Total additional personnel costs $=\mathrm{GBP}$

Mean personnel costs for the intervention per month $=$ GBP 130.15

$\mathrm{N}=79$ partici pants

DRS: diabetic retinopathy screening

GQI: general quality improvement

Table 3. Summary of characteristics of included studies

\begin{tabular}{|c|c|c|c|}
\hline $\begin{array}{l}\text { Study characteris- } \\
\text { tics }\end{array}$ & $\begin{array}{l}\text { Target: diabetic retinopathy } \\
\text { screening attendance } \\
\mathrm{N}=16\end{array}$ & $\begin{array}{l}\text { Target: general quality im- } \\
\text { provement in diabetes care } \\
N=50\end{array}$ & $\begin{array}{l}\text { TOTAL } \\
N=66\end{array}$ \\
\hline \multirow[t]{7}{*}{ Study design } & Individual RCT: & Individual RCT: & Individual RCT \\
\hline & $\mathrm{n}=14(87.5 \%)$ & $n=21(42 \%)$ & $n=35(53 \%)$ \\
\hline & Cluster-RCT: & Cluster-RCT: & Cluster-RCT \\
\hline & $\mathrm{n}=2(12.5 \%)$ & $\mathrm{n}=29(58 \%)$ & $\mathrm{n}=31(47 \%)$ \\
\hline & 2 arms $n=13(81.3 \%)$ & 2 arms $n=46(92 \%)$ & 2 arms $n=59(89.4 \%)$ \\
\hline & 3 arms $n=2(12.5 \%)$ & 3 arms $n=4(8 \%)$ & 3 arms $n=6(9.1 \%)$ \\
\hline & $>3$ arms $n=1(6.3 \%)$ & & $>3$ arms $n=1(1.5 \%)$ \\
\hline \multirow[t]{7}{*}{ Location } & USA: $n=12(75 \%)$ & USA: $n=29(58 \%)$ & USA: $n=41(62.1 \%)$ \\
\hline & Canada: $\mathrm{n}=1(6.3 \%)$ & Canada: $\mathrm{n}=2(4 \%)$ & Canada: $\mathrm{n}=3(4.6 \%)$ \\
\hline & China: $\mathrm{n}=1(6.3 \%)$ & Netherlands: $\mathrm{n}=4(8 \%)$ & Netherlands: $\mathrm{n}=4(6.1 \%)$ \\
\hline & Germany: $n=1(6.3 \%)$ & Australia: $n=3(6 \%)$ & Australia: $n=3(4.6 \%)$ \\
\hline & UK: $n=1(6.3 \%)$ & UK: $n=2(4 \%)$ & UK: $n=3(4.6 \%)$ \\
\hline & Conducted between 1995 and 2013 & Other $n=10(20 \%)$ & Other: $\mathrm{n}=12(18.2 \%)$ \\
\hline & & $\begin{array}{l}\text { Conducted between } 1988 \text { and } \\
2013\end{array}$ & $\begin{array}{l}\text { Conducted between } 1988 \text { and } \\
2013\end{array}$ \\
\hline \multirow[t]{4}{*}{ Setting } & Primary care: & Primary care: & Primary care: \\
\hline & $\mathrm{n}=11(68.8 \%)$ & $\mathrm{n}=40(80 \%)$ & $\mathrm{n}=51(77.3 \%)$ \\
\hline & Outpatient clinics: & Outpatient n = $3(6 \%)$ & Outpatient clinics \\
\hline & $n=4(25 \%)$ & Unclear: $n=7(14 \%)$ & $\mathrm{n}=7(10.6 \%)$ \\
\hline
\end{tabular}


Table 3. Summary of characteristics of included studies (Continued)

Unclear: $n=1(6.3 \%)$

Unclear $\mathrm{n}=8(12.1 \%)$

\begin{tabular}{|c|c|c|c|}
\hline \multirow[t]{6}{*}{ Diabetes type } & Type 2: & Type 2: & Type 2 : \\
\hline & $n=4(25 \%)$ & $n=34(68 \%)$ & $\mathrm{n}=38(57.6 \%)$ \\
\hline & Type 1 and Type 2: & Type 1 and Type 2 & Type 1 and 2 \\
\hline & $\mathrm{n}=3(18.8 \%)$ & $n=7(14 \%)$ & $\mathrm{n}=10(15.2 \%)$ \\
\hline & Not reported: & Not reported: & Not reported \\
\hline & $\mathrm{n}=9(56.3 \%)$ & $\mathrm{n}=9(18 \%)$ & $\mathrm{n}=18(27.3 \%)$ \\
\hline \multirow{3}{*}{$\begin{array}{l}\text { Number of partici- } \\
\text { pants recruited }\end{array}$} & Individual RCT = 38,273 & Individual RCT = 198,752 & Individual RCT = 237,025 \\
\hline & $\begin{array}{l}\text { Cluster RCT = } 4135 \text { clusters, 182,513 } \\
\text { participants }\end{array}$ & $\begin{array}{l}\text { Cluster } \mathrm{RCT}=1991 \text { clusters, } \\
78,276 \text { participants }\end{array}$ & $\begin{array}{l}\text { Cluster } \mathrm{RCT}=6126 \text { clusters, } \\
260,789 \text { participants }\end{array}$ \\
\hline & Total: 220,786 participants included & $\begin{array}{l}\text { Total: } 277,028 \text { participants in- } \\
\text { cluded }\end{array}$ & $\begin{array}{l}\text { Total: } 497,814 \text { participants in- } \\
\text { cluded }\end{array}$ \\
\hline \multirow[t]{2}{*}{ Median age } & Median 60.7 yrs (range 51.1 - 72.7) & Median 60.6 yrs (range 46.8 - 74) & Median 60.7 yrs (46.8 - 74) Num- \\
\hline & 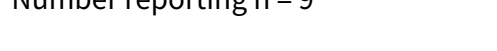 & Number reporting $n=34$ & \\
\hline \multirow[t]{2}{*}{ Gender (\% male) } & Median $38.9 \%$ (range $25 \%$ - 98\%) & Median $49.8 \%$ (range $25 \%$ - 97\%): & Median $48 \%$ (25\% - 98\%) \\
\hline & Number reporting $\mathrm{n}=12$ & Number reporting $n=35$ & Number reporting $n=47$ \\
\hline \multirow[t]{4}{*}{ Type of screening } & Retinal exam & Retinal exam & Retinal exam \\
\hline & $n=12(75 \%)$ & $n=49(98 \%)$ & $\mathrm{n}=61(92.4 \%)$ \\
\hline & $\begin{array}{l}\text { Grading of digital retinal images: } n= \\
4(25 \%)\end{array}$ & Grading of retinal images & Grading of retinal images \\
\hline & & $\mathrm{n}=1(2 \%)$ & $\mathrm{n}=5(7.6 \%)$ \\
\hline \multirow{2}{*}{$\begin{array}{l}\text { Baseline screening } \\
\text { attendance (in pre- } \\
\text { vious } 12 \text { or } 24 \mathrm{~m} \text { ) }\end{array}$} & Median $0 \%$ (range $0 \%-48.4 \%$ ) & Median $37.1 \%$ (range $0 \%$ - 88\%) & Median $35.4 \%$ (range $0 \%-87.8 \%$ ) \\
\hline & Reported in 7 studies & Reported in 36 studies & Reported in 43 studies \\
\hline \multirow{3}{*}{$\begin{array}{l}\text { Longest duration } \\
\text { of follow-up (medi- } \\
\text { an)* }\end{array}$} & Median 6 months & Median 12 months & Median 12 months \\
\hline & (range $3-48$ ) & (range 1 - 30): & (range $1-48$ ) \\
\hline & Number reporting $n=14$ & Number reporting $n=49$ & Number reporting $n=63$ \\
\hline \multirow{5}{*}{$\begin{array}{l}\text { Intervention tar- } \\
\text { get (modified } \\
\text { EPOC classifica- } \\
\text { tion) }\end{array}$} & $\begin{array}{l}\text { Median number of targets in inter- } \\
\text { vention } \mathrm{arm}=2\end{array}$ & $\begin{array}{l}\text { Median number of targets in in- } \\
\text { tervention } \text { arm }=3\end{array}$ & $\begin{array}{l}\text { Median number of targets in in- } \\
\text { tervention } \operatorname{arm}=3\end{array}$ \\
\hline & Participant n = $14(87.5 \%)$ & Participant n = 31 (62\%) & Participant n = 45 (68.2\%) \\
\hline & Healthcare professional $n=4(25 \%)$ & Healthcare professional $n=31$ & Healthcare professional $n=35$ \\
\hline & Healthcare system n = 4 (25\%) & & \\
\hline & & Healthcare system $n=37(74 \%)$ & Healthcare system $n=41(62.1 \%)$ \\
\hline
\end{tabular}

Mansberger 2015 reported follow-up data to 48 months but intervention offered to intervention and control group after 18 months and data reported at 12 and 24 months. 


\begin{tabular}{|c|c|c|c|c|c|c|c|c|c|c|c|c|c|c|}
\hline $\begin{array}{l}\text { CHEC criteria check- } \\
\text { lists }\end{array}$ & $\begin{array}{l}\text { Adair } \\
2013\end{array}$ & $\begin{array}{l}\text { Clancy } \\
2007\end{array}$ & $\begin{array}{l}\text { Davis } \\
2011\end{array}$ & $\begin{array}{l}\text { Eccles } \\
2007\end{array}$ & $\begin{array}{l}\text { Frei } \\
2014\end{array}$ & $\begin{array}{l}\text { Fri- } \\
\text { jling } \\
2002\end{array}$ & $\begin{array}{l}\text { Krein } \\
2004\end{array}$ & $\begin{array}{l}\text { Litak- } \\
\text { er } \\
2003\end{array}$ & $\begin{array}{l}\text { McCall } \\
2011\end{array}$ & $\begin{array}{l}\text { Piette } \\
2001\end{array}$ & $\begin{array}{l}\text { Pizzi } \\
2015\end{array}$ & $\begin{array}{l}\text { Prezio } \\
2014\end{array}$ & $\begin{array}{l}\text { Schec } \\
2008\end{array}$ & $\begin{array}{l}\text { rWag } \\
\text { ner } \\
2001\end{array}$ \\
\hline $\begin{array}{l}\text { Is the study population } \\
\text { clearly described? }\end{array}$ & $Y$ & $Y$ & $Y$ & $Y$ & $Y$ & $Y$ & $Y$ & $Y$ & $Y$ & $Y$ & $Y$ & $Y$ & $Y$ & $Y$ \\
\hline $\begin{array}{l}\text { Are competing alterna- } \\
\text { tives clearly described? }\end{array}$ & $Y$ & $Y$ & $Y$ & $\mathrm{~N}$ & $\mathrm{~N}$ & $\mathrm{Y}$ & $Y$ & $Y$ & $\mathrm{~N}$ & $\mathrm{~N}$ & $Y$ & $Y$ & $Y$ & $\mathrm{~N}$ \\
\hline $\begin{array}{l}\text { Is a well-defined re- } \\
\text { search question posed } \\
\text { in answerable form? }\end{array}$ & $Y$ & $Y$ & $Y$ & $Y$ & $Y$ & $Y$ & $Y$ & $Y$ & $Y$ & $\mathrm{~N}$ & $Y$ & $Y$ & $Y$ & $Y$ \\
\hline $\begin{array}{l}\text { Is the economic study } \\
\text { design appropriate to } \\
\text { the stated objective? }\end{array}$ & $\mathrm{N}$ & $\mathrm{N}$ & $Y$ & $\mathrm{~N}$ & $\mathrm{~N}$ & $\mathrm{~N}$ & $\mathrm{~N}$ & $Y$ & $\mathrm{~N}$ & $\mathrm{~N}$ & $Y$ & $Y$ & $Y$ & $\mathrm{~N}$ \\
\hline $\begin{array}{l}\text { Is the chosen time hori- } \\
\text { zon appropriate to in- } \\
\text { clude relevant costs } \\
\text { and consequences? }\end{array}$ & $Y$ & $\mathrm{~N}$ & $U$ & $\mathrm{~N}$ & $\mathrm{~N}$ & $\mathrm{~N}$ & $\mathrm{~N}$ & $Y$ & $\mathrm{~N}$ & $\mathrm{~N}$ & $Y$ & $Y$ & $Y$ & $\mathrm{~N}$ \\
\hline $\begin{array}{l}\text { Is the actual perspec- } \\
\text { tive chosen appropri- } \\
\text { ate? }\end{array}$ & $Y$ & $\mathrm{~N}$ & $Y$ & $Y$ & $Y$ & Y & $Y$ & $Y$ & $Y$ & $Y$ & $Y$ & $Y$ & $Y$ & $Y$ \\
\hline $\begin{array}{l}\text { Are all important and } \\
\text { relevant costs for each } \\
\text { alternative identified? }\end{array}$ & $Y$ & $\mathrm{~N}$ & $Y$ & $Y$ & $\mathrm{~N}$ & $\mathrm{~N}$ & $\mathrm{~N}$ & $\mathrm{~N}$ & $\mathrm{~N}$ & $\mathrm{~N}$ & $Y$ & $Y$ & $Y$ & $\mathrm{~N}$ \\
\hline $\begin{array}{l}\text { Are all costs measured } \\
\text { appropriately in physi- } \\
\text { cal units? }\end{array}$ & $Y$ & $\mathrm{~N}$ & $U$ & $Y$ & $\mathrm{~N}$ & $Y$ & $\mathrm{~N}$ & $Y$ & $Y$ & $\mathrm{~N}$ & $Y$ & $Y$ & $Y$ & $\mathrm{~N}$ \\
\hline $\begin{array}{l}\text { Are costs valued appro- } \\
\text { priately? }\end{array}$ & $Y$ & $\mathrm{~N}$ & $\mathrm{~N}$ & $Y$ & $\mathrm{~N}$ & $Y$ & $\mathrm{~N}$ & $Y$ & $\mathrm{~N}$ & $\mathrm{~N}$ & $Y$ & $Y$ & $Y$ & $\mathrm{~N}$ \\
\hline $\begin{array}{l}\text { Are all important and } \\
\text { relevant outcomes for } \\
\text { each alternative identi- } \\
\text { fied? }\end{array}$ & $Y$ & $\mathrm{~N}$ & $Y$ & $Y$ & $Y$ & $Y$ & $\mathrm{~N}$ & $Y$ & $\mathrm{~N}$ & $Y$ & $Y$ & $Y$ & $Y$ & $\mathrm{~N}$ \\
\hline
\end{tabular}




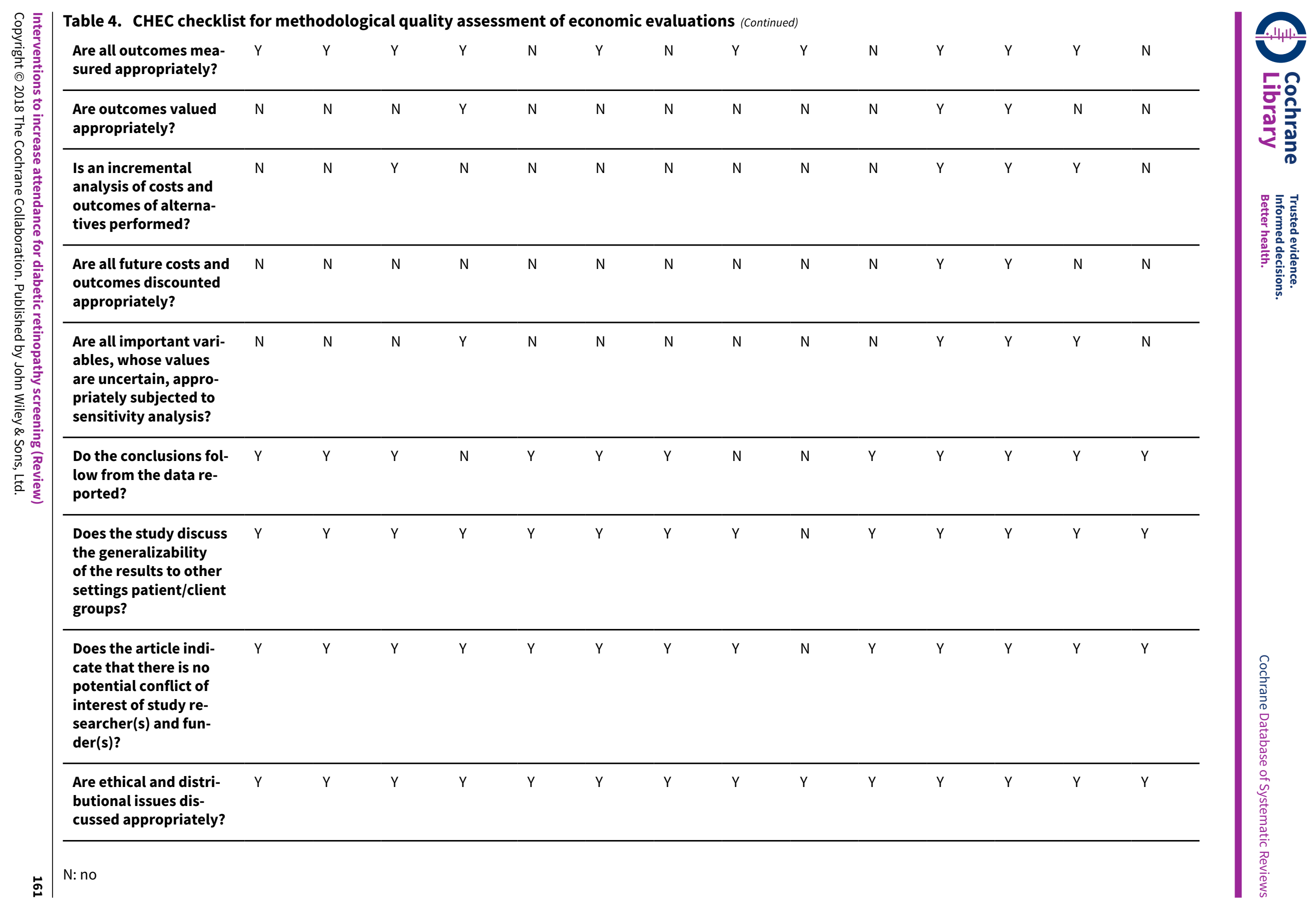



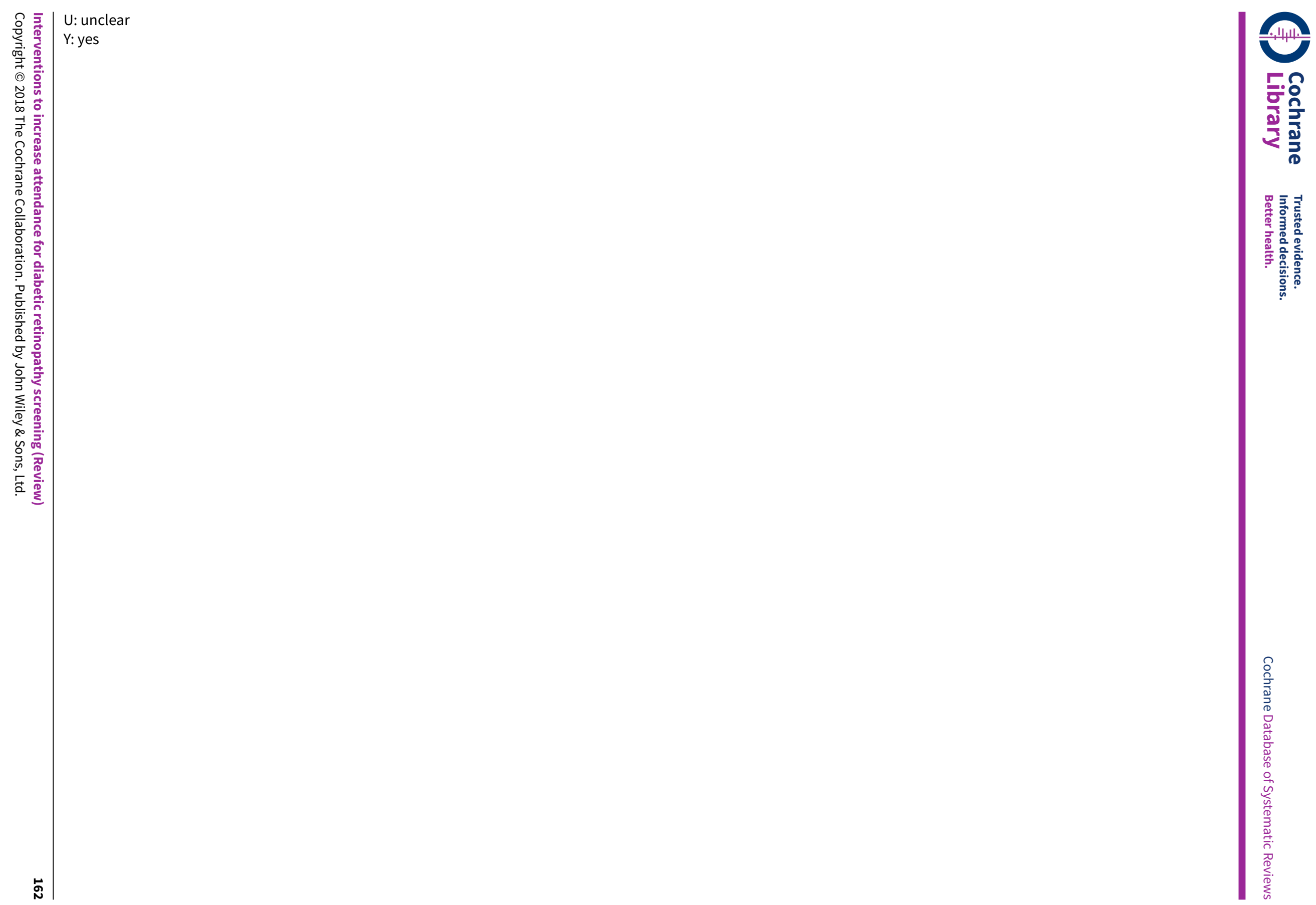
Table 5. CHEERS checklist for methodological quality assessment of economic evaluations

\begin{tabular}{lll}
\hline Section of paper Component & $\begin{array}{l}\text { Reported on page } \\
\text { number }\end{array}$ \\
\hline
\end{tabular}

\section{Adair 2013}

\begin{tabular}{lll}
\hline Abstract & $\begin{array}{l}\text { Provide a structured summary of objectives, perspective, setting, methods (in- } \\
\text { cluding study design and inputs), results (including base case and uncertainty } \\
\text { analyses), and conclusions }\end{array}$ & Not reported \\
\hline Introduction & & 176 \\
\hline $\begin{array}{l}\text { Background and ob- } \\
\text { jectives }\end{array}$ & $\begin{array}{l}\text { Provide an explicit statement of the broader context for the study } \\
\text { sions }\end{array}$ & 176 \\
\cline { 2 - 4 }
\end{tabular}

\section{Methods}

\begin{tabular}{lll}
\hline $\begin{array}{l}\text { Target population and } \\
\text { subgroups }\end{array}$ & $\begin{array}{l}\text { Describe characteristics of the base case population and subgroups analysed, } \\
\text { including why they were chosen }\end{array}$ & 177 \\
\hline
\end{tabular}

\begin{tabular}{lll}
\hline Setting and location & $\begin{array}{l}\text { State relevant aspects of the system(s) in which the decision(s) need(s) to be } \\
\text { made }\end{array}$ & 177
\end{tabular}

\begin{tabular}{ll}
\hline Study perspective & $\begin{array}{l}\text { Describe the perspective of the study and relate this to the costs being evaluat- } \\
\text { ed }\end{array}$
\end{tabular}

\begin{tabular}{|c|c|c|}
\hline Comparators & $\begin{array}{l}\text { Describe the interventions or strategies being compared and state why they } \\
\text { were chosen }\end{array}$ & Not reported \\
\hline Time horizon & $\begin{array}{l}\text { State the time horizon(s) over which costs and consequences are being evalu- } \\
\text { ated and say why appropriate }\end{array}$ & Not reported \\
\hline Discount rate & $\begin{array}{l}\text { Report the choice of discount rate(s) used for costs and outcomes and say why } \\
\text { appropriate }\end{array}$ & Not reported \\
\hline $\begin{array}{l}\text { Choice of health out- } \\
\text { comes }\end{array}$ & $\begin{array}{l}\text { Describe what outcomes were used as the measure(s) of benefit in the evalua- } \\
\text { tion and their relevance for the type of analysis performed }\end{array}$ & Not reported \\
\hline \multirow[t]{2}{*}{$\begin{array}{l}\text { Measurement of effec- } \\
\text { tiveness }\end{array}$} & $\begin{array}{l}\text { Single study-based estimates: Describe fully the design features of the single } \\
\text { effectiveness study and why the single study was a sufficient source of clinical } \\
\text { effectiveness data }\end{array}$ & Not reported \\
\hline & $\begin{array}{l}\text { Synthesis-based estimates: Describe fully the methods used for identification } \\
\text { of included studies and synthesis of clinical effectiveness data }\end{array}$ & Not reported \\
\hline $\begin{array}{l}\text { Measurement and val- } \\
\text { uation of preference } \\
\text { based outcomes }\end{array}$ & $\begin{array}{l}\text { If applicable, describe the population and methods used to elicit preferences } \\
\text { for outcomes }\end{array}$ & Not reported \\
\hline $\begin{array}{l}\text { Estimating resources } \\
\text { and costs }\end{array}$ & $\begin{array}{l}\text { Single study-based economic evaluation: Describe approaches used to esti- } \\
\text { mate resource use associated with the alternative interventions. Describe pri- } \\
\text { mary or secondary research methods for valuing each resource item in terms } \\
\text { of its unit cost. Describe any adjustments made to approximate to opportunity } \\
\text { costs }\end{array}$ & 179 \\
\hline
\end{tabular}


Table 5. CHEERS checklist for methodological quality assessment of economic evaluations (Continued)

Currency, price date, Report the dates of the estimated resource quantities and unit costs. Describe and conversion methods for adjusting estimated unit costs to the year of reported costs if necessary. Describe methods for converting costs into a common currency base and the exchange rate

\begin{tabular}{llll}
\hline Choice of model & $\begin{array}{l}\text { Describe and give reasons for the specific type of decision-analytical model } \\
\text { used. Providing a figure to show model structure is strongly recommended }\end{array}$ & Not reported \\
\hline Assumptions & $\begin{array}{l}\text { Describe all structural or other assumptions underpinning the decision-analyt- } \\
\text { ical model }\end{array}$ & Not reported
\end{tabular}

\begin{tabular}{ll}
\hline Analytical methods & $\begin{array}{l}\text { Describe all analytical methods supporting the evaluation. This could include } \\
\text { methods for dealing with skewed, missing, or censored data; extrapolation } \\
\text { methods; methods for pooling data; approaches to validate or make adjust- } \\
\text { ments (such as half cycle corrections) to a model; and methods for handling } \\
\text { population heterogeneity and uncertainty }\end{array}$
\end{tabular}

\section{Results}

\begin{tabular}{lll}
\hline Study parameters & $\begin{array}{l}\text { Report the values, ranges, references, and, if used, probability distributions for } \\
\text { all parameters. Report reasons or sources for distributions used to represent } \\
\text { uncertainty where appropriate. Providing a table to show the input values is } \\
\text { strongly recommended }\end{array}$ & w65
\end{tabular}

$\begin{array}{ll}\begin{array}{l}\text { Incremental costs and } \\ \text { outcomes }\end{array} & \begin{array}{l}\text { For each intervention, report mean values for the main categories of estimated } \\ \text { costs and outcomes of interest, as well as mean differences between the com- } \\ \text { parator groups. If applicable, report incremental cost-effectiveness ratios }\end{array}\end{array}$

Characterising uncer-
tainty

Single study-based economic evaluation: Describe the effects of sampling uncertainty for the estimated incremental cost and incremental effectiveness pa-

Appendices w65 rameters, together with the impact of methodological assumptions (such as discount rate, study perspective)

Model-based economic evaluation: Describe the effects on the results of uncertainty for all input parameters, and uncertainty related to the structure of the model and assumptions

Characterising hetero-
geneity

If applicable, report differences in costs, outcomes, or cost-effectiveness that can be explained by variations between subgroups of patients with different baseline characteristics or other observed variability in effects that are not reducible by more information

\section{Discussion}

Not reported

Not reported

Not reported

\footnotetext{
Study findings, limitations, generalisabili-

Summarise key study findings and describe how they support the conclusions

ty, and current knowlreached. Discuss limitations and the generalisability of the findings and how the findings fit with current knowledge edge
}

183

\section{Other}

Source of funding Describe how the study was funded and the role of the funder in the identification, design, conduct, and reporting of the analysis. Describe other non-monetary sources of support

\section{Conflicts of interest}


Table 5. CHEERS checklist for methodological quality assessment of economic evaluations (Continued)

authors comply with International Committee of Medical Journal Editors rec-

ommendations

\section{Clancy 2007}

\begin{tabular}{ll}
\hline Title & $\begin{array}{l}\text { Identify the study as an economic evaluation or use more specific terms such } \\
\text { as "cost-effectiveness analysis", and describe the interventions compared. }\end{array}$
\end{tabular}

\begin{tabular}{ll}
\hline Abstract & $\begin{array}{l}\text { Provide a structured summary of objectives, perspective, setting, methods (in- Not reported } \\
\text { cluding study design and inputs), results (including base case and uncertainty } \\
\text { analyses), and conclusions. }\end{array}$
\end{tabular}

\section{Introduction}

\begin{tabular}{lll}
\hline $\begin{array}{l}\text { Background and ob- } \\
\text { jectives }\end{array}$ & $\begin{array}{l}\text { Provide an explicit statement of the broader context for the study. } \\
\text { Not reported }\end{array}$ \\
\cline { 2 - 3 } & $\begin{array}{l}\text { Present the study question and its relevance for health policy or practice deci- } \\
\text { sions. }\end{array}$ & 620
\end{tabular}

\begin{tabular}{lll}
\hline Methods & & Not reported \\
\hline $\begin{array}{l}\text { Target population and } \\
\text { subgroups }\end{array}$ & $\begin{array}{l}\text { Describe characteristics of the base case population and subgroups analysed, } \\
\text { including why they were chosen. }\end{array}$ & 621 \\
\hline Setting and location & $\begin{array}{l}\text { State relevant aspects of the system(s) in which the decision(s) need(s) to be } \\
\text { made. }\end{array}$ & Not reported
\end{tabular}

\begin{tabular}{ll}
\hline Study perspective & $\begin{array}{l}\text { Describe the perspective of the study and relate this to the costs being evaluat- Not reported } \\
\text { ed. }\end{array}$
\end{tabular}

\begin{tabular}{ll}
\hline Comparators & $\begin{array}{l}\text { Describe the interventions or strategies being compared and state why they } \\
\text { were chosen. }\end{array}$
\end{tabular}

\begin{tabular}{ll}
\hline Time horizon & $\begin{array}{l}\text { State the time horizon(s) over which costs and consequences are being evalu- } \\
\text { ated and say why appropriate. }\end{array}$
\end{tabular}

\begin{tabular}{ll}
\hline Discount rate & $\begin{array}{l}\text { Report the choice of discount rate(s) used for costs and outcomes and say why Not reported } \\
\text { appropriate. }\end{array}$
\end{tabular}

\begin{tabular}{lll}
\hline $\begin{array}{l}\text { Choice of health out- } \\
\text { comes }\end{array}$ & $\begin{array}{l}\text { Describe what outcomes were used as the measure(s) of benefit in the evalua- } \\
\text { tion and their relevance for the type of analysis performed. }\end{array}$ & Not reported
\end{tabular}

\begin{tabular}{ll}
\hline $\begin{array}{l}\text { Measurement of effec- } \\
\text { tiveness }\end{array}$ & $\begin{array}{l}\text { Single study-based estimates: Describe fully the design features of the single } \\
\text { effectiveness study and why the single study was a sufficient source of clinical } \\
\text { effectiveness data. }\end{array}$
\end{tabular}

Synthesis-based estimates: Describe fully the methods used for identification Not reported of included studies and synthesis of clinical effectiveness data.

\begin{tabular}{ll}
\hline $\begin{array}{l}\text { Measurement and val- } \\
\text { uation of preference } \\
\text { based outcomes }\end{array}$ & $\begin{array}{l}\text { If applicable, describe the population and methods used to elicit preferences } \\
\text { for outcomes. }\end{array}$ \\
\hline $\begin{array}{l}\text { Estimating resources } \\
\text { and costs }\end{array}$ & $\begin{array}{l}\text { Single study-based economic evaluation: Describe approaches used to esti- } \\
\text { mate resource use associated with the alternative interventions. Describe pri- } \\
\text { mary or secondary research methods for valuing each resource item in terms }\end{array}$
\end{tabular}


Table 5. CHEERS checklist for methodological quality assessment of economic evaluations (Continued)

of its unit cost. Describe any adjustments made to approximate to opportunity costs.

\section{Currency, price date, and conversion}

Report the dates of the estimated resource quantities and unit costs. Describe methods for adjusting estimated unit costs to the year of reported costs if necessary. Describe methods for converting costs into a common currency base and the exchange rate.
Not reported

Not reported

\begin{tabular}{lll}
\hline Choice of model & $\begin{array}{l}\text { Describe and give reasons for the specific type of decision-analytical model } \\
\text { used. Providing a figure to show model structure is strongly recommended. }\end{array} \quad$ Not reported
\end{tabular}

\begin{tabular}{lll}
\hline Assumptions & $\begin{array}{l}\text { Describe all structural or other assumptions underpinning the decision-analyt- } \\
\text { ical model. }\end{array}$ & Not reported \\
\hline Analytical methods & $\begin{array}{l}\text { Describe all analytical methods supporting the evaluation. This could include } \\
\text { methods for dealing with skewed, missing, or censored data; extrapolation } \\
\text { methods; methods for pooling data; approaches to validate or make adjust- } \\
\text { ments (such as half cycle corrections) to a model; and methods for handling } \\
\text { population heterogeneity and uncertainty. }\end{array}$ & 622 \\
&
\end{tabular}

Results

$\begin{array}{ll}\text { Study parameters } & \text { Report the values, ranges, references, and, if used, probability distributions for } \\ \text { all parameters. Report reasons or sources for distributions used to represent } \\ \text { uncertainty where appropriate. Providing a table to show the input values is } \\ \text { strongly recommended. }\end{array}$

\section{Incremental costs and outcomes}

For each intervention, report mean values for the main categories of estimated costs and outcomes of interest, as well as mean differences between the comparator groups. If applicable, report incremental cost-effectiveness ratios.
Not reported

Single study-based economic evaluation: Describe the effects of sampling uncertainty for the estimated incremental cost and incremental effectiveness parameters, together with the impact of methodological assumptions (such as discount rate, study perspective).
Not reported
Characterising uncertainty
Model-based economic evaluation: Describe the effects on the results of uncertainty for all input parameters, and uncertainty related to the structure of the model and assumptions

\section{Characterising hetero- geneity
If applicable, report differences in costs, outcomes, or cost-effectiveness that can be explained by variations between subgroups of patients with different baseline characteristics or other observed variability in effects that are not re- ducible by more information.

Not reported

Not reported

\section{Discussion}

Not reported reached. Discuss limitations and the generalisability of the findings and how the findings fit with current knowledge. tions, generalisabili-

ty, and current knowledge

\section{Other}

Source of funding Describe how the study was funded and the role of the funder in the identification, design, conduct, and reporting of the analysis. Describe other non-monetary sources of support. 
Table 5. CHEERS checklist for methodological quality assessment of economic evaluations (Continued)

Conflicts of interest Describe any potential for conflict of interest of study contributors in accor-

624 dance with journal policy. In the absence of a journal policy, we recommend authors comply with International Committee of Medical Journal Editors recommendations.

\section{Davis 2010}

Title Identify the study as an economic evaluation or use more specific terms such Abstract
as "cost-effectiveness analysis", and describe the interventions compared.

Abstract

Provide a structured summary of objectives, perspective, setting, methods (including study design and inputs), results (including base case and uncertainty analyses), and conclusions.

\section{Abstract}

A325

\section{Introduction}

\begin{tabular}{lll}
\hline $\begin{array}{l}\text { Background and ob- } \\
\text { jectives }\end{array}$ & Provide an explicit statement of the broader context for the study. & Abstract \\
\cline { 2 - 3 } & $\begin{array}{l}\text { Present the study question and its relevance for health policy or practice deci- } \\
\text { sions. }\end{array}$ & $\begin{array}{l}1712 \text { of effectiveness re- } \\
\text { port }\end{array}$ \\
\hline
\end{tabular}

\section{Methods}

\section{Target population and subgroups}

\section{Setting and location} Describe characteristics of the bas
including why they were chosen.

Describe characteristics of the base case population and subgroups analysed, State relevant aspects of the system(s) in which the decision(s) need(s) to be made.

1714 of effectiveness report

Abstract

\section{Study perspective}

Describe the perspective of the study and relate this to the costs being evaluated.

\begin{tabular}{ll}
\hline Comparators & $\begin{array}{l}\text { Describe the interventions or strategies being compared and state why they } \\
\text { were chosen. }\end{array}$ \\
\hline Time horizon & $\begin{array}{l}\text { State the time horizon(s) over which costs and consequences are being evalu- } \\
\text { ated and say why appropriate. }\end{array}$
\end{tabular}

\begin{tabular}{llll}
\hline Discount rate & $\begin{array}{l}\text { Report the choice of discount rate(s) used for costs and outcomes and say why } \\
\text { appropriate. }\end{array}$ & Not reported \\
\hline $\begin{array}{l}\text { Choice of health out- } \\
\text { comes }\end{array}$ & $\begin{array}{l}\text { Describe what outcomes were used as the measure(s) of benefit in the evalua- } \\
\text { tion and their relevance for the type of analysis performed. }\end{array}$ & 1713 \\
\hline $\begin{array}{l}\text { Measurement of effec- } \\
\text { tiveness }\end{array}$ & $\begin{array}{l}\text { Single study-based estimates: Describe fully the design features of the single } \\
\text { effectiveness study and why the single study was a sufficient source of clinical } \\
\text { effectiveness data. }\end{array}$ & Abstract & A325 \\
\cline { 2 - 3 } & $\begin{array}{l}\text { Synthesis-based estimates: Describe fully the methods used for identification } \\
\text { of included studies and synthesis of clinical effectiveness data. }\end{array}$ & Not applicable \\
\hline
\end{tabular}

\section{A325}

Abstract

A325 
Table 5. CHEERS checklist for methodological quality assessment of economic evaluations (Continued)

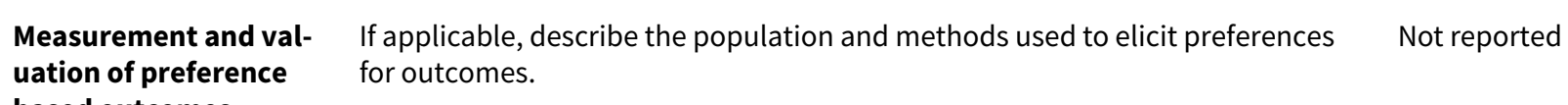
uation of preference for outcomes.

based outcomes

$\begin{array}{ll}\text { Estimating resources } & \text { Single study-based economic evaluation: Describe approaches used to esti- } \\ \text { and costs } & \text { mate resource use associated with the alternative interventions. Describe pri- } \\ & \text { mary or secondary research methods for valuing each resource item in terms } \\ & \text { of its unit cost. Describe any adjustments made to approximate to opportunity } \\ & \text { costs. }\end{array}$

Not reported

of its unit cost. Describe any adjustments made to approximate to opportunity costs.

Currency, price date,
and conversion

Report the dates of the estimated resource quantities and unit costs. Describe methods for adjusting estimated unit costs to the year of reported costs if necessary. Describe methods for converting costs into a common currency base and the exchange rate.
Not reported

Not applicable

Choice of model Describe and give reasons for the specific type of decision-analytical model
used. Providing a figure to show model structure is strongly recommended.

Assumptions Describe all structural or other assumptions underpinning the decision-analyt- Not applicable
ical model.

\begin{tabular}{ll}
\hline Analytical methods & Describe all analytical methods supporting the evaluation. This could include \\
methods for dealing with skewed, missing, or censored data; extrapolation \\
methods; methods for pooling data; approaches to validate or make adjust- \\
ments (such as half cycle corrections) to a model; and methods for handling \\
population heterogeneity and uncertainty.
\end{tabular}

Not applicable

Results

Study parameters
Report the values, ranges, references, and, if used, probability distributions for all parameters. Report reasons or sources for distributions used to represent uncertainty where appropriate. Providing a table to show the input values is strongly recommended.
Not reported

For each intervention, report mean values for the main categories of estimated costs and outcomes of interest, as well as mean differences between the comparator groups. If applicable, report incremental cost-effectiveness ratios.

Abstract

A325

Not reported

Single study-based economic evaluation: Describe the effects of sampling uncertainty for the estimated incremental cost and incremental effectiveness parameters, together with the impact of methodological assumptions (such as discount rate, study perspective).

\section{Characterising uncer- tainty}

Model-based economic evaluation: Describe the effects on the results of uncertainty for all input parameters, and uncertainty related to the structure of the model and assumptions.
Not applicable

Not reported
Characterising hetero- geneity
If applicable, report differences in costs, outcomes, or cost-effectiveness that can be explained by variations between subgroups of patients with different baseline characteristics or other observed variability in effects that are not re- ducible by more information.

\section{Discussion}

\section{Study findings, limita-} tions, generalisability, and current knowledge

Summarise key study findings and describe how they support the conclusions reached. Discuss limitations and the generalisability of the findings and how the findings fit with current knowledge.
}

Not reported 
Table 5. CHEERS checklist for methodological quality assessment of economic evaluations (Continued)

Other

Source of funding Describe how the study was funded and the role of the funder in the identifica-

tion, design, conduct, and reporting of the analysis. Describe other non-monetary sources of support.

Conflicts of interest

Describe any potential for conflict of interest of study contributors in accordance with journal policy. In the absence of a journal policy, we recommend authors comply with International Committee of Medical Journal Editors recommendations.

\section{Eccles 2007}

Title
as "contify the study as an economic evaluation or use more specific terms such Noness analysis", and describe the interventions compared.

$\begin{array}{ll}\text { Abstract } & \begin{array}{l}\text { Provide a structured summary of objectives, perspective, setting, methods (in- } \\ \text { cluding study design and inputs), results (including base case and uncertainty } \\ \text { analyses), and conclusions. }\end{array}\end{array}$

\section{Introduction}

Background and objectives
Provide an explicit statement of the broader context for the study.

Present the study question and its relevance for health policy or practice decisions.

\section{Methods} $\begin{array}{lll}\begin{array}{l}\text { Target population and } \\ \text { subgroups }\end{array} & \begin{array}{l}\text { Describe characteristics of the base case population and subgroups analysed, } \\ \text { including why they were chosen. }\end{array} & 2\end{array}$

Setting and location State relevant aspects of the system(s) in which the decision(s) need(s) to be 2
made.

Study perspective $\quad$ Describe the perspective of the study and relate this to the costs being evaluat- 4
ed.

\begin{tabular}{|c|c|c|}
\hline Comparators & $\begin{array}{l}\text { Describe the interventions or strategies being compared and state why they } \\
\text { were chosen. }\end{array}$ & 4 \\
\hline Time horizon & $\begin{array}{l}\text { State the time horizon(s) over which costs and consequences are being evalu- } \\
\text { ated and say why appropriate. }\end{array}$ & 4 \\
\hline Discount rate & $\begin{array}{l}\text { Report the choice of discount rate(s) used for costs and outcomes and say why } \\
\text { appropriate. }\end{array}$ & \\
\hline $\begin{array}{l}\text { Choice of health out- } \\
\text { comes }\end{array}$ & $\begin{array}{l}\text { Describe what outcomes were used as the measure(s) of benefit in the evalua- } \\
\text { tion and their relevance for the type of analysis performed. }\end{array}$ & 3 \\
\hline $\begin{array}{l}\text { Measurement of effec- } \\
\text { tiveness }\end{array}$ & $\begin{array}{l}\text { Single study-based estimates: Describe fully the design features of the single } \\
\text { effectiveness study and why the single study was a sufficient source of clinical } \\
\text { effectiveness data. }\end{array}$ & Not reported \\
\hline
\end{tabular}


Table 5. CHEERS checklist for methodological quality assessment of economic evaluations (Continued)

Synthesis-based estimates: Describe fully the methods used for identification Not reported of included studies and synthesis of clinical effectiveness data.

\begin{tabular}{ll}
\hline $\begin{array}{l}\text { Measurement and val- } \\
\text { uation of preference } \\
\text { based outcomes }\end{array}$ & $\begin{array}{l}\text { If applicable, describe the population and methods used to elicit preferences } \\
\text { for outcomes. }\end{array}$ \\
\hline $\begin{array}{l}\text { Estimating resources } \\
\text { and costs }\end{array}$ & $\begin{array}{l}\text { Single study-based economic evaluation: Describe approaches used to esti- } \\
\text { mate resource use associated with the alternative interventions. Describe pri- } \\
\text { mary or secondary research methods for valuing each resource item in terms } \\
\text { of its unit cost. Describe any adjustments made to approximate to opportunity } \\
\text { costs. }\end{array}$
\end{tabular}

\section{Currency, price date, and conversion}

Report the dates of the estimated resource quantities and unit costs. Describe methods for adjusting estimated unit costs to the year of reported costs if necessary. Describe methods for converting costs into a common currency base and the exchange rate.
3

3

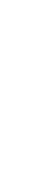

\section{Describe and give reasons for the specific type of decision-analytical model} used. Providing a figure to show model structure is strongly recommended.

\begin{tabular}{lll}
\hline Choice of model & $\begin{array}{l}\text { Describe and give reasons for the specific type of decision-analytical model } \\
\text { used. Providing a figure to show model structure is strongly recommended. }\end{array}$ & Not reported \\
\hline Assumptions & $\begin{array}{l}\text { Describe all structural or other assumptions underpinning the decision-analyt- } \\
\text { ical model. }\end{array}$ & Not reported \\
\hline Analytical methods & $\begin{array}{l}\text { Describe all analytical methods supporting the evaluation. This could include } \\
\text { methods for dealing with skewed, missing, or censored data; extrapolation } \\
\text { methods; methods for pooling data; approaches to validate or make adjust- } \\
\text { ments (such as half cycle corrections) to a model; and methods for handling } \\
\text { population heterogeneity and uncertainty. }\end{array}$ \\
\hline
\end{tabular}

\section{Results}

\begin{tabular}{ll}
\hline Study parameters & Report the values, ranges, references, and, if used, probability distributions for \\
& all parameters. Report reasons or sources for distributions used to represent \\
& uncertainty where appropriate. Providing a table to show the input values is \\
& strongly recommended.
\end{tabular}

Not reportted uncertainty where appropriate. Providing a table to show the input values is strongly recommended.

Incremental costs and
outcomes

For each intervention, report mean values for the main categories of estimated costs and outcomes of interest, as well as mean differences between the comparator groups. If applicable, report incremental cost-effectiveness ratios.
Characterising uncer- tainty
Single study-based economic evaluation: Describe the effects of sampling un- certainty for the estimated incremental cost and incremental effectiveness pa- rameters, together with the impact of methodological assumptions (such as discount rate, study perspective). Not reported

Model-based economic evaluation: Describe the effects on the results of uncerNot reported tainty for all input parameters, and uncertainty related to the structure of the model and assumptions.

\begin{tabular}{ll}
\hline Characterising hetero- & $\begin{array}{l}\text { If applicable, report differences in costs, outcomes, or cost-effectiveness that } \\
\text { geneity }\end{array}$ \\
& $\begin{array}{l}\text { can be explained by variations between subgroups of patients with different } \\
\text { ducible by moracteristics or other observed variability in effects that are not re- }\end{array}$ \\
&
\end{tabular}

Not reported baseline characteristics or other observed variability in effects that are not reducible by more information.

\section{Discussion}


Table 5. CHEERS checklist for methodological quality assessment of economic evaluations

(Continued)

$\begin{array}{ll}\text { Study findings, limita- } & \text { Summarise key study findings and describe how they support the conclusions } \\ \text { tions, generalisabili- } & \text { reached. Discuss limitations and the generalisability of the findings and how } \\ \text { ty, and current knowl- } & \text { the findings fit with current knowledge. }\end{array}$

6, 10

edge

\section{Other}

Source of funding

Describe how the study was funded and the role of the funder in the identification, design, conduct, and reporting of the analysis. Describe other non-monetary sources of support.

Conflicts of interest Describe any potential for conflict of interest of study contributors in accordance with journal policy. In the absence of a journal policy, we recommend authors comply with International Committee of Medical Journal Editors recommendations.

\section{Frei 2014}

\begin{tabular}{lll}
\hline Title & $\begin{array}{l}\text { Identify the study as an economic evaluation or use more specific terms such } \\
\text { as "cost-effectiveness analysis", and describe the interventions compared. }\end{array}$ & Not reported \\
\hline Abstract & $\begin{array}{l}\text { Provide a structured summary of objectives, perspective, setting, methods (in- } \\
\text { cluding study design and inputs), results (including base case and uncertainty } \\
\text { analyses), and conclusions. }\end{array}$ & Not reported \\
\hline Introduction & Provide an explicit statement of the broader context for the study. & 1040 \\
\hline $\begin{array}{l}\text { Background and ob- } \\
\text { jectives }\end{array}$ & $\begin{array}{l}\text { Present the study question and its relevance for health policy or practice deci- } \\
\text { sions. }\end{array}$ & 1040 \\
\hline
\end{tabular}

\section{Methods}
Target population and Describe characteristics of the base case population and subgroups analysed, 1043 subgroups including why they were chosen.

\begin{tabular}{lll}
\hline Setting and location & $\begin{array}{l}\text { State relevant aspects of the system(s) in which the decision(s) need(s) to be } \\
\text { made. }\end{array}$ & 1040
\end{tabular}

\begin{tabular}{ll}
\hline Study perspective & Describe the perspective of the study and relate this to the costs being evaluat- Not reported \\
ed.
\end{tabular}

\begin{tabular}{lll}
\hline Comparators & $\begin{array}{l}\text { Describe the interventions or strategies being compared and state why they } \\
\text { were chosen. }\end{array}$ & 1040 \\
\hline Time horizon & $\begin{array}{l}\text { State the time horizon(s) over which costs and consequences are being evalu- } \\
\text { ated and say why appropriate. }\end{array}$ & Not reported \\
\hline Discount rate & $\begin{array}{l}\text { Report the choice of discount rate(s) used for costs and outcomes and say why } \\
\text { appropriate. }\end{array}$ & Not reported \\
\hline $\begin{array}{l}\text { Choice of health out- } \\
\text { comes }\end{array}$ & $\begin{array}{l}\text { Describe what outcomes were used as the measure(s) of benefit in the evalua- } \\
\text { tion and their relevance for the type of analysis performed. }\end{array}$ & Not reported \\
\hline
\end{tabular}


Table 5. CHEERS checklist for methodological quality assessment of economic evaluations (Continued) $\begin{array}{ll}\begin{array}{l}\text { Measurement of effec- } \\ \text { tiveness }\end{array} & \begin{array}{l}\text { Single study-based estimates: Describe fully the design features of the single } \\ \text { effectiveness study and why the single study was a sufficient source of clinical } \\ \text { effectiveness data. }\end{array}\end{array}$

Synthesis-based estimates: Describe fully the methods used for identification of included studies and synthesis of clinical effectiveness data.
Not reported

Not applicable

Not reported for outcomes.

If applicable, describe the population and methods used to elicit preferences

\begin{abstract}
based outcomes
Measurement and val-
\end{abstract}

Estimating resources
and costs

Single study-based economic evaluation: Describe approaches used to estimate resource use associated with the alternative interventions. Describe primary or secondary research methods for valuing each resource item in terms of its unit cost. Describe any adjustments made to approximate to opportunity costs. Not reported y Report the dates of the estimated resource quantities and unit costs. Describe

Currency, price date, and conversion methods for adjusting estimated unit costs to the year of reported costs if nec-

Not reported essary. Describe methods for converting costs into a common currency base and the exchange rate.

\begin{tabular}{llll}
\hline Choice of model & $\begin{array}{l}\text { Describe and give reasons for the specific type of decision-analytical model } \\
\text { used. Providing a figure to show model structure is strongly recommended. }\end{array}$ & Not applicable \\
\hline Assumptions & $\begin{array}{l}\text { Describe all structural or other assumptions underpinning the decision-analyt- } \\
\text { ical model. }\end{array}$ & Not applicable \\
\hline Analytical methods & $\begin{array}{l}\text { Describe all analytical methods supporting the evaluation. This could include } \\
\text { methods for dealing with skewed, missing, or censored data; extrapolation } \\
\text { methods; methods for pooling data; approaches to validate or make adjust- } \\
\text { ments (such as half cycle corrections) to a model; and methods for handling } \\
\text { population heterogeneity and uncertainty. }\end{array}$ & Not applicable \\
\hline
\end{tabular}

\section{Results}

\section{Study parameters}

Report the values, ranges, references, and, if used, probability distributions for all parameters. Report reasons or sources for distributions used to represent uncertainty where appropriate. Providing a table to show the input values is strongly recommended.
Not reported

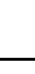

Incremental costs and
outcomes

For each intervention, report mean values for the main categories of estimated costs and outcomes of interest, as well as mean differences between the comparator groups. If applicable, report incremental cost-effectiveness ratios.
Not reported

(1)

\section{Characterising uncer-
tainty \\ Single study-based economic evaluation: Describe the effects of sampling un- certainty for the estimated incremental cost and incremental effectiveness pa- rameters, together with the impact of methodological assumptions (such as discount rate, study perspective).}

Not reported
Model-based economic evaluation: Describe the effects on the results of uncer- tainty for all input parameters, and uncertainty related to the structure of the model and assumptions.

Not applicable
Not reported

\section{If applicable, report differences in costs, outcomes, or cost-effectiveness that can be explained by variations between subgroups of patients with different baseline characteristics or other observed variability in effects that are not re- ducible by more information. \\ Characterising hetero- geneity}


Table 5. CHEERS checklist for methodological quality assessment of economic evaluations (Continued)

\section{Discussion}

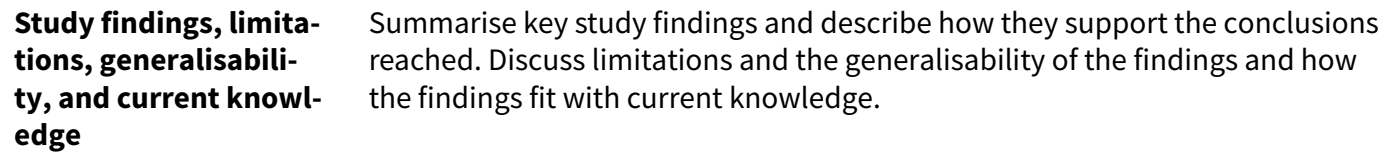

Conflicts of interest

Describe any potential for conflict of interest of study contributors in accordance with journal policy. In the absence of a journal policy, we recommend authors comply with International Committee of Medical Journal Editors recommendations.

\section{Frijling 2002}

Title Identify the study as an economic evaluation or use more specific terms such Not reported
as "cost-effectiveness analysis", and describe the interventions compared.

$\begin{array}{ll}\text { Abstract } & \begin{array}{l}\text { Provide a structured summary of objectives, perspective, setting, methods (in- } \\ \text { cluding study design and inputs), results (including base case and uncertainty }\end{array}\end{array}$
analyses), and conclusions.

\section{Introduction}

\begin{tabular}{|c|c|}
\hline \multirow{2}{*}{$\begin{array}{l}\text { Background and ob- } \\
\text { jectives }\end{array}$} & Provide an explicit statement of the broader context for the study. \\
\hline & $\begin{array}{l}\text { Present the study question and its relevance for health policy or practice deci- } \\
\text { sions. }\end{array}$ \\
\hline
\end{tabular}
sions.

\section{Methods}
Target population and Describe characteristics of the base case population and subgroups analysed, 838 subgroups including why they were chosen.

\begin{tabular}{lll}
\hline Setting and location & $\begin{array}{l}\text { State relevant aspects of the system(s) in which the decision(s) need(s) to be } \\
\text { made. }\end{array}$ & 838 \\
\hline Study perspective & $\begin{array}{l}\text { Describe the perspective of the study and relate this to the costs being evaluat- } \\
\text { ed. }\end{array}$ & Not reported \\
\hline Comparators & $\begin{array}{l}\text { Describe the interventions or strategies being compared and state why they } \\
\text { were chosen. }\end{array}$ & 837 \\
\hline Time horizon & $\begin{array}{l}\text { State the time horizon(s) over which costs and consequences are being evalu- } \\
\text { ated and say why appropriate. }\end{array}$ & Not reported \\
\hline Discount rate & $\begin{array}{l}\text { Report the choice of discount rate(s) used for costs and outcomes and say why } \\
\text { appropriate. }\end{array}$ & Not reported \\
\hline
\end{tabular}


Table 5. CHEERS checklist for methodological quality assessment of economic evaluations (Continued)

Choice of health out- Describe what outcomes were used as the measure(s) of benefit in the evalua- Not reported comes tion and their relevance for the type of analysis performed. $\begin{array}{ll}\begin{array}{l}\text { Measurement of effec- } \\ \text { tiveness }\end{array} & \begin{array}{l}\text { Single study-based estimates: Describe fully the design features of the single } \\ \text { effectiveness study and why the single study was a sufficient source of clinical }\end{array}\end{array}$

Not reported tiveness effectiveness data.

Synthesis-based estimates: Describe fully the methods used for identification Not applicable of included studies and synthesis of clinical effectiveness data.

$\begin{array}{lll}\begin{array}{l}\text { Measurement and val- } \\ \text { uation of preference }\end{array} & \begin{array}{l}\text { If applicable, describe the population and methods used to elicit preferences } \\ \text { for outcomes. }\end{array} & \text { Not reported } \\ \end{array}$
based outcomes

\author{
Estimating resources \\ and costs
}

Single study-based economic evaluation: Describe approaches used to estimate resource use associated with the alternative interventions. Describe priof its unit cost. Describe any adjustments made to approximate to opportunity costs. mary or secondary research methods for valuing each resource item in terms

Not reported

Report the dates of the estimated resource quantities and unit costs. Describe methods for adjusting estimated unit costs to the year of reported costs if necessary. Describe methods for converting costs into a common currency base and the exchange rate.

\section{Currency, price date, and conversion}

\section{Not reported}

d

\begin{tabular}{lll}
\hline Choice of model & $\begin{array}{l}\text { Describe and give reasons for the specific type of decision-analytical model } \\
\text { used. Providing a figure to show model structure is strongly recommended. }\end{array}$ & Not applicable \\
\hline Assumptions & $\begin{array}{l}\text { Describe all structural or other assumptions underpinning the decision-analyt- } \\
\text { ical model. }\end{array}$ & Not applicable \\
\hline Analytical methods & $\begin{array}{l}\text { Describe all analytical methods supporting the evaluation. This could include } \\
\text { methods for dealing with skewed, missing, or censored data; extrapolation } \\
\text { methods; methods for pooling data; approaches to validate or make adjust- } \\
\text { ments (such as half cycle corrections) to a model; and methods for handling } \\
\text { population heterogeneity and uncertainty. }\end{array}$ & Not reported \\
\hline
\end{tabular}

\section{Results}

\begin{tabular}{ll}
\hline Study parameters & $\begin{array}{l}\text { Report the values, ranges, references, and, if used, probability distributions for } \\
\text { all parameters. Report reasons or sources for distributions used to represent } \\
\text { uncertainty where appropriate. Providing a table to show the input values is } \\
\text { strongly recommended. }\end{array}$ \\
\hline
\end{tabular}

$\begin{array}{ll}\begin{array}{l}\text { Incremental costs and } \\ \text { outcomes }\end{array} & \begin{array}{l}\text { For each intervention, report mean values for the main categories of estimated } \\ \text { costs and outcomes of interest, as well as mean differences between the com- } \\ \text { parator groups. If applicable, report incremental cost-effectiveness ratios. }\end{array}\end{array}$

Characterising uncer-
tainty

Single study-based economic evaluation: Describe the effects of sampling uncertainty for the estimated incremental cost and incremental effectiveness parameters, together with the impact of methodological assumptions (such as discount rate, study perspective).

Not reported

Model-based economic evaluation: Describe the effects on the results of uncertainty for all input parameters, and uncertainty related to the structure of the model and assumptions. Not applicable

Not reported

Not reported

Not applicable


Table 5. CHEERS checklist for methodological quality assessment of economic evaluations (Continued)

Characterising hetero- If applicable, report differences in costs, outcomes, or cost-effectiveness that geneity

can be explained by variations between subgroups of patients with different baseline characteristics or other observed variability in effects that are not reducible by more information.

\section{Discussion}

Study findings, limita- Summarise key study findings and describe how they support the conclusions

841

tions, generalisabilireached. Discuss limitations and the generalisability of the findings and how

Not applicable

ty, and current knowl- the findings fit with current knowledge.

edge

\section{Other}

Source of funding Describe how the study was funded and the role of the funder in the identification, design, conduct, and reporting of the analysis. Describe other non-monetary sources of support.

Conflicts of interest

Describe any potential for conflict of interest of study contributors in accordance with journal policy. In the absence of a journal policy, we recommend Not reported authors comply with International Committee of Medical Journal Editors recommendations.

\section{Krein 2004}

\begin{tabular}{ll}
\hline Title & Identify the study as an economic evaluation or use more specific terms such \\
as "cost-effectiveness analysis", and describe the interventions compared.
\end{tabular}

\begin{tabular}{lll}
\hline Abstract & $\begin{array}{l}\text { Provide a structured summary of objectives, perspective, setting, methods (in- } \\
\text { cluding study design and inputs), results (including base case and uncertainty } \\
\text { analyses), and conclusions. }\end{array}$ & Not reported \\
\hline Introduction & & 732 \\
\hline $\begin{array}{l}\text { Background and ob- } \\
\text { jectives }\end{array}$ & \begin{tabular}{l} 
Provide an explicit statement of the broader context for the study. \\
\cline { 2 - 4 } \\
Present the study question and its relevance for health policy or practice deci-
\end{tabular} & 732 \\
\hline
\end{tabular}

\section{Methods}
Target population and Describe characteristics of the base case population and subgroups analysed, 733 subgroups including why they were chosen.

\begin{tabular}{ll}
\hline Setting and location & $\begin{array}{l}\text { State relevant aspects of the system(s) in which the decision(s) need(s) to be } \\
\text { made. }\end{array}$
\end{tabular}

\begin{tabular}{ll}
\hline Study perspective & Describe the perspective of the study and relate this to the costs being evaluat- Not reported \\
ed.
\end{tabular}

\begin{tabular}{lll}
\hline Comparators & $\begin{array}{l}\text { Describe the interventions or strategies being compared and state why they } \\
\text { were chosen. }\end{array}$ & 733
\end{tabular}

\section{Time horizon}

State the time horizon(s) over which costs and consequences are being evaluated and say why appropriate. 
Table 5. CHEERS checklist for methodological quality assessment of economic evaluations (Continued)
Discount rate
Report the choice of discount rate(s) used for costs and outcomes and say why
Not reported appropriate.

\section{Choice of health out- comes}

Measurement of effectiveness
Describe what outcomes were used as the measure(s) of benefit in the evaluation and their relevance for the type of analysis performed.
Single study-based estimates: Describe fully the design features of the single effectiveness study and why the single study was a sufficient source of clinical effectiveness data.
Not reported

Not reported

Synthesis-based estimates: Describe fully the methods used for identification of included studies and synthesis of clinical effectiveness data.

\section{Measurement and val- If applicable, describe the population and methods used to elicit preferences Not reported uation of preference for outcomes.}

based outcomes

\section{Estimating resources and costs}

Single study-based economic evaluation: Describe approaches used to estimate resource use associated with the alternative interventions. Describe primary or secondary research methods for valuing each resource item in terms of its unit cost. Describe any adjustments made to approximate to opportunity

\section{Not reported}

Report the dates of the estimated resource quantities and unit costs. Describe methods for adjusting estimated unit costs to the year of reported costs if necessary. Describe methods for converting costs into a common currency base and the exchange rate.
Not reported

Not reported

\section{(a)}


Table 5. CHEERS checklist for methodological quality assessment of economic evaluations (Continued)

Model-based economic evaluation: Describe the effects on the results of uncer- Not applicable tainty for all input parameters, and uncertainty related to the structure of the model and assumptions.

Characterising hetero- If applicable, report differences in costs, outcomes, or cost-effectiveness that geneity can be explained by variations between subgroups of patients with different baseline characteristics or other observed variability in effects that are not reducible by more information.

\section{Discussion}
Study findings, limita- Summarise key study findings and describe how they support the conclusions tions, generalisabili- reached. Discuss limitations and the generalisability of the findings and how ty, and current knowl- the findings fit with current knowledge. edge

\section{Other}

Source of funding Describe how the study was funded and the role of the funder in the identification, design, conduct, and reporting of the analysis. Describe other non-monetary sources of support.

\section{Conflicts of interest Describe any potential for conflict of interest of study contributors in accor- dance with journal policy. In the absence of a journal policy, we recommend authors comply with International Committee of Medical Journal Editors rec- ommendations.}

\section{Litaker 2003}

\begin{tabular}{lll}
\hline Title & $\begin{array}{l}\text { Identify the study as an economic evaluation or use more specific terms such } \\
\text { as "cost-effectiveness analysis", and describe the interventions compared. }\end{array}$ & front page \\
\hline Abstract & $\begin{array}{l}\text { Provide a structured summary of objectives, perspective, setting, methods (in- } \\
\text { cluding study design and inputs), results (including base case and uncertainty } \\
\text { analyses), and conclusions. }\end{array}$ & Not reported \\
\hline Introduction & & 224 \\
\hline $\begin{array}{l}\text { Background and ob- } \\
\text { jectives }\end{array}$ & Provide an explicit statement of the broader context for the study. & 224 \\
\cline { 2 - 4 } & $\begin{array}{l}\text { Present the study question and its relevance for health policy or practice deci- } \\
\text { sions. }\end{array}$ & \\
\hline
\end{tabular}

\section{Methods}
Target population and Describe characteristics of the base case population and subgroups analysed, 225 subgroups including why they were chosen.

\begin{tabular}{lll}
\hline Setting and location & $\begin{array}{l}\text { State relevant aspects of the system(s) in which the decision(s) need(s) to be } \\
\text { made. }\end{array}$ & 225
\end{tabular}
Study perspective Describe the perspective of the study and relate this to the costs being evaluat- Not reported ed.


Table 5. CHEERS checklist for methodological quality assessment of economic evaluations (Continued)
Comparators
Describe the interventions or strategies being compared and state why they
226 were chosen.

\begin{tabular}{lll}
\hline Time horizon & $\begin{array}{l}\text { State the time horizon(s) over which costs and consequences are being evalu- } \\
\text { ated and say why appropriate. }\end{array}$ & Not reported \\
\hline Discount rate & $\begin{array}{l}\text { Report the choice of discount rate(s) used for costs and outcomes and say why } \\
\text { appropriate. }\end{array}$ & Not reported
\end{tabular}

$\begin{array}{lll}\begin{array}{l}\text { Choice of health out- } \\ \text { comes }\end{array} & \begin{array}{l}\text { Describe what outcomes were used as the measure(s) of benefit in the evalua- } \\ \text { tion and their relevance for the type of analysis performed. }\end{array}\end{array}$
comes

Single study-based estimates: Describe fully the design features of the single Not reported effectiveness study and why the single study was a sufficient source of clinical effectiveness data.
Measurement of effectiveness
Synthesis-based estimates: Describe fully the methods used for identification of included studies and synthesis of clinical effectiveness data.
Not reported

\section{6}

If applicable,
for outcomes. uation of preference based outcomes

\section{Estimating resources} and costs

Single study-based economic evaluation: Describe approaches used to esti-

\section{Currency, price date, and conversion}

Report the dates of the estimated resource quantities and unit costs. Describe

\begin{tabular}{lll}
\hline Choice of model & $\begin{array}{l}\text { Describe and give reasons for the specific type of decision-analytical model } \\
\text { used. Providing a figure to show model structure is strongly recommended. }\end{array} \quad$ Not applicable
\end{tabular}
mate resource use associated with the alternative interventions. Describe primary or secondary research methods for valuing each resource item in terms of its unit cost. Describe any adjustments made to approximate to opportunity costs. methods for adjusting estimated unit costs to the year of reported costs if necessary. Describe methods for converting costs into a common currency base and the exchange rate.

Not reported

Not reported

Not applicable ical model.

\begin{tabular}{ll}
\hline Analytical methods & Describe all analytical methods supporting the evaluation. This could include \\
methods for dealing with skewed, missing, or censored data; extrapolation \\
methods; methods for pooling data; approaches to validate or make adjust- \\
ments (such as half cycle corrections) to a model; and methods for handling \\
population heterogeneity and uncertainty.
\end{tabular}

Not applicable population heterogeneity and uncertainty.

\section{Results}

$\begin{array}{ll}\text { Study parameters } & \text { Report the values, ranges, references, and, if used, probability distributions for Not reported } \\ \text { all parameters. Report reasons or sources for distributions used to represent } \\ \text { uncertainty where appropriate. Providing a table to show the input values is } \\ \text { strongly recommended. }\end{array}$

\section{Incremental costs and outcomes}

For each intervention, report mean values for the main categories of estimated costs and outcomes of interest, as well as mean differences between the comparator groups. If applicable, report incremental cost-effectiveness ratios.
Not reported

Notreported 
Table 5. CHEERS checklist for methodological quality assessment of economic evaluations (Continued) Characterising uncer-
tainty
Single study-based economic evaluation: Describe the effects of sampling uncertainty for the estimated incremental cost and incremental effectiveness parameters, together with the impact of methodological assumptions (such as discount rate, study perspective).

\section{Not reported}

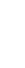

Model-based economic evaluation: Describe the effects on the results of uncer-
tainty for all input parameters, and uncertainty related to the structure of the

Not applicable model and assumptions.

If applicable, report differences in costs, outcomes, or cost-effectiveness that can be explained by variations between subgroups of patients with different baseline characteristics or other observed variability in effects that are not reducible by more information.

\section{2}

geneity

Characterising heterogeneity

\section{Discussion}
Study findings, limita- tions, generalisabili- ty, and current knowl- edge
Summarise key study findings and describe how they support the conclusions reached. Discuss limitations and the generalisability of the findings and how the findings fit with current knowledge.

234

Other

\begin{tabular}{ll}
\hline Source of funding & $\begin{array}{l}\text { Describe how the study was funded and the role of the funder in the identifica- } \\
\text { tion, design, conduct, and reporting of the analysis. Describe other non-mone- } \\
\text { tary sources of support. }\end{array}$
\end{tabular}

\begin{tabular}{ll}
\hline Conflicts of interest & Describe any potential for conflict of interest of study contributors in accor- \\
& dance with journal policy. In the absence of a journal policy, we recommend \\
& authors comply with International Committee of Medical Journal Editors rec- \\
& ommendations.
\end{tabular}

Not reported authors comply with International Committee of Medical Journal Editors recommendations.

\section{McCall 2011}

\begin{tabular}{lll}
\hline Title & $\begin{array}{l}\text { Identify the study as an economic evaluation or use more specific terms such } \\
\text { as "cost-effectiveness analysis", and describe the interventions compared. }\end{array}$ & Not reported \\
\hline Abstract & $\begin{array}{l}\text { Provide a structured summary of objectives, perspective, setting, methods (in- } \\
\text { cluding study design and inputs), results (including base case and uncertainty } \\
\text { analyses), and conclusions. }\end{array}$ & Not reported \\
\hline Introduction & Provide an explicit statement of the broader context for the study. & 1705 \\
\hline $\begin{array}{l}\text { Background and ob- } \\
\text { jectives }\end{array}$ & $\begin{array}{l}\text { Present the study question and its relevance for health policy or practice deci- } \\
\text { sions. }\end{array}$ & 1706 \\
\hline
\end{tabular}

\section{Methods}

$\begin{array}{lll}\begin{array}{l}\text { Target population and } \\ \text { subgroups }\end{array} & \begin{array}{l}\text { Describe characteristics of the base case population and subgroups analysed, } \\ \text { including why they were chosen. }\end{array} & 1708\end{array}$

\begin{tabular}{lll}
\hline Setting and location & $\begin{array}{l}\text { State relevant aspects of the system(s) in which the decision(s) need(s) to be } \\
\text { made. }\end{array}$ & 1705
\end{tabular}


Table 5. CHEERS checklist for methodological quality assessment of economic evaluations (Continued)
Study perspective
Describe the perspective of the study and relate this to the costs being evaluat- Not reported ed.

\begin{tabular}{ll}
\hline Comparators & $\begin{array}{l}\text { Describe the interventions or strategies being compared and state why they } \\
\text { were chosen. }\end{array}$ \\
\hline Time horizon & $\begin{array}{l}\text { State the time horizon(s) over which costs and consequences are being evalu- } \\
\text { ated and say why appropriate. }\end{array}$ \\
\hline Discount rate & $\begin{array}{l}\text { Report the choice of discount rate(s) used for costs and outcomes and say why } \\
\text { appropriate. }\end{array}$
\end{tabular}

\begin{tabular}{ll}
\hline $\begin{array}{l}\text { Choice of health out- } \\
\text { comes }\end{array}$ & $\begin{array}{l}\text { Describe what outcomes were used as the measure(s) of benefit in the evalua- } \\
\text { tion and their relevance for the type of analysis performed. }\end{array}$
\end{tabular}

\begin{tabular}{lll}
\hline $\begin{array}{l}\text { Measurement of effec- } \\
\text { tiveness }\end{array}$ & $\begin{array}{l}\text { Single study-based estimates: Describe fully the design features of the single } \\
\text { effectiveness study and why the single study was a sufficient source of clinical }\end{array} \quad$ Not reported
\end{tabular}
effectiveness data. Synthesis-based estimates: Describe fully the methods used for identification of included studies and synthesis of clinical effectiveness data.

\begin{tabular}{lll}
\hline $\begin{array}{l}\text { Measurement and val- } \\
\text { uation of preference }\end{array}$ & for autcomes.
\end{tabular}
uation of preference based outcomes

\section{Estimating resources and costs}

Single study-based economic evaluation: Describe approaches used to estimate resource use associated with the alternative interventions. Describe primary or secondary research methods for valuing each resource item in terms of its unit cost. Describe any adjustments made to approximate to opportunity costs. Not applicable

Not reported

\author{
Notreported
}

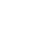
Report the dates of the estimated resource quantities and unit costs. Describe

$\begin{array}{ll}\begin{array}{l}\text { Currency, price date, } \\ \text { and conversion }\end{array} & \begin{array}{l}\text { Report the dates of the estimated resource quantities and unit costs. Describe } \\ \text { methods for adjusting estimated unit costs to the year of reported costs if nec- } \\ \text { essary. Describe methods for converting costs into a common currency base } \\ \text { and the exchange rate. }\end{array}\end{array}$

\begin{tabular}{lll}
\hline Choice of model & Describe and give reasons for the specific type of decision-analytical model \\
used. Providing a figure to show model structure is strongly recommended.
\end{tabular}

\begin{tabular}{lll}
\hline Assumptions & $\begin{array}{l}\text { Describe all structural or other assumptions underpinning the decision-analyt- } \\
\text { ical model. }\end{array}$ & Not applicable \\
\hline Analytical methods & $\begin{array}{l}\text { Describe all analytical methods supporting the evaluation. This could include } \\
\text { methods for dealing with skewed, missing, or censored data; extrapolation } \\
\text { methods; methods for pooling data; approaches to validate or make adjust- } \\
\text { ments (such as half cycle corrections) to a model; and methods for handling } \\
\text { population heterogeneity and uncertainty. }\end{array}$ & Not applicable \\
\hline
\end{tabular}

\section{Results}

\begin{tabular}{ll}
\hline Study parameters & Report the values, ranges, references, and, if used, probability distributions for Not reported \\
all parameters. Report reasons or sources for distributions used to represent \\
uncertainty where appropriate. Providing a table to show the input values is \\
strongly recommended.
\end{tabular}


Table 5. CHEERS checklist for methodological quality assessment of economic evaluations (Continued)

$\begin{array}{ll}\begin{array}{l}\text { Incremental costs and } \\ \text { outcomes }\end{array} & \begin{array}{l}\text { For each intervention, report mean values for the main categories of estimated } \\ \text { costs and outcomes of interest, as well as mean differences between the com- } \\ \text { parator groups. If applicable, report incremental cost-effectiveness ratios. }\end{array}\end{array}$

\begin{tabular}{ll}
\hline Characterising uncer- & Single study-based economic evaluation: Describe the effects of sampling un- \\
tainty & $\begin{array}{l}\text { certainty for the estimated incremental cost and incremental effectiveness pa- } \\
\text { rameters, together with the impact of methodological assumptions (such as } \\
\text { discount rate, study perspective). }\end{array}$
\end{tabular}

Model-based economic evaluation: Describe the effects on the results of uncertainty for all input parameters, and uncertainty related to the structure of the Not applicable model and assumptions

$\begin{array}{ll}\text { Characterising hetero- } & \begin{array}{l}\text { If applicable, report differences in costs, outcomes, or cost-effectiveness that } \\ \text { geneity }\end{array} \\ \begin{array}{l}\text { can be explained by variations between subgroups of patients with different } \\ \text { baseline characteristics or other observed variability in effects that are not re- } \\ \text { ducible by more information. }\end{array}\end{array}$

Not applicable ducible by more information.

\section{Discussion}

Study findings, limita- Summarise key study findings and describe how they support the conclusions reached. Discuss limitations and the generalisability of the findings and how ty, and current knowl- the findings fit with current knowledge. edge

\section{Other}

Source of funding Describe how the study was funded and the role of the funder in the identification, design, conduct, and reporting of the analysis. Describe other non-monetary sources of support.

\section{Conflicts of interest}

Describe any potential for conflict of interest of study contributors in accordance with journal policy. In the absence of a journal policy, we recommend Not reported authors comply with International Committee of Medical Journal Editors recommendations.

\section{Piette 2001}

\begin{tabular}{lll}
\hline Title & $\begin{array}{l}\text { Identify the study as an economic evaluation or use more specific terms such } \\
\text { as "cost-effectiveness analysis", and describe the interventions compared. }\end{array}$ & Not reported \\
\hline Abstract & $\begin{array}{l}\text { Provide a structured summary of objectives, perspective, setting, methods (in- } \\
\text { cluding study design and inputs), results (including base case and uncertainty } \\
\text { analyses), and conclusions. }\end{array}$ & Not reported \\
\hline Introduction & Provide an explicit statement of the broader context for the study. & 202 - 203 \\
\hline $\begin{array}{l}\text { Background and ob- } \\
\text { jectives }\end{array}$ & $\begin{array}{l}\text { Present the study question and its relevance for health policy or practice deci- } \\
\text { sions. }\end{array}$ & Not reported \\
\hline
\end{tabular}

\section{Methods}


Table 5. CHEERS checklist for methodological quality assessment of economic evaluations (Continued)
Target population and
Describe characteristics of the base case population and subgroups analysed,
204 subgroups including why they were chosen.

\begin{tabular}{ll}
\hline Setting and location & $\begin{array}{l}\text { State relevant aspects of the system(s) in which the decision(s) need(s) to be } \\
\text { made. }\end{array}$
\end{tabular}

\begin{tabular}{lll}
\hline Study perspective & $\begin{array}{l}\text { Describe the perspective of the study and relate this to the costs being evaluat- } \\
\text { ed. }\end{array}$ & Not reported \\
\hline Comparators & $\begin{array}{l}\text { Describe the interventions or strategies being compared and state why they } \\
\text { were chosen. }\end{array}$ & 177
\end{tabular}

\begin{tabular}{ll}
\hline Time horizon & $\begin{array}{l}\text { State the time horizon(s) over which costs and consequences are being evalu- Not reported } \\
\text { ated and say why appropriate. }\end{array}$ \\
\hline
\end{tabular}

\begin{tabular}{lll}
\hline Discount rate & $\begin{array}{l}\text { Report the choice of discount rate(s) used for costs and outcomes and say why } \\
\text { appropriate. }\end{array}$ & Not reported \\
\hline $\begin{array}{l}\text { Choice of health out- } \\
\text { comes }\end{array}$ & $\begin{array}{l}\text { Describe what outcomes were used as the measure(s) of benefit in the evalua- } \\
\text { tion and their relevance for the type of analysis performed. }\end{array}$ & Not reported \\
\hline
\end{tabular}

$\begin{array}{ll}\text { Measurement of effec- } & \text { Single study-based estimates: Describe fully the design features of the single } \\ \text { tiveness } & \text { effectiveness study and why the single study was a sufficient source of clinical }\end{array}$
tiveness effectiveness study and why the single study was a sufficient source of clinical effectiveness data.

Synthesis-based estimates: Describe fully the methods used for identification Not applicable of included studies and synthesis of clinical effectiveness data.

$\begin{array}{ll}\begin{array}{l}\text { Measurement and val- } \\ \text { uation of preference }\end{array} & \begin{array}{l}\text { If applicable, describe the population and methods used to elicit preferences } \\ \text { for outcomes. }\end{array}\end{array}$
based outcomes
Estimating resources Single study-based economic evaluation: Describe approaches used to esti- and costs mate resource use associated with the alternative interventions. Describe pri- mary or secondary research methods for valuing each resource item in terms of its unit cost. Describe any adjustments made to approximate to opportunity costs.

Not reported Report the dates of the estimated resource quantities and unit costs. Describe methods for adjusting estimated unit costs to the year of reported costs if necNot reported

Currency, price date, and conversion essary. Describe methods for converting costs into a common currency base and the exchange rate.

\begin{tabular}{llll}
\hline Choice of model & $\begin{array}{l}\text { Describe and give reasons for the specific type of decision-analytical model } \\
\text { used. Providing a figure to show model structure is strongly recommended. }\end{array}$ & Not applicable \\
\hline Assumptions & $\begin{array}{l}\text { Describe all structural or other assumptions underpinning the decision-analyt- } \\
\text { ical model. }\end{array}$ & Not applicable \\
\hline Analytical methods & $\begin{array}{l}\text { Describe all analytical methods supporting the evaluation. This could include } \\
\text { methods for dealing with skewed, missing, or censored data; extrapolation } \\
\text { methods; methods for pooling data; approaches to validate or make adjust- } \\
\text { ments (such as half cycle corrections) to a model; and methods for handling } \\
\text { population heterogeneity and uncertainty. }\end{array}$ & Not applicable \\
\hline
\end{tabular}

\section{Results}


Table 5. CHEERS checklist for methodological quality assessment of economic evaluations (Continued)

Study parameters

\section{Characterising uncer- tainty}

Report the values, ranges, references, and, if used, probability distributions for Not reported all parameters. Report reasons or sources for distributions used to represent uncertainty where appropriate. Providing a table to show the input values is strongly recommended.

\section{outcomes \\ Incremental costs and} For each intervention, report mean values for the main categories of estimated costs and outcomes of interest, as well as mean differences between the comparator groups. If applicable, report incremental cost-effectiveness ratios.
Not reported

Single study-based economic evaluation: Describe the effects of sampling uncertainty for the estimated incremental cost and incremental effectiveness parameters, together with the impact of methodological assumptions (such as discount rate, study perspective).

Model-based economic evaluation: Describe the effects on the results of uncertainty for all input parameters, and uncertainty related to the structure of the Not applicable model and assumptions.
Not reported

\begin{tabular}{ll}
\hline $\begin{array}{l}\text { Characterising hetero- } \\
\text { geneity }\end{array}$ & $\begin{array}{l}\text { If applicable, report differences in costs, outcomes, or cost-effectiveness that } \\
\text { can be explained by variations between subgroups of patients with different } \\
\text { baseline characteristics or other observed variability in effects that are not re- } \\
\text { ducible by more information. }\end{array}$
\end{tabular}

Not reported

\section{Discussion}
Study findings, limita- tions, generalisabili- ty, and current knowl- edge
Summarise key study findings and describe how they support the conclusions reached. Discuss limitations and the generalisability of the findings and how the findings fit with current knowledge.

\section{Other}

Source of funding $\begin{aligned} & \text { Describe how the study was funded and the role of the funder in the identifica- } \\ & \text { tion, design, conduct, and reporting of the analysis. Describe other non-mone- } \\ & \text { tary sources of support. }\end{aligned}$

$\begin{array}{ll}\text { Conflicts of interest } & \begin{array}{l}\text { Describe any potential for conflict of interest of study contributors in accor- } \\ \text { dance with journal policy. In the absence of a journal policy, we recommend } \\ \text { authors comply with International Committee of Medical Journal Editors rec- } \\ \text { ommendations. }\end{array}\end{array}$
authors comply with International Committee of Medical Journal Editors recommendations.

\begin{tabular}{lll}
\hline Pizzi $\mathbf{2 0 1 5}$ & & $\begin{array}{l}\text { Identify the study as an economic evaluation or use more specific terms such } \\
\text { as "cost-effectiveness analysis", and describe the interventions compared. }\end{array}$ \\
\hline Title & $\begin{array}{l}\text { Provide a structured summary of objectives, perspective, setting, methods (in- front page } \\
\text { cluding study design and inputs), results (including base case and uncertainty } \\
\text { analyses), and conclusions. }\end{array}$ \\
\hline Abstract & & 254 \\
\hline Introduction & Provide an explicit statement of the broader context for the study. \\
\hline $\begin{array}{l}\text { Background and ob- } \\
\text { jectives }\end{array}$ &
\end{tabular}


Table 5. CHEERS checklist for methodological quality assessment of economic evaluations (Continued)

Present the study question and its relevance for health policy or practice deci- 254 sions.

\begin{tabular}{lll}
\hline Methods & & \\
\hline $\begin{array}{l}\text { Target population and } \\
\text { subgroups }\end{array}$ & $\begin{array}{l}\text { Describe characteristics of the base case population and subgroups analysed, } \\
\text { including why they were chosen. }\end{array}$ & 254 \\
\hline Setting and location & $\begin{array}{l}\text { State relevant aspects of the system(s) in which the decision(s) need(s) to be } \\
\text { made. }\end{array}$ & 254 \\
\hline Study perspective & $\begin{array}{l}\text { Describe the perspective of the study and relate this to the costs being evaluat- } \\
\text { ed. }\end{array}$ & 255
\end{tabular}

\begin{tabular}{lll}
\hline Comparators & $\begin{array}{l}\text { Describe the interventions or strategies being compared and state why they } \\
\text { were chosen. }\end{array}$ & 254
\end{tabular}

\begin{tabular}{lll}
\hline Time horizon & State the time horizon(s) over which costs and consequences are being evalu- & 256 \\
ated and say why appropriate.
\end{tabular}

\begin{tabular}{ll}
\hline Discount rate & $\begin{array}{l}\text { Report the choice of discount rate(s) used for costs and outcomes and say why } 256 \\
\text { appropriate. }\end{array}$
\end{tabular}

\begin{tabular}{lll}
\hline $\begin{array}{l}\text { Choice of health out- } \\
\text { comes }\end{array}$ & $\begin{array}{l}\text { Describe what outcomes were used as the measure(s) of benefit in the evalua- } \\
\text { tion and their relevance for the type of analysis performed. }\end{array}$ & 255 \\
\hline
\end{tabular}

\begin{tabular}{lll}
\hline $\begin{array}{l}\text { Measurement of effec- } \\
\text { tiveness }\end{array}$ & $\begin{array}{l}\text { Single study-based estimates: Describe fully the design features of the single } \\
\text { effectiveness study and why the single study was a sufficient source of clinical } \\
\text { effectiveness data. }\end{array}$ & $254-255$
\end{tabular}

Synthesis-based estimates: Describe fully the methods used for identification Not reported of included studies and synthesis of clinical effectiveness data.

$\begin{array}{ll}\begin{array}{l}\text { Measurement and val- } \\ \text { uation of preference } \\ \text { based outcomes }\end{array} & \begin{array}{l}\text { If applicable, describe the population and methods used to elicit preferences } \\ \text { for outcomes. }\end{array}\end{array}$
based outcomes

$\begin{array}{ll}\text { Estimating resources } & \text { Single study-based economic evaluation: Describe approaches used to esti- } \\ \text { and costs } & \text { mate resource use associated with the alternative interventions. Describe pri- } \\ & \text { mary or secondary research methods for valuing each resource item in terms } \\ \text { of its unit cost. Describe any adjustments made to approximate to opportunity } \\ \text { costs. }\end{array}$

\begin{tabular}{ll}
\hline $\begin{array}{l}\text { Currency, price date, } \\
\text { and conversion }\end{array}$ & $\begin{array}{l}\text { Report the dates of the estimated resource quantities and unit costs. Describe } \\
\text { methods for adjusting estimated unit costs to the year of reported costs if nec- } \\
\text { essary. Describe methods for converting costs into a common currency base } \\
\text { and the exchange rate. }\end{array}$
\end{tabular}

\begin{tabular}{lll}
\hline Choice of model & Describe and give reasons for the specific type of decision-analytical model & 256 \\
used. Providing a figure to show model structure is strongly recommended.
\end{tabular}

\begin{tabular}{ll}
\hline Assumptions & $\begin{array}{l}\text { Describe all structural or other assumptions underpinning the decision-analyt- } \\
\text { ical model. }\end{array}$
\end{tabular}

\begin{tabular}{ll}
\hline Analytical methods & Describe all analytical methods supporting the evaluation. This could include \\
methods for dealing with skewed, missing, or censored data; extrapolation \\
methods; methods for pooling data; approaches to validate or make adjust-
\end{tabular}


Table 5. CHEERS checklist for methodological quality assessment of economic evaluations (Continued)

ments (such as half cycle corrections) to a model; and methods for handling population heterogeneity and uncertainty.

\section{Results}

Study parameters Report the values, ranges, references, and, if used, probability distributions for all parameters. Report reasons or sources for distributions used to represent uncertainty where appropriate. Providing a table to show the input values is strongly recommended.

\section{Incremental costs and outcomes}

\section{Characterising uncer- tainty}

For each intervention, report mean values for the main categories of estimated costs and outcomes of interest, as well as mean differences between the comparator groups. If applicable, report incremental cost-effectiveness ratios.

\section{0}

Single study-based economic evaluation: Describe the effects of sampling un$258-260$ certainty for the estimated incremental cost and incremental effectiveness parameters, together with the impact of methodological assumptions (such as discount rate, study perspective).

Model-based economic evaluation: Describe the effects on the results of uncertainty for all input parameters, and uncertainty related to the structure of the model and assumptions.

\section{Characterising hetero- geneity}

If applicable, report differences in costs, outcomes, or cost-effectiveness that can be explained by variations between subgroups of patients with different baseline characteristics or other observed variability in effects that are not reducible by more information.

\section{Discussion}

Study findings, limitations, generalisability, and current knowledge
Summarise key study findings and describe how they support the conclusions reached. Discuss limitations and the generalisability of the findings and how the findings fit with current knowledge.

\section{Other}

Source of funding Describe how the study was funded and the role of the funder in the identification, design, conduct, and reporting of the analysis. Describe other non-monetary sources of support.

Conflicts of interest Describe any potential for conflict of interest of study contributors in accordance with journal policy. In the absence of a journal policy, we recommend authors comply with International Committee of Medical Journal Editors recommendations.

\section{Prezio 2014}

\begin{tabular}{lll}
\hline Title & $\begin{array}{l}\text { Identify the study as an economic evaluation or use more specific terms such } \\
\text { as "cost-effectiveness analysis", and describe the interventions compared. }\end{array}$ & 771 \\
\hline Abstract & $\begin{array}{l}\text { Provide a structured summary of objectives, perspective, setting, methods (in- } \\
\text { cluding study design and inputs), results (including base case and uncertainty } \\
\text { analyses), and conclusions. }\end{array}$ & 771 \\
&
\end{tabular}

\section{Introduction}


Table 5. CHEERS checklist for methodological quality assessment of economic evaluations (Continued)

Background and ob- Provide an explicit statement of the broader context for the study.

772 jectives

Present the study question and its relevance for health policy or practice deci- $\quad 772$ sions.

\section{Methods} $\begin{array}{lll}\begin{array}{l}\text { Target population and } \\ \text { subgroups }\end{array} & \begin{array}{l}\text { Describe characteristics of the base case population and subgroups analysed, } \\ \text { including why they were chosen. }\end{array} & 772\end{array}$

Setting and location State relevant aspects of the system(s) in which the decision(s) need(s) to be 772
made.

Study perspective Describe the perspective of the study and relate this to the costs being evaluat- 772
ed.

Comparators Describe the interventions or strategies being compared and state why they 772
were chosen.

\begin{tabular}{lll}
\hline Time horizon & $\begin{array}{l}\text { State the time horizon(s) over which costs and consequences are being evalu- } \\
\text { ated and say why appropriate. }\end{array}$ & 772 \\
\hline Discount rate & $\begin{array}{l}\text { Report the choice of discount rate(s) used for costs and outcomes and say why } \\
\text { appropriate. }\end{array}$ & 772 \\
\hline
\end{tabular}

\begin{tabular}{lll}
\hline $\begin{array}{l}\text { Choice of health out- } \\
\text { comes }\end{array}$ & $\begin{array}{l}\text { Describe what outcomes were used as the measure(s) of benefit in the evalua- } \\
\text { tion and their relevance for the type of analysis performed. }\end{array}$ & 774 \\
\hline
\end{tabular}

\begin{tabular}{llll}
\hline $\begin{array}{l}\text { Measurement of effec- } \\
\text { tiveness }\end{array}$ & $\begin{array}{l}\text { Single study-based estimates: Describe fully the design features of the single } \\
\text { effectiveness study and why the single study was a sufficient source of clinical }\end{array}$ & 772
\end{tabular}
tiveness effectiveness data.

Synthesis-based estimates: Describe fully the methods used for identification Not reported of included studies and synthesis of clinical effectiveness data.

\begin{tabular}{|c|c|c|}
\hline $\begin{array}{l}\text { Measurement and val- } \\
\text { uation of preference }\end{array}$ & $\begin{array}{l}\text { If applicable, describe the population and methods used to elicit preferences } \\
\text { for outcomes. }\end{array}$ & Not applicab \\
\hline
\end{tabular}
uation of preference
based outcomes

\begin{tabular}{ll}
\hline Estimating resources & Single study-based economic evaluation: Describe approaches used to esti- \\
and costs & mate resource use associated with the alternative interventions. Describe pri- \\
& mary or secondary research methods for valuing each resource item in terms \\
of its unit cost. Describe any adjustments made to approximate to opportunity \\
costs.
\end{tabular}

\begin{tabular}{ll}
\hline $\begin{array}{l}\text { Currency, price date, } \\
\text { and conversion }\end{array}$ & $\begin{array}{l}\text { Report the dates of the estimated resource quantities and unit costs. Describe } \\
\text { methods for adjusting estimated unit costs to the year of reported costs if nec- } \\
\text { essary. Describe methods for converting costs into a common currency base } \\
\text { and the exchange rate. }\end{array}$
\end{tabular}

\begin{tabular}{ll}
\hline Choice of model & $\begin{array}{l}\text { Describe and give reasons for the specific type of decision-analytical model } \\
\text { used. Providing a figure to show model structure is strongly recommended. }\end{array}$
\end{tabular}

\begin{tabular}{lll}
\hline Assumptions & $\begin{array}{l}\text { Describe all structural or other assumptions underpinning the decision-analyt- } \\
\text { ical model. }\end{array}$ & $772-77$ \\
\hline Analytical methods & $\begin{array}{l}\text { Describe all analytical methods supporting the evaluation. This could include } \\
\text { methods for dealing with skewed, missing, or censored data; extrapolation }\end{array}$ & 774 \\
&
\end{tabular}


Table 5. CHEERS checklist for methodological quality assessment of economic evaluations (Continued)

methods; methods for pooling data; approaches to validate or make adjustments (such as half cycle corrections) to a model; and methods for handling population heterogeneity and uncertainty.

\begin{tabular}{ll}
\hline Results & \\
\hline Study parameters & $\begin{array}{l}\text { Report the values, ranges, references, and, if used, probability distributions for } \\
\text { all parameters. Report reasons or sources for distributions used to represent } \\
\text { uncertainty where appropriate. Providing a table to show the input values is } \\
\text { strongly recommended. }\end{array}$
\end{tabular}

Incremental costs and
outcomes

For each intervention, report mean values for the main categories of estimated costs and outcomes of interest, as well as mean differences between the comparator groups. If applicable, report incremental cost-effectiveness ratios.

\section{7}

Single study-based economic evaluation: Describe the effects of sampling un$776-777$

Characterising uncertainty certainty for the estimated incremental cost and incremental effectiveness parameters, together with the impact of methodological assumptions (such as discount rate, study perspective).

Model-based economic evaluation: Describe the effects on the results of uncertainty for all input parameters, and uncertainty related to the structure of the model and assumptions.

Characterising heterogeneity

If applicable, report differences in costs, outcomes, or cost-effectiveness that can be explained by variations between subgroups of patients with different baseline characteristics or other observed variability in effects that are not reducible by more information.

\section{Discussion}

Study findings, limitations, generalisability, and current knowledge
Summarise key study findings and describe how they support the conclusions reached. Discuss limitations and the generalisability of the findings and how the findings fit with current knowledge.

\section{Other}

Source of funding Describe how the study was funded and the role of the funder in the identification, design, conduct, and reporting of the analysis. Describe other non-monetary sources of support.

\section{Conflicts of interest} Describe any potential for conflict of interest of study contributors in accordance with journal policy. In the absence of a journal policy, we recommend authors comply with International Committee of Medical Journal Editors recommendations.

\section{Schechter 2008}

Title Identify the study as an economic evaluation or use more specific terms such
as "cost-effectiveness analysis", and describe the interventions compared.

Abstract

Provide a structured summary of objectives, perspective, setting, methods (inanalyses), and conclusions. 
Table 5. CHEERS checklist for methodological quality assessment of economic evaluations (Continued)

\author{
Introduction
}

\begin{tabular}{|c|c|c|}
\hline \multirow{2}{*}{$\begin{array}{l}\text { Background and ob- } \\
\text { jectives }\end{array}$} & Provide an explicit statement of the broader context for the study. & $763-764$ \\
\hline & $\begin{array}{l}\text { Present the study question and its relevance for health policy or practice deci- } \\
\text { sions. }\end{array}$ & 764 \\
\hline
\end{tabular}

\title{
Methods
}
Target population and Describe characteristics of the base case population and subgroups analysed, 764 subgroups including why they were chosen.

\begin{tabular}{lll}
\hline Setting and location & $\begin{array}{l}\text { State relevant aspects of the system(s) in which the decision(s) need(s) to be } \\
\text { made. }\end{array}$
\end{tabular}

\begin{tabular}{ll}
\hline Study perspective & $\begin{array}{l}\text { Describe the perspective of the study and relate this to the costs being evaluat- } \\
\text { ed. }\end{array}$
\end{tabular}

\begin{tabular}{lll}
\hline Comparators & $\begin{array}{l}\text { Describe the interventions or strategies being compared and state why they } \\
\text { were chosen. }\end{array}$ & 764
\end{tabular}

\begin{tabular}{lll}
\hline Time horizon & $\begin{array}{l}\text { State the time horizon(s) over which costs and consequences are being evalu- } \\
\text { ated and say why appropriate. }\end{array}$ & 764
\end{tabular}

\begin{tabular}{ll}
\hline Discount rate & $\begin{array}{l}\text { Report the choice of discount rate(s) used for costs and outcomes and say why } \\
\text { appropriate. }\end{array}$
\end{tabular}

\begin{tabular}{lll}
\hline $\begin{array}{l}\text { Choice of health out- } \\
\text { comes }\end{array}$ & $\begin{array}{l}\text { Describe what outcomes were used as the measure(s) of benefit in the evalua- } \\
\text { tion and their relevance for the type of analysis performed. }\end{array}$ & 764 \\
\hline
\end{tabular}

\begin{tabular}{lll}
\hline $\begin{array}{l}\text { Measurement of effec- } \\
\text { tiveness }\end{array}$ & $\begin{array}{l}\text { Single study-based estimates: Describe fully the design features of the single } \\
\text { effectiveness study and why the single study was a sufficient source of clinical } \\
\text { effectiveness data. }\end{array}$ & 764
\end{tabular}

Synthesis-based estimates: Describe fully the methods used for identification Not applicable of included studies and synthesis of clinical effectiveness data.

\begin{tabular}{lll}
\hline $\begin{array}{l}\text { Measurement and val- } \\
\text { uation of preference } \\
\text { based outcomes }\end{array}$ & $\begin{array}{l}\text { If applicable, describe the population and methods used to elicit preferences } \\
\text { for outcomes. }\end{array}$ & 765 \\
\hline $\begin{array}{l}\text { Estimating resources } \\
\text { and costs }\end{array}$ & $\begin{array}{l}\text { Single study-based economic evaluation: Describe approaches used to esti- } \\
\text { mate resource use associated with the alternative interventions. Describe pri- } \\
\text { mary or secondary research methods for valuing each resource item in terms } \\
\text { of its unit cost. Describe any adjustments made to approximate to opportunity } \\
\text { costs. }\end{array}$ & 764 \\
\hline $\begin{array}{l}\text { Currency, price date, } \\
\text { and conversion }\end{array}$ & $\begin{array}{l}\text { Report the dates of the estimated resource quantities and unit costs. Describe } \\
\text { methods for adjusting estimated unit costs to the year of reported costs if nec- } \\
\text { essary. Describe methods for converting costs into a common currency base } \\
\text { and the exchange rate. }\end{array}$ & 764 \\
\hline Choice of model & $\begin{array}{l}\text { Describe and give reasons for the specific type of decision-analytical model } \\
\text { used. Providing a figure to show model structure is strongly recommended. }\end{array}$ & Not applicable \\
\hline Assumptions & $\begin{array}{l}\text { Describe all structural or other assumptions underpinning the decision-analyt- } \\
\text { ical model. }\end{array}$ & Not applicable \\
\hline
\end{tabular}


Table 5. CHEERS checklist for methodological quality assessment of economic evaluations (Continued)

$\begin{array}{ll}\text { Analytical methods } & \text { Describe all analytical methods supporting the evaluation. This could include } \\ \text { methods for dealing with skewed, missing, or censored data; extrapolation } \\ \text { methods; methods for pooling data; approaches to validate or make adjust- } \\ \text { ments (such as half cycle corrections) to a model; and methods for handling } \\ \text { population heterogeneity and uncertainty. }\end{array}$

\section{Results}

$\begin{array}{ll}\text { Study parameters } & \text { Report the values, ranges, references, and, if used, probability distributions for } \\ \text { all parameters. Report reasons or sources for distributions used to represent } \\ \text { uncertainty where appropriate. Providing a table to show the input values is } \\ \text { strongly recommended. }\end{array}$

\begin{tabular}{ll}
\hline $\begin{array}{l}\text { Incremental costs and } \\
\text { outcomes }\end{array}$ & $\begin{array}{l}\text { For each intervention, report mean values for the main categories of estimated } \\
\text { costs and outcomes of interest, as well as mean differences between the com- } \\
\text { parator groups. If applicable, report incremental cost-effectiveness ratios. }\end{array}$
\end{tabular}
parator groups. If applicable, report incremental cost-effectiveness ratios.

$\begin{array}{ll}\text { Characterising uncer- } & \text { Single study-based economic evaluation: Describe the effects of sampling un- } \\ \text { tainty } & \text { certainty for the estimated incremental cost and incremental effectiveness pa- } \\ & \text { rameters, together with the impact of methodological assumptions (such as } \\ \text { discount rate, study perspective) }\end{array}$
discount rate, study perspective)

Model-based economic evaluation: Describe the effects on the results of uncertainty for all input parameters, and uncertainty related to the structure of the model and assumptions.

$\begin{array}{ll}\text { Characterising hetero- } & \text { If applicable, report differences in costs, outcomes, or cost-effectiveness that } \\ \text { geneity } & \begin{array}{l}\text { can be explained by variations between subgroups of patients with different } \\ \text { baseline characteristics or other observed variability in effects that are not re- } \\ \text { ducible by more information. }\end{array}\end{array}$
are not reducible by more information.

\section{Discussion}

Study findings, limita- Summarise key study findings and describe how they support the conclusions

\section{Other}

Source of funding Describe how the study was funded and the role of the funder in the identification, design, conduct, and reporting of the analysis. Describe other non-monetary sources of support.

\section{Conflicts of interest} dance with journal policy. In the absence of a journal policy, we recommend authors comply with International Committee of Medical Journal Editors recommendations.

\section{Wagner 2001}

Title Identify the study as an economic evaluation or use more specific terms such Not reported
as "cost-effectiveness analysis", and describe the interventions compared.


Table 5. CHEERS checklist for methodological quality assessment of economic evaluations (Continued)
Abstract
Provide a structured summary of objectives, perspective, setting, methods (in- cluding study design and inputs), results (including base case and uncertainty
Not reported analyses), and conclusions.

\begin{tabular}{l} 
Introduction \\
\hline $\begin{array}{l}\text { Background and ob- } \\
\text { jectives }\end{array}$
\end{tabular}

Provide an explicit statement of the broader context for the study.

Present the study question and its relevance for health policy or practice deci-

695 sions.

\begin{tabular}{|c|c|c|}
\hline \multicolumn{3}{|l|}{ Methods } \\
\hline $\begin{array}{l}\text { Target population and } \\
\text { subgroups }\end{array}$ & $\begin{array}{l}\text { Describe characteristics of the base case population and subgroups analysed, } \\
\text { including why they were chosen. }\end{array}$ & 697 \\
\hline Setting and location & $\begin{array}{l}\text { State relevant aspects of the system(s) in which the decision(s) need(s) to be } \\
\text { made. }\end{array}$ & $695-696$ \\
\hline Study perspective & $\begin{array}{l}\text { Describe the perspective of the study and relate this to the costs being evaluat- } \\
\text { ed. }\end{array}$ & Not reported \\
\hline Comparators & $\begin{array}{l}\text { Describe the interventions or strategies being compared and state why they } \\
\text { were chosen. }\end{array}$ & Not reported \\
\hline Time horizon & $\begin{array}{l}\text { State the time horizon(s) over which costs and consequences are being evalu- } \\
\text { ated and say why appropriate. }\end{array}$ & Not reported \\
\hline Discount rate & $\begin{array}{l}\text { Report the choice of discount rate(s) used for costs and outcomes and say why } \\
\text { appropriate. }\end{array}$ & Not reported \\
\hline $\begin{array}{l}\text { Choice of health out- } \\
\text { comes }\end{array}$ & $\begin{array}{l}\text { Describe what outcomes were used as the measure(s) of benefit in the evalua- } \\
\text { tion and their relevance for the type of analysis performed. }\end{array}$ & Not reported \\
\hline \multirow[t]{2}{*}{$\begin{array}{l}\text { Measurement of effec- } \\
\text { tiveness }\end{array}$} & $\begin{array}{l}\text { Single study-based estimates: Describe fully the design features of the single } \\
\text { effectiveness study and why the single study was a sufficient source of clinical } \\
\text { effectiveness data. }\end{array}$ & Not reported \\
\hline & $\begin{array}{l}\text { Synthesis-based estimates: Describe fully the methods used for identification } \\
\text { of included studies and synthesis of clinical effectiveness data. }\end{array}$ & Not applicable \\
\hline $\begin{array}{l}\text { Measurement and val- } \\
\text { uation of preference } \\
\text { based outcomes }\end{array}$ & $\begin{array}{l}\text { If applicable, describe the population and methods used to elicit preferences } \\
\text { for outcomes. }\end{array}$ & Not applicable \\
\hline $\begin{array}{l}\text { Estimating resources } \\
\text { and costs }\end{array}$ & $\begin{array}{l}\text { Single study-based economic evaluation: Describe approaches used to esti- } \\
\text { mate resource use associated with the alternative interventions. Describe pri- } \\
\text { mary or secondary research methods for valuing each resource item in terms } \\
\text { of its unit cost. Describe any adjustments made to approximate to opportunity } \\
\text { costs. }\end{array}$ & Not reported \\
\hline $\begin{array}{l}\text { Currency, price date, } \\
\text { and conversion }\end{array}$ & $\begin{array}{l}\text { Report the dates of the estimated resource quantities and unit costs. Describe } \\
\text { methods for adjusting estimated unit costs to the year of reported costs if nec- } \\
\text { essary. Describe methods for converting costs into a common currency base } \\
\text { and the exchange rate. }\end{array}$ & Not reported \\
\hline
\end{tabular}


Table 5. CHEERS checklist for methodological quality assessment of economic evaluations (Continued)

Choice of model Describe and give reasons for the specific type of decision-analytical model used. Providing a figure to show model structure is strongly recommended.

Not applicable

\begin{tabular}{ll}
\hline Assumptions & $\begin{array}{l}\text { Describe all structural or other assumptions underpinning the decision-analyt- } \\
\text { ical model. }\end{array}$
\end{tabular}
ical model.

$\begin{array}{ll}\text { Analytical methods } & \text { Describe all analytical methods supporting the evaluation. This could include } \\ \text { methods for dealing with skewed, missing, or censored data; extrapolation } \\ \text { methods; methods for pooling data; approaches to validate or make adjust- } \\ \text { ments (such as half cycle corrections) to a model; and methods for handling } \\ \text { population heterogeneity and uncertainty. }\end{array}$

Not reported population heterogeneity and uncertainty.

\section{Results}

Study parameters

Report the values, ranges, references, and, if used, probability distributions for

$697-698$ all parameters. Report reasons or sources for distributions used to represent uncertainty where appropriate. Providing a table to show the input values is strongly recommended.

Incremental costs and
outcomes

For each intervention, report mean values for the main categories of estimated costs and outcomes of interest, as well as mean differences between the comparator groups. If applicable, report incremental cost-effectiveness ratios.
Not reported

Single study-based economic evaluation: Describe the effects of sampling uncertainty for the estimated incremental cost and incremental effectiveness parameters, together with the impact of methodological assumptions (such as discount rate, study perspective).

Not reported

\section{tainty}

Model-based economic evaluation: Describe the effects on the results of uncertainty for all input parameters, and uncertainty related to the structure of the model and assumptions
If applicable, report differences in costs, outcomes, or cost-effectiveness that can be explained by variations between subgroups of patients with different baseline characteristics or other observed variability in effects that are not reducible by more information.
Characterising heterogeneity

\section{Not reported}

Not reported

\section{Discussion}

Summarise key study findings and describe how they support the conclusions reached. Discuss limitations and the generalisability of the findings and how
$698-699$

\section{ty, and current knowl- the findings fit with current knowledge.}

edge

Other

Source of funding Describe how the study was funded and the role of the funder in the identification, design, conduct, and reporting of the analysis. Describe other non-monetary sources of support.

\section{Conflicts of interest}

Describe any potential for conflict of interest of study contributors in accordance with journal policy. In the absence of a journal policy, we recommend Not reported authors comply with International Committee of Medical Journal Editors recommendations. 
Table 6. Results of subgroup analysis

\begin{tabular}{llll}
\hline Subgroup category & $\mathrm{N}$ & RD $(95 \% \mathrm{Cl})$ & $12 \%$ \\
& studies & & \\
\hline
\end{tabular}

\section{QI Strategy}

\begin{tabular}{lccc}
\hline Audit and feedback & 11 & 0.12 (0.06 to 0.18) & 89 \\
\hline Case management & 18 & $0.14(0.07$ to 0.21$)$ & 94 \\
\hline Team changes & 19 & $0.20(0.13$ to 0.26$)$ & 88 \\
\hline Electronic patient registry & 10 & $0.18(0.07$ to 0.29$)$ & 94 \\
\hline Clinician education & 16 & $0.13(0.07$ to 0.19$)$ & 95 \\
\hline Clinician reminders & 10 & $0.13(0.05$ to 0.21$)$ & 85 \\
\hline Patient Education & 30 & $0.15(0.13$ to 0.18$)$ & 95 \\
\hline Promotion of self-management & 21 & $0.19(0.13$ to 0.26$)$ & 96 \\
\hline Patient reminders & 16 & $0.11(0.07$ to 0.14$)$ & 93 \\
\hline BCT (patients) & &
\end{tabular}

\begin{tabular}{|c|c|c|c|}
\hline Goal setting (Outcome) & 14 & $0.26(0.16$ to 0.36$)$ & 93 \\
\hline $\begin{array}{l}\text { Feedback on outcomes of behav- } \\
\text { iour/biofeedback }\end{array}$ & 15 & 0.19 (0.13 to 0.25$)$ & 80 \\
\hline Credible source & 10 & 0.22 (0.06 to 0.38$)$ & 95 \\
\hline Prompts/cues & 25 & $0.11(0.07$ to 0.14$)$ & 92 \\
\hline Social support (unspecified) & 14 & 0.19 (0.09 to 0.28$)$ & 93 \\
\hline Problem solving & 10 & 0.17 (0.08 to 0.27$)$ & 89 \\
\hline Restructuring the social environment & 17 & 0.17 (0.10 to 0.24$)$ & 85 \\
\hline Instruction on how to perform behaviour & 34 & $0.13(0.11$ to 0.15$)$ & 94 \\
\hline Social support (practical) & 20 & 0.14 (0.09 to 0.20$)$ & 90 \\
\hline Information about health consequences & 19 & $0.12(0.07$ to 0.16$)$ & 92 \\
\hline \multicolumn{4}{|l|}{ BCT (healthcare professionals) } \\
\hline Restructuring the social environment & 23 & $0.19(0.12$ to 0.26$)$ & 91 \\
\hline Credible source & 13 & $0.16(0.08$ to 0.24$)$ & 95 \\
\hline Adding objects to the environment & 15 & $0.14(0.07$ to 0.20$)$ & 88 \\
\hline Social support (practical) & 10 & $0.13(0.03$ to 0.22$)$ & 87 \\
\hline
\end{tabular}


Table 6. Results of subgroup analysis (Continued)

\begin{tabular}{llll} 
Instruction on how to perform behaviour & 30 & 0.13 (0.08 to 0.17) & 93 \\
\hline Prompts/cues & 15 & 0.12 (0.06 to 0.17$)$ & 85 \\
\hline $\begin{array}{l}\text { Feedback on outcomes of behav- } \\
\text { iour/biofeedback }\end{array}$ & 17 & $0.11(0.07$ to 0.16$)$ & 81 \\
\hline
\end{tabular}

\section{APPENDICES}

\section{Appendix 1. CENTRAL and NHS EED search strategy}

\#1 MeSH descriptor: [Diabetes Mellitus] explode all trees \#2 MeSH descriptor: [Diabetes Complications] explode all trees \#3 MeSH descriptor: [Diabetic Retinopathy] explode all trees \#4 (diabet* or proliferative or non-proliferative) near/4 retinopath* \#5 diabet $^{\star}$ near $/ 3$ (eye ${ }^{\star}$ or vision or visual ${ }^{\star}$ or sight ${ }^{\star}$ ) \#6 retinopath* near/3 (eye ${ }^{\star}$ or vision or visual ${ }^{\star}$ or sight ${ }^{\star}$ ) \#7 DR near/3 (eye* or vision or visual ${ }^{\star}$ or sight ${ }^{\star}$ ) $\# 8 \# 1$ or \#2 or \#3 or \#4 or \#5 or \#6 or \#7 \#9 MeSH descriptor: [Mass Screening] explode all trees \#10 MeSH descriptor: [Vision Tests] explode all trees \#11 MeSH descriptor: [Telemedicine] explode all trees \#12 MeSH descriptor: [Photography] explode all trees \#13 MeSH descriptor: [Ophthalmoscopes] explode all trees \#14 MeSH descriptor: [Ophthalmoscopy] explode all trees

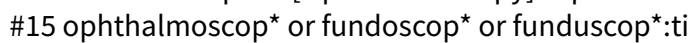
\#16 (exam* or photo* or imag ${ }^{\star}$ ) near/3 fundus \#17 photography or retinography \#18 (mydriatic or digital or retina* or fundus or steroscopic) near/3 camera* \#19 (mydriatic or digital or retina* or fundus or steroscopic) near/3 imag* \#20 screen\$.tw.

\#21 (eye* or retina* or ophthalm*) near/4 exam* \#22 (eye* or vision or retinopathy or ophthalmic) near/4 test*

\#23 (eye* or retina* or ophthalm*) near/4 visit*

\#24 MeSH descriptor: [Office Visits] this term only \#25 (telemedicine* or telemonitor ${ }^{\star}$ or telescreen* or telehealth or teleophthalmology)

$\# 26 \# 9$ or \#10 or \#11 or \#12 or \#13 or \#14 or \#15 or \#16 or \#17 or \#18 or \#19 or \#20 or \#21 or \#22 or \#23 or \#24 or \#25 \#27 MeSH descriptor: [Quality of Health Care] explode all trees \#28 MeSH descriptor: [Quality of Health Care] this term only \#29 MeSH descriptor: [Quality Improvement] this term only \#30 MeSH descriptor: [Delivery of Health Care] this term only \#31 MeSH descriptor: [Delivery of Health Care, Integrated] this term only \#32 service delivery \#33 decision making \#34 consensus near/3 (process* or discuss) \#35 stakeholder* \#36 MeSH descriptor: [Quality Control] this term only \#37 MeSH descriptor: [Total Quality Management] this term only \#38 MeSH descriptor: [Quality Indicators, Health Care] this term only \#39 MeSH descriptor: [Quality Assurance, Health Care] this term only \#40 quality assurance \#41 quality near/2 improv* \#42 total quality \#43 continuous quality \#44 quality management \#45 (organisation* near/3 cultur ${ }^{\star}$ ) \#46 MeSH descriptor: [Disease Management] this term only 
\#47 MeSH descriptor: [Program Evaluation] this term only

\#48 (provider ${ }^{\star}$ or program ${ }^{\star}$ ) near/3 (monitor ${ }^{\star}$ or evaluate* or modif* $^{\star}$ or practice)

\#49 implement ${ }^{\star}$ near/3 (improve* or change* or effort* or issue* or impede* or glossary or tool ${ }^{\star}$ or innovation* or outcome* or driv* or examin $^{\star}$ or reexamin ${ }^{\star}$ or scale or strateg $^{\star}$ or advis ${ }^{\star}$ or expert $^{\star}$ )

\#50 needs near/3 assess*

\#51 (education ${ }^{\star}$ or learn ${ }^{\star}$ ) near/5 (continu* or material* ${ }^{\star}$ or meeting or collaborat ${ }^{\star}$ )

\#52 MeSH descriptor: [Medical Audit] explode all trees

\#53 audit or feedback or compliance or adherence or training or innovation:ti

\#54 guideline* near/3 (clinical or practice or implement* or promot ${ }^{\star}$ )

\#55 MeSH descriptor: [Health Services Accessibility] explode all trees

\#56 outreach near/2 (service\$ or visit*)

\#57 intervention ${ }^{\star}$ near/3 (no or usual or routine or target ${ }^{\star}$ or tailor $^{\star}$ or mediat $^{\star}$ )

\#58 usual care

$\# 59 \# 27$ or \#28 or \#29 or \#30 or \#31 or \#32 or \#33 or \#34 or \#35 or \#36 or \#37 or \#38 or \#39 or \#40 or \#41 or \#42 or \#43 or \#44 or \#45 or \#46 or \#47 or \#48 or \#49 or \#50 or \#51 or \#52 or \#53 or \#54 or \#55 or \#56 or \#57 or \#58

\#60 MeSH descriptor: [Reminder Systems] explode all trees

\#61 remind*

\#62 improve* near/3 (attend* or visit* or intervention $^{\star}$ or adhere ${ }^{\star}$ )

\#63 increas ${ }^{\star}$ near/3 (attend ${ }^{\star}$ or visit* or intervention* or adhere ${ }^{\star}$ )

\#64 appointment ${ }^{\star}$ near/3 (miss* or fail ${ }^{\star}$ or remind ${ }^{\star}$ or follow up)

\#65 MeSH descriptor: [Telephone] this term only

\#66 telephone*

\#67 MeSH descriptor: [Cell Phones] this term only

\#68 MeSH descriptor: [Mobile Applications] this term only

\#69 MeSH descriptor: [Remote Consultation] this term only

\#70 m-health or e-health or g-health or u-health

\#71 phone* near/1 (smart or cell)

\#72 smartphone* or cellphone ${ }^{\star}$

\#73 hand held device*

\#74 mobile near/2 (health or healthcare or phone ${ }^{\star}$ or device ${ }^{\star}$ or monitor ${ }^{\star}$ or comput ${ }^{\star}$ or app or apps or application)

\#75 MeSH descriptor: [Internet] this term only

\#76 MeSH descriptor: [Social Networking] this term only

\#77 email $^{\star}$ or text* or message*

$\# 78$ letter or mail or mailed or print* or brochure* or newsletter*

$\# 79 \# 60$ or \#61 or \#62 or \#63 or \#64 or \#65 or \#66 or \#67 or \#68 or \#69 or \#70 or \#71 or \#72 or \#73 or \#74 or \#75 or \#76 or \#77 or \#78

\#80 MeSH descriptor: [Primary Health Care] this term only

\#81 MeSH descriptor: [General Practitioners] this term only

\#82 MeSH descriptor: [Physicians, Family] this term only

\#83 MeSH descriptor: [Physicians, Primary Care] this term only

\#84 MeSH descriptor: [Primary Prevention] this term only

\#85 MeSH descriptor: [Preventive Health Services] this term only

\#86 MeSH descriptor: [Community Health Services] this term only

\#87 MeSH descriptor: [Nurses, Community Health] this term only

\#88 MeSH descriptor: [Health Services, Indigenous] this term only

\#89 MeSH descriptor: [Rural Health Services] explode all trees

\#90 MeSH descriptor: [Mobile Health Units] this term only

\#91 Ophthalmologist* or Optometrist ${ }^{\star}$ or Optician* or Orthopist ${ }^{\star}$ or Refractionists

\#92 (Ophthalmic or eye) near/3 (surgeon ${ }^{\star}$ or nurse* or technician* or officer* or assistant ${ }^{\star}$ or staff*)

\#93 MeSH descriptor: [Physician's Practice Patterns] this term only

\#94 MeSH descriptor: [Professional Practice] this term only

\#95 MeSH descriptor: [Education, Medical, Continuing] this term only

\#96 MeSH descriptor: [Nurses] explode all trees

\#97 MeSH descriptor: [Specialties, Nursing] this term only

\#98 MeSH descriptor: [Nurse's Role] this term only

\#99 MeSH descriptor: [Education, Nursing, Continuing] this term only

$\# 100$ nurse or nurses

\#101 MeSH descriptor: [Pharmacists] this term only

\#102 pharmacist*

\#103 (role or roles) near/3 expan*

$\# 104$ task $^{\star}$ near/3 shift ${ }^{*}$

\#105 MeSH descriptor: [Medical Records Systems, Computerized] explode all trees

\#106 MeSH descriptor: [Management Information Systems] this term only 
\#107 MeSH descriptor: [Database Management Systems] this term only

\#108 MeSH descriptor: [Computer Systems] this term only

\#109 MeSH descriptor: [Point-of-Care Systems] this term only

\#110 MeSH descriptor: [Hospital Information Systems] this term only

$\# 111$ (health or healthcare) near/4 (record or management system ${ }^{\star}$ )

\#112 (decision near/5 support) .ti.

$\# 113 \# 80$ or \#81 or \#82 or \#83 or \#84 or \#85 or \#86 or \#87 or \#88 or \#89 or \#90 or \#91 or \#92 or \#93 or \#94 or \#95 or \#96 or \#97 or \#98 or \#99

or \#100 or \#101 or \#102 or \#103 or \#104 or \#105 or \#106 or \#107 or \#108 or \#109 or \#110 or \#111 or \#112

\#114 MeSH descriptor: [Economics] this term only

\#115 MeSH descriptor: [Costs and Cost Analysis] this term only

\#116 MeSH descriptor: [Cost Allocation] this term only

\#117 MeSH descriptor: [Cost-Benefit Analysis] this term only

\#118 MeSH descriptor: [Cost Control] this term only

\#119 MeSH descriptor: [Cost Savings] this term only

\#120 MeSH descriptor: [Cost of Illness] explode all trees

\#121 MeSH descriptor: [Cost Sharing] this term only

\#122 MeSH descriptor: [Deductibles and Coinsurance] this term only

\#123 MeSH descriptor: [Medical Savings Accounts] this term only

\#124 MeSH descriptor: [Health Care Costs] this term only

\#125 MeSH descriptor: [Direct Service Costs] this term only

\#126 MeSH descriptor: [Drug Costs] this term only

\#127 MeSH descriptor: [Employer Health Costs] this term only

\#128 MeSH descriptor: [Hospital Costs] this term only

\#129 MeSH descriptor: [Health Expenditures] this term only

\#130 MeSH descriptor: [Capital Expenditures] this term only

\#131 MeSH descriptor: [Economics, Hospital] explode all trees

\#132 MeSH descriptor: [Economics, Medical] explode all trees

\#133 MeSH descriptor: [Economics, Nursing] this term only

\#134 MeSH descriptor: [Economics, Pharmaceutical] this term only

\#135 MeSH descriptor: [Fees and Charges] explode all trees

\#136 MeSH descriptor: [Budgets] explode all trees

\#137 low* near/2 cost*

\#138 high* near $/ 2$ cost $^{*}$

\#139 (health care or healthcare) near/2 cost*

\#140 fiscal or funding or financial or finance

\#141 cost near/2 estimate*

\#142 cost near/2 variable*

\#143 unit near/2 cost $^{\star}$

$\# 144$ economic $^{*}$ or pharmacoeconomic* or price* or pricing

\#145 MeSH descriptor: [Uncompensated Care] this term only

\#146 MeSH descriptor: [Reimbursement Mechanisms] this term only

\#147 MeSH descriptor: [Reimbursement, Incentive] this term only

$\# 148$ insurance near/3 (health or scheme ${ }^{\star}$ )

\#149 financial or economic or pay or payment or copayment or paid or fee or fees or monetary or money or cash or incentiv ${ }^{\star}$ or disincentiv* $\# 150 \# 114$ or \#115 or \#116 or \#117 or \#118 or \#119 or \#120 or \#121 or \#122 or \#123 or \#124 or \#125 or \#126 or \#127 or \#128 or \#129 or $\# 130$ or \#131 or \#132 or \#133 or \#134 or \#135 or \#136 or \#137 or \#138 or \#139 or \#140 or \#141 or \#142 or \#143 or \#144 or \#145 or \#146 or

$\# 147$ or \#148 or \#149

\#151 \#59 or \#79 or \#113 or \#150

\#152 MeSH descriptor: [Patient Acceptance of Health Care] explode all trees

\#153 MeSH descriptor: [Attitude to Health] explode all trees

\#154 MeSH descriptor: [Health Behavior] explode all trees

\#155 barrier* $^{\star}$ or obstacle* or facilitat* or enable*

\#156 uptake or takeup or attend ${ }^{\star}$ or accept ${ }^{\star}$ or adhere ${ }^{\star}$ or attitude ${ }^{\star}$ or participat ${ }^{\star}$ or facilitat ${ }^{\star}$ or utilisat ${ }^{\star}$ or utilizat $^{*}$

$\# 157$ complie $^{\star}$ or comply or compliance* or noncompliance* or non compliance*

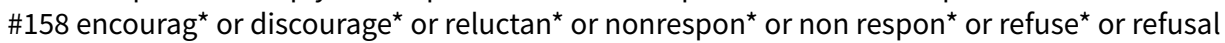

\#159 non-attend* or non attend* or dropout or drop out or apath*

\#160 MeSH descriptor: [Health Education] this term only

\#161 MeSH descriptor: [Patient Education as Topic] explode all trees

\#162 MeSH descriptor: [Health Promotion] explode all trees

\#163 health near/2 (promotion* or knowledge or belief*)

\#164 educat ${ }^{\star}$ near/2 (intervention* or information or material or leaflet)

\#165 MeSH descriptor: [Socioeconomic Factors] this term only 
\#166 MeSH descriptor: [Poverty] explode all trees

\#167 MeSH descriptor: [Social Class] this term only

\#168 MeSH descriptor: [Educational Status] this term only

\#169 (school or education ${ }^{\star}$ ) near/3 (status or level ${ }^{\star}$ or attain* or achieve*)

\#170 MeSH descriptor: [Employment] this term only

\#171 MeSH descriptor: [Healthcare Disparities] this term only

\#172 MeSH descriptor: [Health Status Disparities] this term only

\#173 MeSH descriptor: [Medically Underserved Area] explode all trees

\#174 MeSH descriptor: [Rural Population] this term only

\#175 MeSH descriptor: [Urban Population] this term only

\#176 MeSH descriptor: [Ethnic Groups] explode all trees

\#177 MeSH descriptor: [Minority Groups] this term only

\#178 MeSH descriptor: [Vulnerable Populations] this term only

$\# 179$ (health* or social $^{\star}$ or racial ${ }^{\star}$ or ethnic ${ }^{\star}$ ) near/5 (inequalit ${ }^{\star}$ or inequit* or disparit $^{\star}$ or equit* or disadvantage or depriv $^{\star}$ )

$\# 180$ disadvant $^{\star}$ or marginali* or underserved or under served or impoverish* or minorit $^{\star}$ or racial* or ethnic ${ }^{\star}$

$\# 181 \# 152$ or \#153 or \#154 or \#155 or \#156 or \#157 or \#158 or \#159 or \#160 or \#161 or \#162 or \#163 or \#164 or \#165 or \#166 or \#167 or \#168 or \#169 or \#170 or \#171 or \#172 or \#173 or \#174 or \#175 or \#176 or \#177 or \#178 or \#179 or \#180

\#182 \#151 or \#181

$\# 183$ \#8 and \#26 and \#182

\#184 (ranibizumab or bevacizumab or avastin or aflibercept or photocoagulation or coronary or cardiovascular):ti

\#185 blood glucose or blood pressure:ti

\#186 macula* near/2 (oedema or edema):ti

$\# 187$ \#184 or \#185 or \#186

\#188 \#183 not \#187

\section{Appendix 2. MEDLINE Ovid search strategy}

1. randomized controlled trial.pt.

2. random\$.ab,ti.

3. placebo.ab,ti.

4. dt.fs.

5. trial.ab,ti.

6. (group or groups).ab,ti.

7. or/1-6

8. exp animals/

9. exp humans/

10. 8 not (8 and 9)

11.7 not 10

12. exp Randomized Controlled Trials as Topic/

13. 11 or 12

14. exp Diabetes Mellitus/

15. exp Diabetes Complications/

16. exp Diabetic Retinopathy/

17. ((diabet\$ or proliferative or non-proliferative) adj4 retinopath\$).tw.

18. diabetic retinopathy.kw.

19. (diabet\$ adj3 (eye\$ or vision or visual\$ or sight\$)).tw.

20. (retinopath\$ adj3 (eye or vision or visual\$ or sight\$)).tw.

21. (DR adj3 (eye\$ or vision or visual\$ or sight\$)).tw.

22. or/14-21

23. exp Mass Screening/

24. exp Vision Tests/

25. exp Telemedicine/

26. exp Photography/

27. exp Ophthalmoscopes/

28. exp Ophthalmoscopy/

29. (ophthalmoscop\$ or fundoscop\$ or funduscop\$).ti.

30. ((exam\$ or photo $\$$ or imag\$) adj3 fundus).tw.

31. (photography or retinography).tw.

32. ((mydriatic or digital or retina\$ or fundus or steroscopic) adj3 camera).tw.

33. ((mydriatic or digital or retina\$ or fundus or steroscopic) adj3 imag\$).tw.

34. screen\$.tw.

35. ((eye\$ or retina\$ or ophthalm\$) adj4 exam\$).tw. 
36. ((eye or vision or retinopathy or ophthalmic) adj4 test\$).tw.

37. ((eye\$ or retina $\$$ or ophthalm\$) adj4 visit\$).tw.

38. Office Visits/

39. (telemedicine\$ or telemonitor\$ or telescreen\$ or telehealth or teleophthalmology).tw.

40. or/23-39

41. "Quality of Health Care"/

42. Quality Improvement/

43. Delivery of Health Care/

44. Delivery of Health Care, Integrated/

45. service delivery.tw.

46. decision making.tw.

47. (consensus adj3 (process $\$$ or discuss)).tw.

48. stakeholder\$.tw.

49. Quality Control/

50. Total Quality Management/

51. Quality Indicators, Health Care/

52. Quality Assurance, Health Care/

53. quality assurance.tw.

54. (quality adj2 improv\$).tw.

55. total quality.tw.

56. continuous quality.tw.

57. quality management.tw.

58. (organisation $\$$ adj3 cultur\$).tw.

59. Disease Management/

60. Program Evaluation/

61. ((provider\$ or program\$) adj3 (monitor\$ or evaluate\$ or modif\$ or practice)).tw.

62. (implement $\$$ adj3 (improve $\$$ or change $\$$ or effort $\$$ or issue $\$$ or impede $\$$ or glossary or tool $\$$ or innovation $\$$ or outcome $\$$ or driv $\$$ or examin $\$$ or reexamin $\$$ or scale $\$$ or strateg\$ or advis\$ or expert\$)).tw.

63. (need\$ adj3 assess\$).tw.

64. ((education\$ or learn\$) adj5 (continu\$ or material\$ or meeting or collaborat\$)).tw.

65. exp Medical audit/

66. (audit or feedback or compliance or adherence or training or innovation).ti.

67. (guideline adj3 (clinical or practice or implement\$ or promot\$)).tw.

68. exp Health Services Accessibility/

69. (outreach adj2 (service or visit\$)).tw.

70. (intervention $\$$ adj3 (no or usual or routine or target\$ or tailor $\$$ or mediat\$)).tw.

71. usual care.tw.

72. exp Reminder Systems/

73. remind\$.tw.

74. (improve\$ adj3 (attend\$ or visit\$ or intervention\$ or adhere\$)).tw.

75. (increas\$ adj3 (attend\$ or visit\$ or intervention $\$$ or adhere\$)).tw.

76. (appointment $\$$ adj3 (miss\$ or fail\$ or remind\$ or follow up)).tw.

77. Telephone/

78. telephone.tw.

79. Cell Phones/

80. Mobile Applications/

81. Remote Consultation/

82. (m-health or e-health or g-health or u-health).tw.

83. (phone\$ adj1 (smart or cell)).tw.

84. (smartphone\$ or cellphone\$).tw.

85. (hand adj1 held device\$).tw.

86. (mobile adj2 (health or healthcare or phone\$ or device $\$$ or monitor $\$$ or comput $\$$ or app or apps or application)).tw.

87. Internet/

88. Social Networking/

89. (email\$ or text\$ or message\$).tw.

90. (letter or mail or mailed or print $\$$ or brochure\$ or newsletter\$).tw.

91. Primary Health Care/

92. General Practitioners/ or Physicians, Family/ or Physicians, Primary Care/

93. Primary Prevention/

94. Preventive Health Services/

95. Community Health Services/

96. Community Health Nursing/

Interventions to increase attendance for diabetic retinopathy screening (Review)

Copyright @ 2018 The Cochrane Collaboration. Published by John Wiley \& Sons, Ltd. 
97. Health Services, Indigenous/

98. Rural Health Services/

99. Mobile Health Units/

100. (Ophthalmologist\$ or Optometrist\$ or Optician\$ or Orthopist\$ or Refractionists).tw.

101. ((Ophthalmic or eye) adj3 (surgeon $\$$ or nurse $\$$ or technician $\$$ or officer $\$$ or assistant $\$$ or staff\$)).tw.

102. Physician's Practice Patterns/

103. Professional Practice/

104. (professional adj3 (practice or develop\$ or educat)).tw.

105. Education, Medical, Continuing/

106. exp nurses/

107. Specialties, Nursing/

108. Nurse's Role/

109. Education, Nursing, Continuing/

110. (nurse or nurses).tw.

111. Pharmacists/

112. pharmacist\$.tw.

113. ((role or roles) adj3 expan\$).tw.

114. (task\$ adj3 shift\$).tw.

115. exp Medical Records Systems, Computerized/

116. Management Information Systems/

117. Database Management Systems/

118. Computer Systems/

119. Point-of-Care Systems/

120. Hospital Information Systems/

121. ((health or healthcare) adj4 (record or management system\$)).tw.

122. (decision adj5 support).ti.

123. Economics/

124. "costs and cost analysis"/

125. Cost allocation/

126. Cost-benefit analysis/

127. Cost control/

128. Cost savings/

129. Cost of illness/

130. Cost sharing/

131. "deductibles and coinsurance"/

132. Medical savings accounts/

133. Health care costs/

134. Direct service costs/

135. Drug costs/

136. Employer health costs/

137. Hospital costs/

138. Health expenditures/

139. Capital expenditures/

140. Value of life/

141. exp economics, hospital/

142. exp economics, medical/

143. Economics, nursing/

144. Economics, pharmaceutical/

145. exp "fees and charges"/

146. exp budgets/

147. (low adj cost).mp.

148. (high adj cost).mp.

149. (health?care adj cost\$).mp.

150. (fiscal or funding or financial or finance).tw.

151. (cost adj estimate\$).mp.

152. (cost adj variable).mp.

153. (unit adj cost\$).mp.

154. (economic $\$$ or pharmacoeconomic $\$$ or price $\$$ or pricing).tw.

155. Uncompensated Care/

156. Reimbursement Mechanisms/

157. Reimbursement, Incentive/

158. (insurance adj3 (health\$ or scheme\$)).tw.

Interventions to increase attendance for diabetic retinopathy screening (Review)

Copyright $\odot 2018$ The Cochrane Collaboration. Published by John Wiley \& Sons, Ltd. 
159. (financial or economic or pay or payment or copayment or paid or fee or fees or monetary or money or cash or incentiv\$ or disincentiv \$).tw.

160. or/41-159

161. exp Patient Acceptance of health Care/

162. exp Attitude to Health/

163. exp Health Behavior/

164. (barrier\$ or obstacle\$ or facilitat\$ or enable\$).tw.

165. (uptake or takeup or attend\$ or accept\$ or adhere\$ or attitude $\$$ or participat $\$$ or facilitat $\$$ or utilisat $\$$ or utilizat\$).tw.

166. (complie or comply or compliance $\$$ or noncompliance $\$$ or non compliance\$).tw.

167. (encourag $\$$ or discourage $\$$ or reluctan $\$$ or nonrespon $\$$ or non respon $\$$ or refuse $\$$ ).tw.

168. (non-attend\$ or non attend\$ or dropout or drop out or apath\$).tw.

169. Health Education/

170. exp Patient Education as Topic/

171. exp Health Promotion/

172. exp Counseling/

173. "Attitude of Health Personnel"/

174. (health adj2 (promotion\$ or knowledge or belief\$)).tw.

175. (educat\$ adj2 (intervention\$ or information or material or leaflet)).tw.

176. Socioeconomic Factors/

177. exp Poverty/

178. Social Class/

179. Educational Status/

180. ((school or education\$) adj3 (status or level\$ or attain\$ or achieve\$)).tw.

181. Employment/

182. Healthcare Disparities/

183. Health Status Disparities/

184. exp Medically Underserved Area/

185. Rural Population/

186. Urban Population/

187. exp Ethnic Groups/

188. Minority Groups/

189. Vulnerable Populations/

190. ((health\$ or social\$ or racial\$ or ethnic\$) adj5 (inequalit\$ or inequit\$ or disparit\$ or equit $\$$ or disadvantage $\$$ or depriv\$)).tw.

191. (disadvant or marginali\$ or underserved or under served or impoverish\$ or minorit\$ or racial\$ or ethnic\$).tw.

192. or/161-191

193. 160 or 192

194. 13 and 22 and 40 and 193

195. (ranibizumab or bevacizumab or avastin or aflibercept or photocoagulation or coronary or cardiovascular).ti.

196. (blood glucose or blood pressure).ti.

197. (macula\$ adj2 (oedema or edema)).ti.

198. (cataract or intraocular or glaucoma).ti.

199. macula\$ degeneration.ti.

200. nerve fiber layer.ti.

201. or/195-200

202. 194 not 201

The search filter for trials at the beginning of the MEDLINE strategy is from the published paper by Glanville 2006.

\section{Appendix 3. Embase Ovid search strategy}

1. exp randomized controlled trial/

2. exp randomization/

3. exp double blind procedure/

4. exp single blind procedure/

5. or/1-4

6. (animal or animal experiment).sh.

7. human.sh.

8. 6 and 7

9.6 not 8

10.5 not 9

11. exp clinical trial/

12. (clin\$ adj3 trial\$).tw. 
13. random\$.tw.

14. exp placebo/

15. placebo\$.tw.

16. ((singl\$ or doubl\$ or trebl\$ or tripl\$) adj3 (blind\$ or mask\$)).tw.

17. exp experimental design/

18. exp crossover procedure/

19. exp control group/

20. exp latin square design/

21. or $/ 11-20$

22. 21 not 9

23. 22 not 10

24. exp comparative study/

25. exp evaluation/

26. exp prospective study/

27. (control\$ or prospectiv\$ or volunteer\$).tw.

28. or/24-27

29. 28 not 9

30. 29 not ( 10 or 22 )

31.10 or 23 or 30

32. "randomized controlled trial (topic)"/

33. 31 or 32

34. exp diabetes mellitus/

35. exp diabetic retinopathy/

36. ((diabet\$ or proliferative or non-proliferative) adj4 retinopath\$).tw.

37. diabetic retinopathy.kw.

38. (diabet\$ adj3 (eye\$ or vision or visual\$ or sight\$)).tw.

39. (retinopath\$ adj3 (eye $\$$ or vision or visual\$ or sight $\$$ )).tw.

40. (DR adj3 (eye\$ or vision or visual\$ or sight\$)).tw.

41. or/34-40

42. $\exp$ Screening/

43. exp Vision Test/

44. Eye Examination/

45. Telemedicine/

46. Photography/

47. Eye Photography/

48. Ophthalmoscopy/

49. (ophthalmoscop\$ or fundoscop\$ or funduscop\$).ti.

50. ((exam\$ or photo $\$$ or imag\$) adj3 fundus).tw.

51. (photography or retinography).tw.

52. ((mydriatic or digital or retina\$ or fundus or steroscopic) adj3 camera).tw.

53. ((mydriatic or digital or retina $\$$ or fundus or steroscopic) adj3 imag\$).tw.

54. screen\$.tw.

55. ((eye\$ or retina\$ or ophthalm\$) adj4 exam\$).tw.

56. ((eye or vision or retinopathy or ophthalmic) adj4 test\$).tw.

57. ((eye\$ or retina\$ or ophthalm\$) adj4 visit\$).tw.

58. (telemedicine\$ or telemonitor\$ or telescreen\$ or telehealth or teleophthalmology).tw.

59. or/42-58

60. Health Care Quality/

61. Quality Improvement/

62. Health Care Delivery/

63. Integrated Health Care System/

64. service delivery.tw.

65. decision making.tw.

66. (consensus adj3 (process\$ or discuss)).tw.

67. stakeholder\$.tw.

68. Quality Control/

69. Total Quality Management/

70. quality assurance.tw.

71. (quality adj2 improv\$).tw.

72. total quality.tw.

73. continuous quality.tw.

74. quality management.tw.

Interventions to increase attendance for diabetic retinopathy screening (Review) 
75. (organisation\$ adj3 cultur\$).tw.

76. disease management/

77. program evaluation/

78. ((provider\$ or program\$) adj3 (monitor\$ or evaluate\$ or modif\$ or practice)).tw.

79. (implement $\$$ adj3 (improve $\$$ or change $\$$ or effort\$ or issue $\$$ or impede $\$$ or glossary or tool\$ or innovation $\$$ or outcome $\$$ or driv $\$$ or examin $\$$ or reexamin $\$$ or scale or strateg\$ or advis $\$$ or expert\$)).tw.

80. (need\$ adj3 assess\$).tw.

81. ((education\$ or learn\$) adj5 (continu\$ or material\$ or meeting or collaborat\$)).tw.

82. Medical audit/

83. (audit or feedback or compliance or adherence or training or innovation).ti.

84. (guideline\$ adj3 (clinical or practice or implement\$ or promot\$)).tw.

85. (outreach adj2 (service\$ or visit\$)).tw.

86. (intervention $\$$ adj3 (no or usual or routine or target\$ or tailor\$ or mediat\$)).tw.

87. usual care.tw.

88. reminder system/

89. remind\$.tw.

90. (improve adj3 (attend\$ or visit\$ or intervention\$ or adhere\$)).tw.

91. (increas $\$$ adj3 (attend\$ or visit $\$$ or intervention\$ or adhere\$)).tw.

92. (appointment $\$$ adj3 (miss\$ or fail\$ or remind\$ or follow up)).tw.

93. telephone/

94. telephone.tw.

95. Mobile Phone/

96. Mobile Application/

97. Teleconsultation/

98. (m-health or e-health or g-health or u-health).tw.

99. (phone\$ adj1 (smart or cell)).tw.

100. (smartphone\$ or cellphone\$).tw.

101. (hand adj1 held device\$).tw.

102. (mobile adj2 (health or healthcare or phone\$ or device\$ or monitor\$ or comput\$ or app or apps or application)).tw.

103. Internet/

104. Social Network/

105. (email\$ or text\$ or message\$).tw.

106. (letter or mail or mailed or print\$ or brochure $\$$ or newsletter\$).tw.

107. Primary Health Care/

108. General Practitioner/

109. Primary Prevention/

110. Preventive Health Service/

111. Community Care/

112. Community Health Nursing/

113. exp Transcultural Care/

114. Rural Health Care/

115. Ophthalmologist/

116. (Ophthalmologist $\$$ or Optometrist $\$$ or Optician\$ or Orthopist $\$$ or Refractionists).tw.

117. ((Ophthalmic or eye) adj3 (surgeon $\$$ or nurse $\$$ or technician $\$$ or officer $\$$ or assistant $\$$ or staff\$)).tw.

118. Clinical Practice/

119. Professional Practice/

120. Continuing Education/

121. (professional adj3 (practice or develop\$ or educat)).tw.

122. Nurse/

123. Nursing Discipline/

124. Nurse Attitude/

125. Nursing Education/

126. (nurse or nurses).tw.

127. pharmacist/

128. pharmacist\$.tw.

129. ((role or roles) adj3 expan\$).tw.

130. (task\$ adj3 shift\$).tw.

131. Electronic Medical Record/

132. Information System/

133. Data Base/

134. Computer System/

135. Hospital Information System/

Interventions to increase attendance for diabetic retinopathy screening (Review) 
136. ((health or healthcare) adj4 (record or management system\$)).tw.

137. (decision adj5 support).ti.

138. cost benefit analysis/

139. cost effectiveness analysis/

140. cost of illness/

141. cost control/

142. economic aspect/

143. financial management/

144. health care cost/

145. health care financing/

146. health economics/

147. hospital cost/

148. (fiscal or financial or finance or funding).tw.

149. cost minimization analysis/

150. (cost adj estimate\$).mp.

151. (cost adj variable\$).mp.

152. (unit adj cost\$).mp.

153. (economic\$ or pharmacoeconomic\$ or price $\$$ or pricing).tw.

154. exp Reimbursement/

155. (financial or economic or pay or payment or copayment or paid or fee or fees or monetary or money or cash or incentiv\$ or disincentiv \$).tw.

156. (insurance adj3 (health\$ or scheme\$)).tw.

157. or/60-156

158. exp Patient Attitude/

159. exp Health Behaviour/

160. (barrier\$ or obstacle\$ or facilitat\$ or enable\$).tw.

161. (uptake or takeup or attend\$ or accept\$ or adhere $\$$ or attitude $\$$ or participat $\$$ or facilitat $\$$ or utilisat $\$$ or utilizat $\$$ ).tw.

162. (complie or comply or compliance or noncompliance or non compliance\$).tw.

163. (encourag $\$$ or discourage $\$$ or reluctan $\$$ or nonrespon $\$$ or non respons $\$$ or refuse $\$$ ).tw.

164. (non-attend\$ or non attend\$ or dropout or drop out or apath\$).tw.

165. Health Education/

166. exp Patient Education/

167. Diabetes Education/

168. Help Seeking Behavior/

169. Patient Participation/

170. Patient Decision Making/

171. exp Health Promotion/

172. (health adj2 (promotion\$ or knowledge or belief\$)).tw.

173. (educat\$ adj2 (intervention $\$$ or information or material or leaflet)).tw.

174. exp Socioeconomics/

175. Income/

176. Social Class/

177. Social Status/

178. Educational Status/

179. ((school or education\$) adj3 (status or level\$ or attain\$ or achieve\$)).tw.

180. Employment/

181. Health Care Disparity/

182. Health Disparity/

183. Rural Population/

184. Rural Area/

185. Urban Population/

186. Urban Area/

187. exp Ethnic Group/

188. Ethnicity/

189. Race Difference/

190. Minority Groups/

191. Vulnerable Populations/

192. ((health\$ or social\$ or racial\$ or ethnic\$) adj5 (inequalit\$ or inequit\$ or disparit\$ or equit $\$$ or disadvantage $\$$ or depriv\$)).tw.

193. (disadvant $\$$ or marginali\$ or underserved or under served or impoverish $\$$ or minorit $\$$ or racial\$ or ethnic\$).tw.

194. or/158-193

195. 157 or 194

196. 33 and 41 and 59 and 195

Interventions to increase attendance for diabetic retinopathy screening (Review) 
197. (ranibizumab or bevacizumab or avastin or aflibercept or photocoagulation or coronary or cardiovascular).ti.

198. (blood glucose or blood pressure).ti.

199. (macula\$ adj2 (oedema or edema)).ti.

200. (cataract or intraocular or glaucoma).ti.

201. macula\$ degeneration.ti.

202. nerve fiber layer.ti.

203. or/197-202

204. 196 not 203

\section{Appendix 4. PsychINFO search strategy}

1. exp Treatment Effectiveness Evaluation/

2. exp Clinical Trials/

3. exp Placebo/

4. placebo\$.tw.

5. randomly.tw.

6. randomi\#ed.tw.

7. trial\$.tw.

8. ((singl\$ or doubl\$ or trebl\$ or tripl\$) adj3 (blind\$ or mask\$ or dummy)).tw.

9. (factorial\$ or allocat\$ or assign\$ or volunteer\$).tw.

10. (crossover $\$$ or cross over\$).tw.

11. (quasi adj (experimental or random\$)).tw.

12. (control\$ adj3 (trial\$ or study or studies or group\$)).tw.

13. or/1-12

14. diabetes/

15. ((diabet\$ or proliferative or non-proliferative) adj4 retinopath\$).tw.

16. (diabet\$ adj3 (eye\$ or vision or visual\$ or sight\$)).tw.

17. (retinopath\$ adj3 (eye\$ or vision or visual\$ or sight\$)).tw.

18. (DR adj3 (eye or vision or visual\$ or sight\$)).tw.

19. or/14-18

20. exp Screening/

21. ophthalmologic examination/

22. telemedicine/

23. (ophthalmoscop\$ or fundoscop\$ or funduscop\$).ti.

24. ((exam\$ or photo\$ or imag\$) adj3 fundus).tw.

25. (photography or retinography).tw.

26. ((mydriatic or digital or retina\$ or fundus or steroscopic) adj3 camera).tw.

27. ((mydriatic or digital or retina\$ or fundus or steroscopic) adj3 imag\$).tw.

28. screen\$.tw.

29. ((eye\$ or retina\$ or ophthalm\$) adj4 exam\$).tw.

30. ((eye or vision or retinopathy or ophthalmic) adj4 test\$).tw.

31. ((eye\$ or retina\$ or ophthalm\$) adj4 visit\$).tw.

32. (telemedicine\$ or telemonitor\$ or telescreen\$ or telehealth or teleophthalmology).tw.

33. or $/ 20-32$

34. 13 and 19 and 33

\section{Appendix 5. CPCI-S and ESCI search strategy}

\#11 \#10 AND \#2 AND \#1

\#10 \#9 OR \#8 OR \#7 OR \#6 OR \#5 OR \#4 OR \#3

\#9 TS = (photography OR retinography OR telemedicine* OR telemonitor ${ }^{\star}$ OR telescreen* OR telehealth OR teleophthalmology)

\#8 TS = (fundus NEAR/3 exam* OR fundus NEAR/3 photo* OR fundus NEAR/3 imag*)

\#7 TS = (imag ${ }^{\star}$ NEAR/3 mydriatic OR imag* NEAR/3 digital OR imag* NEAR/3 retina* OR imag ${ }^{\star}$ NEAR/3 fundus OR imag* NEAR/3 steroscopic OR camera NEAR/3 mydriatic OR camera NEAR/3 digital OR camera NEAR/3 retina* OR camera NEAR/3 fundus OR camera NEAR/3 steroscopic)

\#6 TI = (ophthalmoscop* OR fundoscop* OR funduscop*)

\#5 TS = (visit NEAR/4 eye* OR visit NEAR/4 retina* OR visit NEAR/4 ophthalmic)

\#4 TS $=\left(\right.$ exam ${ }^{\star}$ NEAR/4 eye ${ }^{\star}$ OR exam ${ }^{\star}$ NEAR/4 retina* OR exam $^{\star}$ NEAR/4 ophthalmic $)$

\#3 TS $=$ (screen ${ }^{\star}$ OR test ${ }^{\star}$ NEAR/4 eye OR test ${ }^{\star}$ NEAR/4 vision OR test ${ }^{\star}$ NEAR/4 retinopathy OR test ${ }^{\star}$ NEAR/4 ophthalmic)

\#2 TS = (diabetic NEAR/3 retinopath* OR diabetic NEAR/3 eye* OR diabetic NEAR/3 vision OR diabetic NEAR/3 visual* OR diabetic NEAR/3

sight* OR diabetic NEAR/3 proliferative OR diabetic NEAR/3 "non proliferative")

\#1 TS $=$ (clinical trial ${ }^{\star}$ OR research design OR comparative stud ${ }^{\star}$ OR evaluation stud ${ }^{\star}$ OR controlled trial ${ }^{\star}$ OR follow-up stud ${ }^{\star}$ OR prospective stud $^{\star}$ OR random* OR placebo* OR single blind* OR double blind ${ }^{\star}$ ) 


\section{Appendix 6. ProQuest Family Health search strategy}

ab(diabetic AND (retinopathy OR eye OR vision OR visual OR sight)) AND ab(screen OR screening OR test OR exam OR examination OR telemedicine ) AND ab(random OR randomly OR randomised OR randomized)

\section{Appendix 7. OpenGrey search strategy}

(screen OR test OR exam OR Ophthalmoscopy OR digital OR imaging OR fundus OR telemedicine OR telemonitor OR telescreen OR telehealth) AND diabetic retinopathy

\section{Appendix 8. ISRCTN search strategy}

(screen OR test OR exam OR ophthalmoscopy OR digital OR imaging OR fundus OR telemedicine OR telemonitor OR telescreen OR telehealth) within Condition: diabetic retinopathy

\section{Appendix 9. ClinicalTrials.gov search strategy}

(screen OR test OR exam OR Ophthalmoscopy OR digital OR imaging OR fundus OR telemedicine OR telemonitor OR telescreen OR telehealth) | Interventional Studies | diabetic retinopathy

\section{Appendix 10. WHO ICTRP search strategy}

Condition = diabetic retinopathy AND Intervention = screen OR test OR exam OR Ophthalmoscopy OR digital OR imaging OR fundus OR telemedicine OR telemonitor OR telescreen OR telehealth

\section{CONTRIBUTIONS OFAUTHORS}

Protocol

All author were involved in the development of the protocol for this review.

Review

$\mathrm{JGL}$ and JB screened titles and abstracts.

JGL and EGR extracted data and performed 'risk of bias assessments'.

EGR, FL and JF performed BCT coding.

SR designed and developed the algorithm for resource requirement.

PA conducted the economic evaluation review (with input from LV).

JGL inputted data into Revman. CB checked the data.

CB conducted the statistical analysis (checked by JGL).

$J G L$ produced the first draft of the review and all authors reviewed and commented on the draft.

\section{DECLARATIONS OF INTEREST}

JGL: None known

EG-R: None known

FL: None known

JP: None known

JB: None known

$\mathrm{NI}$ : None known

PA: None known

CB: None known

JF: None known

JG: None known

TP: None known

SR: None known

LV: None known

\section{SOURCES OF SUPPORT}

\section{Internal sources}

- National Institute for Health Research Health Technology Assessment (NIHR-HTA), UK.

This review has been carried out as part of an evidence synthesis project funded by NIHR-HTA (Project reference Number 13/137/05). 


\section{External sources}

- National Institute for Health Research (NIHR), UK.

* This review is funded by the NIHR health technology assessment programme.

* Richard Wormald, Co-ordinating Editor for the Cochrane Eyes and Vision (CEV) acknowledges financial support for his CEV research sessions from the Department of Health through the award made by the National Institute for Health Research to Moorfields Eye Hospital NHS Foundation Trust and UCL Institute of Ophthalmology for a Specialist Biomedical Research Centre for Ophthalmology.

* This review was supported by the National Institute for Health Research, via Cochrane Infrastructure funding to the CEV UK editorial base.

The views expressed in this publication are those of the authors and not necessarily those of the NIHR, NHS, or the Department of Health.

\section{DIFFERENCES BETWEEN PROTOCOLANDREVIEW}

In addition to the prespecified covariates for metaregression we also investigated the effect of study design (individual versus cluster-RCT) and risk of bias (high versus low). We had originally planned to conduct a sensitivity analysis to compare studies of high versus low risk of bias.

Only nine of the 30 included cluster-trials reported an ICC. The most commonly-reported value was imputed for studies with no estimates of ICCS. We therefore conducted an unplanned sensitivity analysis to investigate the impact on the pooled effect estimate of using the lower and upper range values.

The checklists used for the economic analysis differed from those that were originally stated in our published Cochrane protocol, due to the recent updates of the methods for the incorporation of economic evidence into Cochrane Intervention Reviews. See Table 4 and Table 5 for the completed CHEERS and CHEC checklists for each included economic evaluation.

\section{N DEX TERMS}

\section{Medical Subject Headings (MeSH)}

${ }^{\star}$ Quality Improvement [economics]; Costs and Cost Analysis; Diabetes Mellitus, Type 1 [ ${ }^{*}$ complications]; Diabetes Mellitus, Type 2 [ ${ }^{*}$ complications]; Diabetic Retinopathy [ ${ }^{*}$ diagnosis]; Patient Compliance [psychology] [ ${ }^{*}$ statistics \& numerical data]; Randomized Controlled Trials as Topic

\section{MeSH check words}

Humans 